\title{
HYDROLOGIC AND SEDIMENTOLOGIC DATA
}

COLLECTED DURING THREE CRUISES ON

THE MISSISSIPPI RIVER AND SOME OF

ITS TRIBUTARIES FROM MINNEAPOLIS,

MINNESOTA, TO NEW ORLEANS,

LOUISIANA, JULY 1991-MAY 1992

By JOHN A. MOODY and ROBERT H. MEADE

U.S. GEOLOGICAL SURVEY

Open-File Report $94-474$ 


\section{U.S. DEPARTMENT OF THE INTERIOR \\ BRUCE BABBITT, Secretary \\ U.S. GEOLOGICAL SURVEY \\ Gordon P. Eaton, Director}

The use of trade, product, industry, or firm names is for descriptive purposes only and does not imply endorsement by the U.S. Government.

For additional information write to:

Chief, Branch of Regional Research U.S. Geological Survey

Box 25046, MS 418

Denver Federal Center

Denver, CO 80225
Copies of this report can be purchased from:

U.S. Geological Survey

Earth Science Information Center

Open-File Reports Section

Box 25286, MS 517

Denver Federal Center

Denver, CO 80225 


\section{CONTENTS}

Page

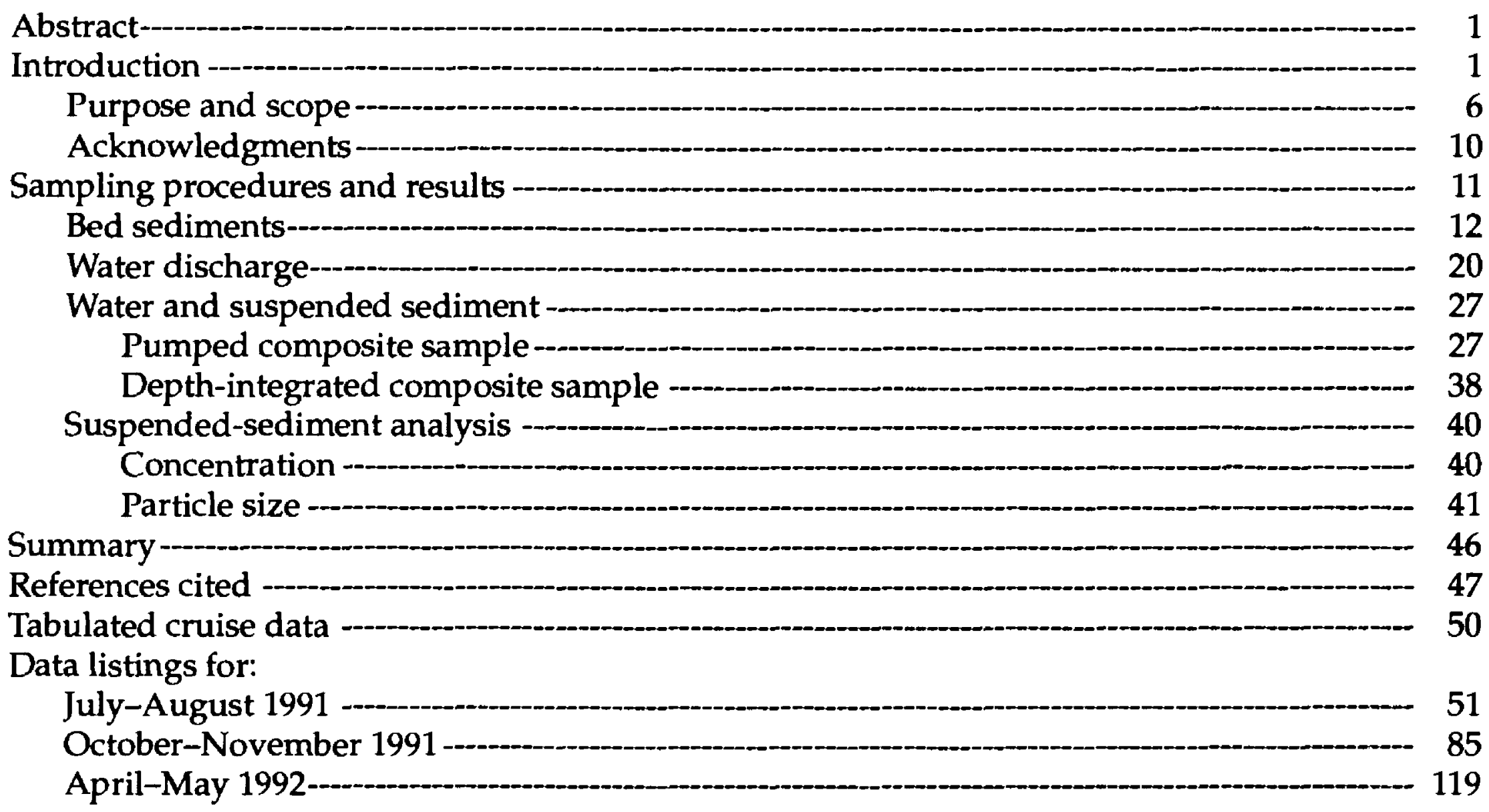

FIGURES

Figure 1. Map showing Mississippi River and some of its tributaries-a- 2

2. Graph showing mean annual discharge of the Mississippi River -

3. Graph showing water discharge at selected locations upstream from

St. Louis, Missouri on the Upper Mississippi River and some of its tributaries from June 1991 through May 1992 --ons downstream from

4. Graph showing water discharge at selected locations downstream from
St. Louis, Missouri on the Upper Mississippi River and some of its tributaries from June 1991 through May 1992 - 9

5. Diagram showing suspended-sediment sampler with current meter-_-_-_-_._- 21

6. Flow diagram for processing the suspended-sediment sample collected by the depth-integration method 
7-22. Graphs showing depth, mean velocity, and specific conductance of surface wate* versus the distance from the left edge of water for the July-August 1991 cruise:

7. Mississippi River above St. Anthony Falls, Minn.

8. Minnesota River at Mile 3.5, Minn.

9. Mississippi River at Hastings, Minn.

10. Mississippi River near Pepin, Wis.

11. Mississippi River at Trempealeau, Wis.

12. Mississippi River below Lock and Dam 9, Wis.

13. Mississippi River at Clinton, Iowa

14. Mississippi River at Keokuk, Iowa

15. Mississippi River near Winfield, Mo.

16. Illinois River at Hardin, Ill.

17. Missouri River at St. Charles, Mo.

18. Mississippi River at Thebes, Ill.-- 75

19. Ohio River at Olmsted, Ill. - 77

20. Mississippi River below Vicksburg, Miss. - 79

21. Mississippi River near St. Francisville, La.-- 81

22. Mississippi River below Belle Chasse, La. - - 83

23-38. Graphs showing depth, mean velocity, and specific conductance of surface water versus the distance from the left edge of water for the OctoberNovember 1991 cruise:

23. Mississippi River above St. Anthony Falls, Minn. - - 87

24. Minnesota River at Mile 3.5, Minn. - 89

25. Mississippi River at Hastings, Minn. $-\cdots$

26. Mississippi River near Pepin, Wis. - 93

27. Mississippi River at Trempealeau, Wis.--_- 95

28. Mississippi River below Lock and Dam 9, Wis. -- 97

29. Mississippi River at Clinton, Iowa - 99

30. Mississippi River at Keokuk, lowa - 101

31. Mississippi River near Winfield, Mo. -

32. Illinois River at Hardin, Ill. - 105

33. Missouri River at St. Charles, Mo. - 107

34. Mississippi River at Thebes, Ill.--109

35. Ohio River at Olmsted, Ill. - 111

36. Mississippi River below Vicksburg, Miss. - 113

37. Mississippi River near St. Francisville, La.--.- 115

38. Mississippi River below Belle Chasse, La. - $-1 .--117$

39-58. Graphs showing depth, mean velocity, and specific conductance of surface water versus the distance from the left edge of water for the April-May 1992 cruise:

39. Mississippi River above St. Anthony Falls, Minn. - 121

40. Mississippi River at Mile 844.0, Minn. - 123

41. Minnesota River at Mile 3.5, Minn. - 125

42. Minnesota River at Mile 0.0, Minn. - 127

43. Mississippi River at Hastings, Minn.-129

44. St. Croix River at Mile 0.0, Minn.-Wis. 131 
Figures 39-58.--Continued

45. Mississippi River near Pepin, Wis. 133

46. Chippewa River at Mile 2.0, Wis. - 135

47. Mississippi River at Trempealeau, Wis. 137

48. Mississippi River below Lock and Dam 9, Wis. - 139

49. Mississippi River at Clinton, Iowa -- 141

50. Mississippi River at Keokuk, Iowa - 143

51. Mississippi River near Winfield, Mo. - 145

52. Illinois River at Hardin, Ill. - 147

53. Missouri River at St. Charles, Mo. - 149

54. Mississippi River at Thebes, Ill.-- 151

55. Ohio River at Olmsted, Ill. - 153

56. Mississippi River below Vicksburg, Miss. - 155

57. Mississippi River near St. Francisville, La.-157

58. Mississippi River below Belle Chasse, La. … 159

TABLES

Table 1. Drainage area and mean annual discharge for some of the tributaries to the Mississippi River--_-_- 4

2. Sampling sites for July-August 1991 cruise, October-November 1991 cruise, and April-May 1992 cruise---

3-5.Particle size of bed material collected from the Mississippi River and some of its tributaries for:

3. July-August 1991 cruise

4. October-November 1991 cruise -

5. April-May 1992 cruise -_-_- 18

6-8. Summary of the hydrologic data of the Mississippi River and some of its tributaries for:

6. July-August 1991 cruise-- 22

7. October-November 1991 cruise - 23

8. April-May 1992 cruise---_or 24

9. Summary of water discharge for the Mississippi River and some of its tributarie: for three cruises

10-12. Volumes of river water collected and processed, associated sampling parameters. and percent recovery of suspended sediment for:

10. July-August 1991 cruise -

11. October-November 1991 cruise -.......- 30

12. April-May 1992 cruise --.-_ 32

13-15. Concentration of suspended sediment in composite samples collected from the Mississippi River and some of its tributaries and the corresponding sediment discharges for:

13. July-August 1991 cruise 35

14. October-November 1991 cruise-- 36

15. April-May 1992 cruise - 37

16-18. Particle-size distribution of suspended-sediment samples collected from the Mississippi River and some of its tributaries for:

16. July-August 1991 cruise --- 43

17. October-November 1991 cruise -

18. April-May 1992 cruise - 


\section{CONVERSION FACTORS}

\section{Multiply}

micrometer $(\mu \mathrm{m})$

millimeter $(\mathrm{mm})$

centimeter $(\mathrm{cm})$

meter $(\mathrm{m})$

kilometer $(\mathrm{km})$

square meter $\left(\mathrm{m}^{2}\right)$

square kilometer $\left(\mathrm{km}^{2}\right)$

milliliter $(\mathrm{mL})$

liter (L)

cubic meter $\left(\mathrm{m}^{3}\right)$

centimeter per second $(\mathrm{cm} / \mathrm{s})$

meter per second $(\mathrm{m} / \mathrm{s})$

kilometer per hour $(\mathrm{km} / \mathrm{h})$

liter per minute $(\mathrm{L} / \mathrm{min})$

cubic meter per second $\left(\mathrm{m}^{3} / \mathrm{s}\right)$

cubic meter per year $\left(\mathrm{m}^{3} / \mathrm{yr}\right)$

cubic kilometer per year $\left(\mathrm{km}^{3} / \mathrm{yr}\right)$

metric ton per day (metric ton/d)

milligram (mg)

gram $(\mathrm{g})$

metric ton

degree Celsius $\left({ }^{\circ} \mathrm{C}\right)$
By

To obtain

\section{Length}

0.00003937

0.03937

0.3937

3.281

0.6214

Area

10.76

0.3861

Volume

0.03382

0.2642

35.31

Velocity

0.03281

3.281

0.6214

\section{Discharge}

0.2642

35.31

$35.31 \times 10^{9}$

1.102

Mass

0.00003527

0.002205

2,205

Temperature

$\mathrm{F}=1.8 \mathrm{x}^{\circ} \mathrm{C}+32$ inch

inch

inch

foot

mile

square foot

square mile

ounces, fluid gallon

cubic foot

foot per second foot per second mile per hour

gallon per minute cubic foot per second cubic foot per year cubic foot per year short ton per a day

ounce, avoirc'upois pound, avoirdupois pound, avoirdupois

degree Fahreרheit 


\title{
HYDROLOGIC AND SEDIMENTOLOGIC DATA COLLECTED DURING THREE CRUISES ON THE MISSISSIPPI RIVER AND SOME OF ITS TRIBUTARIIES FROM MINNEAPOLIS, MINNESOTA, TO NEW ORLEANS, LOUISIANA, JULY 1991-MAY 1992
}

\author{
By John A. Moody and Robert H. Meade
}

\begin{abstract}
Bed sediments, water, and suspended-sediment samples were collected and water dis tharge was measured at 12 sites on the Mississippi River. These sites and 14 sites on tributaries to the Mississippi River were sampled on three cruises during an 11-month period from July 5, 1991, to May 10, 1992. Discharge-weighted pumping and depth-integration methods were used at several equally spaced locations across the river to collect composite samples of river water containing suspended sediment. Water discharge was measured by the depth-integration methot and ranged from about 220 cubic meters per second for Mississippi River above St. Anthony Falls, Minnesota, in October 1991 to 21,700 cubic meters per second below Vicksburg, Mississi วpi, in May 1992. Concentration of suspended sediment in the Mississippi River varied from $c$ milligrams per liter downstream from Lake Pepin to 701 milligrams per liter at Thebes, Illinois.

This report contains the following hydrologic data associated with the samples: crosssectional area of the river, water depths, depth-averaged velocities, water discharge, and temperature, $\mathrm{pH}$, and specific conductance of the surface water. It contains the following sedimentologic data: concentrations of the suspended sand fraction, silt and clay fraction, and colloid fraction, and particle sizes of bed sediment and suspended sediment. These data provide the framowork for interpreting subsequent chemical analyses of the water and suspended-sediment samples collected during the three cruises and for calculating bed-load transport in the Mississipp: River and some of its tributaries.
\end{abstract}

\section{INTRODUCTION}

The Mississippi River drains about 40 percent of the conterminous United States and commonly is divided into two parts-the Lower Mississippi River and the Upper Miss'ssippi River. Distances on the Lower Mississippi River begin at mile 0.0 where the mouth of the river divides into three separate channels at Head of Passes in Louisiana and increase upstream to the mouth of the Ohio River at Cairo, Illinois (Lower Mississippi River Mile 953.8). Distances on the Upper Mississippi River begin at mile 0.0 at the mouth of the Ohio River and increase upstream to the source of the Mississippi River in Minnesota (fig. 1). The Lower and Upper Miss'ssippi Rivers are very different in their hydrologic character. The Lower Mississippi River is a freeflowing river, whereas about 80 percent of the navigable length of the Upper Mississippi Kiver is controlled by a series of 29 navigation locks and dams creating a stair-step series of navigation pools. The mean annual water discharge of the Mississippi River increases from about $7 \mathrm{~km}^{3} / \mathrm{yr}$ near Minneapolis, Minnesota (period of record: 1931-88; U.S. Geological Survey, Minnesota, 1988), to about $500 \mathrm{~km}^{3} / \mathrm{yr}$ at Vicksburg, Mississippi (period of record: 1931-91), while the corre- 


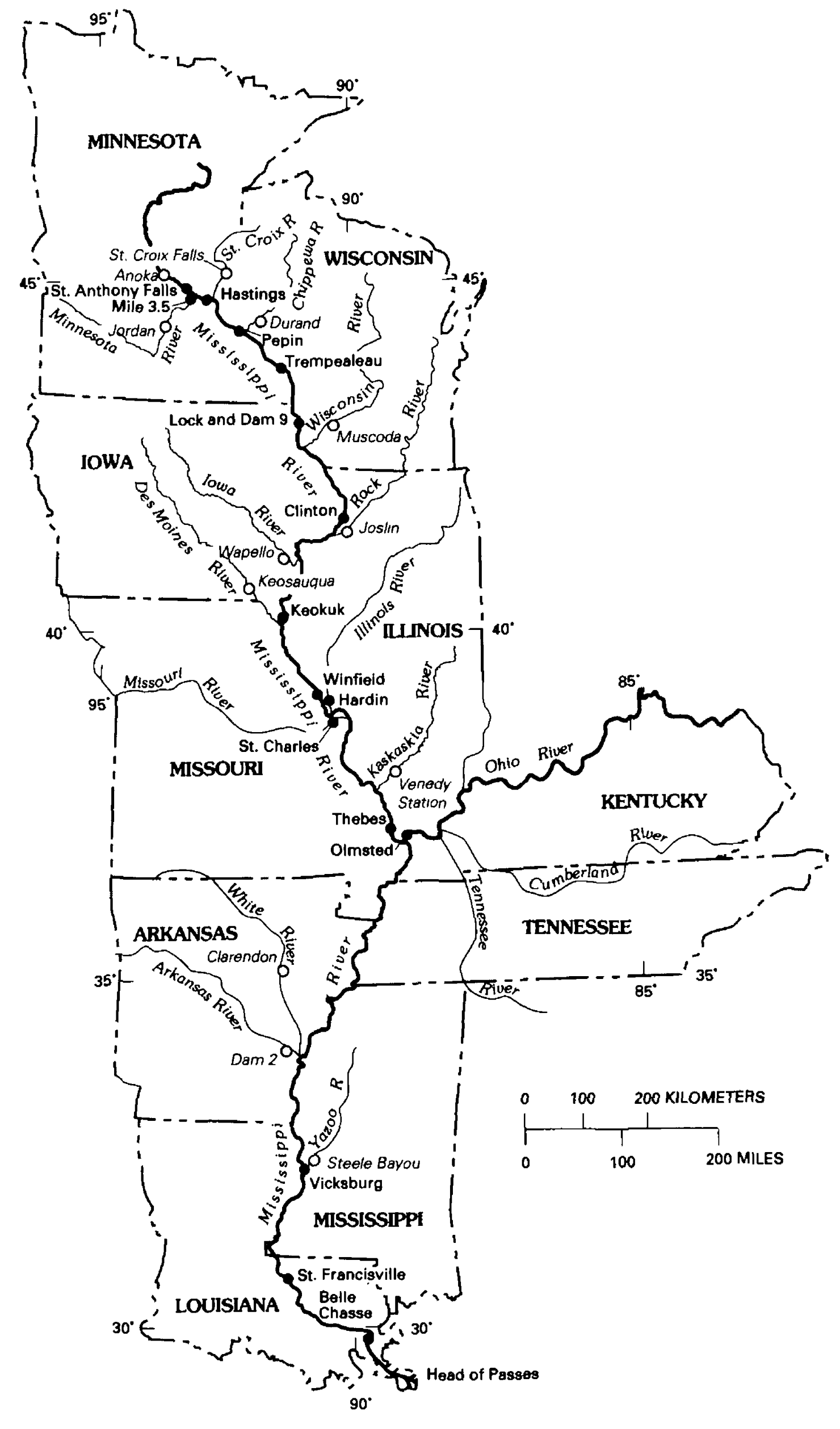

GULF OF MEXICO

Figure 1.--Mississippi River and some of its tributaries. Sampling sites are shown as solid circles. Other geographical sites are shown as open circles. 
sponding sediment discharge increases from about $0.2 \times 10^{6}$ metric tons/yr (Keown and others, 1981) to about $200 \times 10^{6}$ metric tons/yr (Moody and Meade, 1992). The mean annual water discharge of the Mississippi River more than doubles with the addition of water from the Minnesota and St. Croix Rivers just downstream from Minneapolis, Minnesota (see table 1). The mean annual discharge of the river increases slowly with each tributary input (table 1) until the combined water discharges of the Illinois and Missouri Rivers more than double the discharge of the Upper Mississippi River to about $16 \mathrm{C} \mathrm{km}^{3} /$ yr (fig. 2). The addition of water from the Ohio River again doubles the discharge of the Mississippi River, but the contributions of water by the White and Arkansas Rivers (each having discharges two to five times greater than the combined flow of the Minnesota and St. Croix Rivers) only account for about 5 to 8 percent of the discharge of the Lower Mississippi River at Vicksburg, Mississippi. At 191 k'lometers downstream from Vicksburg, approximately 25 percent of the water and sediment discharges are diverted from the Mississippi River by the Old River Control Structures into the Atchafalaya River and then into the Gulf of Mexico. The remaining water $\left(375 \mathrm{~km}^{3} / \mathrm{yr}\right)$ and sediment $\left(150 \times 10^{6}\right.$ metric tons / yr) are discharged by the Mississippi River directly into the Gulf of Mexico (Moody and Meade, 1992).

The U.S. Geological Survey began a study of the sediment-transported pollutants in the Mississippi River and some of its tributaries in 1987 and made three research cruises at low water in July-A ugust 1987, in November-December 1987, and during the record low water in May-June 1988 (Mood'y and Meade, 1992). Four cruises were made during high waters in March-April 1989, in June 19.99, in February-March 1990, and in May-June 1990 (Moody and Meade, 1993). These cruises started about $50 \mathrm{~km}$ upstream from the confluences of the Upper Mississippi-Missouri-Illinois Rivers near St. Louis, Missouri, and ended about $40 \mathrm{~km}$ downstream from New Orleans, Louisiana.

The broad objectives of this multidisciplinary study were to investigate the movement, mixin $\sim$, and storage processes of sediment-associated and dissolved pollutants in the Mississippi River sy'stem. Some specific objectives were to:

1. Examine the geochemistry of the suspended silt, clay, and colloidal material, and disrolved phases of river water;

2. Assess the contamination of bed sediments stored in the navigation pools;

3. Understand the compartmentalization of organic and inorganic chemicals among the water, sediment, and biotic phases;

4. Investigate the mixing, partitioning, and redistribution processes of the various pollutants downstream from major river confluences;

5. Understand the movement, storage, and remobilization of suspended sediment and asscciated pollutants at seasonal or longer time periods; and

6. Predict the location and travel time of water masses and the associated sediment and pollutants. 


\section{Table 1.--Drainage area and mean annual discharge for some of the tributaries to the Misslssippi Rlver}

[Mean annual discharge is given for the gaging station closest to the mouth of the tributary; $\mathrm{km}^{2}$, square kilometers; $\mathrm{km}^{3} / \mathrm{yr}$; cubic kilometer per year; $\%$, percent]

\begin{tabular}{|c|c|c|c|c|}
\hline Tributary & $\begin{array}{l}\text { Length } \\
\text { of } \\
\text { record } \\
\text { (years) }\end{array}$ & $\begin{array}{c}\text { Drainege } \\
\text { area } \\
\left(\mathbf{k m}^{2}\right)\end{array}$ & $\begin{array}{c}\text { Mean } \\
\text { annual } \\
\text { discharge } \\
\left(\mathbf{k m}^{\mathbf{3}} / \mathbf{y r}\right)\end{array}$ & $\begin{array}{l}\text { Percent of } \\
\text { discharge of } \\
\text { Missis -iopi River } \\
\text { downs'ream from } \\
\text { the mouth of the } \\
\text { tritystary } \\
(\%)\end{array}$ \\
\hline Minnesota R. near Jordan, Minn. ${ }^{1}$ & 54 & 42,000 & 3.4 & 34 \\
\hline St. Croix R. at St. Croix Falls, Wis. ${ }^{1}$ & 86 & 16.200 & 3.9 & 25 \\
\hline Chippewa R. at Durand, Wis. ${ }^{2}$ & 61 & 23,300 & 6.8 & 27 \\
\hline Wisconsin R. at Muscoda, Wis. ${ }^{2}$ & 76 & 26,900 & 7.8 & 20 \\
\hline Rock R. near Joslin, $111 .^{3}$ & 51 & 24,700 & 5.5 & 11 \\
\hline lowa R. at Wapello, lowa ${ }^{4}$ & 75 & 32,400 & 6.2 & 11 \\
\hline Des Moines R. at Keosauqua, lowa ${ }^{4}$ & 80 & 36.400 & 5.2 & 8 \\
\hline 1llinois R. at Meredosia, $111 .^{5}$ & 51 & 67,400 & 19.6 & 23 \\
\hline Missouri R. at Hermann, Mo. ${ }^{6}$ & 88 & $1,358,000$ & 72.0 & 44 \\
\hline Kaskaskia R. near Venedy Station, $\left[11 .^{3}\right.$ & 21 & 11,400 & 3.3 & 2 \\
\hline Ohio R. at Metropolis, [11. ${ }^{6}$ & 58 & 528,300 & 243.1 & 57 \\
\hline White R. at Devalls Bluff, Ark. ${ }^{7}$ & 22 & 60,700 & 23.3 & 5 \\
\hline Arkansas R. at Murray Dam, Ark. ${ }^{?}$ & 62 & 409,300 & 37.4 & 8 \\
\hline \multirow[t]{2}{*}{ Yazoo R. at Redwood. Miss. ${ }^{6}$} & 20 & 35,800 & 12.3 & 3 \\
\hline & & & 449.8 & \\
\hline
\end{tabular}

'U.S. Geological Survey. Water Resources Data for Minnesota, Water Year 1988, volume 2.

${ }^{2}$ U.S. Geological Survey, Water Resources Data for Wisconsin, Water Year 1989.

${ }^{3}$ U.S. Geological Survey, Water Resources Data for Illinois, Water Year 1989, volume I.

${ }^{4}$ U.S. Geological Survey, Water Resources Data for lowa, Water Year 1989.

${ }^{5}$ U.S. Geological Survey, Water Resources Data for Illinois, Water Year 1989, volume 2.

${ }^{6}$ Moody and Meade, 1992.

${ }^{7}$ U.S. Geological Survey, Water Resources Data for Arkansas, Water Year 1989. 


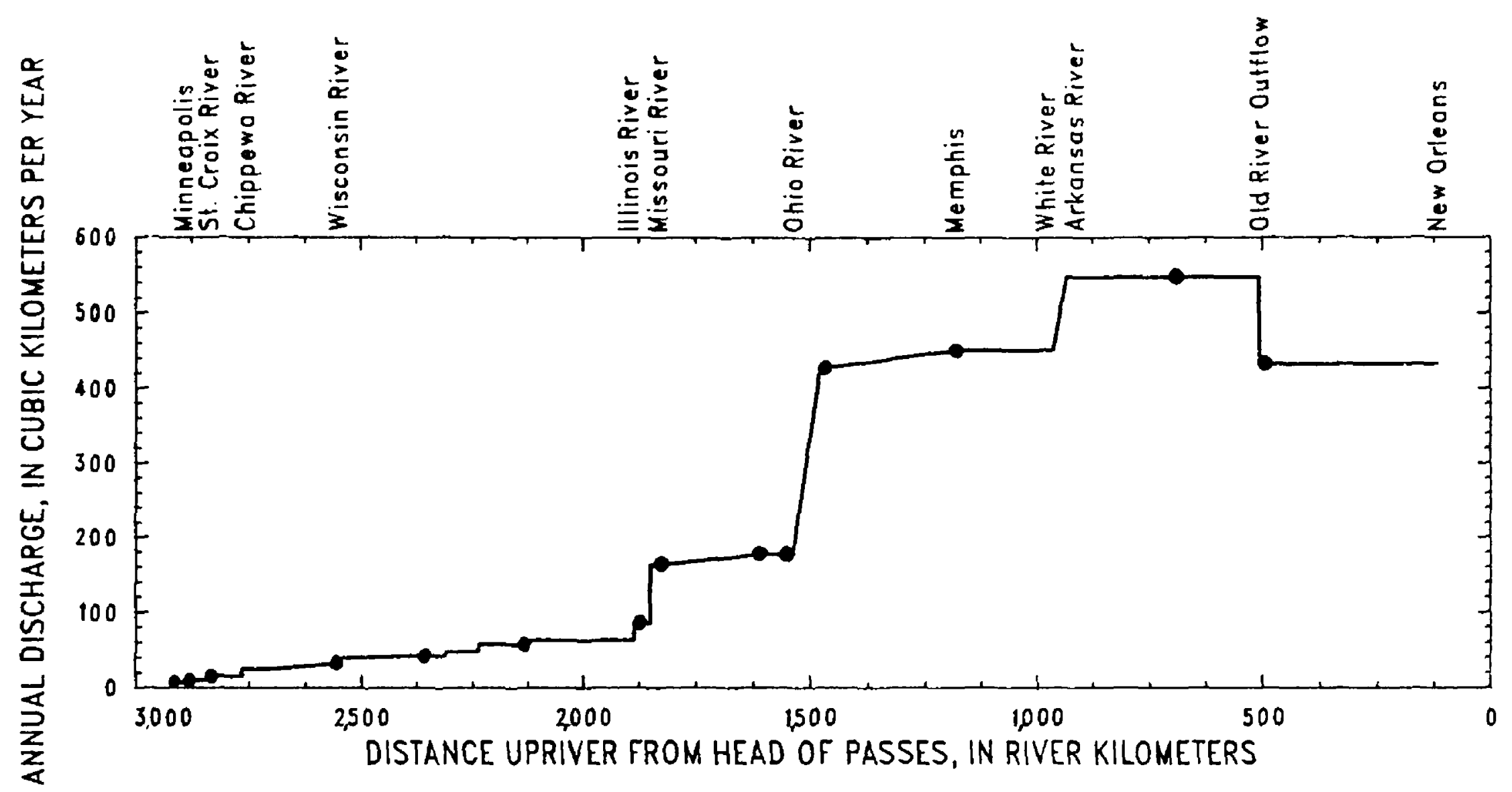

Figure 2. Mean annual discharge of the Mississippi River for period of record prior to 1989. Solid circles are discharge at gaging stations. (Data from U.S. Geological Survey publications: Water-Resources Data for Minnesota, for lowa, and for Missouri; and from Don Flowers, U.S. Army Corps of Engineers, oral commun., 1992). 


\section{Purpose and Scope}

The purpose of this report is (1) to describe the sampling procedures used to collect and process bedsediment samples and a representative water and suspended-sediment sample for physical and chemical analysis, and (2) to publish the hydrologic and sedimentologic data collected durin $:$ three cruises. The hydrologic data are river width, cross-sectional area of the river, water depths, dopth-averaged velocities, and water discharge. The sedimentologic data are concentrations of suspende $t$ sand (greater than $63 \mu \mathrm{m}$ ) and silt and clay (finer than $63 \mu \mathrm{m}$ ) and particle-size distributions of the bed sediment and suspended sediment. Temperature, $\mathrm{pH}$, and specific conductance of the surface water are listed in this report, but inorganic and organic chemical data are published elsewhere. The hydrologic data that are published in this report provide some of the necessary framework for interpreting the chemical data.

This report includes data from three cruises that collected samples from sites on the Mississippi River starting at Minneapolis, Minnesota, and ending about $40 \mathrm{~km}$ downstream from New Orleans, Louisiana (fig. 1). Samples also were collected from sites within $40 \mathrm{~km}$ of the mouths of the following tributaries: Minnesota, St. Croix, Chippewa, Wisconsin, Rock, Iowa, Des Moines, Illinois, Missouri, Kaskaskia, Ohio, White, Arkansas, and Yazoo Rivers. The sampling-site locations for each cruise are listed in table 2. The July-August 1991 cruise (July 5 through August 7, 1991) and the OctoberNovember 1991 cruise (October 7 through November 13, 1991) were during periods of relatively lowwater discharge, but the April-May 1992 cruise (April 6 through May 10, 1992) was during a period of relatively high discharge on the Mississippi River (figs. 3 and 4). 


\section{Table 2.--Sampling sites for July-August 1991 cruise, October-November 1991 cruise, and Aprił May 1992 crulse}

[River miles taken from navigation charts, U.S. Army Corps of Engineers, 1983?, 1986?, 1987a, $1987 b, 1989 a ?, 1989 b$ ?, the $x$ designates that the site was sampled]

\begin{tabular}{|c|c|c|c|c|}
\hline Sampling slte & $\begin{array}{l}\text { River } \\
\text { mile }^{t}\end{array}$ & $\begin{array}{l}\text { July } \\
\text { August } \\
1991\end{array}$ & $\begin{array}{c}\text { October- } \\
\text { November } \\
1991\end{array}$ & $\begin{array}{l}\text { Afrit } \\
\text { May } \\
1 \text { Ma? }\end{array}$ \\
\hline Mississippi R. above St. Anthony Falls, Minn. & UM 857.7 & $x^{2}$ & $x$ & $\mathrm{x}$ \\
\hline Minnesota R. at Mile 3.5, Minn. & MN 3.5 & $\mathbf{x}$ & $\mathrm{x}$ & $\mathrm{x}$ \\
\hline Mississippi R. at Hastings, Minn. & UM 812.2 & $\mathbf{x}$ & $\mathrm{x}$ & $\mathrm{x}$ \\
\hline St. Croix R. at Mile 0.5 , Wis. & SC 0.5 & $\mathrm{x}$ & $\mathbf{x}$ & $\mathrm{x}$ \\
\hline Mississippi R. near Pepin, Wis. & UM 764.5 & $\mathrm{x}$ & $\mathrm{x}$ & $\mathrm{x}$ \\
\hline Chippewa R. at Mile 1.7 , Wis. & $\mathrm{CH} 1.7$ & $\mathbf{x}$ & $\mathbf{x}$ & $\mathbf{x}$ \\
\hline Mississippi R. at Trempealeau, Wis. & UM 713.8 & $\mathbf{x}$ & $\mathbf{x}$ & $\mathbf{x}$ \\
\hline Mississippi R. below Lock and Dam 9, Wis. & UM 639.7 & $\mathbf{x}$ & $\mathrm{x}$ & $\mathbf{x}$ \\
\hline Wisconsin R. at Mile $\sim 1.0$, Wis. & WI 1.0 & $x$ & $\mathrm{x}$ & $x$ \\
\hline Mississippi R. at Clinton, lowa & UM 520.3 & $\mathrm{x}$ & $\mathrm{x}$ & $\mathbf{x}$ \\
\hline Rock R. at Mile $\sim 1.0$, Ill. & RK 1.0 & $x$ & $\mathrm{x}$ & $\mathbf{x}$ \\
\hline Iowa $R$, at Mile $\sim 1.0$, lowa & IA 1.0 & $\mathbf{x}$ & $x$ & $x$ \\
\hline Mississippi R. at Keokuk, lowa & UM 363.1 & $\mathbf{x}$ & $\mathbf{x}$ & $\mathbf{x}$ \\
\hline Des Moines R. at Mile $\sim 1.0$, lowa & DM 1.0 & $\mathrm{x}$ & $\mathrm{x}$ & $x$ \\
\hline Mississippi R. near Winfield, Mo. & UM 239.2 & $\mathbf{x}$ & $\mathrm{x}$ & $\mathbf{x}$ \\
\hline Illinois R. at Hardin, Ill. & IL 21.8 & $x$ & $\mathrm{x}$ & $x$ \\
\hline Missouri R. at St. Charles, Mo. & MO 24.8 & $\mathrm{x}$ & $x$ & $x^{3}$ \\
\hline Kaskaskia R. at Mile 1.5, Ill. & KA 1.5 & $\mathrm{x}$ & $x$ & $x$ \\
\hline Mississippi R. at Thebes, Ill. & UM 44.0 & $\mathrm{x}$ & $x$ & $x$ \\
\hline Ohio R. at Olmsted, IIl. & OH 965.0 & $x^{4}$ & $x$ & $\mathbf{x}$ \\
\hline White R. at Mile 1.2, Ark. & WH 1.2 & $\mathbf{x}$ & $x$ & $x$ \\
\hline Arkansas R. at Mile 0.0 , Ark. & AR 0.0 & $\mathrm{x}$ & $\mathbf{x}$ & $x$ \\
\hline Yazoo R. at Mile $\sim 3.0$, Miss. & $\mathrm{YZ} \sim 3.0$ & $\mathbf{x}$ & $\mathbf{x}$ & $x^{5}$ \\
\hline Mississippi R. below Vicksburg. Miss. & LM 433.4 & $\mathrm{x}$ & $\mathrm{x}$ & $x$ \\
\hline Mississippi R. near St. Francisville, La. & LM 266.4 & $\mathbf{x}$ & $\mathbf{x}$ & $\mathbf{x}$ \\
\hline Mississippi R. below Belle Chasse, La. & LM 73.1 & $x^{6}$ & $x$ & $\mathbf{x}$ \\
\hline
\end{tabular}

'UM, Upper Mississippi River miles measured upriver from confluence with Ohio River. LM, Lower Mississippi River miles measured upriver from Head of Passes, Louisiana MN, Minnesota River miles measured upriver from confluence with the Upper Mississippi River (UM 844.0). SC, St. Croix River miles measured upriver from confluence with the Upper Mississippi River (UM 8II.3).

$\mathrm{CH}$, Chippewa River miles measured upriver from confluence with the Upper Mississippi River (UM 763.5). WI, Wisconsin River miles measured upriver from confluence with the Upper Mississippi River (UM 630.6).

RK, Rock River miles measured upriver from confluence with the Upper Mississippi River (UM 479.0). IA, lowa River measured upriver from confluence with the Upper Mississippi River (UM 434.0). DM, Des Moines River measured upriver from confluence with the Upper Mississippi River (UM 361.5). IL, Illinois River miles measured upriver from confluence with Upper Mississippi River (UM 21 8.0). MO, Missouri River miles measured upriver from confluence with Upper Mississippi River (UM 195.3). KA, Kaskaskia River measured upriver from confluence with the Upper Mississippi River (UM 117.3). $\mathrm{OH}$, Ohio River miles measured downriver from Pittsburgh, Pennsylvania. Ohio-Mississippi confluence is at Ohio River mile 981.5 and Lower Mississippi River mile 953.8.

WH, White River miles measured upriver from confluence with Lower Mississippi River (LM 598.8). AR, Arkansas River miles measured upriver from confluence with Lower Mississippi River (LM 581.5)

YZ, Yazoo River miles measured upriver from confluence with Lower Mississippi River (LM 437.2).

${ }^{2}$ Sample was collected at Upper Mississippi River mile 858.3.

${ }^{3}$ Sample was collected at Missouri River mile 29.4.

${ }^{4}$ Sample was collected at Ohio River mile 965.5 .

${ }^{5}$ Sample was collected at Yazoo River mile 9.0

${ }^{6}$ Sample was collected at Lower Mississippi River mile 72.8 . 

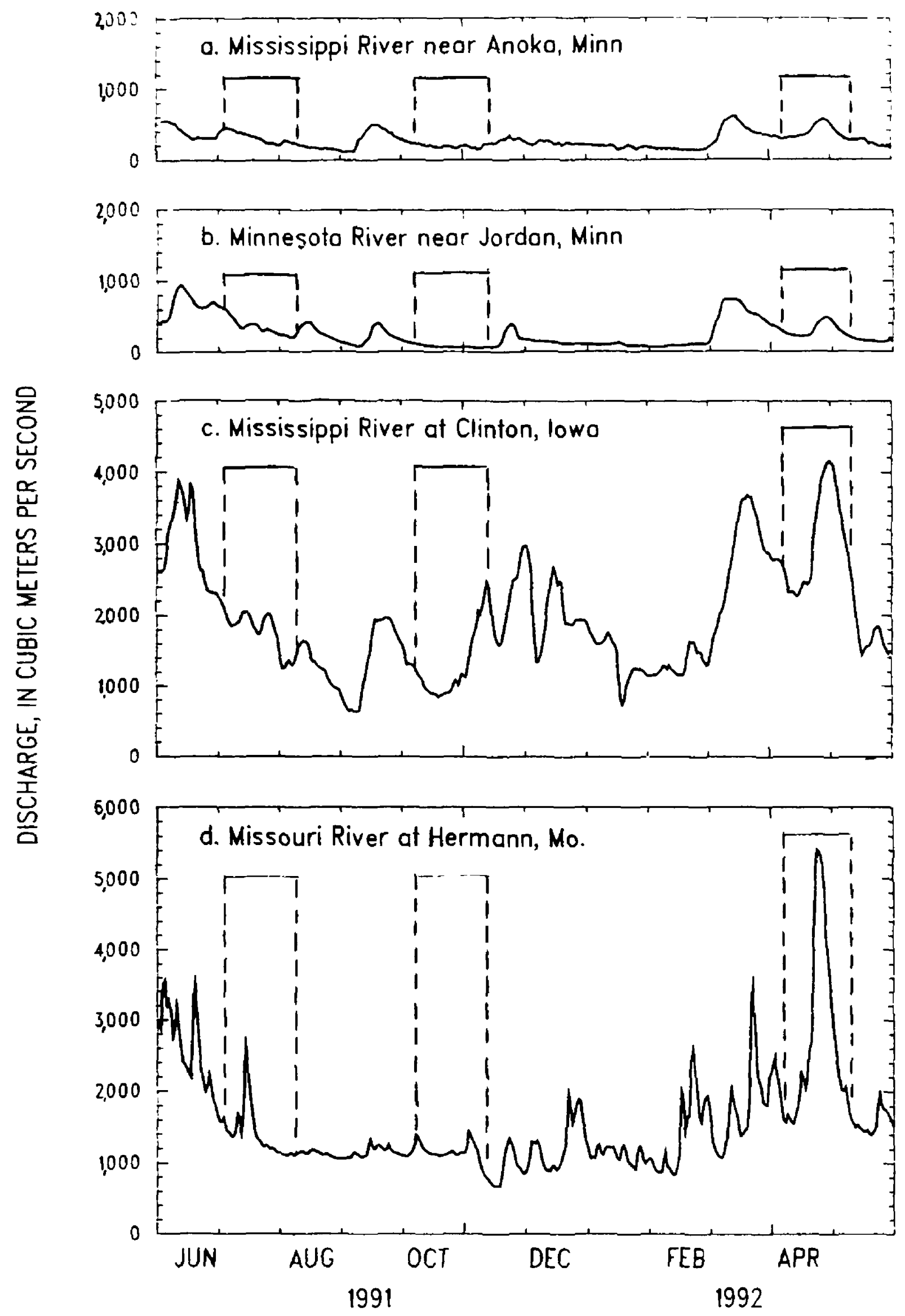

Figure 3.--Water discharge at selected locations upstream from St. Louis, Missouri on the Upper Mississippi River and some of its tributaries from June 1991 through May 1992. The period of time fo- each cruise is shown by two vertical dashed lines. Discharge data are from the U.S. Geological Survey.

(a) The discharge gaging station at Anoka is about 11 kilometers upstream from the samp'ing site above St. Anthony Falls, Minnesota. The mean discharge prior to 1989 is about 230 cubic meters par second (U.S. Geological Survey, 1990d);

(b) The discharge gaging station near Jordan is about 63 kilometers upstream from the Mis The mean discharge prior to 1989 is about 110 cubic meters per second (U.S. Geological Survey, 1990d); (c) The mean discharge at Clinton prior to 1990 is about 1,400 cubic meters per second (U.S. Geological Survey, 1989); and

(d) The discharge gaging station at Hermann is about 158 kilometers upstream from the Mississippi River. The mean discharge prior to 1990 is about 2,300 cubic meters per second (U.S. Geological Survey, 1990c). 

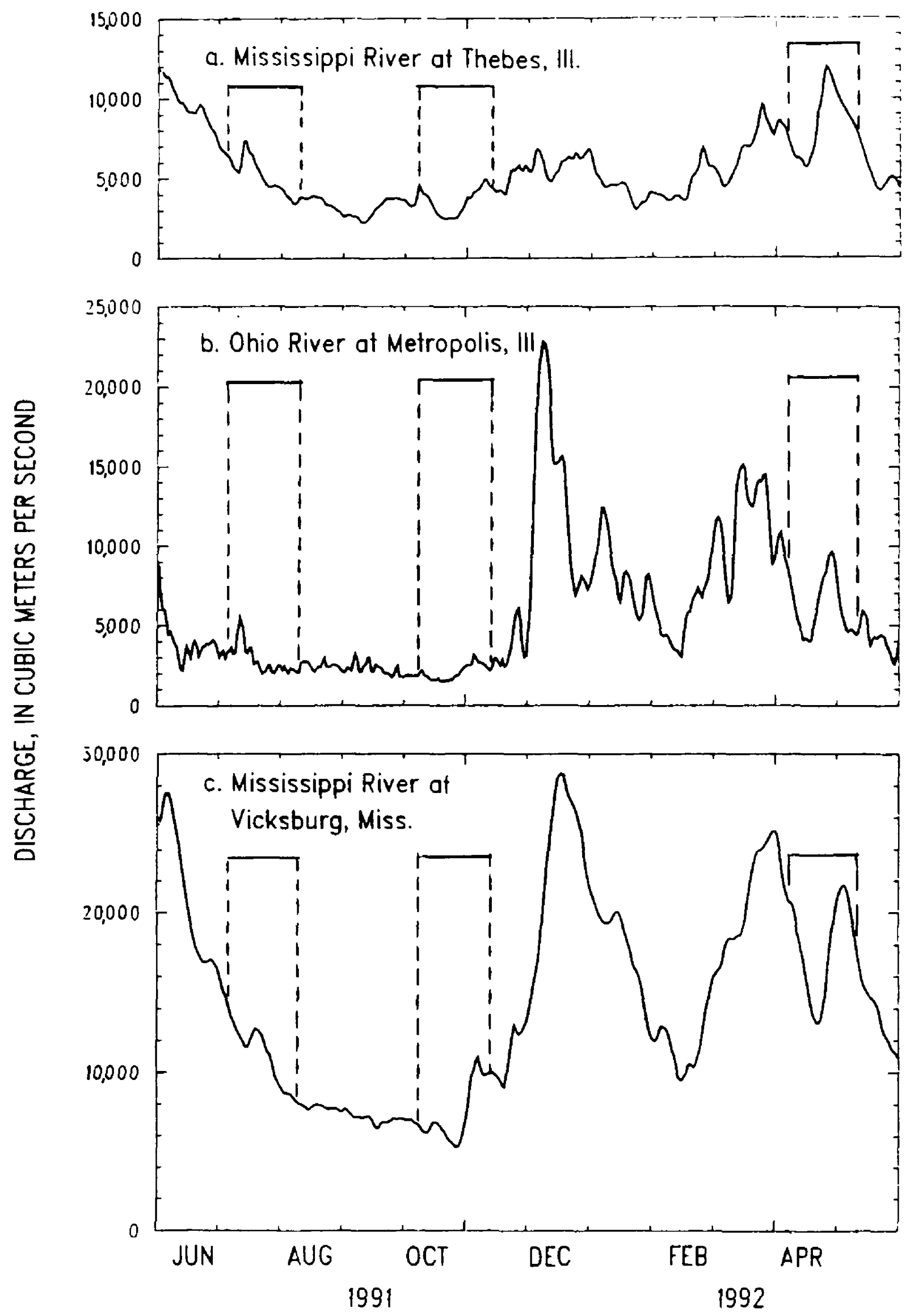

Figure 4.--Water discharge at selected locations downstream from St. Louis, Missouri, on the Upper Mississippi River and some of its tributaries from

June 1991 through May 1992. The period of time for each cruise is shown by two vertical dashed lines. Discharge data are from the U.S. Geological Survey, except for the Mississippi River at Vicksburg, Mississippi (Henry Noble,

U.S. Army Corps of Engineers, Vicksburg District, written commun., 1992).

(a) The mean discharge at Thebes prior to 1990 is about 5,600 cubic meters per second (U.S. Geological Survey, 1990c);

(b) The discharge gaging station at Metropolis is about 29 kilometers upstream from the sampling site at Olmsted,

Illinois. The mean discharge prior to 1990 is about 7,700 cubic meters per second

(U.S. Geological Survey, 1990b); and

(c) The mean discharge at Vicksburg prior to 1990 is about 17,400 cubic meters per second. 


\section{Acknowledoments}

The U.S. Army Corps of Engineers representatives were very accommodating in providing logistic support, overnight docking facilities, and hydrological data. Paul Kornberger, Bill Gretten, and Dennis Erickson ensured that the research vessel was given priority lockage through the 29 locks on the Upper Mississippi River, which allowed the research work to stay on schedule. We wish to especially thank Joe Dvorak, Lockmaster at Lock and Dam No. 1 in Minneapolis, Minnesota, for his help in facilitating crew changes, news conferences, and loading scientific equipment. We also thank James Morgan and the crew at Lock and Dam 15 in Rock Island, Illinois, and Harvey Vance and the crew at Lock and Dam 16 in Muscatine, Iowa, where crew changes, resupply, and repairs were made. Ray Kopsky, St. Louis District; Whit Barton, Memphis District; Henry Noble, Vicksburg District; and Don Flowers with Army Corps Lower Mississippi River Commission, all answered many requests for hydrologic data on short notice.

The U.S. Coast Guard personnel at Keokuk, Iowa, and Hickman, Kentucky, permitt?d the research vessel to dock for the night and provided supplies.

Dan Helwig and Harold Wiegner, Minnesota Pollution Control Agency, provided assistance in planning the initial cruise, and John Sullivan, Wisconsin Department of Natural Resources also provided assistance in planning the research as well as logistical support in LaCrosse, Wisconsin. Rick Bensinger, Waterways Journal, helped in finding fuel for the vessel.

The crew of the research vessel ACADIANA-Wayne Simoneaux, Craig LeBoeuf, Bob Cutting, Cheryl Blanchard, George Collier, Wilton Delaune, Mike Detraz, Derral Dupre, Chuck Guidry, and Jonathan Landry--were especially accommodating and showed an unusuaI interest and concern in maintaining proper scientific operations.

Measuring river discharge or collecting suspended-sediment samples from large rivers is not a twoman operation, and the following people's collaboration was necessary to get the job done: Ron Antweiler, Larry Barber, LaDonna Bishop, Terry Brinton, Greg Brown, Wes Campbel", Gail Chmura, Lesly Conaway, Dolly Dieter, Geoff Ellis, Kathy Fitzgerald, John Garbarino, Don Goolsb.r. Heidi Hayes, Bob Hirsch, Jim Krest, Jerry Leenheer, Gail Mallard, Dick Martin, Stephanie Monsterleet, Ted Noyes, Dale Peart, Ron Rathbun, Dave Roth, Colleen Rostad, Alan Shiller, Mike Simpson, Charles Tabor, Howard Taylor, Lisa Torick, Woodrow Wang, Wayne Webb, and Jeff Writer. 


\section{SAMPLING PROCEDURES AND RESULTS}

The onsite sampling procedures were carried out from the 17-m research vessel ACADIANA, owned and operated by the Louisiana Universities Marine Consortium. This vessel was used because it has a shallow draft (about $1.2 \mathrm{~m}$ ) which permitted samples to be collected close to the river banks. The samples were collected in downstream order from Minneapolis, Minnesota, to New Orleans, Lou'siana, during three cruises, but they probably were not collected as close to a Lagrangian sequence as were the samples from the previous seven cruises (Moody and Meade, 1992, 1993; Moody 1993). The procedures consisted of collecting representative samples of bed sediment, water and suspended sediment, and of measuring physical and basic chemical properties of the water. Bed sediments were sampled at three locations across the navigation channel to provide information for velocity, suspended-sedimert, and bed-load calculations. Bed sediments also were collected from 15-21 locations in approximately the lower one-third of each navigation pool off the main navigation channel. The sampling strategy, particle-size analysis, and chemical analyses of these bed samples are all reported in a sevarate U.S. Geological Survey Open-File Report entitled "Hydrologic, sedimentologic, and chemical data describing water and bed sediments in the navigation pools of the Upper Mississippi River, July 1991April 1992." Suspended sediments were collected at a number of locations or verticals spaced across the river channel by two different methods: (1) discharge-weighted pumping from fixed depths, and (2) depth integration when the water velocity was large enough to flow isokinetically into the sample bottle. For each of the two suspended-sediment sampling methods, the individual samples from each vertical across the river were combined to form two representative samples, which were processed differently-a pumped composite sample and a depth-integrated composite sample. While the depthintegrated sample was being collected, the physical (water depth, depth-integrated or depth-averaged velocity, and surface temperature) and simple chemical (surface $\mathrm{pH}$ and surface specific conductance) properties of the water were measured at each location across the river.

This report is basically organized in the order in which the data are needed to investiga te the movement, mixing, and storage processes of sediment-associated and dissolved pollutants. The detailed bed-sediment procedures and results are presented first because bed sediments affect relocities, suspended-sediment, and bed-load transport. The water-discharge procedures and results are presented next because this information is needed to compute the flux of suspended sediment and dissolved constituents. Suspended-sediment collecting methods, processing procedures, and results follow the "Water-Discharge" section. Also briefly described are the procedures used for concentrating the suspended sediment finer than $63 \mu \mathrm{m}$. These procedures were carried out aboard the ACAD`ANA to reduce the sample volumes to a manageable size that could be shipped to the laboratory for chemical analyses. This report does not include a description of how each of the numerous chemical subsamples from the pumped and depth-integrated composites were prepared, treated, and processed in the laboratory (see Pereira and others, 1995). The last section describes the procedures for determining the suspended-sediment concentration and particle sizes of both the suspended sand and the suspend $\mathrm{d}$ silt and clay fractions. 


\section{Bed Sediments}

A BM-54 sampler (Guy and Norman, 1970, p. 15) was used to collect bed-sediment samples for particle-size analyses. At the beginning of the measurements at each sampling site, while equipment was being set up for the water-discharge measurement and suspended-sediment sampling, the ship drifted downstream and, as it crossed the line of section, a bed-sediment sample was collected. A fathometer (Lowrance, Model X16) was used to obtain a continuous record of depth; at the time each bed sample was taken, a mark was made on this continuous trace of the river bottom. Samples generally were collected at $0.2,0.5$, and 0.8 of the distance between the left and right banks. All particle-size analyses were done at the U.S. Geological Survey sediment laboratory in Iowa, City Iowa. The percent finer than one-half phi sizes (expressed in millimeters) are listed in tables 3, 4, and 5 along with the median diameter determined by straight-line interpolation between appropriate particls sizes listed in tables 3,4 , and 5 . 


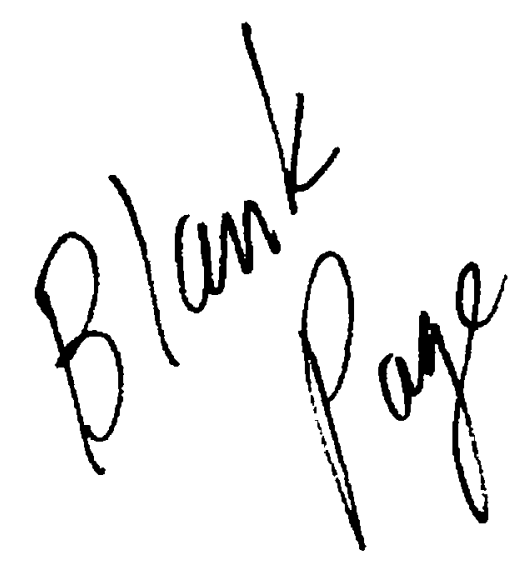

13 


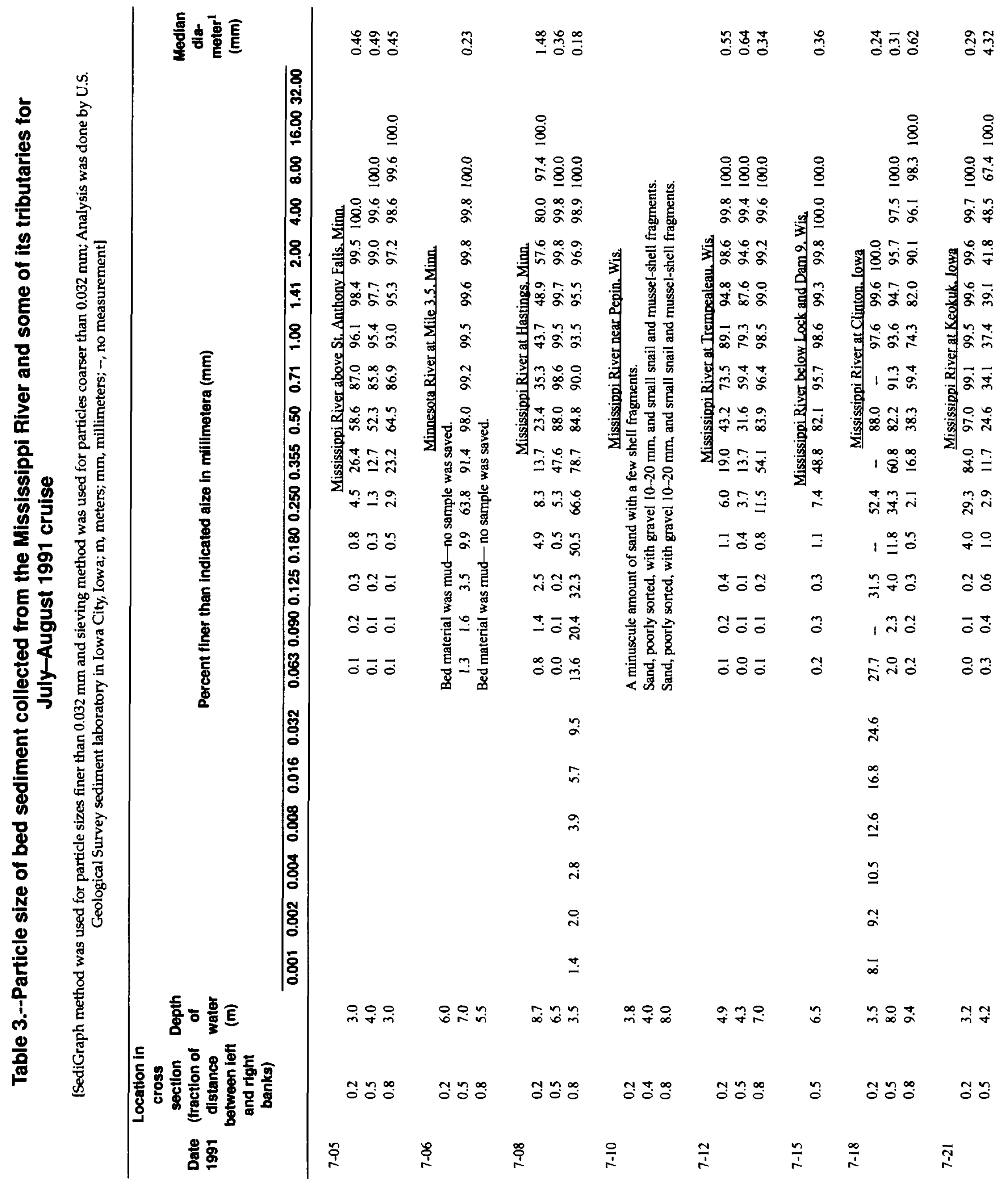






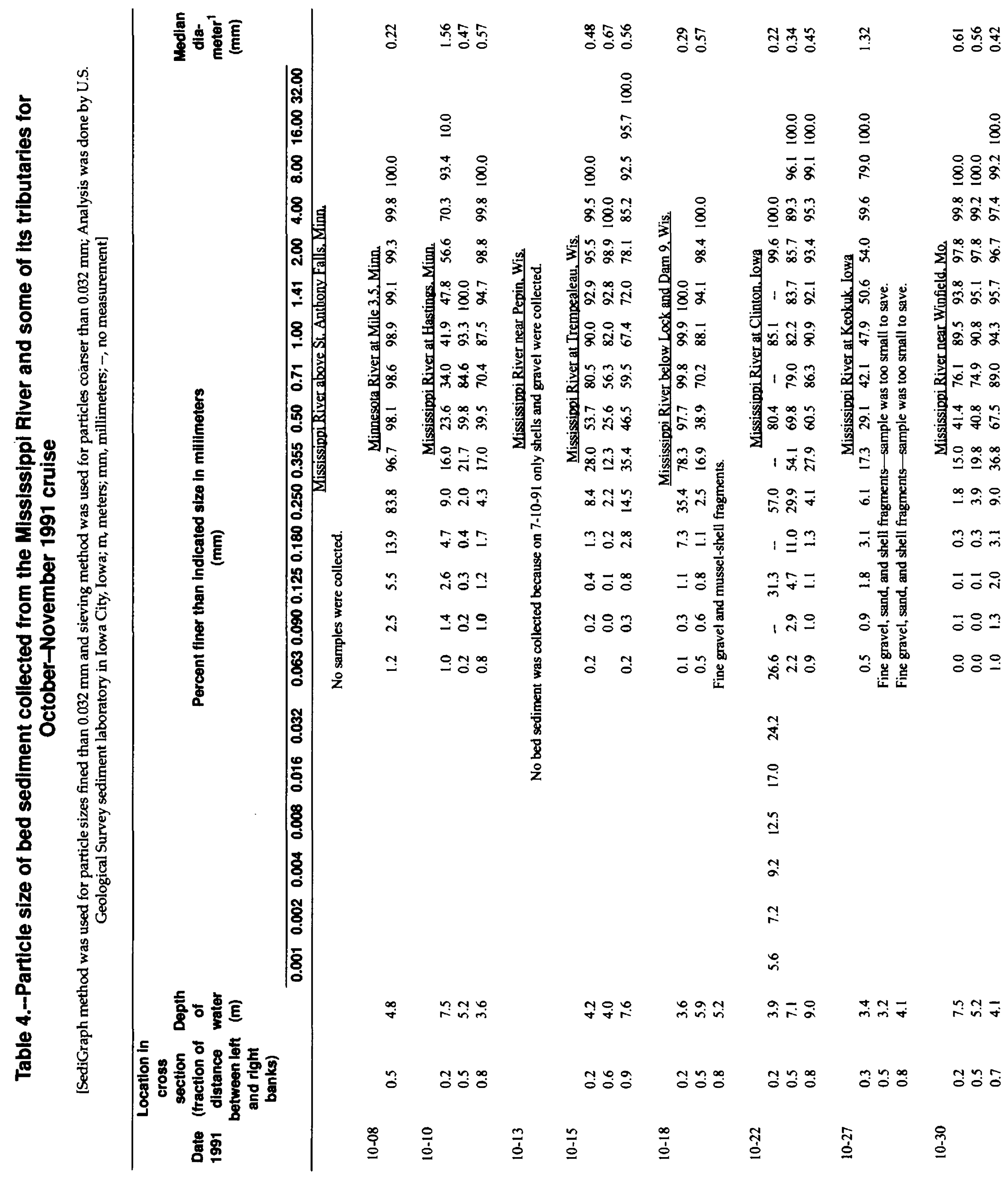




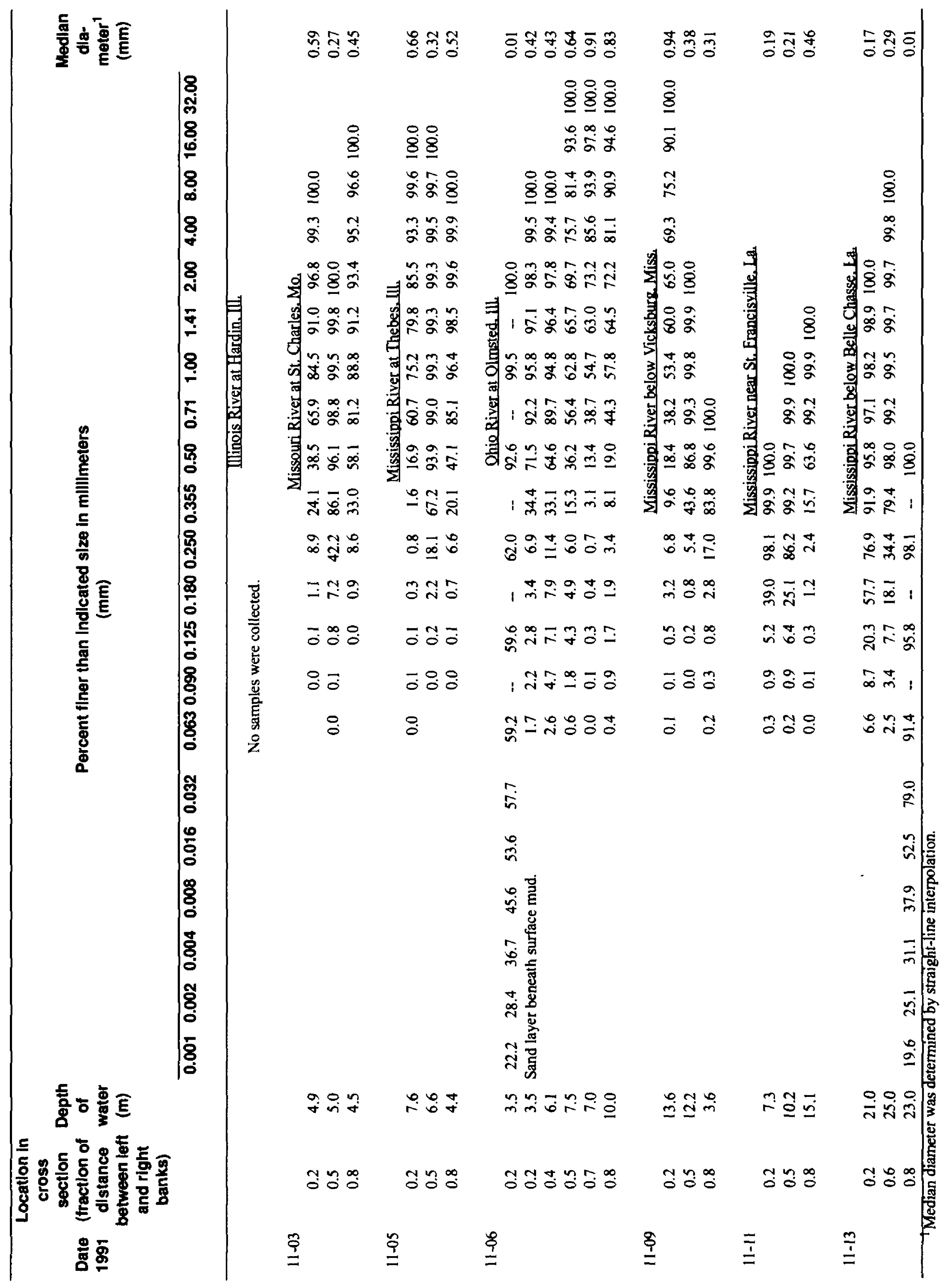




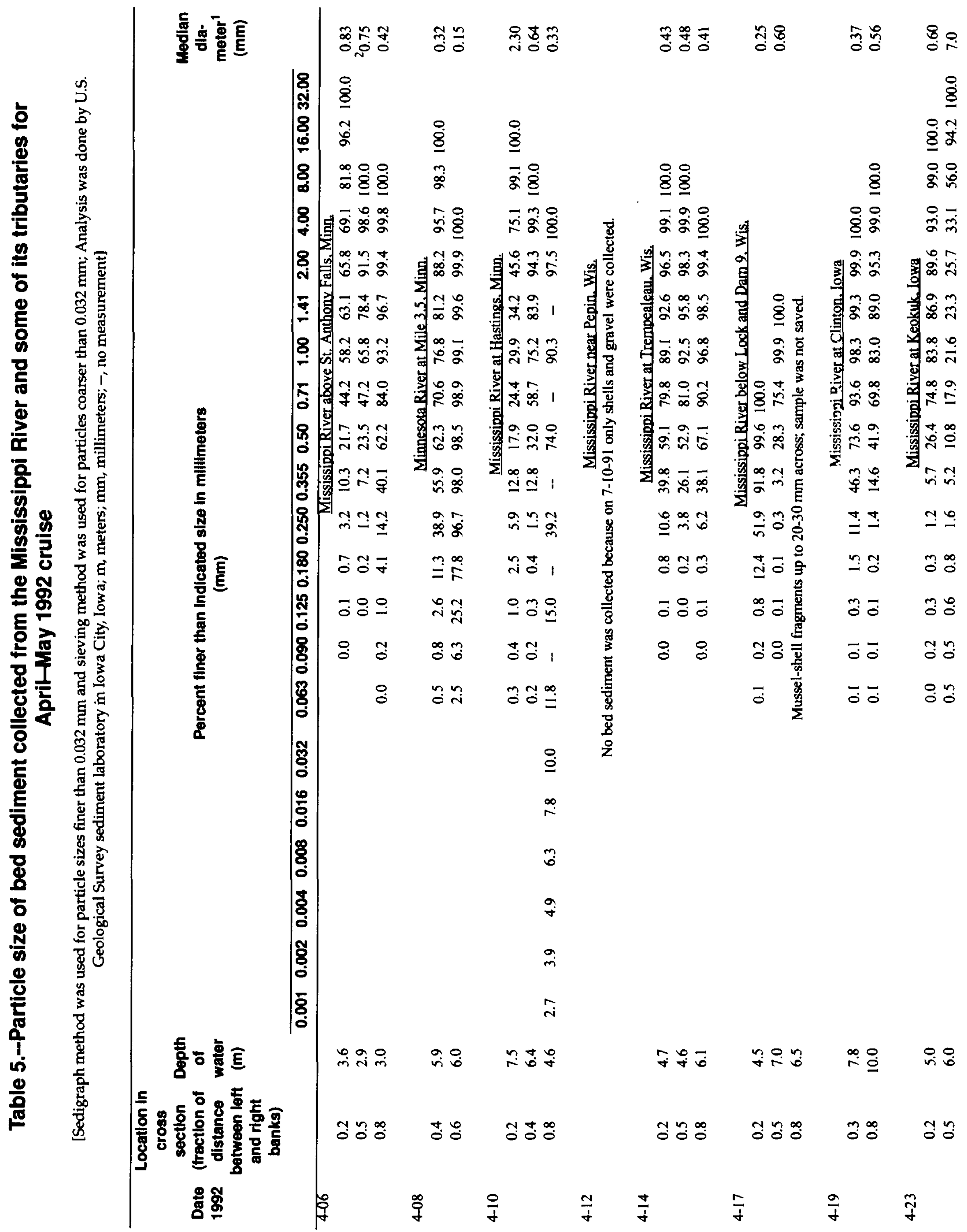



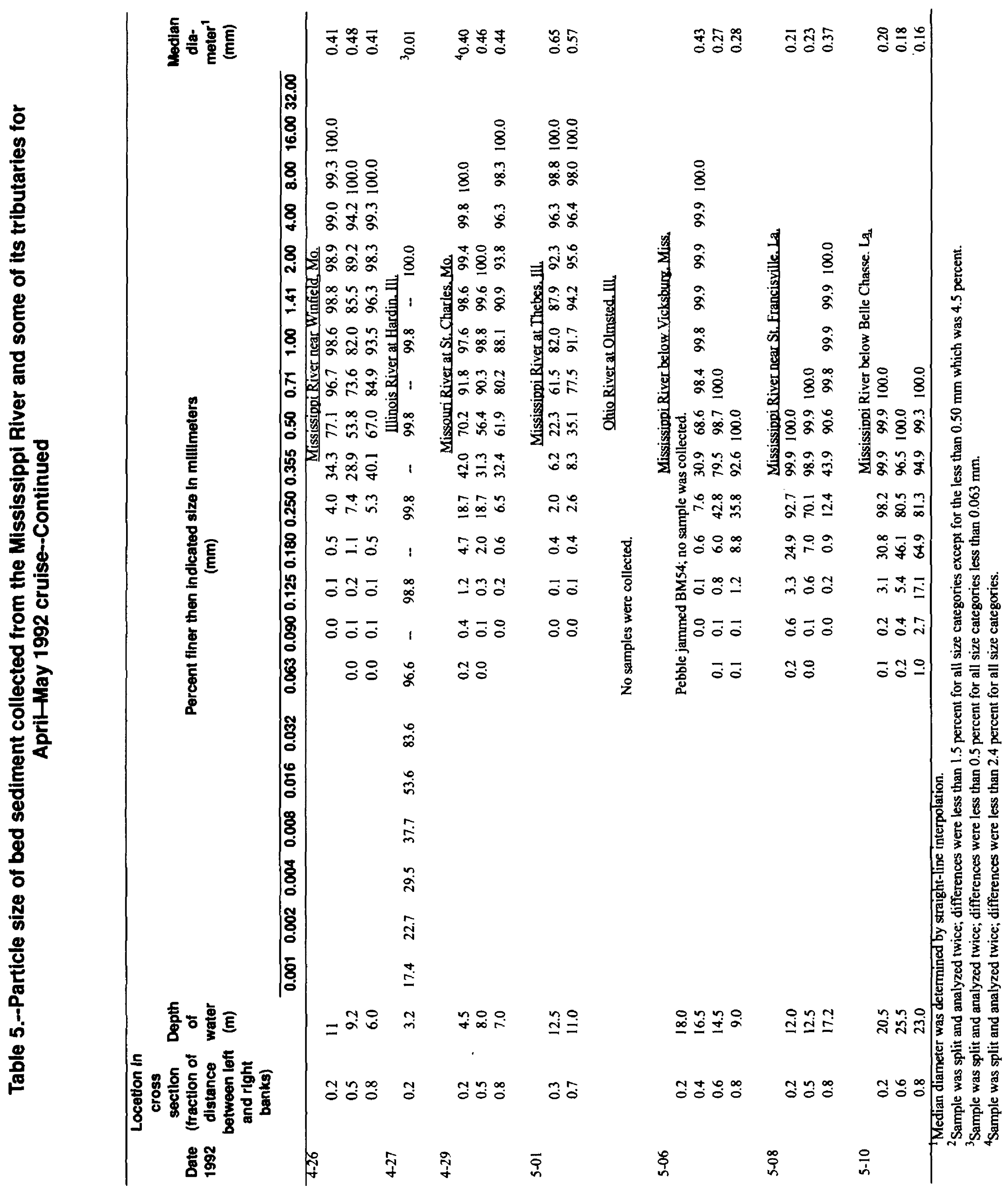


\section{Water Discharge}

The depth-averaged velocity was measured by depth integration from the river bottom to the river surface at several locations (referred to as verticals) across the river. The current meter (Price AA; fig. 5) and suspended-sediment sampler were first lowered to the bottom at a constant transit rate. The depth-averaged velocity was determined only during the upcast when the current meter was not in the turbulent wake of the suspended-sediment sampler bottle. Ideally, for discharge measurements, the transit rate should be as slow as possible and the number of verticals as large as possible so that the integration time is as long as possible. However. at very slow transit rates, the suspended-sediment sampler would overfill. Therefore, the transit rate and number of verticals was determined by the total volume of the composite sample of water and suspended sediment desired (see Nordin and others, 1983), and the transit rate was keft at less than 20 percent of the mean velocity (based on unpublished data from the Amazon Kiver) to obtain a reliable measurement of velocity and, hence, discharge.

Because the ship usually was not anchored during the vertical sampling procedure, the measured velocities were corrected for ship drift according to procedures described by Moody and Troutman (1992). Two microwave transmitter/receiver stations on shore and a maste- station (Del Norte Technology Trisponder system) aboard the ship were used to measure the change in upriver-downriver and cross-river position. The mean depth was obtained from the fatrometer, which produced a continuous strip-chart record of depth during the upcast. The depth-averaged velocity was measured at 4 to 18 equally spaced vertical locations across the river depending upon the width of the river in addition to about 5 measurements made to initially estinate the water discharge (see section titled "Pumped Composite Sample"). These measurements took about 4 hours to complete because extra time was required to repeat some of the depth-intograted water samples and to finish pumping the large water volumes at each vertical.

This method of measuring discharge has associated bias and standard errors, as discussed by Moody and Troutman (1992). Most of the error in the discharge measurement results from errors in measuring the depth-averaged velocity. The major bias is the incomplete depth-interration error (2-5 percent) incurred because the measurement of velocity is started slightly ab vve the bottom (see $h$ in fig. 5). This error can be estimated by assuming a theoretical velocity profile dependent upon the roughness length scale, the water depth, and the height, $h$, of the unmeasured zone. The major source of standard error comes from the natural variability (which is assumed to be random) of river velocities. The standard error of the natural variability is about 5-12 percent of the mean velocity. Typical standard errors in discharge are between 1 and 2 percent. Because the total discharge is essentially a statistically weighted mean of the random velocities, the total discharge error is reduced by the averaging process. The bias and standard errors have been estimated for each discharge measurement according to the methods discussed by Moody and Troutman (1992) (see tables 6-8). The estimated bias error generally is positive (overestimated) because the velocities in the unmeasured zone near the bottom (where velocities are lowest) have not been included in determining the mean velocity. However, at some sections where only a few verticals can be measured, this positive bias error may be smaller than tre negative bias error that results from neglecting the flow between the banks and halfway out to the first or last vertical; thus, the total bias error is negative (for example, see Minnesota River, tables 7 and 8, and Mississippi River at Hastings, Minnesota, in table 7). The standard errors in dischar se were 6 percent or less and averaged 3 percent.

20 HYDROLOGIC AND SEDIMENTOLOGIC DATA COLLECTED DURING THREE CRUISES ON THE MISSISSIPPI RIVER AND SOME OF ITS TRIBUTARIES FROM MINNEAPOLIS, MINNESOTA, TO NEW ORLEANS, LOUISIANA, JULY 1991-MAY 1992 


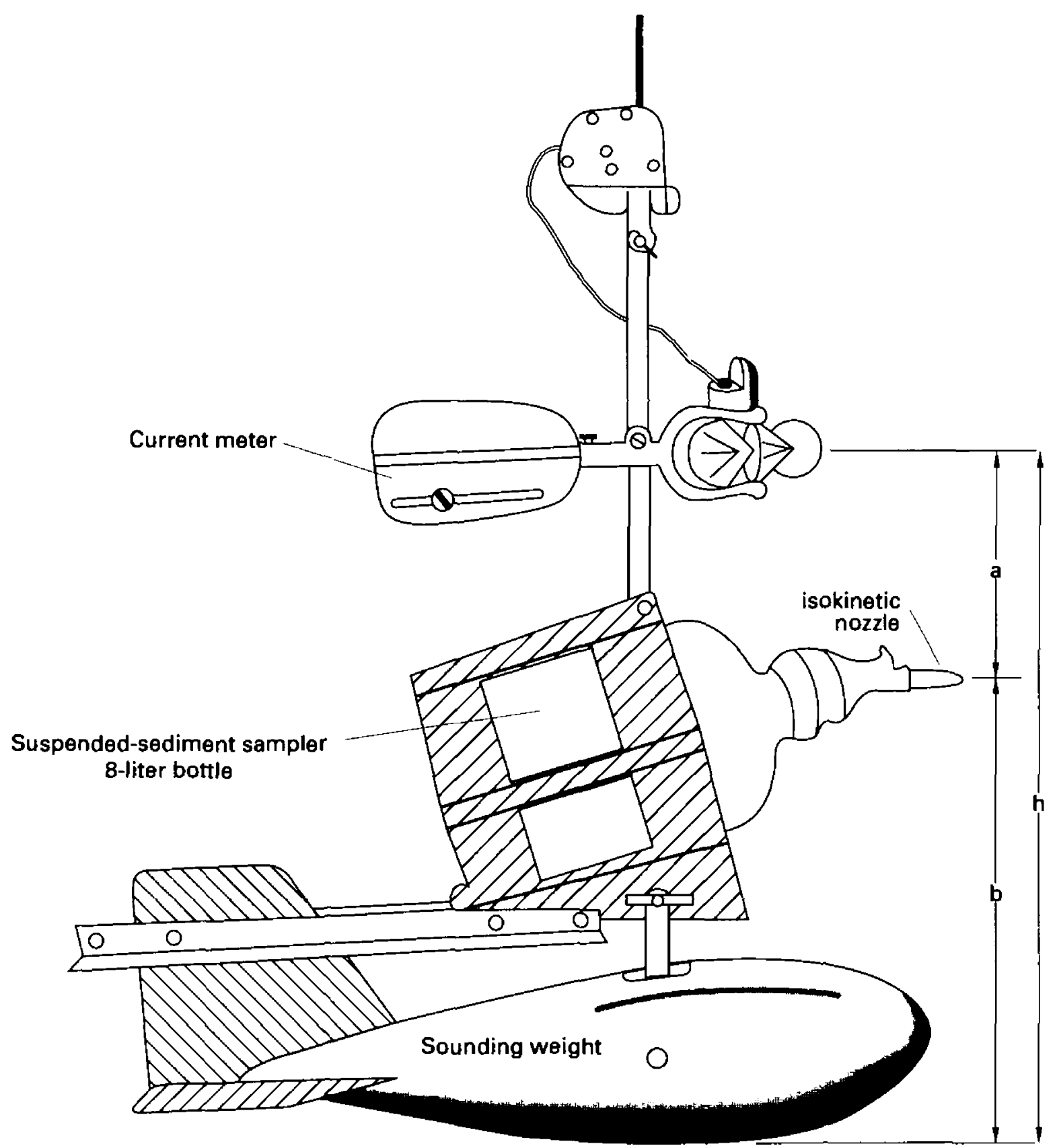

DISTANCE, IN CENTIMETERS

\begin{tabular}{|c|c|c|c|}
\hline WEIGHT, IN POUNDS & $a$ & $b$ & $h$ \\
\hline 150 & 22 & 42 & 64 \\
200 & 22 & 45 & 67 \\
300 & 22 & 48 & 70 \\
\hline
\end{tabular}

Figure 5.--Suspended-sediment sampler with current meter. No water velocity is measured in a zone of height, $h$, above the bottom. 


\section{Tabie 6.-Summary of hydrologlc data for the Mlssissippi River and some of its tr'hutaries for Juiy-August 1991 cruise}

$\left[\mathrm{m}\right.$, meters; $\mathrm{m}^{2}$, square meters; $\mathrm{m} / \mathrm{s}$, meters per second; $\mathrm{m}^{3} / \mathrm{s}$, cubic meters per second; $\%$, percent; NC, not calculated because a different method of measuring discharge was used]

\begin{tabular}{|c|c|c|c|c|c|c|c|c|}
\hline \multirow{4}{*}{$\begin{array}{l}\text { Date } \\
1991\end{array}$} & \multirow{4}{*}{ Site name } & \multirow{4}{*}{$\begin{array}{l}\text { Woth } \\
\text { (m) }\end{array}$} & \multirow{4}{*}{$\begin{array}{c}\text { Cross } \\
\text { section } \\
\text { area } \\
\\
\left(\mathrm{m}^{2}\right)\end{array}$} & \multirow{4}{*}{$\begin{array}{c}\text { Mean } \\
\text { depth } \\
\text { (m) }\end{array}$} & \multirow{4}{*}{$\begin{array}{c}\text { Mean } \\
\text { velocity } \\
(\mathrm{m} / \mathrm{s})\end{array}$} & \multicolumn{3}{|c|}{ Discherge } \\
\hline & & & & & & \multirow{3}{*}{$\begin{array}{l}\text { Magni- } \\
\text { tude } \\
\left(\mathrm{m}^{3} / \mathrm{s}\right)\end{array}$} & \multicolumn{2}{|c|}{ Errors } \\
\hline & & & & & & & Blas 1 & Standard $^{2}$ \\
\hline & & & & & & & $(\%)$ & $(\%)$ \\
\hline 7.05 & $\begin{array}{l}\text { Mississippi R. above } \\
\text { St. Anthony Falls, Minn. }\end{array}$ & 200 & 505 & 2.5 & 0.93 & 470 & 6 & 4 \\
\hline $7-06$ & Minnesota R. at Mile 3.5, Minn. & 109 & 565 & 5.2 & 1.06 & 600 & 4 & 3 \\
\hline $7-08$ & Mississippi R. at Hastings, Minn. & 240 & 1,290 & 5.4 & 0.76 & 980 & 7 & 2 \\
\hline $7-10$ & Mississippi R. near Pepin, Wis. & 466 & 2,140 & 4.6 & 0.63 & 1,350 & 9 & 2 \\
\hline $7-12$ & $\begin{array}{l}\text { Mississippi R. at } \\
\text { Trempealeau, Wis. }\end{array}$ & 435 & 1,950 & 4.5 & 0.74 & 1,440 & 6 & 3 \\
\hline $7-15$ & $\begin{array}{l}\text { Mississippi R. below } \\
\text { Lock and Dam 9, Wis. }\end{array}$ & 450 & 2,150 & 4.8 & 0.74 & 1,590 & 5 & 2 \\
\hline $7-18$ & Mississippi R. at Clinton, lowa & 480 & 2,930 & 6.1 & 0.63 & 1,850 & 5 & 2 \\
\hline $7-21$ & Mississippi R. at Keokuk, lowa & 773 & 2,890 & 3.7 & 0.71 & 2,050 & 6 & 2 \\
\hline $7-24$ & Mississippi R. near Winfield, Mo. & 570 & 3,410 & 6.0 & 0.80 & 2,730 & 5 & 2 \\
\hline $7-25$ & Illinois R. at Hardin, Ill. & 286 & 1,080 & 3.8 & 0.24 & 260 & $\mathrm{NC}$ & NC \\
\hline $7-27$ & Missouri R. at St. Charles, Mo. & 279 & 1,040 & 3.7 & 1.06 & 1,100 & 7 & 2 \\
\hline $7-29$ & Mississippi R. at Thebes, 111. & 584 & 3,960 & 6.8 & 1.11 & 4,390 & 5 & 2 \\
\hline $7-30$ & Ohio R. at Olmsted, III. & 780 & 6,400 & 8.2 & 0.38 & 2,410 & 6 & 2 \\
\hline $8-03$ & $\begin{array}{l}\text { Mississippi R. below } \\
\text { Vicksburg, Miss. }\end{array}$ & 1,176 & 9,380 & 8.0 & 0.93 & 8,750 & 5 & 2 \\
\hline $8-05$ & $\begin{array}{l}\text { Mississippi R. near } \\
\text { St. Francisville, La. }\end{array}$ & 960 & 7,800 & 8.1 & 0.79 & 6,190 & 4 & 2 \\
\hline $8-07$ & $\begin{array}{l}\text { Mississippi R. below } \\
\text { Belle Chasse, La. }\end{array}$ & 720 & 14,450 & 20.1 & 0.30 & 4,340 & -2 & 5 \\
\hline
\end{tabular}

${ }^{1}$ The bias error is the sum of errors resulting from not making velocity measurements within a distance above the bottom equal to the distance from the current meter to the bottom of the sounding weight (positive bias) and from neglecting the flow along the bank (negative bias) for a distance out from the bank equal to one-half of the distance between the first or last vertical and the edge of water.

${ }^{2}$ The standard ęrror is primarily the sum of errors resulting from the velocity variability inherent in the fluid flow of all rivers and the error resulting from using a finite number of verticals to approximate a continuous variation in discharge across a river. 
Table 7.--Summary of hydrologic data for the Mlssissippl River and some of its tributarins for October-November 1991 cruise

[m, meters; $\mathrm{m}^{2}$, square meters; $\mathrm{m} / \mathrm{s}$, meters per second; $\mathrm{m}^{3} / \mathrm{s}$, cubic meters per second; $\%$, percent]

\begin{tabular}{|c|c|c|c|c|c|c|c|c|}
\hline \multirow{3}{*}{$\begin{array}{l}\text { Date } \\
1991\end{array}$} & \multirow{3}{*}{ Site name } & \multirow{3}{*}{$\begin{array}{l}\text { Width } \\
\text { (m) }\end{array}$} & \multirow{3}{*}{$\begin{array}{l}\text { Cross } \\
\text { sectlon } \\
\text { area } \\
\left(m^{2}\right)\end{array}$} & \multirow{3}{*}{$\begin{array}{l}\text { Moan } \\
\text { depth } \\
\text { (m) }\end{array}$} & \multirow{3}{*}{$\begin{array}{c}\text { Moan } \\
\text { velocity } \\
(\mathrm{m} / \mathrm{s})\end{array}$} & \multicolumn{3}{|c|}{ Dlscharge } \\
\hline & & & & & & \multirow{2}{*}{$\begin{array}{l}\text { Magni- } \\
\text { tude } \\
\left(\mathrm{m}^{3} / \mathrm{s}\right)\end{array}$} & \multicolumn{2}{|c|}{ Errors } \\
\hline & & & & & & & $\begin{array}{c}\text { Blas' } \\
(\%)\end{array}$ & $\begin{array}{c}\text { Standard } \\
(\%)\end{array}$ \\
\hline $10-07$ & $\begin{array}{l}\text { Mississippi R. above } \\
\text { St. Anthony Falls, Minn. }\end{array}$ & 163 & 423 & 2.6 & 0.52 & 220 & 13 & 5 \\
\hline $10-08$ & Minnesota R. at Mile 3.5, Minn. & 103 & 384 & 3.7 & 0.33 & 130 & -2 & 5 \\
\hline $10-10$ & Mississippi R. at Hastings, Minn. & 215 & 938 & 4.4 & 0.37 & 350 & -4 & 5 \\
\hline $10-13$ & Mississippi R. near Pepin, Wis. & 440 & 1,610 & 3.7 & 0.32 & 510 & 4 & 5 \\
\hline $10-15$ & Mississippi R. at Trempealeau, Wis. & 430 & 1,740 & 4.0 & 0.38 & 660 & 0 & 4 \\
\hline $10-18$ & $\begin{array}{l}\text { Mississippi R. below } \\
\text { Lock and Dam 9. Wis. }\end{array}$ & 444 & 1,720 & 3.9 & 0.40 & 690 & -2 & 5 \\
\hline $10-22$ & Mississippi R. at Clinton, lowa & 450 & 2,540 & 5.6 & 0.37 & 940 & -2 & 4 \\
\hline $10-27$ & Mississippi R. at Keokuk, lowa & 745 & 2,200 & 3.0 & 0.64 & 1,410 & -2 & 4 \\
\hline $10-30$ & Mississippi R. near Winfield, Mo. & 563 & 2,630 & 4.7 & 0.47 & 1,230 & -1 & 4 \\
\hline $10-31$ & 11linois R. at Hardin, 111. & 270 & 1,120 & 4.1 & 0.47 & 520 & 2 & 3 \\
\hline $11-03$ & Missouri R. at St. Charles, Mo. & 280 & 1,170 & 4.2 & 1.15 & 1,350 & 5 & 3 \\
\hline $11-05$ & Mississippi R. at Thebes, Ill. & 590 & 3.360 & 5.7 & 1.15 & 3,870 & 5 & 2 \\
\hline $11-06$ & Ohio R. at Olmsted, 111. & 962 & 5,810 & 6.0 & 0.43 & 2,480 & 1 & 3 \\
\hline $11-09$ & $\begin{array}{l}\text { Misstssippi R. below } \\
\text { Vicksburg, Miss. }\end{array}$ & 1,180 & 10,630 & 9.0 & 1.01 & 10,700 & 5 & 2 \\
\hline $11-11$ & $\begin{array}{l}\text { Mississippi R. near } \\
\text { St. Francisville, La. }\end{array}$ & 974 & 9,170 & 9.4 & 0.98 & 8,950 & 3 & 2 \\
\hline $11-13$ & $\begin{array}{c}\text { Mississippi R. below } \\
\text { Belle Chasse, La. }\end{array}$ & 781 & 15,810 & 20.2 & 0.56 & 8,840 & 0 & 4 \\
\hline
\end{tabular}

${ }^{1}$ The bias error is the sum of errors resulting from not making velocity measurements within a distance above the bottom equal to the distznce from the current meter to the bottom of the sounding weight (positive bias) and from neglecting the flow along the bank (negative bias) for a distance out from the bank equal to one-half of the distance between the first or last vertical and the edge of water.

${ }^{2}$ The standard error is primarily the sum of errors resulting from the velocity variability inherent in the fluid flow of all rivers and the error resulting from using a finite number of verticals to approximate a continuous variation in discharge across a river. 


\section{Table 8.--Summary of hydrologic data for the Mississippi River and some of its tributaries for Apri-May 1992 cruise}

[m, meters; $\mathrm{m}^{2}$, square meters; $\mathrm{m} / \mathrm{s}$, meters per second; $\mathrm{m}^{3} / \mathrm{s}$, cubic meters per second; \%, percent; NC, not calculated because a different method of measurin $\%$ discharge was used]

\begin{tabular}{|c|c|c|c|c|c|c|c|c|}
\hline \multirow{3}{*}{$\begin{array}{l}\text { Date } \\
1992\end{array}$} & \multirow{3}{*}{ Site name } & \multirow{3}{*}{$\begin{array}{l}\text { Width } \\
\text { (m) }\end{array}$} & \multirow{3}{*}{$\begin{array}{c}\text { Cross } \\
\text { section } \\
\text { area } \\
\\
\left(\mathbf{m}^{2}\right)\end{array}$} & \multirow{3}{*}{$\begin{array}{l}\text { Mean } \\
\text { depth } \\
\text { (m) }\end{array}$} & \multirow{3}{*}{$\begin{array}{c}\text { Mean } \\
\text { velocity } \\
(\mathrm{m} / \mathrm{s})\end{array}$} & \multicolumn{3}{|c|}{ Discharge } \\
\hline & & & & & & \multirow{2}{*}{$\begin{array}{l}\text { Magni- } \\
\text { tude }\end{array}$} & \multicolumn{2}{|c|}{ Errors } \\
\hline & & & & & & & $\begin{array}{c}\text { Bias }^{1} \\
(\%)\end{array}$ & $\begin{array}{c}\text { St rndard } \\
\text { (\%) }\end{array}$ \\
\hline $4-06$ & $\begin{array}{l}\text { Mississippi R. above } \\
\text { St. Anthony Falls, Minn. }\end{array}$ & 168 & 515 & 3.1 & 0.60 & 310 & 12 & 3 \\
\hline $4-07$ & $\begin{array}{l}\text { Mississippi R. at } \\
\text { Mile 844.0, Minn. }\end{array}$ & 145 & 706 & ${ }^{3} 4.9$ & 0.51 & 360 & -3 & 5 \\
\hline 4-08 & Minnesota $R$. at Mile 3.5, Minn. & 93 & 419 & 4.5 & 0.62 & 260 & -7 & 6 \\
\hline $4-07$ & Minnesota $R$. at Mile 0.0, Minn. & 141 & 411 & ${ }^{3} 2.9$ & 0.75 & 310 & -10 & 6 \\
\hline $4-10$ & Mississippi R. at Hastings, Minn. & 225 & 1,140 & 5.0 & 0.50 & 570 & 7 & 3 \\
\hline $4-11$ & $\begin{array}{l}\text { St. Croix R. at } \\
\text { Mile 0.0, Minn/Wis. }\end{array}$ & 152 & 994 & 6.5 & 0.32 & 320 & NC & $N^{\prime} \mathrm{C}$ \\
\hline 4- 12 & Mississippi R. near Pepin, Wis. & 460 & $\mathrm{I}, 970$ & 4.3 & 0.48 & 950 & 5 & 4 \\
\hline $4-12$ & Chippewa $R$. at Mile 2.0 , Wis. & 212 & 317 & 1.5 & 0.95 & 300 & $\mathrm{NC}$ & $N^{\prime} \mathrm{C}$ \\
\hline 4-14 & $\begin{array}{l}\text { Mississippi R. at } \\
\text { Trempealeau, Wis. }\end{array}$ & 428 & 2,010 & 4.7 & 0.66 & 1,330 & 6 & 2 \\
\hline 4-17 & $\begin{array}{l}\text { Mississippi R. below } \\
\text { Lock and Dam 9, Wis. }\end{array}$ & 450 & 2,260 & 5.0 & 0.70 & 1,590 & 5 & 2 \\
\hline $4-19$ & Mississippi R. at Clinton, lowa & 475 & 3,250 & 6.8 & 0.71 & 2,320 & 5 & 2 \\
\hline $4-23$ & Mississippi R. at Keokuk, lowa & 790 & 4,030 & 5.1 & 1.05 & 4,220 & 5 & 2 \\
\hline 4-26 & Mississippi R. near Winfield, Mo. & 580 & 4,560 & 7.9 & 1.11 & 5,070 & 5 & 2 \\
\hline $4-27$ & Illinois R.at Hardin, III. & 277 & 1,290 & 4.7 & 0.66 & 860 & 8 & 3 \\
\hline 4-29 & Missouri R. at St. Charles, Mo. & 415 & 2,310 & 5.6 & 1.54 & 3,560 & 5 & 2 \\
\hline $5-01$ & Mississippi R. at Thebes, 111. & 617 & 6,320 & 10.2 & 1.66 & 10,500 & 3 & 2 \\
\hline $5-03$ & Ohio R. at Olmsted, 11 . & 1,012 & 9,980 & 9.9 & 0.62 & 6,150 & 2 & 2 \\
\hline $5-06$ & $\begin{array}{l}\text { Mississippi R. below } \\
\text { Vicksburg, Miss. }\end{array}$ & 1,213 & 15,820 & 13.0 & 1.37 & 21,700 & 3 & 1 \\
\hline $5-08$ & $\begin{array}{l}\text { Mississippi R. near } \\
\text { St. Francisville, La. }\end{array}$ & 1,015 & 12,750 & 12.6 & 1.18 & 15,100 & 3 & 2 \\
\hline $5-10$ & $\begin{array}{c}\text { Mississippi R, below } \\
\text { Belle Chasse, La. }\end{array}$ & 795 & 16,350 & 20.6 & 0.89 & 14,500 & 3 & 2 \\
\hline
\end{tabular}

\footnotetext{
The bias error is the sum of errors resulting from not making velocity measurements within a distance above the bottom equal to the distance from the current meter to the bottom of the sounding weight (positive bias) and from neglecting the flow along the bank (negative bias) for a distance out from the bank equal to one-half of the distance between the first or last vertical and the edge of water.

2 The standard error is primarily the sum of errors resulting from the velocity variability inherent in the fluid flow of all rivers and the error resulting from using a finite number of verticals to approximate a continuous variation in discharge across a river.

${ }^{3}$ Mean depth is biased toward shallow depth because only 4.5 equally spaced verticals were used to measure the discharge.
} 
The nearly instantaneous (about 4 hours measurement time) discharge values measured by depth integration on each cruise were compared with the published daily mean discharge values for gaging stations near or at five of the sampling sites (Mississippi River above St. Anthony Falls, Minnesota; at Clinton, Iowa; at Thebes, Illinois; below Vicksburg, Mississippi; and Ohio River at Olmsted, Illinois). The discharges ranged from 220 to $21,700 \mathrm{~m}^{3} / \mathrm{s}$, the 15 differences between the published discharge values and those we measured ranged from -10 to 9 percent. The average depth-integrated dis - harge was 0.3 percent greater than the published daily mean discharge.

The water discharge of the tributaries (where only surface samples were collected within 2-3 km of the mouth of the St. Croix, Chippewa, Wisconsin, Rock, lowa, Des Moines, Kaskaskia, White, Arlansas, and Yazoo Rivers) was assumed to be equal to the daily mean discharge for the gaging station clcsest to the mouth of the tributary for the day the tributary was sampled. The discharges for these tributary sites and the discharges measured by the depth-integration method for the other sampling sites have been summarized for all three cruises in table 9. 


\section{Table 9.-Summary of water discharges for the Mississippi River and some of its tibutaries for three cruises}

[Discharges are for the day the site was sampled which is listed in tables 6-8; River miles are given for the mouth of the tributary; $\mathrm{m}^{3} / \mathrm{s}$, cubic meters per serond; -- , does not apply]

\begin{tabular}{|c|c|c|c|c|c|}
\hline \multirow[b]{2}{*}{ Mississippl and tributary sites } & \multicolumn{2}{|c|}{ Rlver miles } & \multicolumn{3}{|c|}{$\begin{array}{l}\text { Water disch } \times r g e \\
\left(\mathrm{~m}^{3} / \mathrm{s}\right)\end{array}$} \\
\hline & $\begin{array}{l}\text { Upriver } \\
\text { from } \\
\text { mouth of } \\
\text { Ohlo R. }\end{array}$ & $\begin{array}{l}\text { Upriver } \\
\text { from } \\
\text { Head of } \\
\text { Passes }\end{array}$ & $\begin{array}{c}\text { July- } \\
\text { August } \\
1991 \\
\text { crulse }\end{array}$ & $\begin{array}{c}\text { October- } \\
\text { Novembrer } \\
1991 \\
\text { crulse }\end{array}$ & $\begin{array}{c}\text { Aprit- } \\
\text { May } \\
1992 \\
\text { cruise }\end{array}$ \\
\hline Mississippi R. above St. Anthony Falls, Minn. & 857.7 & $1,811.5$ & 470 & 220 & 310 \\
\hline Minnesota R. at Mile 3.5, Minn. & 844.0 & $1,797.8$ & 600 & 130 & $5_{260}$ \\
\hline Mississippi R. at Hastings, Minn. & 812.2 & $1,766.0$ & 980 & 350 & 570 \\
\hline St. Croix R. at St. Croix Falls, Wis. ${ }^{\prime}$ & 811.3 & $1,765.1$ & 260 & 95 & ${ }^{5} 320$ \\
\hline Mississippi R. near Pepin, Wis. & 764.5 & $1,718.3$ & 1,350 & 510 & 950 \\
\hline Chippewa R. at Durand, Wis.' & 763.5 & $1,717.3$ & 160 & 161 & $5_{300}$ \\
\hline Mississippi R. at Trempealeau, Wis. & 713.8 & $1,667.6$ & 1,440 & 660 & 1,330 \\
\hline Mississippi R. below Lock and Dam 9, Wis. & 639.7 & $1,593.5$ & 1,590 & 690 & 1,590 \\
\hline Wisconsin R. at Muscoda, Wis. ${ }^{1}$ & 630.6 & $1,584.4$ & 145 & 161 & 368 \\
\hline Mississippi R. at Clinton, lowa & 520.3 & $1,474.1$ & 1,850 & 940 & 2,320 \\
\hline Rock R. near Joslin, III.' & 479.0 & $1,432.8$ & 68 & 82 & 337 \\
\hline lowa R. at Wapello, lowa' & 434.0 & $1,387.8$ & 200 & 74 & 685 \\
\hline Mississippi R. at Keokuk, Iowa & 363.1 & $1,316.9$ & 2,050 & 1,410 & 4,220 \\
\hline Des Moines R. at Keosauqua, Iowa' & 361.5 & $1,315.3$ & 623 & 39 & 719 \\
\hline Mississippi R. near Winfield, Mo. & 239.2 & $1,193.0$ & 2,730 & 1,230 & 5,070 \\
\hline Illinois R. at Hardin, Ill. & 218.0 & $1,171.8$ & 260 & 520 & 860 \\
\hline Missouri R. at St. Charles, Mo. & 195.3 & $1,149.1$ & 1,100 & 1,350 & 3,560 \\
\hline Kaskaskia R. near Venedy Station, III. I & 117.3 & $1,071.1$ & 7 & 10 & 31 \\
\hline Mississippi R. at Thebes, Ill. & 44.0 & 997.8 & 4,390 & 3,870 & 10,500 \\
\hline Ohio R. at Olmsted, III. & 0 & 953.8 & 2,410 & 2,480 & 6,150 \\
\hline White R. at Clarendon, Ark. ${ }^{2}$ & -- & 598.8 & 374 & 1,220 & 920 \\
\hline Arkansas R. at Dam 2. Ark. ${ }^{3}$ & $\cdots$ & 581.5 & 480 & 1,620 & 710 \\
\hline Yazoo R. below Steele Bayou, Miss. ${ }^{4}$ & -- & 437.2 & 640 & 540 & $\sim 70$ \\
\hline Mississippi R. below Vicksburg, Miss. & -- & 433.4 & 8,750 & 10,700 & 21,700 \\
\hline Mississippi R. near St. Francisville, La. & $\cdots$ & 266.4 & 6,190 & 8,950 & 15,100 \\
\hline Mississippi R. below Belle Chasse, La. & -- & 73.1 & 4,340 & 8,840 & 14,500 \\
\hline
\end{tabular}

\footnotetext{
'Daily mean discharge based on U.S. Geological Survey discharge for the gaging station closest to the mouth of the tributary.

${ }^{2}$ Discharge provided by U.S. Army Corps of Engineers, Memphis District for White River at Clarendon, Arkansas-about 142 kilometers upstream from the mouth.

${ }^{3}$ Discharge at 0800 hours over Dam 2 on the Arkansas River-approximately 56 kilometers upriver from the mouth.

${ }^{4}$ Discharge is only an estimate based on the measurement of discharge at Redwood, Mississippi, that is closest in time to the sampling date plus discharge at Steele Bayou.

${ }^{5}$ Discharge was measured at the mouth.
} 


\section{Water and Suspended Sediment}

Two methods were used for collecting composite water and suspended-sediment samples. One method was the pumping method from fixed depths, which yielded large volumes (about 500-1.000L) of water and a representative sample of particles finer than $63 \mu \mathrm{m}$ (Moody and Meade, 1994). The pumping method also could be used to collect smaller volumes when the water velocity was ton slow for using the depth-integration method. The depth-integration method yielded relatively smaller volumes of water but representative samples of both the sand fraction (greater than $63 \mu \mathrm{m}$ ) and the silt and clay fraction (finer than $63 \mu \mathrm{m}$ ) of suspended sediment. This method could not be used to collect a representative sample when the water velocity was less than about $50 \mathrm{~cm} / \mathrm{s}$, because the sampling efficiency became unpredictable. Different procedures were used to process the pumped and deptr integrated composite samples after collection.

\section{Pumped Composile Sample}

The pumped composite sample was collected by using a 12-mm-diameter, perflourinated-alkoxy (PFA) Teflon tube inside a $12.7-\mathrm{mm}$-diameter, double-braided stainless-steel hose, which was att*ched to a 300-pound sounding weight to keep the nozzle directed into the current. A hydraulic winch was used to lower the nozzle and sounding weight at each vertical to a depth of $5 \mathrm{~m}$ below the surface or one-half the water depth (whichever was less). Water was pumped from the fixed depth by a doublediaphragm pump (Wilden MI/UP/TF/TF/TF), driven by compressed air and constructed entirely of PFA Teflon, through a $63-\mu \mathrm{m}$ sieve and into a calibrated funnel (45-L upside-down carboy w:th no bottom).

The sand that collected in the 63- $\mu \mathrm{m}$ sieve was called the pumped, composite sand sample, and the water and remaining suspended sediment was called the pumped, composite silt, clay, and colloid sample. The volume of river water that was pumped at each vertical was proportional to the estimated fractional water discharge at each vertical. The following steps (described in greater detail by Moody and Meade, 1994) were used to estimate the volume of water to be pumped at each vertical:

1. Determine the depth of each vertical (where water will be pumped) from the continuous stripchart record of the bottom provided by the fathometer;

2. Measure a few depth-averaged velocities at three to five locations with different depths;

3. Calculate the proportionality constant relating depth-averaged velocity to the depth to the 2/3 power (Manning's formula, Chow, 1959; Sium, 1975; and Sayre and Caro-Cordero, 1977);

4. Interpolate the value of the proportionality constant at verticals (where water will be purnped) between the three to five locations where depth-averaged velocities were measured;

5. Compute the discharge, centered about each vertical, and its fraction of the total discharge: and

6. Calculate the volume to be pumped at each vertical as the fraction of the total discharge at each vertical times the total volume to be pumped.

The total volume of river water collected by the pumping method is listed in table 10 for the JulyAugust 1991 cruise, in table 11 for the October-November 1991 cruise, and in table 12 for the April-May 1992 cruise. 
Table 10.--Volumes of river water collected and processed, assoclated samplinc parameters, and percent recovery of suspended sediment for the July-August 1991 cruise

$\left[\mathrm{V}_{\mathrm{c}}\right.$, total volume collected; $\mathrm{V}_{\mathrm{d}}$, volume centrifuged; $\mathrm{V}_{\mathrm{u}}$, volume ultrafiltered; $\mathrm{L}$, liters; $\mathrm{cm}$ / $s$, centimeters per second; $\mu \mathrm{m}$, micrometer; $\%$, percent; Integration, depth-integration; Pumped, discharge-weighted pumping from fixed depths; Dipped, surface dip sample at one location; --, dashes mean does not apply]

\begin{tabular}{|c|c|c|c|c|c|c|c|c|c|}
\hline \multirow[b]{2}{*}{$\begin{array}{l}\text { Date } \\
1991\end{array}$} & \multirow[b]{2}{*}{ Slte name } & \multirow[b]{2}{*}{$\begin{array}{l}\text { Collecting } \\
\text { method }\end{array}$} & \multicolumn{3}{|c|}{ Sample volumes } & \multicolumn{3}{|c|}{ Sampling parameters } & \multirow{2}{*}{$\begin{array}{l}\text { Recovery } \\
\text { of } \\
\text { suspended } \\
\text { sediment } \\
\text { less than } \\
63 \mu \mathrm{m}^{1} \\
(\%)\end{array}$} \\
\hline & & & $\begin{array}{l}\mathbf{v}_{c} \\
(L)\end{array}$ & $\begin{array}{l}V_{d} \\
(L)\end{array}$ & $\begin{array}{l}\mathbf{v}_{\mathbf{u}} \\
(\mathrm{L})\end{array}$ & $\begin{array}{l}\text { Num- } \\
\text { ber } \\
\text { of } \\
\text { verti- } \\
\text { cals }\end{array}$ & $\begin{array}{c}\text { Nozzle } \\
\text { size } \\
\text { (Inches) }\end{array}$ & $\begin{array}{c}\text { Tran rit } \\
\text { rat: } \\
(\mathrm{cm} / \mathrm{s})\end{array}$ & \\
\hline $7-05$ & $\begin{array}{l}\text { Mississippi R. above } \\
\text { St. Anthony Falls, } \\
\text { Minn. }\end{array}$ & $\begin{array}{l}\text { Integration } \\
\text { Pumped }\end{array}$ & $\begin{array}{l}121.1 \\
507\end{array}$ & $\begin{array}{l}101.7 \\
507\end{array}$ & $\begin{array}{l}101.7 \\
240\end{array}$ & $\begin{array}{l}2,35 \\
2,35\end{array}$ & $\begin{array}{l}5 / 16 \\
--\end{array}$ & $\begin{array}{c}5.0-80 \\
\ldots\end{array}$ & $\begin{array}{l}62 \\
96\end{array}$ \\
\hline $7-06$ & $\begin{array}{l}\text { Minnesota } R \text {. at } \\
\text { Mile 3.5, Minn. }\end{array}$ & $\begin{array}{l}\text { Integration } \\
\text { Pumped }\end{array}$ & $\begin{array}{l}122.8 \\
526\end{array}$ & $\begin{array}{l}101.9 \\
526\end{array}$ & $\begin{array}{l}101.9 \\
526\end{array}$ & $\begin{array}{l}2,35 \\
2,35\end{array}$ & $\begin{array}{c}1 / 4-5 / 16 \\
--\end{array}$ & $\begin{array}{c}7.0- \\
17.0 \\
--\end{array}$ & $\begin{array}{l}50 \\
84\end{array}$ \\
\hline $7-08$ & $\begin{array}{l}\text { Mississippi R. at } \\
\text { Hastings, Minn. }\end{array}$ & $\begin{array}{l}\text { Integration } \\
\text { Pumped }\end{array}$ & $\begin{array}{l}109.4 \\
511\end{array}$ & $\begin{array}{l}102.1 \\
511\end{array}$ & $\begin{array}{l}102.1 \\
511\end{array}$ & $\begin{array}{l}8 \\
8\end{array}$ & $\begin{array}{c}5 / 16 \\
--\end{array}$ & $\begin{array}{l}11 \\
--\end{array}$ & $\begin{array}{l}57 \\
87\end{array}$ \\
\hline $7-08$ & $\begin{array}{l}\text { St. Croix R. at } \\
\text { Mile } 0.5, \text { Wis. }\end{array}$ & Dipped & 42.9 & 0 & 41.6 & 1 & - & - & -- \\
\hline $7-10$ & $\begin{array}{l}\text { Mississippi R. near } \\
\text { Pepin, Wis. }\end{array}$ & $\begin{array}{l}\text { Integration } \\
\text { Pumped }\end{array}$ & $\begin{array}{l}121.7 \\
509\end{array}$ & $\begin{array}{l}112.8 \\
509\end{array}$ & $\begin{array}{l}112.8 \\
509\end{array}$ & $\begin{array}{l}{ }^{3} 12 \\
{ }^{3} 12\end{array}$ & $\begin{array}{c}5 / 16 \\
--\end{array}$ & $\begin{array}{c}2.0-8.5 \\
\ldots\end{array}$ & $\begin{array}{l}69 \\
98\end{array}$ \\
\hline $7-10$ & $\begin{array}{l}\text { Chippewa R, at } \\
\text { Mile } 1.7, \text { Wis. }\end{array}$ & Dipped & 38.9 & 0 & 37.6 & 1 & -- & -- & -- \\
\hline $7-12$ & $\begin{array}{l}\text { Mississippi R. at } \\
\text { Trempealeau, Wis. }\end{array}$ & $\begin{array}{l}\text { Integration } \\
\text { Pumped }\end{array}$ & $\begin{array}{l}126.3 \\
717\end{array}$ & $\begin{array}{l}117.8 \\
717\end{array}$ & $\begin{array}{l}117.8 \\
489\end{array}$ & $\begin{array}{l}8 \\
8\end{array}$ & $\begin{array}{c}5 / 16 \\
--\end{array}$ & $\begin{array}{l}7.0 \\
--\end{array}$ & $\begin{array}{l}61 \\
80\end{array}$ \\
\hline $7-15$ & $\begin{array}{l}\text { Mississippi R. below } \\
\text { Lock and Dam 9, Wis. }\end{array}$ & $\begin{array}{l}\text { Integration } \\
\text { Pumped }\end{array}$ & $\begin{array}{l}133.5 \\
603\end{array}$ & $\begin{array}{l}114.1 \\
603\end{array}$ & $\begin{array}{l}114.1 \\
603\end{array}$ & $\begin{array}{l}9 \\
9\end{array}$ & $\begin{array}{c}5 / 16 \\
--\end{array}$ & $\begin{array}{l}7.0 \\
--\end{array}$ & $\begin{array}{l}69 \\
88\end{array}$ \\
\hline $7-15$ & $\begin{array}{l}\text { Wisconsin R. at } \\
\text { Mile } \sim 1.0, \text { Wis. }\end{array}$ & Dipped & 38.6 & 0 & 37.3 & 1 & - & -- & -- \\
\hline $7-18$ & $\begin{array}{l}\text { Mississippi R. at } \\
\text { Clinton, lowa }\end{array}$ & $\begin{array}{l}\text { Integration } \\
\text { Pumped }\end{array}$ & $\begin{array}{l}126.5 \\
974\end{array}$ & $\begin{array}{l}103.8 \\
974\end{array}$ & $\begin{array}{l}103.8 \\
743\end{array}$ & $\begin{array}{l}9 \\
9\end{array}$ & $\begin{array}{c}5 / 16 \\
--\end{array}$ & $\begin{array}{c}8.0 \\
--\end{array}$ & $\begin{array}{l}48 \\
87\end{array}$ \\
\hline $7-20$ & $\begin{array}{l}\text { Rock R. at } \\
\quad \text { Mile } \sim 1.0, \text { Ill. }\end{array}$ & Dipped & 38.9 & 0 & 37.6 & 1 & -- & -- & - \\
\hline $7-20$ & $\begin{array}{l}\text { Iowa R. at } \\
\text { Mile } \sim 1.0, \text { Iowa }\end{array}$ & Dipped & 39.6 & 0 & 38.3 & 1 & -- & -- & -- \\
\hline $7-21$ & $\begin{array}{l}\text { Mississippi R. at } \\
\text { Keokuk, lowa }\end{array}$ & $\begin{array}{l}\text { Integration } \\
\text { Pumped }\end{array}$ & $\begin{array}{l}147.5 \\
1004\end{array}$ & $\begin{array}{l}115.8 \\
1004\end{array}$ & $\begin{array}{l}115.8 \\
763\end{array}$ & $\begin{array}{l}9 \\
9\end{array}$ & $\begin{array}{c}5 / 16 \\
-\end{array}$ & $\begin{array}{l}5.0 \\
--\end{array}$ & $\begin{array}{l}59 \\
86\end{array}$ \\
\hline $7-22$ & $\begin{array}{l}\text { Des Moines R. at } \\
\text { Mile } \sim 1.0 \text {, lowa }\end{array}$ & Dipped & 39.3 & 0 & 38.0 & 1 & -- & -- & -- \\
\hline $7-24$ & $\begin{array}{l}\text { Mississippi R. near } \\
\text { Winfield, Mo. }\end{array}$ & $\begin{array}{l}\text { Integration } \\
\text { Pumped }\end{array}$ & $\begin{array}{l}119.2 \\
507\end{array}$ & $\begin{array}{l}100.0 \\
507\end{array}$ & $\begin{array}{l}100.0 \\
507\end{array}$ & $\begin{array}{l}10 \\
10\end{array}$ & $\begin{array}{c}1 / 4 \\
--\end{array}$ & $\begin{array}{l}7.5 \\
--\end{array}$ & $\begin{array}{l}47 \\
85\end{array}$ \\
\hline $7-25$ & $\begin{array}{l}\text { Illinois R. at } \\
\text { Hardin, Ill. }\end{array}$ & $\begin{array}{l}\text { Integration } \\
\text { Pumped } \\
\text { Pumped }\end{array}$ & $\begin{array}{l}30.7 \\
104 \\
854\end{array}$ & $\begin{array}{l}0 \\
104 \\
854\end{array}$ & $\begin{array}{l}0 \\
104 \\
649\end{array}$ & $\begin{array}{l}21 \\
21 \\
21\end{array}$ & $\begin{array}{c}\text { None }^{4} \\
-- \\
--\end{array}$ & $\begin{array}{c}2.0 \\
-- \\
--\end{array}$ & $\begin{array}{r}-- \\
81 \\
101\end{array}$ \\
\hline $7-27$ & $\begin{array}{l}\text { Missouri R. at } \\
\text { St. Charles, Mo. }\end{array}$ & $\begin{array}{l}\text { Integration } \\
\text { Pumped }\end{array}$ & $\begin{array}{l}118.5 \\
508\end{array}$ & $\begin{array}{l}94.8 \\
508\end{array}$ & $\begin{array}{l}94.8 \\
508\end{array}$ & $\begin{array}{l}15 \\
15\end{array}$ & $\begin{array}{l}1 / 4 \\
--\end{array}$ & $\begin{array}{l}5.0 \\
--\end{array}$ & $\begin{array}{l}49 \\
93\end{array}$ \\
\hline
\end{tabular}


Table 10.--Volumes of river water collected and processed, assoclated sampling parameters, and percent recovery of suspended sediment for the July-August 1991 cruise--Continued

\begin{tabular}{|c|c|c|c|c|c|c|c|c|c|}
\hline \multirow[b]{2}{*}{$\begin{array}{l}\text { Date } \\
1991\end{array}$} & \multirow[b]{2}{*}{ Site name } & \multirow[b]{2}{*}{$\begin{array}{l}\text { Collecting } \\
\text { method }\end{array}$} & \multicolumn{3}{|c|}{ Sample volumes } & \multicolumn{3}{|c|}{ Sampling parameters } & \multirow{2}{*}{$\begin{array}{c}\text { Reco very } \\
\text { of } \\
\text { suspended } \\
\text { sediment } \\
\text { less than } \\
63 \text { fim' } \\
\text { ( } \% \text { i) }\end{array}$} \\
\hline & & & $\begin{array}{l}V_{c} \\
(L)\end{array}$ & $\begin{array}{l}\mathbf{V}_{\mathrm{d}} \\
(\mathrm{L})\end{array}$ & $\begin{array}{l}v_{u} \\
(L)\end{array}$ & $\begin{array}{l}\text { Num- } \\
\text { ber } \\
\text { of } \\
\text { vertl- } \\
\text { cals }\end{array}$ & $\begin{array}{c}\text { Nozzle } \\
\text { size } \\
\text { (Inches) }\end{array}$ & $\begin{array}{c}\text { Transit } \\
\text { rate } \\
\text { (cm/s) }\end{array}$ & \\
\hline $7-28$ & $\begin{array}{l}\text { Kaskaskia R. at } \\
\text { Mile 1.5, III. }\end{array}$ & Dipped & 39.9 & 0 & 38.7 & 1 & -- & -- & -- \\
\hline $7-29$ & $\begin{array}{l}\text { Mississippi R. at } \\
\text { Thebes, Ill. }\end{array}$ & $\begin{array}{l}\text { Integration } \\
\text { Pumped }\end{array}$ & $\begin{array}{l}115.6 \\
611\end{array}$ & $\begin{array}{l}96.5 \\
611\end{array}$ & $\begin{array}{l}96.5 \\
608\end{array}$ & $\begin{array}{l}15 \\
15\end{array}$ & $\begin{array}{l}T / 4 \\
--\end{array}$ & $\begin{array}{c}10.0 \\
--\end{array}$ & $\begin{array}{l}48 \\
88\end{array}$ \\
\hline $7-30$ & $\begin{array}{l}\text { Ohio R. at } \\
\quad \text { Olmsted, IIl. }\end{array}$ & $\begin{array}{l}\text { Pumped } \\
\text { Pumped }\end{array}$ & $\begin{array}{l}121.0 \\
1006\end{array}$ & $\begin{array}{l}103.9 \\
1006\end{array}$ & $\begin{array}{l}103.9 \\
1001\end{array}$ & $\begin{array}{l}15 \\
15\end{array}$ & -- & - & $\begin{array}{l}61 \\
77\end{array}$ \\
\hline $8-01$ & $\begin{array}{l}\text { White R. at } \\
\text { Mile I.2, Ark. }\end{array}$ & Dipped & 42.6 & 0 & 38.6 & 1 & -- & -- & -- \\
\hline $8-01$ & $\begin{array}{l}\text { Arkansas R. at } \\
\text { Mile 0.0, Ark. }\end{array}$ & Dipped & 42.6 & 0 & 38.6 & 1 & -- & - & $\cdots$ \\
\hline $8-02$ & $\begin{array}{l}\text { Yazoo R, at } \\
\text { Mile } \sim 3.0, \text { Miss. }\end{array}$ & Dipped & 42.0 & 0 & 38.0 & I & -- & -- & - \\
\hline $8-03$ & $\begin{array}{l}\text { Mississippi R. below } \\
\text { Vicksburg, Miss. }\end{array}$ & $\begin{array}{l}\text { Integration } \\
\text { Pumped }\end{array}$ & $\begin{array}{l}133.7 \\
621\end{array}$ & $\begin{array}{l}109.0 \\
621\end{array}$ & $\begin{array}{l}109.0 \\
621\end{array}$ & $\begin{array}{l}17 \\
17\end{array}$ & $\begin{array}{c}3 / 16 \\
--\end{array}$ & $\begin{array}{l}7.0 \\
--\end{array}$ & $\begin{array}{l}57 \\
90\end{array}$ \\
\hline $8-05$ & $\begin{array}{l}\text { Mississippi R. near } \\
\text { St. Francisville, La. }\end{array}$ & $\begin{array}{l}\text { Integration } \\
\text { Pumped }\end{array}$ & $\begin{array}{l}95.7 \\
603\end{array}$ & $\begin{array}{l}82.2 \\
603\end{array}$ & $\begin{array}{l}82.2 \\
603\end{array}$ & $\begin{array}{l}15 \\
15\end{array}$ & $\begin{array}{c}3 / 16 \\
-\cdot\end{array}$ & $\begin{array}{c}6.5 \\
--\end{array}$ & $\begin{array}{l}64 \\
85\end{array}$ \\
\hline $8-07$ & $\begin{array}{c}\text { Mississippi R. below } \\
\text { Belle Chasse, La. }\end{array}$ & $\begin{array}{l}\text { Pumped } \\
\text { Pumped }\end{array}$ & $\begin{array}{l}119.9 \\
1021\end{array}$ & $\begin{array}{l}113.5 \\
1021\end{array}$ & $\begin{array}{l}113.5 \\
1021\end{array}$ & $\begin{array}{l}5 \\
5\end{array}$ & $\begin{array}{l}-- \\
--\end{array}$ & - & $\begin{array}{l}6 \% \\
8 ?\end{array}$ \\
\hline
\end{tabular}

${ }^{1}$ See table 13 for total suspended-sediment concentration of silt and clay $(<63 \mu \mathrm{m})$ which was dried at $105-110^{\circ} \mathrm{C}$. Data for the integration and small volume pumped samples were provided by John Garbarino. They do not include the sediment trapped in the centrifuge bowl bottom sealirg unit; the silt and clay and colloid material were freeze dried. Data for the large volume pumped samples were provided by Colleen Rostad and do include the trapped sediment. The silt and clay were air dried at room temperature (about $23^{\circ} \mathrm{C}$ ) and the colloid material was freeze dried.

${ }^{2}$ Ship anchored at all verticals.

${ }^{3}$ Equal-discharge increments.

${ }^{4}$ The sampling bottle had a cap without the isokinetic nozzle; the intake diameter of the threaded hole in the cap was $1 / 2$ inch. 
Tabie 11.-Volumes of rlver water collected and processed, associated sampling parameters, and percent recovery of suspended sediment for the October-November 19?1 crulse

$\left[V_{c}\right.$, total volume collected; $V_{d}$, volume centrifuged; $V_{u}$, volume ultrafiltered; $L$, liters; $\mathrm{cm} / \mathrm{s}$, centimeters per second; $\mu \mathrm{m}$, micrometer; $\%$, percent; Integration, depth-integration; Pumped, discharge-weighted pumping from fixed depths; Dipped, surface dip sample at one location; --, dashes mean does not apply]

\begin{tabular}{|c|c|c|c|c|c|c|c|c|c|}
\hline \multirow[b]{2}{*}{$\begin{array}{l}\text { Date } \\
1991\end{array}$} & \multirow[b]{2}{*}{ Slte name } & \multirow[b]{2}{*}{$\begin{array}{l}\text { Collecting } \\
\text { method }\end{array}$} & \multicolumn{3}{|c|}{ Sample volumes } & \multicolumn{3}{|c|}{ Sampling parameters } & \multirow{2}{*}{$\begin{array}{l}\text { Recovery of } \\
\text { suspended } \\
\text { sediment } \\
\text { less than } \\
63 \mu \mathrm{m}^{1} \\
(\%)\end{array}$} \\
\hline & & & $\begin{array}{l}V_{c} \\
(L)\end{array}$ & $\begin{array}{l}V_{d} \\
(L)\end{array}$ & $\begin{array}{l}V_{u} \\
(L)\end{array}$ & $\begin{array}{l}\text { Num- } \\
\text { ber of } \\
\text { vertl- } \\
\text { cals }\end{array}$ & $\begin{array}{c}\text { Nozzle } \\
\text { slze } \\
\text { (Inches) }\end{array}$ & $\begin{array}{c}\text { Transit } \\
\text { rate } \\
\text { (cm/s) }\end{array}$ & \\
\hline $10-07$ & $\begin{array}{l}\text { Mississippi R. above } \\
\text { St. Anthony Falls, Minn. }\end{array}$ & $\begin{array}{l}\text { Integration } \\
\text { Pumped } \\
\text { Pumped }\end{array}$ & $\begin{array}{l}25.6 \\
120.0 \\
620\end{array}$ & $\begin{array}{r}0 \\
117 \\
620\end{array}$ & $\begin{array}{r}0 \\
117 \\
620\end{array}$ & $\begin{array}{l}25 \\
25 \\
25\end{array}$ & $\begin{array}{c}5 / 16 \\
-- \\
--\end{array}$ & $\begin{array}{c}2.0 \\
-- \\
--\end{array}$ & $\begin{array}{l}-- \\
54 \\
90\end{array}$ \\
\hline $10-08$ & $\begin{array}{l}\text { Minnesota R. at } \\
\text { Mile 3.5, Minn. }\end{array}$ & $\begin{array}{l}\text { Integration } \\
\text { Pumped } \\
\text { Pumped }\end{array}$ & $\begin{array}{l}12.6 \\
120.1 \\
759\end{array}$ & $\begin{array}{r}0 \\
117 \\
759\end{array}$ & $\begin{array}{r}0 \\
117 \\
511\end{array}$ & $\begin{array}{l}25 \\
25 \\
25\end{array}$ & $\begin{array}{c}5 / 16 \\
-- \\
--\end{array}$ & $\begin{array}{c}2.0 \\
-- \\
--\end{array}$ & $\begin{array}{c}-- \\
72 \\
94\end{array}$ \\
\hline $10-10$ & $\begin{array}{l}\text { Mississippi R. at } \\
\text { Hastings, Minn. }\end{array}$ & $\begin{array}{l}\text { Pumped } \\
\text { Pumped }\end{array}$ & $\begin{array}{l}120.0 \\
769\end{array}$ & $\begin{array}{l}114 \\
769\end{array}$ & $\begin{array}{l}114 \\
514\end{array}$ & $\begin{array}{l}3_{5} \\
3_{5}\end{array}$ & -- & -- & $\begin{array}{r}23 \\
102\end{array}$ \\
\hline $10-10$ & $\begin{array}{l}\text { St. Croix R. at } \\
\text { Mile } 0.5, W \text { is. }\end{array}$ & Dipped & 42.8 & 0 & 40.7 & 1 & -- & -- & -- \\
\hline $10-13$ & $\begin{array}{l}\text { Mississippi R. near } \\
\text { Pepin, Wis. }\end{array}$ & $\begin{array}{l}\text { Pumped } \\
\text { Pumped }\end{array}$ & $\begin{array}{l}120.0 \\
1201\end{array}$ & $\begin{array}{r}114 \\
1201\end{array}$ & $\begin{array}{l}114 \\
594\end{array}$ & $\begin{array}{l}6 \\
6\end{array}$ & -- & -- & $\begin{array}{r}{ }^{4} 161 \\
82\end{array}$ \\
\hline $10-12$ & $\begin{array}{l}\text { Chippewa R. at } \\
\text { Mile 1.7, Wis. }\end{array}$ & Dipped & 42.2 & 0 & 38.0 & 1 & - & -- & -- \\
\hline $10-15$ & $\begin{array}{l}\text { Mississippi R. at } \\
\text { Trempealeau, Wis. }\end{array}$ & $\begin{array}{l}\text { Pumped } \\
\text { Pumped }\end{array}$ & $\begin{array}{l}120.1 \\
1009\end{array}$ & $\begin{array}{r}114 \\
1009\end{array}$ & $\begin{array}{l}114 \\
499\end{array}$ & $\begin{array}{l}7 \\
7\end{array}$ & - & -- & $\begin{array}{l}12 \\
87\end{array}$ \\
\hline $10-18$ & $\begin{array}{l}\text { Mississippi R. below } \\
\text { Lock and Dam 9, Wis. }\end{array}$ & $\begin{array}{l}\text { Pumped } \\
\text { Pumped }\end{array}$ & $\begin{array}{l}120.0 \\
1017\end{array}$ & $\begin{array}{r}114 \\
1017\end{array}$ & $\begin{array}{l}114 \\
757\end{array}$ & $\begin{array}{l}6 \\
6\end{array}$ & - & -- & $\begin{array}{l}65 \\
89\end{array}$ \\
\hline $10-19$ & $\begin{array}{l}\text { Wisconsin R. at } \\
\text { Mile } \sim 1.0, \text { Wis. }\end{array}$ & Dipped & 42 & 0 & -38 & 1 & - & -- & -- \\
\hline $10-22$ & $\begin{array}{r}\text { Mississippi R. at } \\
\text { Clinton, Jowa }\end{array}$ & $\begin{array}{l}\text { Pumped } \\
\text { Pumped }\end{array}$ & $\begin{array}{l}120.1 \\
770\end{array}$ & $\begin{array}{l}114 \\
770\end{array}$ & $\begin{array}{l}114 \\
531\end{array}$ & $\begin{array}{l}6 \\
6\end{array}$ & -- & $\cdots$ & $\begin{array}{l}65 \\
90\end{array}$ \\
\hline $10-24$ & $\begin{array}{l}\text { Rock R, at } \\
\text { Mile } \sim 1,0,111 .\end{array}$ & Dipped & 42.2 & 0 & 38.4 & 1 & -- & -- & -- \\
\hline $10-25$ & $\begin{array}{l}\text { lowa } R \text {. at } \\
\quad \text { Mile } \sim 1.0, \text { lowa }\end{array}$ & Dipped & 42.0 & 0 & 38.2 & 1 & -- & -- & -- \\
\hline $10-27$ & $\begin{array}{l}\text { Mississippi R. at } \\
\text { Keokuk, lowa }\end{array}$ & $\begin{array}{l}\text { Pumped } \\
\text { Pumped }\end{array}$ & $\begin{array}{l}120.0 \\
1011\end{array}$ & $\begin{array}{r}114 \\
1011\end{array}$ & $\begin{array}{l}114 \\
505\end{array}$ & $\begin{array}{l}7 \\
7\end{array}$ & -- & - & $\begin{array}{l}81 \\
92\end{array}$ \\
\hline $10-28$ & $\begin{array}{l}\text { Des Moines, R. at } \\
\text { Mile } \sim 1.0, \text { lowa }\end{array}$ & Dipped & 42.0 & 0 & 38.2 & 1 & -- & - & - \\
\hline $10-30$ & $\begin{array}{l}\text { Mississippi R. near } \\
\text { Winfield, Mo. }\end{array}$ & $\begin{array}{l}\text { Pumped } \\
\text { Pumped }\end{array}$ & $\begin{array}{l}120.0 \\
505\end{array}$ & $\begin{array}{l}114 \\
505\end{array}$ & $\begin{array}{l}114 \\
505\end{array}$ & $\begin{array}{l}7 \\
7\end{array}$ & $\begin{array}{l}-- \\
--\end{array}$ & -- & $\begin{array}{l}72 \\
98\end{array}$ \\
\hline $10-31$ & $\begin{array}{l}\text { Illinois R. at } \\
\text { Hardin, III. }\end{array}$ & $\begin{array}{l}\text { Pumped } \\
\text { Pumped }\end{array}$ & $\begin{array}{l}120.0 \\
1000\end{array}$ & $\begin{array}{r}114 \\
1000\end{array}$ & $\begin{array}{r}114 \\
500\end{array}$ & $\begin{array}{l}21 \\
21\end{array}$ & -- & -- & $\begin{array}{r}83 \\
111\end{array}$ \\
\hline $11-03$ & $\begin{array}{l}\text { Missouri R. at } \\
\text { St. Charles, Mo. }\end{array}$ & $\begin{array}{l}\text { Integration } \\
\text { Pumped }\end{array}$ & $\begin{array}{l}94.3 \\
498\end{array}$ & $\begin{array}{l}76.8 \\
498\end{array}$ & $\begin{array}{l}76.8 \\
498\end{array}$ & $\begin{array}{l}10 \\
10\end{array}$ & $\begin{array}{l}1 / 4 \\
--\end{array}$ & $\begin{array}{c}5.0 \\
--\end{array}$ & $\begin{array}{l}50 \\
89\end{array}$ \\
\hline
\end{tabular}


Table 11.--Volumes of river water collected and processed, associated sampling parameters, and percent recovery of suspended sediment for the October-November 1991 cruise-

\section{Continued}

\begin{tabular}{|c|c|c|c|c|c|c|c|c|c|}
\hline \multirow[b]{2}{*}{$\begin{array}{l}\text { Date } \\
1991\end{array}$} & \multirow[b]{2}{*}{ Site name } & \multirow[b]{2}{*}{$\begin{array}{l}\text { Collecting } \\
\text { method }\end{array}$} & \multicolumn{3}{|c|}{ Sample volumes } & \multicolumn{3}{|c|}{ Sampling parameters } & \multirow{2}{*}{$\begin{array}{l}\text { Recovery of } \\
\text { susprnded } \\
\text { sediment } \\
\text { less than } \\
63 \mu \mathrm{m}^{1} \\
(9 ; \%)\end{array}$} \\
\hline & & & $\begin{array}{l}V_{c} \\
(L)\end{array}$ & $\begin{array}{l}\mathbf{V}_{d} \\
(L)\end{array}$ & $\begin{array}{l}V_{u} \\
(L)\end{array}$ & $\begin{array}{l}\text { Num- } \\
\text { ber of } \\
\text { verti- } \\
\text { cals }\end{array}$ & $\begin{array}{c}\text { Nozzie } \\
\text { slze } \\
\text { (Inches) }\end{array}$ & $\begin{array}{c}\text { Transit } \\
\text { rate } \\
\text { (cm/s) }\end{array}$ & \\
\hline$\overline{11-04}$ & $\begin{array}{c}\text { Kaskaskia R. at } \\
\text { Mile 1.5, } 111 .\end{array}$ & Dipped & $\sim 42$ & 0 & 40.5 & $T$ & - & - & $\cdots$ \\
\hline $11-05$ & $\begin{array}{l}\text { Mississippi R. at } \\
\text { Thebes, Ill. }\end{array}$ & $\begin{array}{l}\text { Integration } \\
\text { Pumped }\end{array}$ & $\begin{array}{l}83.7 \\
501\end{array}$ & $\begin{array}{l}66.2 \\
501\end{array}$ & $\begin{array}{l}66.2 \\
501\end{array}$ & $\begin{array}{l}12 \\
12\end{array}$ & $\begin{array}{l}1 / 4 \\
--\end{array}$ & $\begin{array}{c}10.0 \\
--\end{array}$ & $\begin{array}{l}35 \\
91\end{array}$ \\
\hline $11-06$ & $\begin{array}{l}\text { Ohio R. at } \\
\text { Olmsted, III. }\end{array}$ & $\begin{array}{l}\text { Pumped } \\
\text { Pumped }\end{array}$ & $\begin{array}{l}120.0 \\
996\end{array}$ & $\begin{array}{l}114 \\
996\end{array}$ & $\begin{array}{l}114 \\
500\end{array}$ & $\begin{array}{l}11 \\
11\end{array}$ & -- & -- & $\begin{array}{l}3 ? \\
81\end{array}$ \\
\hline $11-08$ & $\begin{array}{l}\text { White R. at } \\
\text { Mile 1.2, Ark. }\end{array}$ & Dipped & 41.1 & 0 & 37.6 & 1 & -- & -. & -- \\
\hline $11-08$ & $\begin{array}{l}\text { Arkansas R. at } \\
\text { Mile 0.0, Ark. }\end{array}$ & Dipped & 41.0 & 0 & 37.5 & 1 & -- & $\cdots$ & -- \\
\hline $11-10$ & $\begin{array}{l}\text { Yazoo R. at } \\
\text { Mile } ~ 3.0, \text { Miss. }\end{array}$ & Dipped & 39.8 & 0 & 36.3 & 1 & - & -- & - \\
\hline $11-09$ & $\begin{array}{l}\text { Mississippi R. below } \\
\text { Vicksburg, Miss. }\end{array}$ & $\begin{array}{l}\text { Integration } \\
\text { Pumped }\end{array}$ & $\begin{array}{l}82.0 \\
503\end{array}$ & $\begin{array}{l}64.5 \\
503\end{array}$ & $\begin{array}{l}64.5 \\
503\end{array}$ & $\begin{array}{l}13 \\
13\end{array}$ & $\begin{array}{c}3 / 16 \\
--\end{array}$ & $\begin{array}{c}9.5 \\
--\end{array}$ & 3) \\
\hline $11-11$ & $\begin{array}{l}\text { Mississippi R. near } \\
\text { St. Francisville, La. }\end{array}$ & $\begin{array}{l}\text { Integration } \\
\text { Pumped }\end{array}$ & $\begin{array}{l}83.4 \\
501\end{array}$ & $\begin{array}{l}66.9 \\
501\end{array}$ & $\begin{array}{l}66.9 \\
501\end{array}$ & $\begin{array}{l}12 \\
12\end{array}$ & $\begin{array}{c}3 / 16 \\
--\end{array}$ & $\begin{array}{l}7.5 \\
--\end{array}$ & $\begin{array}{l}51) \\
85\end{array}$ \\
\hline $11-13$ & $\begin{array}{c}\text { Mississippi R. below } \\
\text { Belle Chasse, La. }\end{array}$ & $\begin{array}{l}\text { Integration } \\
\text { Pumped } \\
\text { Pumped }\end{array}$ & $\begin{array}{r}32.1 \\
120.0 \\
1000\end{array}$ & $\begin{array}{r}0 \\
114 \\
1000\end{array}$ & $\begin{array}{r}0 \\
114 \\
500\end{array}$ & $\begin{array}{l}5 \\
5 \\
5\end{array}$ & $\begin{array}{l}1 / 4 \\
-- \\
--\end{array}$ & $\begin{array}{c}6.0 \\
-- \\
--\end{array}$ & $\begin{array}{l}- \\
67 \\
8 \vdots\end{array}$ \\
\hline
\end{tabular}

\footnotetext{
${ }^{1}$ See table 14 for total suspended-sediment concentration of silt and clay $(<63 \mu \mathrm{m})$ which was dried at $105-110^{\circ} \mathrm{C}$. Data for the integration and small volume pumped samples were provided by John Garbarino. They do not include the sediment trapped in the centrifuge bowl bottom sealing unit; the silt and clay and colloid material were freeze dried. Data for the large volume pumped samples were provided by Colleen Rostad and do include the trapped sediment. The silt and clay were air dried at room temperature (about $23^{\circ} \mathrm{C}$ ) and the colloid material was freeze dried.

${ }^{2}$ Ship anchored at all verticals.

${ }^{3}$ Ship anchored at two verticals.

${ }^{4}$ Data associated with this sample are questionable.
} 
Tabie 12.--Volumes of river water collected and processed, assoclated sampilng parameters, and percent recovery of suspended sediment for the Aprll-May $1992 \mathrm{cr}$.lise

$\left[\mathrm{V}_{\mathrm{c}}\right.$, total volume collected; $\mathrm{V}_{\mathrm{d}}$, volume centrifuged; $\mathrm{V}_{\mathrm{u}}$, volume ultrafiltered; $\mathrm{L}, \mathrm{l}^{\text {iters; }} \mathrm{cm}$ /

$s$, centimeters per second; $\mu \mathrm{m}$, micrometer; \%, percent; Integration, depth-interration;

Pumped, discharge-weighted pumping from fixed depths; Dipped, surface dip sample at one location; --, does not apply]

\begin{tabular}{|c|c|c|c|c|c|c|c|c|c|}
\hline \multirow[b]{2}{*}{$\begin{array}{l}\text { Date } \\
1992\end{array}$} & \multirow[b]{2}{*}{ Site name } & \multirow[b]{2}{*}{$\begin{array}{l}\text { Collecting } \\
\text { method }\end{array}$} & \multicolumn{3}{|c|}{ Sample volumes } & \multicolumn{3}{|c|}{ Sampllng parameters } & \multirow{2}{*}{$\begin{array}{l}\text { Recovery of } \\
\text { suspended } \\
\text { sediment less } \\
\text { than } \\
63 \mu \mathrm{m}^{1} \\
(\%)\end{array}$} \\
\hline & & & $\begin{array}{l}V_{c} \\
\text { (L) }\end{array}$ & $\begin{array}{l}V_{d} \\
(L)\end{array}$ & $\begin{array}{l}v_{u} \\
(L)\end{array}$ & $\begin{array}{l}\text { ber of } \\
\text { vertt- } \\
\text { cals }\end{array}$ & $\begin{array}{l}\text { Nozzle slze } \\
\text { (Inches) }\end{array}$ & $\begin{array}{l}\text { Translt } \\
\text { rate } \\
(\mathrm{cm} / \mathrm{s})\end{array}$ & \\
\hline $4-06$ & $\begin{array}{l}\text { Mississippi R. above } \\
\text { St. Anthony Falls, } \\
\text { Minn. }\end{array}$ & $\begin{array}{l}\text { Integration } \\
\text { Pumped }\end{array}$ & $\begin{array}{l}98.6 \\
758\end{array}$ & $\begin{array}{l}92.4 \\
758\end{array}$ & $\begin{array}{l}92.4 \\
758\end{array}$ & $\begin{array}{l}2,35 \\
2,35\end{array}$ & $\begin{array}{c}5 / 16 \\
--\end{array}$ & $\begin{array}{c}1.0-4.0 \\
--\end{array}$ & $\begin{array}{l}50 \\
72\end{array}$ \\
\hline $4-08$ & $\begin{array}{l}\text { Minnesota R. at } \\
\text { Mile 3.5, Minn. }\end{array}$ & $\begin{array}{l}\text { Integration } \\
\text { Pumped } \\
\text { Pumped }\end{array}$ & $\begin{array}{l}75.1 \\
11.9 \\
773\end{array}$ & $\begin{array}{c}56.5 \\
0 \\
773\end{array}$ & $\begin{array}{c}56.5 \\
0 \\
773\end{array}$ & $\begin{array}{l}2,34 \\
2,34 \\
2,34\end{array}$ & $\begin{array}{c}5 / 16 \\
- \\
--\end{array}$ & $\begin{array}{c}2.5-6.5 \\
- \\
-\end{array}$ & $\begin{array}{c}69 \\
\cdots \\
97\end{array}$ \\
\hline $4-10$ & $\begin{array}{l}\text { Mississippi R. at } \\
\text { Hastings, Minn. }\end{array}$ & $\begin{array}{l}\text { Integration } \\
\text { Pumped } \\
\text { Pumped } \\
\text { Pumped }\end{array}$ & $\begin{array}{r}19.7 \\
100.1 \\
11.9 \\
804\end{array}$ & $\begin{array}{c}0 \\
98.9 \\
0 \\
804\end{array}$ & $\begin{array}{c}0 \\
98.9 \\
0 \\
420\end{array}$ & $\begin{array}{l}7 \\
7 \\
7 \\
7\end{array}$ & $\begin{array}{c}5 / 16 \\
- \\
- \\
-\end{array}$ & $\begin{array}{c}3.0-8.0 \\
- \\
-- \\
-\end{array}$ & $\begin{array}{r}-- \\
72 \\
- \\
95\end{array}$ \\
\hline $4-11$ & $\begin{array}{l}\text { St. Croix R. at } \\
\text { Mile 0.5, Wis. }\end{array}$ & Dipped & 43.2 & 0 & $\begin{array}{l}\text { Unmea- } \\
\text { sured }\end{array}$ & 1 & -- & - & - \\
\hline $4-12$ & $\begin{array}{l}\text { Mississippi R. near } \\
\text { Pepin, Wis. }\end{array}$ & $\begin{array}{l}\text { Pumped } \\
\text { Pumped }\end{array}$ & $\begin{array}{c}155.2 \\
1002\end{array}$ & $\begin{array}{c}149.3 \\
1002\end{array}$ & $\begin{array}{l}149.3 \\
413\end{array}$ & $\begin{array}{l}7 \\
7\end{array}$ & - & - & $\begin{array}{l}75 \\
94\end{array}$ \\
\hline 4-12 & $\begin{array}{l}\text { Chippewa R. at } \\
\text { Mile 1.7, Wis. }\end{array}$ & Dipped & 40.3 & 0 & 35.9 & 1 & -- & - & - \\
\hline 4-14 & $\begin{array}{l}\text { Mississippi R. at } \\
\text { Trempealeau, Wis. }\end{array}$ & $\begin{array}{l}\text { Integration } \\
\text { Pumped }\end{array}$ & $\begin{array}{c}112.4 \\
1001\end{array}$ & $\begin{array}{c}105.2 \\
1001\end{array}$ & $\begin{array}{l}105.2 \\
506\end{array}$ & $\begin{array}{l}7 \\
7\end{array}$ & $\begin{array}{c}5 / 16 \\
--\end{array}$ & $\begin{array}{c}5.5 \\
\cdots\end{array}$ & $\begin{array}{l}56 \\
96\end{array}$ \\
\hline $4-17$ & $\begin{array}{l}\text { Mississippi R. below } \\
\text { Lock and Dam 9, } \\
\text { Wis. }\end{array}$ & $\begin{array}{l}\text { Integration } \\
\text { Pumped }\end{array}$ & $\begin{array}{l}118.4 \\
752\end{array}$ & $\begin{array}{l}88.4 \\
752\end{array}$ & $\begin{array}{l}88.4 \\
503\end{array}$ & $\begin{array}{l}7 \\
7\end{array}$ & $\begin{array}{c}5 / 16 \\
-\end{array}$ & $\begin{array}{c}6.0 \\
--\end{array}$ & $\begin{array}{l}55 \\
94\end{array}$ \\
\hline $4-17$ & $\begin{array}{l}\text { Wisconsin R at } \\
\quad \text { Mile } \sim 1.0, \text { Wis. }\end{array}$ & Dipped & 41.6 & 0 & $\begin{array}{l}\text { Unmea- } \\
\text { sured }\end{array}$ & 1 & - & - & -- \\
\hline $4-18$ & $\begin{array}{r}\text { Mississippi R. at } \\
\text { Clinton, Iowa }\end{array}$ & $\begin{array}{l}\text { Integration } \\
\text { Pumped } \\
\text { Pumped }\end{array}$ & $\begin{array}{c}116.4 \\
12.6 \\
605\end{array}$ & $\begin{array}{c}98.7 \\
0 \\
605\end{array}$ & $\begin{array}{c}98.7 \\
0 \\
500\end{array}$ & $\begin{array}{l}7 \\
7 \\
7\end{array}$ & $\begin{array}{c}1 / 4 \\
-- \\
--\end{array}$ & $\begin{array}{c}6.0 \\
- \\
-\end{array}$ & $\begin{array}{r}74 \\
-- \\
87\end{array}$ \\
\hline $4-20$ & $\begin{array}{l}\text { Rock R. at } \\
\qquad \text { Mile } \sim 1.0,111 .\end{array}$ & Dipped & 41.7 & 0 & $\begin{array}{l}\text { Unmea- } \\
\text { sured }\end{array}$ & 1 & -- & -- & -- \\
\hline $4-22$ & $\begin{array}{l}\text { Jowa } R \text {. at } \\
\quad \text { Mile } \sim 1.0, \text { lowa }\end{array}$ & Dipped & 40.3 & 0 & $\begin{array}{l}\text { Unmea- } \\
\text { sured }\end{array}$ & 1 & - & - & - \\
\hline $4-23$ & $\begin{array}{r}\text { Mississippi R. at } \\
\text { Keokuk, lowa }\end{array}$ & $\begin{array}{l}\text { Integration } \\
\text { Pumped } \\
\text { Pumped }\end{array}$ & $\begin{array}{c}67.4 \\
12.1 \\
1011\end{array}$ & $\begin{array}{r}50 . I \\
0 \\
1011\end{array}$ & $\begin{array}{c}50.1 \\
0 \\
505\end{array}$ & $\begin{array}{l}7 \\
7 \\
7\end{array}$ & $\begin{array}{c}5 / 16 \\
-- \\
-\end{array}$ & $\begin{array}{l}8.5 \\
-- \\
--\end{array}$ & $\begin{array}{c}82 \\
-- \\
50\end{array}$ \\
\hline $4-24$ & $\begin{array}{l}\text { Des Moines } R \text {. at } \\
\text { Mile } \sim 1.0 \text {, lowa }\end{array}$ & Dipped & 40.3 & 0 & $\begin{array}{l}\text { Unmea- } \\
\text { sured }\end{array}$ & 1 & -- & -- & -- \\
\hline
\end{tabular}


Table 12.--Volumes of river water collected and processed, associated sampling paramet $: r s$, and percent recovery of suspended sediment for the April-May 1992 cruise-Continuert

\begin{tabular}{|c|c|c|c|c|c|c|c|c|c|}
\hline \multirow[b]{2}{*}{$\begin{array}{l}\text { Date } \\
1992\end{array}$} & \multirow[b]{2}{*}{ Site name } & \multirow[b]{2}{*}{$\begin{array}{l}\text { Coliecting } \\
\text { method }\end{array}$} & \multicolumn{3}{|c|}{ Sampie voiumes } & \multicolumn{3}{|c|}{ Sampllng parameters } & \multirow{2}{*}{$\begin{array}{l}\text { Recovory of } \\
\text { suspe } \\
\text { sediment less } \\
\text { than } \\
63 \mu^{1} \\
\text { (\%) }\end{array}$} \\
\hline & & & $\begin{array}{l}\mathbf{V}_{\mathrm{c}} \\
(\mathrm{L})\end{array}$ & $\begin{array}{l}v_{d} \\
(L)\end{array}$ & $\begin{array}{l}v_{u} \\
(L)\end{array}$ & $\begin{array}{l}\text { Num- } \\
\text { ber of } \\
\text { vertl- } \\
\text { cals }\end{array}$ & $\begin{array}{c}\text { Nozzle size } \\
\text { (inches) }\end{array}$ & $\begin{array}{c}\text { Translt } \\
\text { rate } \\
(\mathrm{cm} / \mathrm{s})\end{array}$ & \\
\hline \multirow[t]{3}{*}{$4-26$} & Mississippi R. near & Integration & 83.4 & 66.3 & 66.3 & 10 & $1 / 4$ & 11.0 & 59 \\
\hline & Winfield, Mo. & Pumped & 12.0 & 0 & 0 & 10 & -- & -- & - \\
\hline & & Pumped & 503 & 503 & 503 & 10 & -- & -- & 73 \\
\hline \multirow[t]{3}{*}{$4-27$} & Illinois R. at & Integration & 92.4 & 75.0 & 75.0 & 5 & $5 / 16$ & 5.0 & 82 \\
\hline & Hardin, 111. & Pumped & 12.1 & 0 & 0 & 5 & -- & -- & - \\
\hline & & Pumped & 500 & 500 & 500 & 5 & -- & -- & 115 \\
\hline \multirow[t]{3}{*}{$4-29$} & Missouri R. at & Integration & 48.1 & 38.7 & 38.7 & 8 & $1 / 4,3 / 16$ & $8.0,14.0$ & 61 \\
\hline & St. Charles, Mo. & Pumped & 12.1 & 0 & 0 & 8 & -- & -- & -- \\
\hline & & Pumped & 259 & 259 & 259 & 8 & - & -- & 100 \\
\hline $4-30$ & $\begin{array}{c}\text { Kaskaskia R. at } \\
\text { Mile 1.5, III. }\end{array}$ & Dipped & 41.0 & 0 & 37.7 & 1 & -- & -- & -- \\
\hline \multirow[t]{3}{*}{$5-01$} & Mississippi R. at & Integration & 69.3 & 56.5 & 56.5 & 9 & $3 / 16$ & 15.0 & 53 \\
\hline & Thebes, Ill. & Pumped & 12.2 & 0 & 0 & 9 & -- & - & - \\
\hline & & Pumped & 501 & 501 & 501 & 9 & -- & - & 99 \\
\hline \multirow[t]{3}{*}{$5-02$} & Ohio R. at & Pumped & 20.0 & 0 & 0 & II & - & - & - \\
\hline & Olmsted, III. & Pumped & 120.1 & 114.2 & 114.2 & 11 & -- & - & 64 \\
\hline & & Pumped & 1001 & 1001 & 991 & 11 & - & - & 94 \\
\hline $5-05$ & $\begin{array}{l}\text { White R. at } \\
\text { Mile 1.2, Ark. }\end{array}$ & Dipped & 38.5 & 0 & 35.2 & 1 & -- & - & - \\
\hline $5-05$ & $\begin{array}{l}\text { Arkansas R. at } \\
\text { Mile 0.0, Ark. }\end{array}$ & Dipped & 41.0 & 0 & $\begin{array}{l}\text { Unmea- } \\
\text { sured }\end{array}$ & 1 & -- & -- & - \\
\hline 5-07 & $\begin{array}{l}\text { Yazoo R. at } \\
\text { Mile } \sim 3.0, \text { Miss. }\end{array}$ & Dipped & 42.2 & 0 & $\begin{array}{l}\text { Unmea- } \\
\text { sured }\end{array}$ & 1 & -- & - & - \\
\hline \multirow[t]{3}{*}{$5-06$} & Mississippi R. below & Integration & 58.9 & 41.8 & 41.8 & 18 & $1 / 8$ & 9.0 & 60 \\
\hline & Vicksburg, Miss. & Pumped & 12.0 & 0 & 0 & 9 & -- & -- & -- \\
\hline & & Pumped & 509 & 509 & 509 & 18 & -- & -. & 98 \\
\hline \multirow[t]{3}{*}{$5-08$} & Mississippi R. near & Integration & 60.9 & 45.2 & 45.2 & 16 & $3 / 16$ & 13.0 & 60 \\
\hline & St. Francisville, La. & Pumped & 12.0 & 0 & 0 & 8 & -- & -- & -- \\
\hline & & Pumped & 504 & 504 & 501 & 16 & -- & - & 87 \\
\hline \multirow[t]{3}{*}{$5-10$} & Mississippi R. below & Integration & 65.5 & 48.0 & 48.0 & 7 & $3 / 16$ & 13.0 & 52 \\
\hline & Belle Chasse, La. & Pumped & 12.0 & 0 & 0 & 7 & - & -- & -- \\
\hline & & Pumped & 508 & 508 & 508 & 7 & -- & - & 88 \\
\hline
\end{tabular}

\footnotetext{
${ }^{1}$ See table 15 for total suspended-sediment concentration of silt and clay $(<63 \mu \mathrm{m})$ which was dried at $105-110^{\circ} \mathrm{C}$. Data for the integration and small volume pumped samples were provided by John Garbarino. They do not include the sediment trapped in the centrifuge bowl bottom sealing unit; the silt and clay and colloid material were freeze dried. Data for the large volume pumped samples were provided by Colleen Rostad and do include the trapped sediment. The silt and clay were air dried at room temperature (about $23^{\circ} \mathrm{C}$ ) and the colloid material was freeze dried.

${ }^{2}$ Ship anchored at all verticals.

${ }^{3}$ Equal-discharge increments.
} 
The water and suspended-sediment mixture in the funnel was processed by allowir $\sigma$ it to drain at $2 \mathrm{~L} / \mathrm{min}$ into a continuous-flow centrifuge (Sharples, model AS-12; see Leenheer and others, 1989, for more details) spinning at about 16,000 r.p.m. Some sediment was retained on the Teflon centrifuge liner, some sediment was trapped in the centrifuge bowl bottom sealing unit, and some was elutriated from the centrifuge (see Moody and Meade, 1994, for more details). The elutriated water and remaining sediment was processed further by passing it through an ultrafiltration unit with pore sizas of $0.005 \mu \mathrm{m}$ (Rees and others, 1991). The sediment retained on the Teflon centrifuge liner and the sed iment trapped in the sealing unit were combined and called the pumped, composite silt and clay samples. This sample usually was extracted from a water volume of 500 to $1000 \mathrm{~L}$ and was used for organic contaminant analyses. At some sampling sites, a smaller volume of elutriated water and sediment was pronessed through the ultrafiltration unit than was processed through the centrifuge in order to reduce the processing time. The volume was based on the nature of the colloidal material and previous experience. The sediment retained on the membranes of the ultrafilter was called the pumped, composite colloid sample. The total mass of silt and clay and colloid material that was recovered is listed in table 10 for the Jul"-August 1991 cruise, in table 11 for the October-November 1991 cruise, and in table 12 for the April-May 1992 cruise as a percent of the suspended silt and clay concentration $(<63 \mu \mathrm{m})$ listed in tables $13-15$. 
[A and B are depth-integrated composites; $P$, pump composite collected when the water velocities were too slow for collecting a depth-integrated composite; Total sediment concentration of composites $A$ and $B$ were averaged in order to calculate a single value of sediment discharge; $\mathrm{m}^{3} / \mathrm{s}$, cubic meters per second; $\mathrm{mg} / \mathrm{L}$, milligrams per liter; $\mu \mathrm{m}$, micrometers; Analyses by R.H. Meade. Water discharge calculated by J.A. Moody]

\begin{tabular}{|c|c|c|c|c|c|c|c|c|c|c|}
\hline \multirow{2}{*}{$\begin{array}{l}\text { Date } \\
1991\end{array}$} & \multirow[b]{2}{*}{ Site name } & \multirow{2}{*}{$\begin{array}{l}\text { Com- } \\
\text { posite }\end{array}$} & \multirow{2}{*}{$\begin{array}{c}\text { Number } \\
\text { of } \\
\text { verticals }\end{array}$} & \multirow{2}{*}{$\begin{array}{c}\text { Water } \\
\text { dlscharge } \\
\left(\mathrm{m}^{3} / \mathrm{s}\right)\end{array}$} & \multicolumn{3}{|c|}{$\begin{array}{l}\text { Sediment concentration } \\
\text { (mg/L) }\end{array}$} & \multicolumn{3}{|c|}{$\begin{array}{l}\text { Sediment dlscharge } \\
\text { (metric tons/day) }\end{array}$} \\
\hline & & & & & $\begin{array}{l}\text { Silt and } \\
\text { clay } \\
<63 \mu \mathrm{m}\end{array}$ & $\begin{array}{l}\text { Sand } \\
>63 \mu \mathrm{m}\end{array}$ & Total & $\begin{array}{l}\text { Slit and } \\
\text { clay } \\
<63 \mu \mathrm{m}\end{array}$ & $\begin{array}{l}\text { Sand } \\
>63 \mu \mathrm{m}\end{array}$ & Total \\
\hline $7-05$ & $\begin{array}{l}\text { Mississippi R. above } \\
\text { St. Anthony Falls, Minn. }\end{array}$ & $\begin{array}{l}\text { A } \\
\text { B }\end{array}$ & $\begin{array}{l}25 \\
25\end{array}$ & 470 & $\begin{array}{l}30 \\
31\end{array}$ & $\begin{array}{l}2 \\
2\end{array}$ & $\begin{array}{l}32 \\
33\end{array}$ & 1,200 & 80 & 1,300 \\
\hline $7-06$ & $\begin{array}{l}\text { Minnesota } R \text {. at } \\
\text { Mile 3.5, Minn. }\end{array}$ & $\begin{array}{l}\text { A } \\
\text { B }\end{array}$ & $\begin{array}{l}25 \\
25\end{array}$ & 600 & $\begin{array}{l}103 \\
103\end{array}$ & $\begin{array}{l}71 \\
71\end{array}$ & $\begin{array}{l}174 \\
174\end{array}$ & 5,340 & 3,680 & 9,020 \\
\hline $7-08$ & $\begin{array}{l}\text { Mississippi R. at } \\
\text { Hastings, Minn. }\end{array}$ & $\begin{array}{l}\text { A } \\
\text { B }\end{array}$ & $\begin{array}{l}{ }^{3} 8 \\
{ }^{3} 8\end{array}$ & 980 & $\begin{array}{l}82 \\
83\end{array}$ & $\begin{array}{l}0.3 \\
0.3\end{array}$ & $\begin{array}{l}82 \\
83\end{array}$ & 6,940 & 30 & 6,970 \\
\hline $7-10$ & $\begin{array}{l}\text { Mississippi R. near } \\
\text { Pepin, Wis. }\end{array}$ & $\begin{array}{l}\text { A } \\
\text { B }\end{array}$ & $\begin{array}{l}12 \\
12\end{array}$ & 1,350 & $\begin{array}{l}9 \\
9\end{array}$ & $\begin{array}{l}{ }^{4} 0 \\
{ }^{4} 0\end{array}$ & $\begin{array}{l}9 \\
9\end{array}$ & 1,000 & 0 & 1,000 \\
\hline $7-12$ & $\begin{array}{l}\text { Mississippi R, at } \\
\text { Trempealeau, Wis. }\end{array}$ & $\begin{array}{l}\text { A } \\
\text { B }\end{array}$ & $\begin{array}{l}{ }_{3}^{3} 8 \\
{ }^{3} 8\end{array}$ & 1,440 & $\begin{array}{l}28 \\
28\end{array}$ & $\begin{array}{l}{ }^{4} 0 \\
{ }^{4} 0\end{array}$ & $\begin{array}{l}28 \\
28\end{array}$ & 3,500 & 0 & 3,500 \\
\hline $7-15$ & $\begin{array}{l}\text { Mississippi R. below } \\
\text { Lock and Dam 9, Wis. }\end{array}$ & $\begin{array}{l}\text { A } \\
\text { B }\end{array}$ & $\begin{array}{l}39 \\
39\end{array}$ & 1,590 & $\begin{array}{l}72 \\
74\end{array}$ & $\begin{array}{l}0.2 \\
0.2\end{array}$ & $\begin{array}{l}72 \\
74\end{array}$ & 10,000 & 30 & 10,000 \\
\hline $7-18$ & $\begin{array}{l}\text { Mississippi R. at } \\
\text { Clinton, lowa }\end{array}$ & $\begin{array}{l}\text { A } \\
\text { B }\end{array}$ & $\begin{array}{l}39 \\
39\end{array}$ & 1,850 & $\begin{array}{l}68 \\
65\end{array}$ & ${ }^{5}<0.1$ & $\begin{array}{l}68 \\
65\end{array}$ & 10,500 & $<16$ & 10,500 \\
\hline $7-21$ & $\begin{array}{l}\text { Mississippi R. at } \\
\text { Keokuk, lowa }\end{array}$ & $\begin{array}{l}\text { A } \\
\text { B }\end{array}$ & $\begin{array}{l}39 \\
39\end{array}$ & 2,050 & $\begin{array}{l}46 \\
44\end{array}$ & $\begin{array}{l}4_{0} 0 \\
4_{0}\end{array}$ & $\begin{array}{l}46 \\
44\end{array}$ & 8,000 & 0 & 8,000 \\
\hline $7-24$ & $\begin{array}{l}\text { Mississippi R. near } \\
\text { Winfield, Mo. }\end{array}$ & $\begin{array}{l}\text { A } \\
\text { B }\end{array}$ & $\begin{array}{l}{ }^{3} 10 \\
{ }^{3} 10\end{array}$ & 2,730 & $\begin{array}{l}74 \\
74\end{array}$ & $\begin{array}{l}0.2 \\
0.2\end{array}$ & $\begin{array}{l}74 \\
74\end{array}$ & 17,500 & 50 & 17,500 \\
\hline $7-25$ & $\begin{array}{l}\text { Illinois R. at } \\
\text { Hardin, lll. }\end{array}$ & $\mathbf{P}$ & 1 & 260 & 47 & ${ }^{4} 0$ & 47 & 1,100 & 0 & 1,100 \\
\hline $7-27$ & $\begin{array}{l}\text { Missouri R. at } \\
\text { St. Charles, Mo. }\end{array}$ & $\begin{array}{l}\text { A } \\
\text { B }\end{array}$ & $\begin{array}{l}15 \\
15\end{array}$ & 1,100 & $\begin{array}{l}109 \\
108\end{array}$ & $\begin{array}{l}31 \\
31\end{array}$ & $\begin{array}{l}140 \\
139\end{array}$ & 10,300 & 2,900 & 13,200 \\
\hline $7-29$ & $\begin{array}{l}\text { Mississippi R. at } \\
\text { Thebes, Ill. }\end{array}$ & $\begin{array}{l}\text { A } \\
\text { B }\end{array}$ & $\begin{array}{l}15 \\
15\end{array}$ & 4,390 & $\begin{array}{l}82 \\
83\end{array}$ & $\begin{array}{l}24 \\
22\end{array}$ & $\begin{array}{l}106 \\
105\end{array}$ & 31,100 & 8,700 & 39,800 \\
\hline $7-30$ & $\begin{array}{l}\text { Ohio R. at } \\
\text { Olmsted, } 111 .\end{array}$ & $\mathbf{P}$ & 15 & 2.410 & 22 & ${ }^{4} 0$ & 22 & 4,600 & 0 & 4,600 \\
\hline $8-03$ & $\begin{array}{l}\text { Mississippi R. below } \\
\text { Vicksburg, Miss. }\end{array}$ & $\begin{array}{l}\text { A } \\
\text { B }\end{array}$ & $\begin{array}{l}17 \\
17\end{array}$ & 8,750 & $\begin{array}{l}105 \\
111\end{array}$ & $\begin{array}{l}2.7 \\
3.3\end{array}$ & $\begin{array}{l}108 \\
114\end{array}$ & 81,600 & 2,300 & 83,900 \\
\hline $8-05$ & $\begin{array}{l}\text { Mississippi R. near } \\
\text { St. Francisville, La. }\end{array}$ & $\begin{array}{l}\mathrm{A} \\
\mathrm{B}\end{array}$ & $\begin{array}{l}15 \\
15\end{array}$ & 6,190 & $\begin{array}{l}111 \\
113\end{array}$ & $\begin{array}{l}4.2 \\
4.4\end{array}$ & $\begin{array}{l}115 \\
117\end{array}$ & 59,900 & 2,300 & 62,200 \\
\hline $8-07$ & $\begin{array}{l}\text { Mississippi R. below } \\
\text { Belle Chasse, La. }\end{array}$ & $\mathbf{P}$ & 5 & 4,340 & 42 & 0 & 42 & 16,000 & 0 & 14,000 \\
\hline
\end{tabular}


Table 14.--Concentrations of suspended sediment in composite samples collectid from the Mississippi River and some of its tributaries and the corresponding sediment $c^{\text {"scharges }}$ for October-November 1991 cruise

[A and B are depth-integrated composites; P, pump composite collected when the water velocities were too slow for collecting a depth-integrated composite; Total sed 'ment concentration of composites $\mathrm{A}$ and $\mathrm{B}$ were averaged in order to calculate a single value of sediment discharge; $\mathrm{m}^{3} / \mathrm{s}$, cubic meters per second; $\mathrm{mg} / \mathrm{L}$, milligrams per l'ter; $\mu \mathrm{m}$, micrometers; Analyses by R.H. Meade. Water discharge measured by J.A. 1 1oody]

\begin{tabular}{|c|c|c|c|c|c|c|c|c|c|c|}
\hline \multirow{2}{*}{$\begin{array}{l}\text { Date } \\
1991\end{array}$} & \multirow[b]{2}{*}{ Slte name } & \multirow{2}{*}{$\begin{array}{l}\text { Com- } \\
\text { posite }\end{array}$} & \multirow{2}{*}{$\begin{array}{c}\text { Number } \\
\text { of } \\
\text { verticals }\end{array}$} & \multirow{2}{*}{$\begin{array}{c}\text { Water } \\
\text { discharge } \\
\left(\mathrm{m}^{3} / \mathrm{s}\right)\end{array}$} & \multicolumn{3}{|c|}{$\begin{array}{l}\text { Sediment concentration } \\
\text { ( } \mathbf{m g} / \mathrm{L})\end{array}$} & \multicolumn{3}{|c|}{$\begin{array}{l}\text { Sediment discharge } \\
\text { (metrir tons/day) }\end{array}$} \\
\hline & & & & & $\begin{array}{l}\text { Silt and } \\
\text { clay } \\
<63 \mu \mathrm{m}\end{array}$ & $\begin{array}{l}\text { Sand } \\
>63 \mu \mathrm{m}\end{array}$ & Total & $\begin{array}{l}\text { Silt and } \\
\text { clay } \\
<63 \mu \mathrm{m}\end{array}$ & $\begin{array}{l}\text { Sand } \\
>\$ 3 \mu \mathrm{m}\end{array}$ & Total \\
\hline $10-07$ & $\begin{array}{l}\text { Mississippi R. above } \\
\text { St. Anthony Falls, Minn. }\end{array}$ & $\bar{A}$ & 5 & 220 & 12 & 0.1 & 12 & 230 & 2 & 230 \\
\hline $10-08$ & $\begin{array}{l}\text { Minnesota R. at } \\
\text { Mile 3.5, Minn. }\end{array}$ & $\begin{array}{l}\mathrm{A} \\
\mathrm{P}\end{array}$ & $\begin{array}{l}5 \\
5\end{array}$ & 130 & $\begin{array}{l}61 \\
62\end{array}$ & $\begin{array}{c}0.1 \\
\text { no } \\
\text { sample }\end{array}$ & $\begin{array}{l}61 \\
62\end{array}$ & 690 & 1 & 690 \\
\hline $10-10$ & $\begin{array}{l}\text { Mississippi R. at } \\
\text { Hastings, Minn. }\end{array}$ & $\mathbf{P}$ & 5 & 350 & 45 & $<0.1$ & 45 & 1,400 & $<3$ & 1,400 \\
\hline $10-13$ & $\begin{array}{l}\text { Mississippi R. near } \\
\text { Pepin, Wis. }\end{array}$ & $\mathbf{P}$ & 6 & 510 & 9 & $\sim 0$ & 9 & 400 & $\sim 0$ & 400 \\
\hline $10-15$ & $\begin{array}{l}\text { Mississippi R. at } \\
\text { Trempealeau, Wis. }\end{array}$ & $\mathbf{P}$ & 7 & 660 & 12 & $<0.1$ & 12 & 680 & $<6$ & 680 \\
\hline $10-18$ & $\begin{array}{l}\text { Mississippi R. below } \\
\text { Lock and Dam 9, Wis. }\end{array}$ & $\mathbf{P}$ & 6 & 690 & 28 & $<0.1$ & 28 & 1,700 & $<6$ & 1,700 \\
\hline $10-22$ & $\begin{array}{l}\text { Mississippi R. at } \\
\text { Clinton, Jowa }\end{array}$ & $\mathbf{P}$ & 6 & 940 & 31 & $<0.1$ & 31 & 2,500 & $<8$ & 2,500 \\
\hline $10-27$ & $\begin{array}{l}\text { Mississippi R. at } \\
\text { Keokuk, lowa }\end{array}$ & $\mathbf{P}$ & 7 & 1,410 & 32 & $<0.1$ & 32 & 3,900 & $<10$ & 3,900 \\
\hline $10-30$ & $\begin{array}{l}\text { Mississippi R. near } \\
\text { Winfield, Mo. }\end{array}$ & $\mathbf{P}$ & 7 & 1,230 & 36 & $<0.1$ & 36 & 3,800 & $<10$ & 3,800 \\
\hline $10-31$ & $\begin{array}{l}\text { Illinois R. at } \\
\text { Hardin, Ill. }\end{array}$ & $P$ & 1 & 520 & 143 & 0.1 & 143 & 6,420 & 4 & 6,420 \\
\hline $11-03$ & $\begin{array}{l}\text { Missouri R. at } \\
\text { St. Charles, Mo. }\end{array}$ & $\begin{array}{l}\text { A } \\
\text { B }\end{array}$ & $\begin{array}{l}10 \\
10\end{array}$ & 1,350 & $\begin{array}{l}164 \\
162\end{array}$ & $\begin{array}{l}145 \\
142\end{array}$ & $\begin{array}{l}309 \\
304\end{array}$ & 19,000 & 16,800 & 35,800 \\
\hline $11-05$ & $\begin{array}{l}\text { Mississippi R. at } \\
\text { Thebes, Ill. }\end{array}$ & $\begin{array}{l}\text { A } \\
\text { B }\end{array}$ & $\begin{array}{l}12 \\
12\end{array}$ & 3,870 & $\begin{array}{l}81 \\
79\end{array}$ & $\begin{array}{l}68 \\
52\end{array}$ & $\begin{array}{l}149 \\
131\end{array}$ & 26,700 & 20,100 & 46,800 \\
\hline $11-06$ & $\begin{array}{l}\text { Ohio R. at } \\
\text { Olmsted, Ill. }\end{array}$ & $\mathbf{P}$ & 11 & 2,480 & 19 & 0.6 & 20 & 4,100 & 130 & 4,200 \\
\hline $11-09$ & $\begin{array}{l}\text { Mississippi R. below } \\
\text { Vicksburg, Miss. }\end{array}$ & $\begin{array}{l}\text { A } \\
\text { B }\end{array}$ & $\begin{array}{l}13 \\
13\end{array}$ & 10,700 & $\begin{array}{l}155 \\
152\end{array}$ & $\begin{array}{l}27 \\
30\end{array}$ & $\begin{array}{l}182 \\
182\end{array}$ & 142,000 & 26,000 & 168,000 \\
\hline $11-11$ & $\begin{array}{l}\text { Mississippi R. near } \\
\text { St. Francisville, La. }\end{array}$ & $\begin{array}{l}\text { A } \\
\text { B }\end{array}$ & $\begin{array}{l}12 \\
12\end{array}$ & 8,950 & $\begin{array}{l}173 \\
172\end{array}$ & $\begin{array}{l}36 \\
37\end{array}$ & $\begin{array}{l}209 \\
209\end{array}$ & 133,000 & 28,000 & 161,000 \\
\hline $11-13$ & $\begin{array}{l}\text { Mississippi R. below } \\
\text { Belle Chasse, La. }\end{array}$ & $\begin{array}{l}A \\
P\end{array}$ & $\begin{array}{l}5 \\
5\end{array}$ & $\begin{array}{l}8,840 \\
8,840\end{array}$ & $\begin{array}{l}142 \\
112\end{array}$ & $\begin{array}{l}0.1 \\
0.1\end{array}$ & $\begin{array}{l}142 \\
112\end{array}$ & $\begin{array}{r}108,000 \\
85,500\end{array}$ & $\begin{array}{l}80 \\
80\end{array}$ & $\begin{array}{r}108,000 \\
85,600\end{array}$ \\
\hline
\end{tabular}

\footnotetext{
'The $A$ and $B$ samples were collected at the same locations.
} 
Table 15.-Concentrations of suspended sediment in composite sampies coliected from the Mississippi River and some of its tributaries and the corresponding sediment discharges for April-May 1992 cruise

[A and B are depth-integrated composites; P, pump composite collected when the water velocities were too slow for collecting a depth-integrated composite; Total sediment concentration of composites A and B were averaged in order to calculate a single value of sediment discharge; $\mathrm{m}^{3} / \mathrm{s}$, cubic meters per second; $\mathrm{mg} / \mathrm{L}$, milligrams per liter; $\mu \mathrm{m}$, micrometer; Analyses by R.H. Meade. Water discharge calculated by J.A. Moody]

\begin{tabular}{|c|c|c|c|c|c|c|c|c|c|c|}
\hline \multirow{2}{*}{$\begin{array}{l}\text { Date } \\
1992\end{array}$} & \multirow[b]{2}{*}{ Site name } & \multirow{2}{*}{$\begin{array}{l}\text { Com- } \\
\text { posite }\end{array}$} & \multirow{2}{*}{$\begin{array}{c}\text { Number } \\
\text { of } \\
\text { verticals } 1\end{array}$} & \multirow{2}{*}{$\begin{array}{c}\text { Water } \\
\text { discharge } \\
\left(\mathrm{m}^{3} / \mathrm{s}\right)\end{array}$} & \multicolumn{3}{|c|}{$\begin{array}{l}\text { Sediment concentration } \\
(\mathbf{m g} / \mathbf{L})\end{array}$} & \multicolumn{3}{|c|}{$\begin{array}{l}\text { Sediment discharce } \\
\text { (metric tons/day) }\end{array}$} \\
\hline & & & & & $\begin{array}{c}\text { Silt and } \\
\text { clay } \\
<63 \mu \mathrm{m}\end{array}$ & $\begin{array}{c}\text { Sand } \\
>63 \mu \mathrm{m}\end{array}$ & Total & $\begin{array}{l}\text { Silt and } \\
\text { clay } \\
<63 \mu \mathrm{m}\end{array}$ & $\begin{array}{l}\text { Sand } \\
>63 \mu \mathrm{m}\end{array}$ & Total \\
\hline $4-06$ & $\begin{array}{l}\text { Mississippi R. above } \\
\text { St. Anthony Falls, Minn. }\end{array}$ & $\begin{array}{l}\text { A } \\
\text { B }\end{array}$ & $\begin{array}{l}25 \\
25\end{array}$ & 310 & $\begin{array}{l}12 \\
12\end{array}$ & $\begin{array}{l}1.3 \\
1.2\end{array}$ & $\begin{array}{l}13 \\
13\end{array}$ & 320 & 32 & 350 \\
\hline 4-08 & $\begin{array}{l}\text { Minnesota R. at } \\
\text { Mile 3.5, Minn. }\end{array}$ & $\begin{array}{l}\text { A } \\
\text { B }\end{array}$ & $\begin{array}{l}34 \\
34\end{array}$ & 260 & $\begin{array}{l}95 \\
97\end{array}$ & $\begin{array}{l}10.5 \\
10.3\end{array}$ & $\begin{array}{l}105 \\
107\end{array}$ & 2,160 & 230 & 2,390 \\
\hline $4-10$ & $\begin{array}{l}\text { Mississippi R. at } \\
\text { Hastings, Minn. }\end{array}$ & A & 7 & 570 & 36 & $<0.1$ & 36 & 1,800 & $<5$ & 1,800 \\
\hline $4-12$ & $\begin{array}{l}\text { Mississippi R. near } \\
\text { Pepin, Wis. }\end{array}$ & $\mathbf{P}$ & 7 & 950 & 12 & ${ }^{4} 0$ & 12 & 980 & $\mathbf{0}$ & 980 \\
\hline $4-14$ & $\begin{array}{l}\text { Mississippi R. at } \\
\text { Trempealeau, Wis. }\end{array}$ & $\begin{array}{l}\text { A } \\
\text { B }\end{array}$ & $\begin{array}{l}37 \\
37\end{array}$ & 1,330 & $\begin{array}{l}15 \\
14\end{array}$ & 0.1 & $\begin{array}{l}15 \\
14\end{array}$ & 1,600 & 10 & 1,600 \\
\hline $4-17$ & $\begin{array}{l}\text { Mississippi R. below } \\
\text { Lock and Dam 9, Wis. }\end{array}$ & $\begin{array}{l}\text { A } \\
\text { B }\end{array}$ & $\begin{array}{l}{ }^{3} 7 \\
3_{7}\end{array}$ & 1,590 & $\begin{array}{l}24 \\
24\end{array}$ & $\begin{array}{l}0.2 \\
0.3\end{array}$ & $\begin{array}{l}24 \\
24\end{array}$ & 3,300 & 30 & 3,300 \\
\hline $4-19$ & $\begin{array}{l}\text { Mississippi R. at } \\
\text { Clinton, lowa }\end{array}$ & $\begin{array}{l}\text { A } \\
\text { B }\end{array}$ & $\begin{array}{l}38 \\
38\end{array}$ & 2,320 & $\begin{array}{l}40 \\
40\end{array}$ & $\begin{array}{l}0.1 \\
0.1\end{array}$ & $\begin{array}{l}40 \\
40\end{array}$ & 8,000 & 20 & 8,000 \\
\hline $4-23$ & $\begin{array}{r}\text { Mississippi R. at } \\
\text { Keokuk, lowa }\end{array}$ & $\begin{array}{l}\text { A } \\
\text { B }\end{array}$ & $\begin{array}{l}7 \\
7\end{array}$ & 4,220 & $\begin{array}{l}298 \\
300\end{array}$ & $\begin{array}{l}0.2 \\
0.3\end{array}$ & $\begin{array}{l}298 \\
300\end{array}$ & 109,000 & 90 & $1 C 9,000$ \\
\hline $4-26$ & $\begin{array}{l}\text { Mississippi R. near } \\
\text { Winfield, Mo. }\end{array}$ & $\begin{array}{l}\text { A } \\
\text { B }\end{array}$ & $\begin{array}{l}10 \\
10\end{array}$ & 5,070 & $\begin{array}{l}295 \\
291\end{array}$ & $\begin{array}{l}15 \\
16\end{array}$ & $\begin{array}{l}310 \\
307\end{array}$ & 128,000 & 7,000 & 135,000 \\
\hline $4-27$ & $\begin{array}{l}\text { Illinois R. at } \\
\text { Hardin, Ill. }\end{array}$ & $\begin{array}{l}\text { A } \\
\text { B }\end{array}$ & $\begin{array}{l}35 \\
3_{5}\end{array}$ & 860 & $\begin{array}{l}235 \\
225\end{array}$ & $\begin{array}{l}0.3 \\
0.4\end{array}$ & $\begin{array}{l}235 \\
225\end{array}$ & 17,100 & 30 & 17,100 \\
\hline $4-29$ & $\begin{array}{l}\text { Missouri R, at } \\
\text { St. Charles, Mo. }\end{array}$ & $\begin{array}{l}\text { A } \\
\text { B }\end{array}$ & $\begin{array}{l}8 \\
8\end{array}$ & 3,560 & $\begin{array}{l}1,180 \\
1,180\end{array}$ & $\begin{array}{l}169 \\
192\end{array}$ & $\begin{array}{l}1,349 \\
1,372\end{array}$ & 363,000 & 55,400 & 418,000 \\
\hline $5-01$ & $\begin{array}{l}\text { Mississippi R. at } \\
\text { Thebes, } 111 .\end{array}$ & $\begin{array}{l}\text { A } \\
\text { B }\end{array}$ & $\begin{array}{l}9 \\
9\end{array}$ & 10,500 & $\begin{array}{l}591 \\
610\end{array}$ & $\begin{array}{r}113 \\
88\end{array}$ & $\begin{array}{l}704 \\
698\end{array}$ & 544,000 & 90,700 & 635,000 \\
\hline $5-03$ & Ohio R. at Olmsted, III. & $P$ & 11 & 6,150 & 67 & 0.3 & 67 & 35,600 & 200 & $3 ؟, 800$ \\
\hline $5-06$ & $\begin{array}{l}\text { Mississippi R. below } \\
\text { Vicksburg, Miss. }\end{array}$ & $\begin{array}{l}\text { A } \\
\text { B }\end{array}$ & $\begin{array}{l}59 \\
59\end{array}$ & 21,700 & $\begin{array}{l}296 \\
304\end{array}$ & $\begin{array}{l}55 \\
61\end{array}$ & $\begin{array}{l}351 \\
365\end{array}$ & 562,000 & 109,000 & 671,000 \\
\hline $5-08$ & $\begin{array}{l}\text { Mississippi R. near } \\
\text { St. Francisville, La. }\end{array}$ & $\begin{array}{l}\text { A } \\
\text { B }\end{array}$ & $\begin{array}{l}5_{8} \\
5_{8}\end{array}$ & 15,100 & $\begin{array}{l}297 \\
297\end{array}$ & $\begin{array}{l}38 \\
38\end{array}$ & $\begin{array}{l}335 \\
335\end{array}$ & 387,000 & 50,000 & 437,000 \\
\hline $5-10$ & $\begin{array}{c}\text { Mississippi R. below } \\
\text { Belle Chasse, La. }\end{array}$ & $\begin{array}{l}\mathrm{A} \\
\mathrm{B} \\
\end{array}$ & $\begin{array}{l}7 \\
7 \\
\end{array}$ & 14,500 & $\begin{array}{l}300 \\
305 \\
\end{array}$ & $\begin{array}{l}9 \\
8 \\
\end{array}$ & $\begin{array}{l}309 \\
313 \\
\end{array}$ & 378,000 & 10,000 & 388,000 \\
\hline
\end{tabular}

\footnotetext{
${ }^{1}$ The $A$ and $B$ samples were collected at the same locations.

${ }^{2} \mathrm{~A}$ and $\mathrm{B}$ sample bottles were lowered different numbers of times at different verticals to obtain equal-discharge-increment sample.

${ }^{3} \mathrm{~A}$ and $\mathrm{B}$ sample bottles were both lowered twice at each vertical.

${ }^{4}$ Visual estimate.

${ }^{5}$ The $A$ and $B$ samples were collected at different locations.
} 


\section{Depth-Integrated Composite Sample}

The equal-width-increment (equal-transit-rate), depth-integration method (Nord 'n and others, 1983; Richey and others, 1986; Meade and Stevens, 1990) was used to collect a depth-integrated composite sample of water and suspended sediment from 1 to 18 vertical locations arross the river. A collapsible-bag sampler (Stevens and others, 1980) fitted with an isokinetic nozzle (Gurr and Norman, 1970, p. 8-10) collected suspended sediment in sand, silt, and clay sizes (see tables 10, 11, and 12 for nozzle sizes). The sampler was held in a frame so that the nozzle was horizontal, faced into the current, and was about $45 \mathrm{~cm}$ above the bottom of a sounding weight (fig. 5). A Price AA current meter was mounted $22 \mathrm{~cm}$ above the nozzle of the sampler. The current meter was used to measure the depth-averaged velocity and to determine the nozzle efficiency (volume of water collected divide $t$ by the mean velocity, the cross-sectional area of the nozzle, and the integration time). The vertical transit rate was controlled by a hydraulic winch, which produced a constant transit rate for the downcast and for the upcast. The multiple gears on the hydraulic system allowed transit rates as slow as $2 \mathrm{~cm} / \mathrm{s}$ and as fast as $17 \mathrm{~cm} / \mathrm{s}$. The same transit rate was reproduced within $1 \mathrm{~cm} / \mathrm{s}$ for each vertical acros: the river and was verified with a hand-held tachometer at the beginning of each downcast and at th? beginning of each upcast.

Two 8-L plastic bottles, identified as A and B, with collapsible, perflourinated-alkoxy (PFA) Teflon bags and fluorinated-ethylene-propylene (FEP) Teflon nozzles were used in the isokinetic sampler to collect individual samples at alternate verticals, which were combined into two separate, depth-integrated, composite samples. During previous cruises summarized earlier (Moody anc Meade, 1992, 1993), bottle $A$ was used to collect samples at the odd-numbered verticals and bottle $B$ at the evennumbered verticals. However, during the cruises of 1991-92 reported here, both bottles usually were used to collect samples at the same vertical, and at some sites they were used repeatedly at the same vertical in order to obtain the necessary volume. The A and B samples were poured into separate glass, graduated cylinders through separate, nickel-mesh, $63-\mu \mathrm{m}$ sieves to remove the send. This sand was saved and called the depth-integrated, composite sand sample. The water volume collected at each individual vertical was measured and then composited in one of two separate Teflon-coted, stainlesssteel, churn splitters labeled A and B (see tables 10, 11, and 12 for total volume of water collected). The only significant difference between these procedures and those described for the low-water cruises by Leenheer and others (1989) and Moody and Meade (1992) is the substitution of the stronger, but less flexible, PFA Teflon bags for the previously used FEP Teflon bags. Leenheer and others (198?) describe the Teflon bags, nickel sieves, and churn splitters in more detail and discuss the evaluaticn of materials considered for the fabrication of each item so they would meet the diverse requirements for suspendedsediment, trace-organic, and trace-element analysis.

The remaining water and suspended sediment was processed further by keeping the suspended sediment in suspension by churning, and different-sized subsamples were taken from the $A$ and $B$ churn splitters for determining suspended-sediment concentration (150-250 mL) and particle-size distribution (4-24 L) and for analysis of major ions, trace elements, organic compounds, and nutrients. The remaining water and suspended sediment in the A and B churn splitters was combined and processed through the continuous-flow centrifuge described earlier. The sediment retained on the Teflon centrifuge liner was saved for trace-element analyses and called the depth-integrated, composite silt and clay sample. The sediment trapped on the centrifuge bowl bottom sealing unit may have been contaminated by coming in contact with metal parts which were not coated with Teflon. Therefore, this trapped sediment was not combined with the sediment from the Teflon centrifuge liner as was done for the pumped, composite silt and clay samples but was saved as a separate sample called the reject sample. The water and sediment elutriated from the centrifuge was processed further by ultrafiltration to cbtain a depth- 
integrated colloid sample. The total mass of silt and clay (which does not include the trapped sediment) and colloid material recovered is listed in tables $10-12$ as a percent of the suspended silt and clay concentration $(<63 \mu \mathrm{m})$ listed in tables 13-15. A generalized flow diagram for processing the depth-inte;rated composite sample is shown in figure 6.

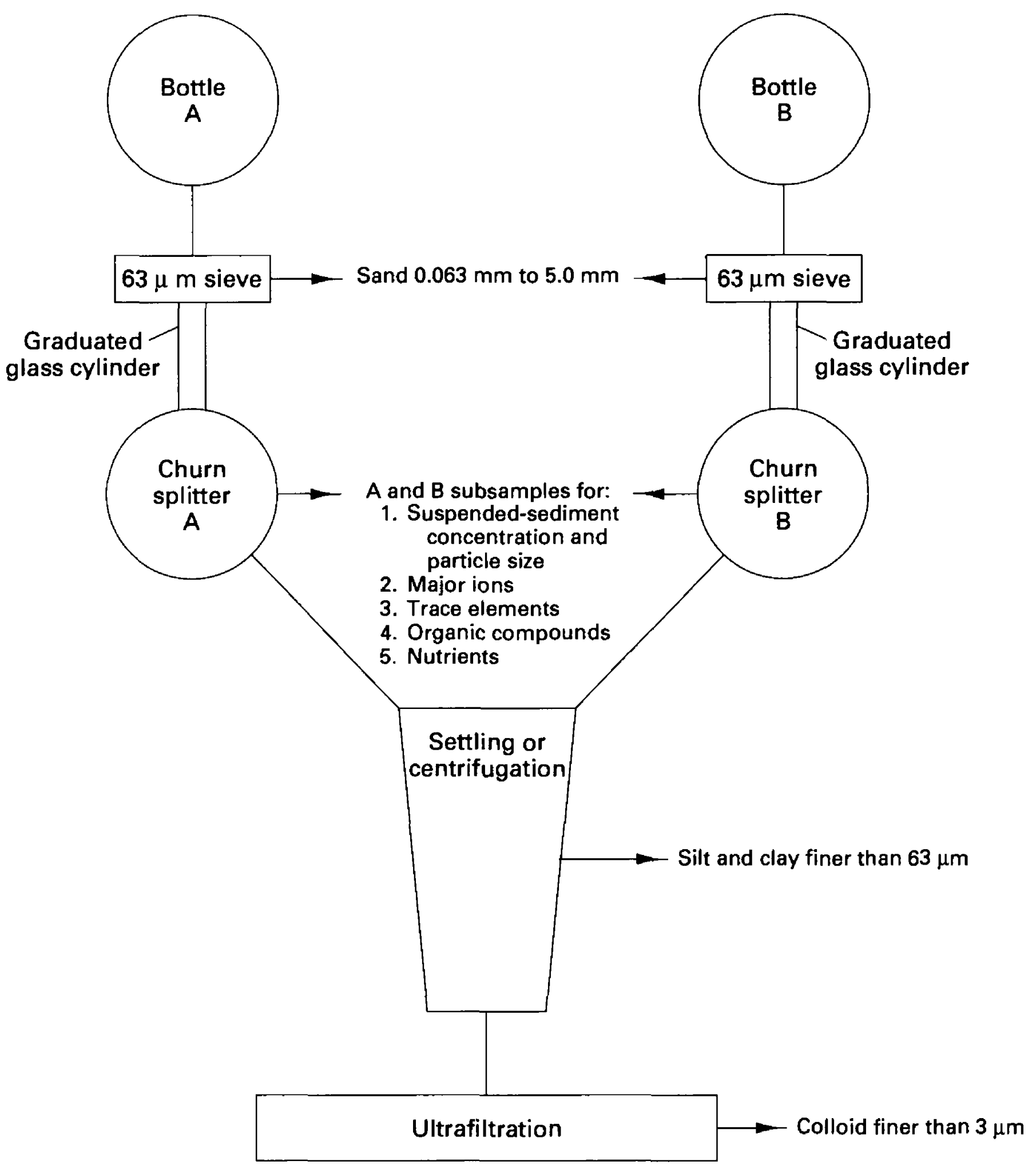

Figure 6.--Flow diagram for processing the suspended-sediment sample collected by the depth-integration method. 


\section{Suspended-Sediment Analysis}

The suspended sediment collected by using the depth-integration method was split into a sand fraction consisting of particle diameters greater than $63 \mu \mathrm{m}$ and a fine fraction consisting of si " and clay-size particles with diameters finer than $63 \mu \mathrm{m}$. The particle concentrations of the sand and the silt and clay fractions were measured separately and then added together to determine the total particle concentration.

The particle-size analysis of the sand and the silt and clay fraction used different meth $x$ ds. However, the results were combined to give particle-size distribution for the entire composite suspended-sediment sample collected at each sampling site.

\section{Concentratlon}

There were two replicate suspended-sand samples (composites A and B) for most sampling sites. If there was not enough sand in either the A or B composite samples, the two samples were combined to form a single suspended-sand sample. The sands were transferred to glass jars and transported to a USGS laboratory in Denver, Colorado, where they were dried at $80^{\circ} \mathrm{C}$, weighed to within $0.1 \mathrm{mg}$, and stored in polypropylene bottles for particle-size analysis. The masses ranged from $1 \mathrm{mg}$ to about 5,000 $\mathrm{mg}$. The collected volume of water was about $50 \mathrm{~L}$ (measured to $0.1 \mathrm{~L}$ ) so that the sand concentrations in tables 13-15 have analytical errors ranging from about 2 percent for concentrations of $0.1 \mathrm{mg} / \mathrm{L}$ to less than 0.1 percent for concentrations of $100 \mathrm{mg} / \mathrm{L}$. The mean percent difference between tho two replicate sand concentrations for all three cruises ranged from 0 to 20 percent with an average of 7 percent based on the sampling sites that had more than $1 \mathrm{mg} / \mathrm{L}$ of sand.

Two subsamples (about 150-250 mL) were taken from composite A and composite B for determining the suspended-sediment concentration of material finer than $63 \mu \mathrm{m}$. Each of the four subsamples was filtered through paired, preweighed Millipore HA filters $\left(0.45-\mu \mathrm{m}\right.$ pore size) and dried at about $40^{\circ} \mathrm{C}$ on board the research vessel. Later, in a laboratory ashore, the filter with sediment was drie $\mathrm{d}$ at $105-110^{\circ} \mathrm{C}$ and reweighed to within $0.05 \mathrm{mg}$, and the preweight of the filter was subtracted to obtain the mass of silt and clay, which typically was 20 to $40 \mathrm{mg}$. The volume of each subsample was measured to within $\pm 1 \mathrm{~mL}$, so that the concentrations in tables $13-15$ have an analytical error of \pm 1 percent. or typically 1 $\mathrm{mg} / \mathrm{L}$.

The mean range in silt and clay concentration (calculated from two to four replicate samples at each sampling site) was $3 \pm 3$ percent of the mean silt and clay concentration for the July-Augıst 1991 cruise, $2 \pm 3$ percent for the October-November 1991 cruise, and $3 \pm 2$ percent for the April-May 1092 cruise. The mean percent difference between the silt and clay concentrations of the two replicates (composites $A$ and $B$ ) for all sampling sites was about $2 \pm 4$ percent of the mean silt and clay concentration for all three cruises with a relative standard deviation of \pm 4 percent.

The suspended-sediment concentrations were multiplied by the water discharges (listed in the fifth column of tables 13-15) and by a unit-conversion constant ( 0.0864$)$ to obtain the sediment discharges in metric tons per day listed in the last three columns of tables 13-15. 


\section{Particle Size}

Particle-size distributions of the suspended sediment (from 1 to $1,000 \mu \mathrm{m}$ ) for the three cruises are listed in tables 16-18. The particle-size distributions of the suspended sand (greater than $63 \mu \mathrm{m}$ ) were analyzed by the visual-accumulation tube method, and the particle-size distribution of the suspended silt and clay (finer than $63 \mu \mathrm{m}$ ) were analyzed by the SediGraph method. Both methods are based on settling properties of the particles. Both methods involve preliminary chemical treatment that disaggregates the particles as they exist in the river. Therefore, the size analyses reported here are more representative of the assemblages of individual particles available to interact with the dissolved matter in the river and less representative of the hydraulic properties of the grains as they are transported by the river. Stallard and Martin (1989) noted that settling velocities of suspended sediments that were chemically dispersed for analysis, such as those reported here, were markedly slower than settling velocities of suspended sediments that were allowed to settle in native river water immediately after sampliro.

The sand samples that were saved in separate polypropylene bottles were sent to the USGS sediment laboratory in Iowa City, Iowa, for size analysis by the visual-accumulation-tube method (Guy, 1969). Replicate samples were run, and if the results did not agree within 5 percent for all size classes, the analysis was repeated (Matthes and others, 1992). A complication arose during the particle-size analysis when the sand samples were treated with hydrogen peroxide, the standard technique for rem oving organic matter (Guy, 1969, p. 52). This treatment apparently disaggregated silt particles from sard-size aggregates. In most of the suspended-sand samples, the hydrogen peroxide treatment released silt grains from sand-size aggregates. Although the quantities of released silt grains were almost always 2 percent or less of the total concentration of suspended sediment, they were subtracted, where appropriate, from the sand concentrations and added to the concentration of the coarsest silt fraction ( 32 to $63 \mu \mathrm{m})$ in the data reported in tables $16-18$.

The reproducibility of the sampling and size-analysis procedures was previously addressed by Moody and Meade (1992, table 18) for the sand fractions of the A and B composites of 12 suspendedsediment samples collected during 1987-88. When results were expressed as percentages of the sand fraction, differences between percents finer than certain sizes were as great as 7 percent. When results were expressed as percentages of the total sample, these differences were never more than 2 percent.

Only the samples collected by the depth-integration method were used in the particle-size analysis of suspended sediment finer than $63 \mu \mathrm{m}$ (except as indicated by footnote 3 in tables 16,17, and 18). The essential problem in preparing a silt and clay fraction sample for particle-size analysis is one of converting the dilute suspensions collected in the field into the concentrated suspensions required for SediGraph analysis. In the procedure used, the suspended-sediment sample remained wet frcm the time it was collected to the time it was analyzed. The two subsamples of about 2-12 L of water and sediment (finer than $63 \mu \mathrm{m}$ ) that were taken from the $A$ and $B$ churn splitters on the research vesse' were combined in polyethylene carboys to which $10-15 \mathrm{~mL}$ of chloroform was added to retard onganic growth. The carboys from all sampling sites were transported to the USGS laboratory in Denver. Colorado, where the suspended sediment was allowed to settle undisturbed for at least 35 days. At the maximum vertical settling distance of $36 \mathrm{~cm}$ in the carboys, this time was sufficient for all particles coarser than about $0.5 \mu \mathrm{m}$ to settle (assuming Stokesian settling of quartz spherical particles of d'ensity $\left.2.65 \mathrm{gm} / \mathrm{cm}^{3}\right)$. After 35 days or longer, the supernatant water was siphoned from the carboys, and the settled sediment was transferred to $1-\mathrm{L}$ glass jars (maximum settling distance, $15 \mathrm{~cm}$ ) where it was allowed to settle for another 35 days or more. The supernatant was siphoned off once more, and the settled sediment was transferred to 250 -mL polyethylene bottles. 
All analyses of the size distribution of particles finer than $63 \mu \mathrm{m}$ were made in the ISGS sediment laboratory in lowa City, lowa, by the SediGraph method as described by Lara and Matthes (1986). Duplicate measurements were made on 10 percent of the SediGraph samples, and if the results did not agree within 5 percent for all size classes, the analysis was repeated (Matthes and others, 1992). After the particle-size distribution was determined, the sample was dried and weighed. The dry mass was compared with the mass of sediment calculated from the concentration of suspended sediment finer than $63 \mu \mathrm{m}$ determined by the filtering-and-weighing procedure (silt and clay column in tables 13-15) and the known volume (4-24 L) of river water from which the analyzed sample was allowed to settle. The differences between the calculated dry masses and the measured dry masses were assumed to represent the material finer than $0.5 \mu \mathrm{m}$ that remained in suspension and was siphoned from the carboys along with the supernatant water. These differences have been added to the mass of material finer than $1 \mu \mathrm{m}$ for purposes of calculating the percentages listed in tables 16-18. The amount of siphoned material finer than $0.5 \mu \mathrm{m}$ averaged 14 percent for the July-August 1991 cruise, 17 percent for the October-November 1991 cruise, and 9 percent for the April-May 1992 cruise. The largest proportions (greater than 20 percent) of siphoned material finer than $0.5 \mu \mathrm{m}$ were in general measured in samples collected from the reach of the Upper Mississippi River that is controlled by the navigation dams. The proportion of siphoned material finer than $0.5 \mu \mathrm{m}$ averaged about 8 percent for the fre?-flowing river below St. Louis, Missouri, in which there are no navigation dams. 

Table 16.-Particle-size distribution of suspended-sediment samples collected from tha
Mississippl River and some of its tributaries for July-August 1991 cruise

[Finer than $63 \mu \mathrm{m}$ was determined by SediGraph and coarser than $63 \mu \mathrm{m}$ was determined by visual-accumulation tube; $\mathrm{mg} / \mathrm{L}$, milligrams per liter; $\mu \mathrm{m}$, micrometers; Analysis was done by U.S. Geological Survey sediment laboratory in Iowa City, Iowa; --, not enough material to determine median diameter]

\begin{tabular}{|c|c|c|c|c|c|c|c|c|c|c|c|c|c|}
\hline \multirow{2}{*}{$\begin{array}{l}\text { Date } \\
1991\end{array}$} & \multirow{2}{*}{ Site name } & \multirow{2}{*}{$\begin{array}{c}\text { Total } \\
\text { suspended- } \\
\text { sediment } \\
\text { concentration } \\
\text { (mg/L) }\end{array}$} & \multicolumn{10}{|c|}{ Percent finer than indicated slze ( $\mu \mathrm{m})$} & \multirow{2}{*}{$\begin{array}{c}\text { Median } \\
\text { diame*ar } \\
(\mu \mathrm{r})\end{array}$} \\
\hline & & & 1 & 2 & 4 & 8 & 16 & 32 & 63 & 125 & 250 & 500 & \\
\hline $7-05$ & $\begin{array}{l}\text { Mississippi R. above } \\
\text { St. Anthony Falls, Minn. }\end{array}$ & 32 & 54 & 61 & 70 & 79 & 85 & 91 & 95 & 97 & 99 & 100 & $<1$ \\
\hline $7-06$ & $\begin{array}{l}\text { Minnesota R. at } \\
\text { Mile 3.5, Minn. }\end{array}$ & 174 & 26 & 31 & 38 & 46 & 52 & 57 & 62 & 82 & 99 & 100 & 13 \\
\hline 7.08 & $\begin{array}{l}\text { Mississippi R. at } \\
\text { Hastings, Minn. }\end{array}$ & 82 & \multicolumn{10}{|c|}{ Not enough material was collected for size analysis. } & -- \\
\hline $7-10$ & $\begin{array}{l}\text { Mississippi R. near } \\
\text { Pepin, Wis. }\end{array}$ & 9 & \multicolumn{10}{|c|}{ Not enough material was collected for size analysis. } & -- \\
\hline $7-12$ & $\begin{array}{l}\text { Mississippi R. at } \\
\text { Trempealeau, Wis. }\end{array}$ & 28 & \multicolumn{10}{|c|}{ Not enough material was collected for size analysis. } & -- \\
\hline $7-15$ & $\begin{array}{l}\text { Mississippi R. below } \\
\text { Lock and Dam 9. Wis. }\end{array}$ & 73 & 47 & 54 & 64 & 75 & 86 & 98 & 100 & & & & 1 \\
\hline $7-18$ & $\begin{array}{l}\text { Mississippi R. at } \\
\text { Clinton, lowa }\end{array}$ & 66 & 48 & 56 & 65 & 76 & 87 & 98 & 100 & & & & 1 \\
\hline $7-21$ & $\begin{array}{l}\text { Mississippi R. at } \\
\text { Keokuk, lowa }\end{array}$ & 45 & 57 & 65 & 73 & 81 & 87 & 98 & 100 & & & & $<1$ \\
\hline $7-24$ & $\begin{array}{l}\text { Mississippi R. near } \\
\text { Winfield, Mo. }\end{array}$ & 74 & 48 & 54 & 61 & 70 & 84 & 96 & 100 & & & & 1 \\
\hline $7-25$ & $\begin{array}{l}\text { Illinois R, at } \\
\text { Hardin, Ill. }\end{array}$ & 47 & \multicolumn{10}{|c|}{ Not enough material was collected for size analysis. } & -- \\
\hline $7-27$ & $\begin{array}{l}\text { Missouri R. at } \\
\text { St. Charles, Mo. }\end{array}$ & 140 & 35 & 41 & 46 & 53 & 62 & 74 & 78 & 83 & 98 & 100 & 6 \\
\hline 7.29 & $\begin{array}{l}\text { Mississippi R. at } \\
\text { Thebes, Ill. }\end{array}$ & 106 & 40 & 46 & 51 & 59 & 68 & 77 & 79 & 82 & 98 & 100 & 4 \\
\hline $7-30$ & $\begin{array}{l}\text { Ohio R, at } \\
\text { Olmsted, Ill. }\end{array}$ & 22 & \multicolumn{10}{|c|}{ Not enough material was collected for size analysis. } & -- \\
\hline $8-03$ & $\begin{array}{l}\text { Mississippi R. below } \\
\text { Vicksburg, Miss. }\end{array}$ & 111 & 45 & 53 & 60 & 70 & 82 & 95 & 98 & 99 & 100 & & 2 \\
\hline $8-05$ & $\begin{array}{l}\text { Mississippi R. near } \\
\text { St. Francisville, La. }\end{array}$ & 116 & 47 & 54 & 63 & 72 & 82 & 92 & 98 & 99 & 100 & & 1 \\
\hline 8-07 & $\begin{array}{c}\text { Mississippi R. below } \\
\text { Belle Chasse, La. }\end{array}$ & 42 & 68 & 79 & 89 & 95 & 99 & 99 & 100 & & & & $<1$ \\
\hline
\end{tabular}

\footnotetext{
' Total suspended-sediment concentration (sand and fine material) is the average of composite $A$ and $B$ values in table 13 .

${ }^{2}$ Median diameters greater than $1.0 \mu \mathrm{m}$ computed by linear interpolation; those less than $1.0 \mu \mathrm{m}$ by linear extrapolation.

${ }^{3}$ Water velocities were too slow for collecting a representative depth-integrated sample, so that a subsample (for size analysis) was taken from the discharge-weighted pumped sample. Under such low-velocity conditions, concentrations of suspended sediment coarser than $63 \mu \mathrm{m}$ are presumed to be virtually zero.
} 
[Finer than $63 \mu \mathrm{m}$ was determined by SediGraph and coarser than $63 \mu \mathrm{m}$ was determined

by visual-accumulation tube; $\mathrm{mg} / \mathrm{L}$, milligrams per liter;

$\mu \mathrm{m}$, micrometers; Analysis was done by U.S. Geological Survey sediment labcratory in Iowa City, Iowa; --, not enough material to determine median diametex]

\begin{tabular}{|c|c|c|c|c|c|c|c|c|c|c|c|c|c|}
\hline \multirow{2}{*}{$\begin{array}{l}\text { Date } \\
1991\end{array}$} & \multirow{2}{*}{ Site name } & \multirow{2}{*}{$\begin{array}{l}\text { Total } \\
\text { suspended- } \\
\text { sediment } \\
\text { concentration } \\
(\mathrm{mg} /)^{1}\end{array}$} & \multicolumn{10}{|c|}{ Percent finer than indicated size $(\mu \mathrm{m})$} & \multirow{2}{*}{$\begin{array}{c}\text { Median } \\
\text { diameter } \\
(\mu \mathrm{m})\end{array}$} \\
\hline & & & 1 & 2 & 4 & 8 & 16 & 32 & 63 & 125 & 250 & 500 & \\
\hline 10-07 & $\begin{array}{l}\text { Mississippi R. above } \\
\text { St. Anthony Falls, Minn. }\end{array}$ & 12 & 70 & 74 & 80 & 91 & 99 & 100 & & & & & 0.6 \\
\hline $10-08$ & $\begin{array}{l}\text { Minnesota R. at } \\
\text { Mile 3.5, Minn. }\end{array}$ & 62 & 42 & 49 & 65 & 76 & 81 & 99 & 100 & & & & 2 \\
\hline $10-10$ & $\begin{array}{l}\text { Mississippi R. at } \\
\text { Hastings, Minn. }\end{array}$ & 45 & 60 & 71 & 82 & 92 & 98 & 98 & 100 & & & & $<1$ \\
\hline $10-13$ & $\begin{array}{l}\text { Mississippi R. near } \\
\text { Pepin, Wis. }\end{array}$ & 9 & \multicolumn{10}{|c|}{ Not enough material was collected for size analysis. } & -- \\
\hline $10-15$ & $\begin{array}{l}\text { Mississippi R. at } \\
\text { Trempealeau, Wis. }{ }^{3}\end{array}$ & 12 & 65 & 76 & 84 & 90 & 94 & 97 & 100 & & & & $<1$ \\
\hline $10-18$ & $\begin{array}{l}\text { Mississippi R. below } \\
\text { Lock and Dam 9, Wis. }{ }^{3}\end{array}$ & 28 & 58 & 71 & 84 & 94 & 99 & 99 & 100 & & & & $<1$ \\
\hline $10-22$ & $\begin{array}{l}\text { Mississippi R. at } \\
\text { Clinton, lowa }{ }^{3}\end{array}$ & 31 & 70 & 81 & 91 & 98 & 99 & 99 & 100 & & & & $<1$ \\
\hline 10-27 & $\begin{array}{l}\text { Mississippi R. at } \\
\text { Keokuk, lowa }{ }^{3}\end{array}$ & 32 & 62 & 76 & 87 & 94 & 98 & 98 & 100 & & & & $<1$ \\
\hline $10-30$ & $\begin{array}{l}\text { Mississippi R. near } \\
\text { Winfield, Mo. }\end{array}$ & 36 & 63 & 74 & 85 & 94 & 98 & 99 & 100 & & & & $<1$ \\
\hline 10-31 & $\begin{array}{l}\text { Illinois R. at } \\
\text { Hardin, } \text { Ill. }^{3}\end{array}$ & 143 & 40 & 53 & 68 & 83 & 94 & 99 & 99 & 100 & & & 2 \\
\hline $11-03$ & $\begin{array}{l}\text { Missouri R. at } \\
\text { St. Charles, Mo. }\end{array}$ & 306 & 21 & 26 & 33 & 39 & 47 & 52 & 54 & 58 & 94 & 100 & 26 \\
\hline $11-05$ & $\begin{array}{l}\text { Mississippi R. at } \\
\text { Thebes, 1ll. }\end{array}$ & 140 & 28 & 34 & 40 & 46 & 51 & 56 & 58 & 64 & 90 & 100 & 14 \\
\hline $11-06$ & $\begin{array}{l}\text { Ohio R. at } \\
\text { Olmsted. Itl. }{ }^{3}\end{array}$ & 20 & 51 & 60 & 71 & 81 & 89 & 95 & 97 & 98 & 99 & 100 & 1 \\
\hline $11-09$ & $\begin{array}{l}\text { Mississippi R. below } \\
\text { Vicksburg, Miss. }\end{array}$ & 182 & 40 & 48 & 55 & 64 & 73 & 82 & 90 & 96 & 99 & 100 & 3 \\
\hline $11-11$ & $\begin{array}{l}\text { Mississippi R. near } \\
\text { St. Francisville, La. }\end{array}$ & 209 & 38 & 46 & 53 & 62 & 71 & 80 & 85 & 93 & 100 & & 3 \\
\hline $11-13$ & $\begin{array}{l}\text { Mississippi R. below } \\
\text { Belle Chasse, La. }{ }^{4}\end{array}$ & 142 & 51 & 63 & 77 & 90 & 96 & 99 & 100 & & & & 1 \\
\hline $11-13$ & $\begin{array}{l}\text { Mississippi R. below } \\
\text { Belle Chasse, La. }{ }^{5}\end{array}$ & 112 & 58 & 68 & 81 & 92 & 98 & 100 & & & & & $<1$ \\
\hline
\end{tabular}

${ }^{1}$ Total suspended-sediment concentration (sand and fine material) is the average of composite $A$ and $B$ values in table 14

${ }^{2}$ Median diameters greater than $1.0 \mathrm{~mm}$ were computed by linear interpolation; those less than $\mathrm{I} .0 \mathrm{~mm}$ by linear extrapolation.

${ }^{3}$ Water velocities were too slow for collecting a representative depth-integrated sample, so that a subsample (for size ana'ysis) was taken from the discharge-weighted pumped sample. Under such low-velocity conditions. concentrations of suspended sediment coarser than 63 mm are presumed to be virtually zero.

${ }^{4}$ Depth-integrated sample.

${ }^{5}$ Discharge-weighted pumped sample. 


\section{Table 18.--Particie-size distribution of suspended-sediment samples coilected from th? Mississippi River and some of its tributaries for April-May 1992 cruise}

[Finer than $63 \mu \mathrm{m}$ was determined by SediGraph and coarser than $63 \mu \mathrm{m}$ was determined by visual-accumulation tube; $\mathrm{mg} / \mathrm{L}$, milligrams per liter; $\mu \mathrm{m}$, micrometers; Analysis was done by U.S. Geological Survey sediment laboratory in Iowa City, Iowa; --, not enough material to determine median diameter]

\begin{tabular}{|c|c|c|c|c|c|c|c|c|c|c|c|c|c|}
\hline \multirow{2}{*}{$\begin{array}{l}\text { Date } \\
1992\end{array}$} & \multirow{2}{*}{ Site name } & \multirow{2}{*}{$\begin{array}{c}\text { Total } \\
\text { suspended- } \\
\text { sediment } \\
\text { concentration } \\
\text { (mg/L)' }\end{array}$} & \multicolumn{10}{|c|}{ Percent finer than indicated size $(\mu \mathrm{m})$} & \multirow{2}{*}{$\begin{array}{c}\text { Median } \\
\text { diameter } \\
\text { ( } \mu \mathrm{r} \text { ) }\end{array}$} \\
\hline & & & 1 & 2 & 4 & 8 & 16 & 32 & 63 & 125 & 250 & 500 & \\
\hline $4-06$ & $\begin{array}{l}\text { Mississippi R. above } \\
\text { St. Anthony Falls, Minn. }\end{array}$ & 13 & \multicolumn{5}{|c|}{$\begin{array}{l}\text { Not enough material was col- } \\
\text { lected for size analysis. }\end{array}$} & 91 & 92 & 97 & \multicolumn{2}{|l|}{100} & - \\
\hline $4-08$ & $\begin{array}{l}\text { Minnesota } R \text {. at } \\
\text { Mile 3.5, Minn. }\end{array}$ & 106 & 24 & 32 & 42 & 54 & 66 & 84 & 94 & 99 & \multicolumn{2}{|l|}{100} & 7 \\
\hline 4-10 & $\begin{array}{l}\text { Mississippi R. at } \\
\text { Hastings, Minn. }\end{array}$ & 36 & 50 & 61 & 74 & 87 & 96 & \multicolumn{5}{|l|}{100} & 1 \\
\hline $4-12$ & $\begin{array}{l}\text { Mississippi R. near } \\
\text { Pepin, Wis. }{ }^{3}\end{array}$ & 12 & \multicolumn{10}{|c|}{ Not enough material was collected for size analysis. } & -- \\
\hline $4-14$ & $\begin{array}{l}\text { Mississippi R. at } \\
\text { Trempealeau, Wis. }\end{array}$ & 14 & \multicolumn{10}{|c|}{ Not enough material was collected for size analysis. } & -- \\
\hline $4-17$ & $\begin{array}{l}\text { Mississippi R. below } \\
\text { Lock and Dam 9, Wis. }\end{array}$ & 24 & 57 & 65 & 71 & 82 & 91 & 97 & 99 & \multirow{2}{*}{\multicolumn{3}{|c|}{100}} & $<1$ \\
\hline $4-19$ & $\begin{array}{r}\text { Mississippi R. at } \\
\text { Clinton, lowa }\end{array}$ & 40 & 55 & 63 & 71 & 81 & 90 & 97 & 100 & & & & $<1$ \\
\hline $4-23$ & $\begin{array}{r}\text { Mississippi R. at } \\
\text { Keokuk, lowa }\end{array}$ & 299 & 33 & 46 & 60 & 74 & 84 & 98 & 100 & & & & 3 \\
\hline $4-26$ & $\begin{array}{l}\text { Mississippi R. near } \\
\text { Winfield, Mo. }\end{array}$ & 308 & 36 & 48 & 59 & 71 & 84 & 94 & 96 & 98 & 99 & 100 & 2 \\
\hline $4-27$ & $\begin{array}{l}\text { Itlinois R. at } \\
\text { Hardin, III. }\end{array}$ & 230 & 42 & 55 & 70 & 85 & 95 & 99 & 100 & & & & 2 \\
\hline $4-29$ & $\begin{array}{l}\text { Missouri R. at } \\
\text { St. Charles, Mo. }\end{array}$ & 1,360 & 24 & 34 & 43 & 54 & 67 & 83 & 88 & 92 & 98 & 100 & 7 \\
\hline $5-01$ & $\begin{array}{l}\text { Mississippi R. at } \\
\text { Thebes, IIl. }\end{array}$ & 701 & 27 & 38 & 49 & 59 & 72 & 83 & 86 & 88 & 99 & 100 & 4 \\
\hline $5-03$ & $\begin{array}{l}\text { Ohio R. at } \\
\text { Olmsted, } 11 l^{3}\end{array}$ & 67 & 41 & 54 & 69 & 84 & 95 & 98 & 100 & & & & 2 \\
\hline $5-06$ & $\begin{array}{l}\text { Mississippi R. below } \\
\text { Vicksburg, Miss. }\end{array}$ & 358 & 20 & 34 & 48 & 60 & 72 & 82 & 84 & 87 & 97 & 100 & 5 \\
\hline $5-08$ & $\begin{array}{l}\text { Mississippi R. near } \\
\text { St. Francisville, La. }\end{array}$ & 335 & 25 & 38 & 52 & 64 & 74 & 84 & 90 & 94 & 100 & & 4 \\
\hline $5-10$ & $\begin{array}{c}\text { Mississippi R. below } \\
\text { Belle Chasse, La. }\end{array}$ & 311 & 36 & 49 & 64 & 76 & 88 & 96 & 98 & 99 & 100 & & 2 \\
\hline
\end{tabular}




\section{SUMMARY}

The average (all sites and all cruises) median diameter of the bed sediments in the navigation lock and dam reach of the Mississippi River was $0.70 \mathrm{~mm}$ and the average median diamete- was $0.40 \mathrm{~mm}$ in the free-flowing reach of the Mississippi River downstream from St. Louis, Missouri. Suspendedsediment samples from the Mississippi River were collected from water discharges ranging from $220 \mathrm{~m}^{3} / \mathrm{s}$ above St. Anthony Falls, Minnesota, in October 1991 to 21,700 m³ $/ \mathrm{s}$ below Vicksburg, Mississippi, in May 1992; in mean water depths ranging from $2.5 \mathrm{~m}$ above St. Anthony Falls, Minnesota, in July 1991 to $20.6 \mathrm{~m}$ below Belle Chasse, Louisiana, in May 1992; and in cross-sectional mean velocities ranging from $0.30 \mathrm{~m} / \mathrm{s}$ below Belle Chasse, in August 1991 and $0.30 \mathrm{~m} / \mathrm{s}$ near Pepin, Wisconsin in October 1991, to $1.66 \mathrm{~m} / \mathrm{s}$ at Thebes, Illinois, in April 1992. The average (all sites and all cruises) total suspended-sediment concentration in the navigation lock and dam reach of the Mississippi River was $34 \mathrm{mg} / \mathrm{L}$, and 55 percent of this suspended sediment was less than $1 \mu \mathrm{m}$ in size. However, in April 1992 at Keokuk, Iowa, and near Winfield, Missouri, heavy runoff from the lowa and Des Moines Rivers increased the total suspended-sediment concentrations to about $300 \mathrm{mg} / \mathrm{L}$, of which about 34 percent was less than $1 \mu \mathrm{m}$ in size. In the free-flowing reach of the Mississippi River downstrean from St. Louis, Missouri, the average (all sites and all cruises) total suspended-sediment concentration was $229 \mathrm{mg} / \mathrm{L}$, of which 40 percent was less than $1 \mu \mathrm{m}$ in size. 


\section{REFERENCES CITED}

Chow, V.T., 1959, Open-channel hydraulics: New York, McGraw-Hill, p. 680.

Guy, H.P., 1969, Laboratory theory and methods for sediment analysis: U.S. Geolngical Survey Techniques of Water-Resources Investigations, book 5, chap. C1, 58 p.

Guy, H.P., and Norman, V.W., 1970, Field methods for measurement of fluvial sediment: U.S. Geological Survey Techniques of Water-Resources Investigations, book 3, chap. C2, 59 p.

Keown, M.P., Dardeau, E.A., Jr., Causey, E.M., 1981, Characterization of the suspendedsediment regime and bed-material gradation of the Mississippi River Basin: U.S. Army Engineer Waterways Experiment Station Potamology Program (P-1) Report 1, v. 1 and v. 2,874 p.

Lara, O.G., and Matthes, W.J., Jr., 1986, The SediGraph as an alternative method to the pipet, in Proceedings of the Fourth Federal Interagency Sedimentation Conference 1986: Interagency Advisory Committee on Water Data, Subcommittee on Sedimentation, p. 1-1 to $1-12$.

Leenheer, J.A., Meade, R.H., Taylor, H.E., and Pereira, W.E., 1989, Sampling, fractionation, and dewatering of suspended sediment from the Mississippi River for geochemical and trace-contaminant analysis, in Mallard, G.E., and Ragone, S.E., eds., U.S. Geolcoical Survey Toxic Substances Hydrology Program--Proceedings of the Technical Meeting, Phoenix, Arizona, September 26-30, 1988: U.S. Geological Survey Water-Resources Investigations Report 88-4220, p. 501-511.

Matthes, W.J., Jr., Sholar, C.J., and George, J.R., 1992, Quality-assurance plan for the analysis of fluvial sediment by laboratories of the U.S. Geological Survey: U.S. Geological Survey Open-File Report 91-467, 31 p.

Meade, R.H., and Stevens, H.H., Jr., 1990, Strategies and equipment for sampling suspended sediment and associated toxic chemicals in large rivers--with emphasis on the Mississippi River: Science of the Total Environment, v. 97/98, p. 125-135.

Moody, J.A., 1993, Evaluation of the Langrangian scheme for sampling the Mississippi River during 1987-90: U.S. Geological Survey Water-Resources Investigations

Report 93-4042, 31 p.

Moody, J.A., and Meade, R.H., 1992, Hydrologic and sedimentologic data collected during three cruises at low water on the Mississippi River and some of its tributaries, July 1987June 1988: U.S. Geological Survey Open-File Report 91-485, 143 p.

Moody, J.A., and Meade, R.H., 1993, Hydrologic and sedimentologic data collected during four cruises at high water on the Mississippi River and some of its tributaries, March 1989-June 1990: U.S. Geological Survey Open-File Report 92-651, 227 p.

Moody, J.A., and Meade, R.H., 1994, Evaluation of the method of collecting suspended sediment from large rivers by discharge-weighted pumping and separating it by continuousflow centrifugation: Hydrological Processes, v. 8, p. 513-530. 
Moody, J.A., and Troutman, B.M., 1992, Evaluation of the depth-integraticn method of measuring water discharge in large rivers: Journal of Hydrology, v. 135, p. 201-236.

Nordin, C.F., Jr., Cranston, C.C., and Mejía-B.,A., 1983, New technology for measuring water and suspended-sediment discharge of large rivers, in Proceedings of the International Symposium on River Sedimentation, 2d, Nanjing: Beijing, Water Resources and Electric Power Press, p. 1145-1158.

Pereira, W.E., Moody, J.A., Hostettler, F.D., Rostad, C.E., and Leiker, T.J., 1995, Concentrations and mass transport of pesticides and organic contaminants in the Mississippi River and some of its tributaries, 1987-89 and 1991-92: U.S. Geological Survey Open-File Report 94-376.

Rees, T.F., Leenheer, J.A., and Ranville, J.F., 1991, Use of a single-bowl continuous-flow centrifuge for dewatering suspended sediments--effect on sediment physical and chemical characteristics: Hydrological Processes, v. 5, p. 201-214.

Richey, J.E., Meade, R.H., Salati, Eneas, Devol, A.H., Nordin, C.F., Jr., and dos Santos, Umberto, 1986, Water discharge and suspended sediment concentrations ir the Amazon River, 1982-1984: Water Resources Research, v. 22, no. 5, p. 756-764.

Sayre, W.W., and Caro-Cordero, R., 1977, Shore-attached thermal plumes in rivers, in Shen, H.W., ed., Modeling of Rivers: New York, John Wiley, chap. 15, p. 15-1 to 15-44.

Sium, Oghazghi, 1975, Transverse flow distribution in natural streams as influerced by crosssectional shape: M.S. Thesis, Ames, University of Iowa, 99 p.

Stallard, R.F., and Martin, D.A., 1989, Settling properties of suspended sediment from the Mississippi River [abs.], in Pederson, G.L., and Smith, M.M., compilers, U.S. Geological Survey Second National Symposium on Water Quality--Abstracts of the Technical Sessions, Orlando, Fla., November 12-17, 1989: U.S. Geological Survey Open-File Report 89-409, p. 96.

Stevens, H.H., Jr., Lutz, G.A., and Hubbell, D.W., 1980, Collapsible-bag suspended-sediment sampler: American Society of Civil Engineers Proceedings, Hydraulics Division Journal, v. 106, no. HY4, p. 611-616.

U.S. Army Corps of Engineers, [1983?], Missouri River navigation charts (Kansas City, Missouri to the mouth): Kansas City, Mo. [variously paged].

1986?], Navigation charts McClellan-Kerr Arkansas River Navigation Syst`m (Catoosa, Oklahoma to mouth of White River): Little Rock, Ark. [variously paged].

1987a, Ohio River navigation charts (Cairo, Illinois to Foster, Kentucky): Louisville, Ky., District [variously paged].

1987b, Charts of the Illinois waterway: Washington, D.C., U.S. Government Printing Office [variously paged].

[1989a?], 1989 Flood control and navigation maps of the Mississippi River (Cairo, Illinois to the Gulf of Mexico) (57th ed.): Memphis, Tenn. [variously paged]. 
[1989b?], Upper Mississippi River navigation charts: Rock Island, Ill. [variously paged].

U.S. Geological Survey, 1989, Water-resources data for Iowa, water years 1989: U.S. Geological Survey Water-Data Reports IA-89-1 to IA-92-1, 399 p.

___ 1990a, Water-resources data for Arkansas, water year 1989: U.S. Geological Survey $\mathrm{V}^{\prime}$ aterData Report AR-89-1, 581 p.

___1990b, Water-resources data for Illinois, water year 1989: U.S. Geological Survey h'aterData Reports IL-89-1, v.7, 387 p.

___1990c, Water-resources data for Missouri, water year 1989: U.S. Geological Survey V'aterData Reports MO-89-1, 354 p.

___ 1990d, Water-resources data for Minnesota, water year 1988: U.S. Geological Survey Water-Data Reports MN-88-1, 331 p.

___1990e, Water-resources data for Wisconsin, water year 1989: U.S. Geological Survey Water-Data Reports WI-89-1, 436 p. 


\section{TABULATED CRUISE DATA}

The data in this section are organized by individual cruises. In the listings of measurements made at each vertical: The $A$ and $B$ after the vertical number identifies the sample bottle used at that vertical, the $X$ preceding the vertical number indicates that an extra velocity mea surement was made but no sample was collected, and an $R$ indicates that the vertical was repeate $X$ because the velocity measurement was incorrect, the suspended-sediment collection bag leaked. or debris on the nozzle prevented the collection of a suitable sample. Verticals usually were occupied in numerical order. Exceptions owing to weather conditions or towboat traffic are noted in the REMARKS section of each sampling site listing. Inch-pound units are used in these listings for: (1) part of the name of a sampling site; (2) gage height, which is the elevation of the gege in feet above sea level (National Geodetic Vertical Datum of 1929) and which will serve as a ref rence for future cruises; (3) the name of a sampling weight; and (4) the designation of the nozzle size used to sample suspended sediment. In the current meter equation the * represents multiplination.

The following abbreviations are used in the tabulated cruise data and are listed below.

$$
\begin{aligned}
& \text { SUSP }=\text { type of suspension } \\
& \mathrm{pH}=\text { surface } \mathrm{pH} \text { of the river water } \\
& \text { LEW = left edge of water } \\
& \text { REW = right edge of water } \\
& \operatorname{Rev} / \mathrm{s}=\text { revolutions per second } \\
& \text { SAFU = St. Anthony Falls Upper Pool } \\
& \mathrm{TW}=\text { tailwater } \\
& \text {-- = no sample was collected or no measurement was made } \\
& \mathrm{ft}=\text { feet } \\
& \mathrm{lb}=\text { pound } \\
& \mathrm{cm}=\text { centimeter } \\
& \mathrm{cm} / \mathrm{s}=\text { centimeter per second } \\
& { }^{\circ} \mathrm{C}=\text { degree Celsius } \\
& \mathrm{L}=\text { liter } \\
& \mu \mathrm{S} / \mathrm{cm}=\text { microsiemens per centimeter at } 25 \text { degrees Celsius } \\
& <63 \mu \mathrm{m}=\text { finer than } 63 \text { micrometers } \\
& >63 \mu \mathrm{m}=\text { greater than } 63 \text { micrometers } \\
& \mathrm{m} / \mathrm{s}=\text { meters per second } \\
& \mathrm{s}=\text { second }
\end{aligned}
$$




\title{
DATA LIST
}

\author{
FOR
}

\section{JULY-AUGUST 1991 CRUISE}


SITE: Mississippi River above St. Anthony Falls, Minn.-Mile 858.3

07-05-91

PARTY: Moody, Garbarino, Antweiler, and Simoneaux

GAGE HEIGHT @ Anoka: 810.63 ft GAGE HEIGHT @ SAFU Pool: $800.48 \mathrm{ft}$

RIVER SLOPE: $178 \times 10^{-6}$

SUSP. Bag sampler and 150 -lb weight

PRICE AA CURRENT METER No: W-223906 DATE RATED: 06-91

CURRENT METER EQUATION: $V(\mathrm{~m} / \mathrm{s})=\operatorname{Rev} / \mathrm{s}^{*} 0.670+0.010$

REMARKS:

Anchored at five verticals, used 5/16-inch nozzle and transit rates that ranged from 5.0 to $8.0 \mathrm{~cm} / \mathrm{s}$.

Section was about $100 \mathrm{~m}$ downriver from the Minneapolis Water Works intake.

\begin{tabular}{|c|c|c|c|c|c|c|c|}
\hline Vertical & $\begin{array}{l}\text { Distance } \\
\text { from } \\
\text { LEW } \\
\text { (m) }\end{array}$ & $\begin{array}{l}\text { Depth } \\
\text { (m) }\end{array}$ & $\begin{array}{l}\text { Mean } \\
\text { velocity } \\
(\mathbf{m} / \mathbf{s})\end{array}$ & $\begin{array}{c}\text { Discharge } \\
\mathrm{m}^{3} / \mathrm{s}\end{array}$ & $\begin{array}{c}\text { Temperature } \\
\left({ }^{\circ} \mathrm{C}\right)\end{array}$ & pH & $\begin{array}{c}\text { Specific } \\
\text { conductance } \\
(\mu \mathrm{S} / \mathrm{cm})\end{array}$ \\
\hline LEW & 0 & 0.0 & & & & & \\
\hline 01A,B & 44 & 3.1 & 0.97 & 86 & - & - & - \\
\hline 02A,B & 57 & 3.7 & 0.91 & 59 & - & - & - \\
\hline 03A,B & 79 & 4.0 & 0.92 & 79 & -- & - & $\cdots$ \\
\hline $\mathrm{X} 03$ & 100 & 3.3 & 0.96 & 46 & - & -- & -- \\
\hline 04A,B & 108 & 3.1 & 0.98 & 40 & - & -- & - \\
\hline 05A,B & 126 & 3.6 & 0.90 & 39 & - & -- & - \\
\hline X04 & 132 & 3.6 & 0.90 & 120 & & & \\
\hline REW & 200 & 0.0 & 0.00 & & & & \\
\hline MEANS & & 2.5 & 0.93 & & & & \\
\hline TOTAL & 200 & & & 467 & & & \\
\hline
\end{tabular}




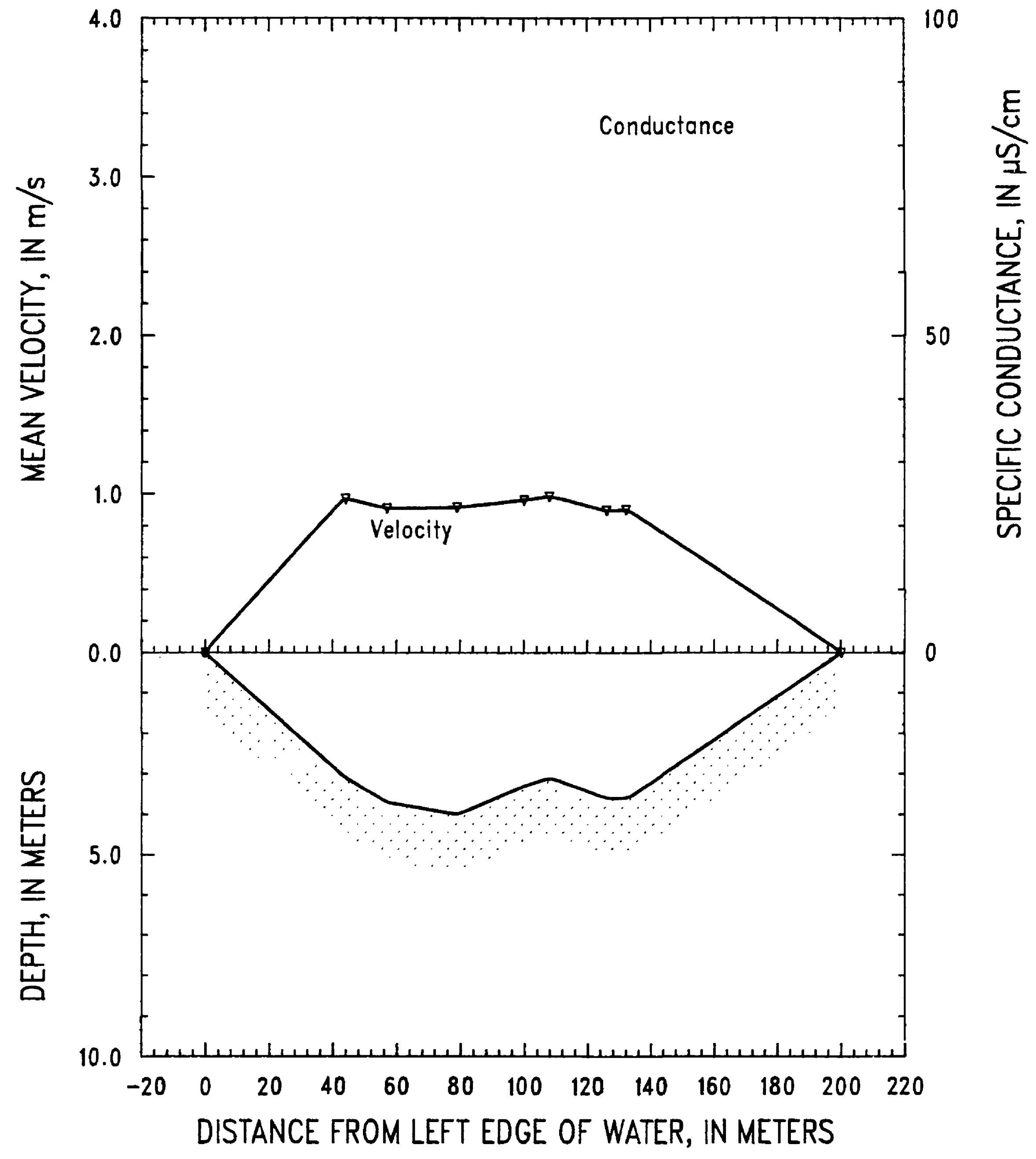

Figure 7. Mississippi River above St. Anthony. Falls, Minnesota, on July 5, 1991. 
SITE: Minnesota River at Mile 3.5, Minn.

07-06-91

PARTY: Moody, Garbarino, Antweiler, and Simoneaux

GAGE HEIGHT @ Savage: $699.46 \mathrm{ft}$ GAGE HEIGHT @ Pool 2: $686.68 \mathrm{ft}$

RIVER SLOPE: $55 \times 10^{-6}$

SUSP. Bag sampler and 150-lb weight

PRICE AA CURRENT METER No: W-223906 DATE RATED: 06-91

CURRENT METER EQUATION: $\mathrm{V}(\mathrm{m} / \mathrm{s})=\operatorname{Rev} / \mathrm{s}^{*} 0.670+0.010$

REMARKS:

Anchored at five verticals, used 5/16- and 1/4-inch nozzles and transit rates that ranged from 7.0 to $17.0 \mathrm{~cm} / \mathrm{s}$. Section was about $50 \mathrm{~m}$ upstream from the Metropolitan Waste Control Commission dock.

\begin{tabular}{|c|c|c|c|c|c|c|c|}
\hline Vertical & $\begin{array}{l}\text { Distance } \\
\text { from } \\
\text { LEW } \\
\text { (m) }\end{array}$ & $\begin{array}{l}\text { Depth } \\
\text { (m) }\end{array}$ & $\begin{array}{c}\text { Mean } \\
\text { velocity } \\
(\mathrm{m} / \mathrm{s})\end{array}$ & $\begin{array}{c}\text { Discharge } \\
\left(\mathrm{m}^{3} / \mathrm{s}\right)\end{array}$ & $\begin{array}{c}\text { Temperature } \\
\left({ }^{\circ} \mathrm{C}\right)\end{array}$ & pH & $\begin{array}{c}\text { Specific } \\
\text { conductance } \\
(\mu \mathrm{S} / \mathrm{cm})\end{array}$ \\
\hline$\overline{\text { LEW }}$ & 0 & 0.0 & 0.00 & & & & \\
\hline$X 05$ & 21 & 6.0 & 1.04 & 72 & - & -- & - \\
\hline 01A,B & 23 & 6.6 & 1.19 & 35 & 25.8 & 8.1 & 760 \\
\hline X06 & 30 & 6.9 & 1.15 & 55 & - & -- & - \\
\hline $02 \mathrm{~A}, \mathrm{~B}$ & 37 & 7.0 & 1.07 & 38 & 25.9 & 8.1 & 762 \\
\hline$X 04$ & 40 & 7.0 & 1.06 & 56 & - & - & -- \\
\hline 03A,B & 52 & 7.0 & 1.07 & 60 & 25.4 & 8.1 & 776 \\
\hline$X 01$ & 56 & 7.1 & 1.20 & 68 & -- & - & - \\
\hline $04 \mathrm{~A}, \mathrm{~B}$ & 68 & 6.8 & 1.11 & 91 & 26.3 & 8.2 & 761 \\
\hline $05 A, B$ & 80 & 6.1 & 1.01 & 49 & 25.2 & 8.2 & 775 \\
\hline$X 02$ & 84 & 5.8 & 0.89 & 75 & -- & - & - \\
\hline REW & 109 & 0.0 & 0.00 & & & & \\
\hline MEANS & & 5.2 & 1.06 & & & & \\
\hline TOTAL & 109 & & & 598 & & & \\
\hline
\end{tabular}




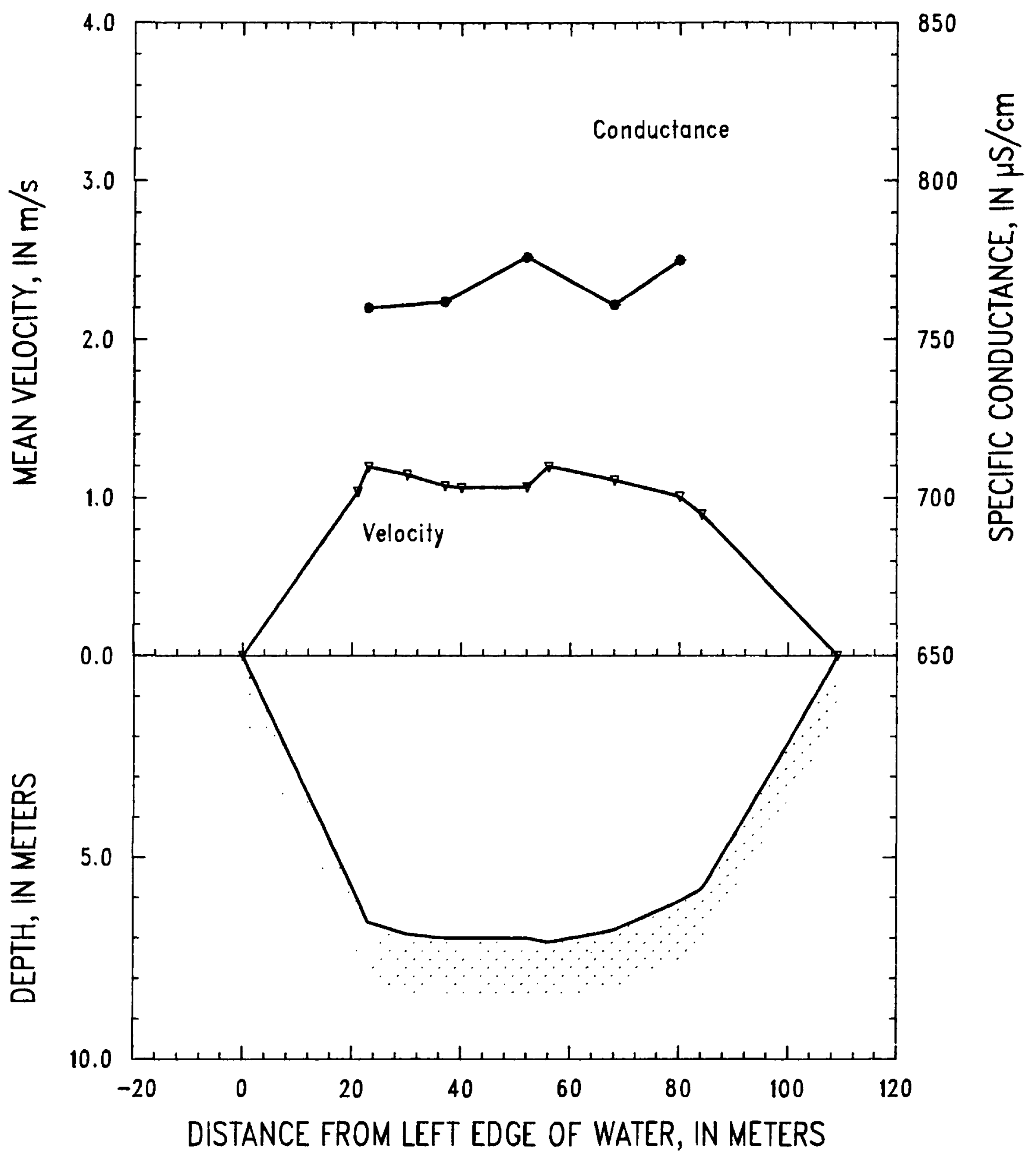

Figure 8. Minnesota River at Mile 3.5, Minnesota, on July 6, 1991. 
SITE: Mississippi River at Hastings, Minn.-Mile 812.2

07-08-91

PARTY: Moody, Garbarino, and Simoneaux

GAGE HEIGHT @ TW Pool 2: $681.28 \mathrm{ft}$ GAGE HEIGHT @ Pool 3: $674.99 \mathrm{ft}$

RIVER SLOPE: $66 \times 10^{-6}$

SUSP. Bag sampler and 150-lb weight

PRICE AA CURRENT METER No: W-223906 DATE RATED: 06-91

CURRENT METER EQUATION: $V(\mathrm{~m} / \mathrm{s})=\operatorname{Rev} / \mathrm{s}^{*} 0.670+0.010$

REMARKS:

Sampled eight verticals (equal-width increment) using a 5/16-inch nozzle and a transit rate of $11 \mathrm{~cm} / \mathrm{s}$.

\begin{tabular}{|c|c|c|c|c|c|c|c|}
\hline Vertical & $\begin{array}{c}\text { Distance } \\
\text { from } \\
\text { LEW } \\
\text { (m) }\end{array}$ & $\begin{array}{c}\text { Depth } \\
\text { (m) }\end{array}$ & $\begin{array}{c}\text { Mean } \\
\text { velocity } \\
(\mathrm{m} / \mathrm{s})\end{array}$ & $\begin{array}{c}\text { Discharge } \\
\left(\mathrm{m}^{3} / \mathrm{s}\right)\end{array}$ & $\begin{array}{c}\text { Temperature } \\
\left({ }^{\circ} \mathrm{C}\right)\end{array}$ & pH & $\begin{array}{c}\text { Specific } \\
\text { conductance } \\
(\mu \mathrm{S} / \mathrm{cm})\end{array}$ \\
\hline LEW & 0 & 0.0 & 0.00 & & & & \\
\hline $01 \mathrm{~A}, \mathrm{~B}$ & 31 & 9.0 & 0.79 & 157 & 24.3 & 7.8 & 647 \\
\hline$X 12$ & 44 & 8.3 & 0.90 & 86 & -- & - & -- \\
\hline $02 A, B$ & 54 & 8.0 & 0.86 & 124 & 24.5 & 7.7 & 644 \\
\hline 03A,B & 80 & 7.1 & 0.85 & 108 & 24.6 & 7.8 & 646 \\
\hline X03 & 90 & 7.1 & 0.84 & 83 & -- & -- & -- \\
\hline $04 A, B$ & 108 & 6.7 & 0.69 & 78 & 24.6 & 7.9 & 646 \\
\hline X04 & 124 & 6.2 & 0.82 & 61 & -- & - & -- \\
\hline 05A,B & 132 & 6.0 & 0.81 & 83 & 24.6 & 8.0 & 647 \\
\hline 06A,B & 158 & 5.2 & 0.69 & 50 & 24.7 & 8.0 & 647 \\
\hline X05 & 160 & 5.2 & 0.72 & 41 & -- & - & -- \\
\hline 07A,B & 180 & 4.9 & 0.58 & 35 & 24.5 & 8.0 & 648 \\
\hline X06 & 185 & 4.9 & 0.57 & 28 & -- & -- & - \\
\hline$\times 07$ & 200 & 4.0 & 0.43 & 15 & -- & - & -- \\
\hline 08A,B & 203 & 3.3 & 0.51 & 34 & 24.5 & 8.0 & 647 \\
\hline REW & 240 & 0.0 & 0.00 & & & & \\
\hline MEANS & & 5.4 & 0.76 & & & & \\
\hline TOTAL & 240 & & & 983 & & & \\
\hline
\end{tabular}




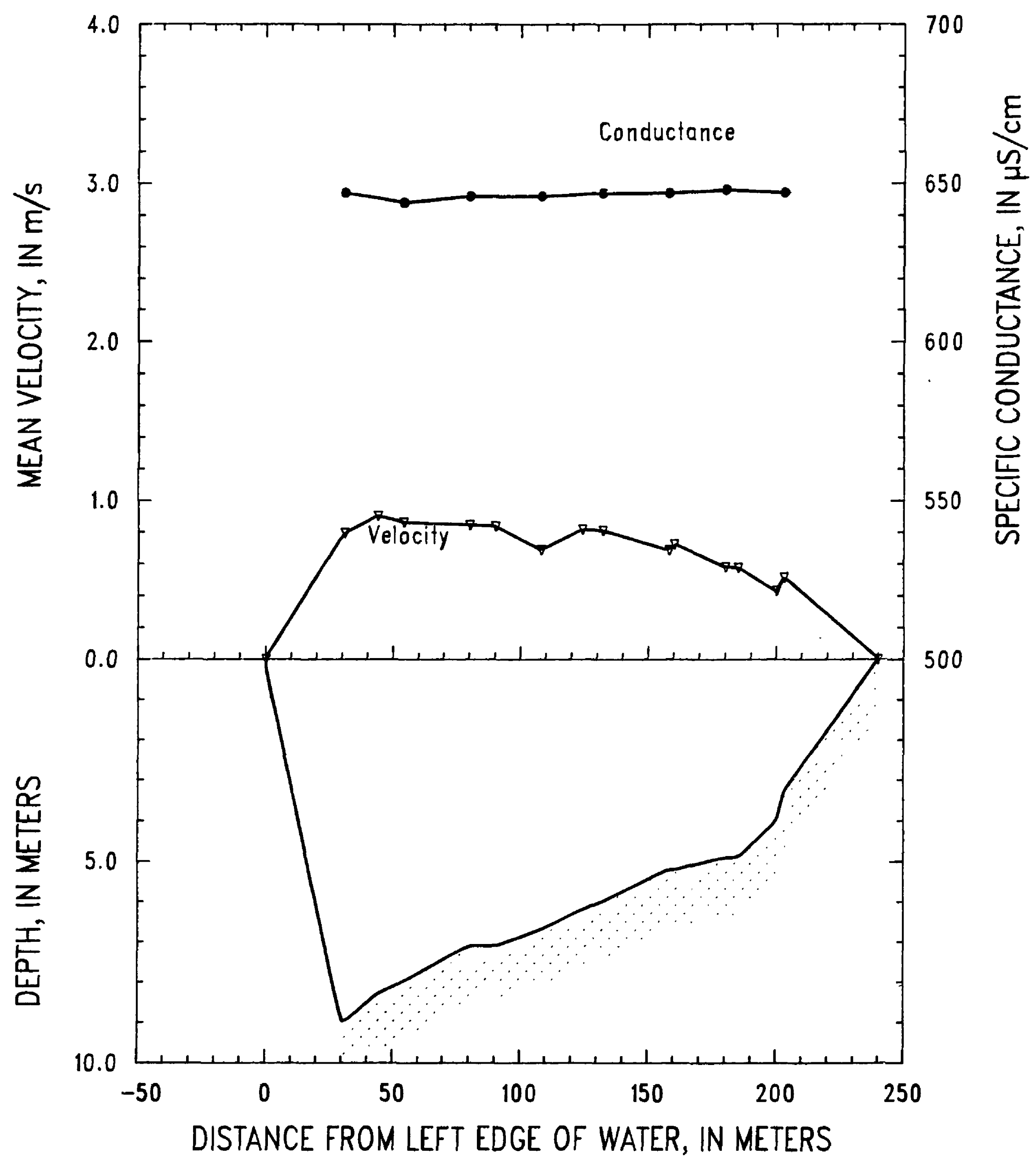

Figure 9. Mississippi River at Hastings, Minnesota, on July 8, 1991. 
SITE: Mississippi River near Pepin, Wis.-Mile 764.5

07-10-91

PARTY: Moody, Garbarino, and Simoneaux

GAGE HEIGHT @ Lake City: $671.81 \mathrm{ft}$ GAGE HEIGHT @ Wabasha: $669.60 \mathrm{ft}$

RIVER SLOPE: $34 \times 10^{-6}$

SUSP. Bag sampler and 150-lb weight

PRICE AA CURRENT METER No: W-223906 DATE RATED: 06-91

CURRENT METER EQUATION: $\mathrm{V}(\mathrm{m} / \mathrm{s})=\operatorname{Rev} / \mathrm{s}^{*} 0.670+0.010$

REMARKS:

Sampled 12 verticals (equal-discharge increment) using a 5/16-inch nozzle and variable transit rates ranging from 2 to $8.5 \mathrm{~cm} / \mathrm{s}$. Verticals sampled from 12 to 1 .

\begin{tabular}{|c|c|c|c|c|c|c|c|}
\hline Vertical & $\begin{array}{l}\text { Distance } \\
\text { from } \\
\text { LEW } \\
\text { (m) }\end{array}$ & $\begin{array}{l}\text { Depth } \\
\text { (m) }\end{array}$ & $\begin{array}{c}\text { Mean } \\
\text { velocity } \\
(\mathrm{m} / \mathrm{s})\end{array}$ & $\begin{array}{c}\text { Discharge } \\
\left(\mathrm{m}^{3} / \mathrm{s}\right)\end{array}$ & $\begin{array}{c}\text { Temperature } \\
\left({ }^{\circ} \mathrm{C}\right)\end{array}$ & pH & $\begin{array}{c}\text { Specific } \\
\text { conductance } \\
(\mu \mathrm{S} / \mathrm{cm})\end{array}$ \\
\hline LEW & 0 & 0.0 & 0.00 & & & & \\
\hline$\times 05$ & 65 & 3.6 & 0.55 & 71 & - & - & - \\
\hline $01 A, B$ & 72 & 3.7 & 0.61 & 67 & 26.8 & 8.2 & 549 \\
\hline O2B & 124 & 3.9 & 0.59 & 66 & - & -- & - \\
\hline $02 \mathrm{~A}$ & 129 & 3.9 & 0.59 & 21 & 26.1 & 8.1 & 550 \\
\hline X04 & 142 & 4.0 & 0.63 & 50 & - & - & - \\
\hline 03A,B & 169 & 4.0 & 0.58 & 71 & 26.1 & 8.0 & 549 \\
\hline $04 \mathrm{~A}$ & 203 & 3.9 & 0.64 & 50 & 25.3 & 7.8 & 551 \\
\hline 04B & 209 & 4.1 & 0.59 & 35 & -- & - & -- \\
\hline $\mathrm{X} 03$ & 232 & 3.4 & 0.68 & 36 & - & - & - \\
\hline 05A & 240 & 4.3 & 0.61 & 18 & 26.1 & 7.9 & 549 \\
\hline$\times 07$ & 246 & 4.2 & 0.71 & 16 & - & - & - \\
\hline 05B & 251 & 4.3 & 0.63 & 46 & - & - & -- \\
\hline 06A,B & 280 & 4.8 & 0.62 & 83 & 26.4 & 7.9 & 550 \\
\hline $07 A, B$ & 307 & 5.2 & 0.65 & 89 & 26.6 & 8.0 & 549 \\
\hline $\mathrm{X} 02$ & 333 & 6.0 & 0.66 & 63 & - & - & -- \\
\hline $08 \mathrm{~A}, \mathrm{~B}$ & 339 & 6.2 & 0.68 & 61 & 24.9 & 7.8 & 551 \\
\hline $09 \mathrm{~A}, \mathrm{~B}$ & 362 & 6.9 & 0.66 & 100 & 24.8 & 7.8 & 551 \\
\hline $10 \mathrm{~A}, \mathrm{~B}$ & 383 & 8.2 & 0.67 & 101 & 25.5 & 7.9 & 549 \\
\hline $11 \mathrm{~A}, \mathrm{~B}$ & 399 & 9.1 & 0.65 & 119 & 25.1 & 7.8 & 549 \\
\hline $12 \mathrm{~B}$ & 423 & 7.6 & 0.66 & 75 & - & - & - \\
\hline $12 \mathrm{~A}$ & 429 & 7.9 & 0.68 & 116 & 25.2 & 7.8 & 548 \\
\hline REW & 466 & 0.0 & 0.00 & & & & \\
\hline MEANS & & 4.6 & 0.63 & & & & \\
\hline TOTAL & 466 & & & 1,352 & & & \\
\hline
\end{tabular}

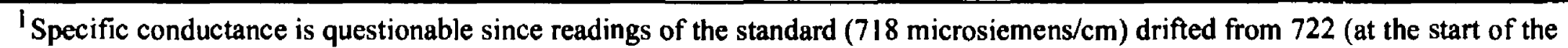
section) to 701 (at the end of the section). 


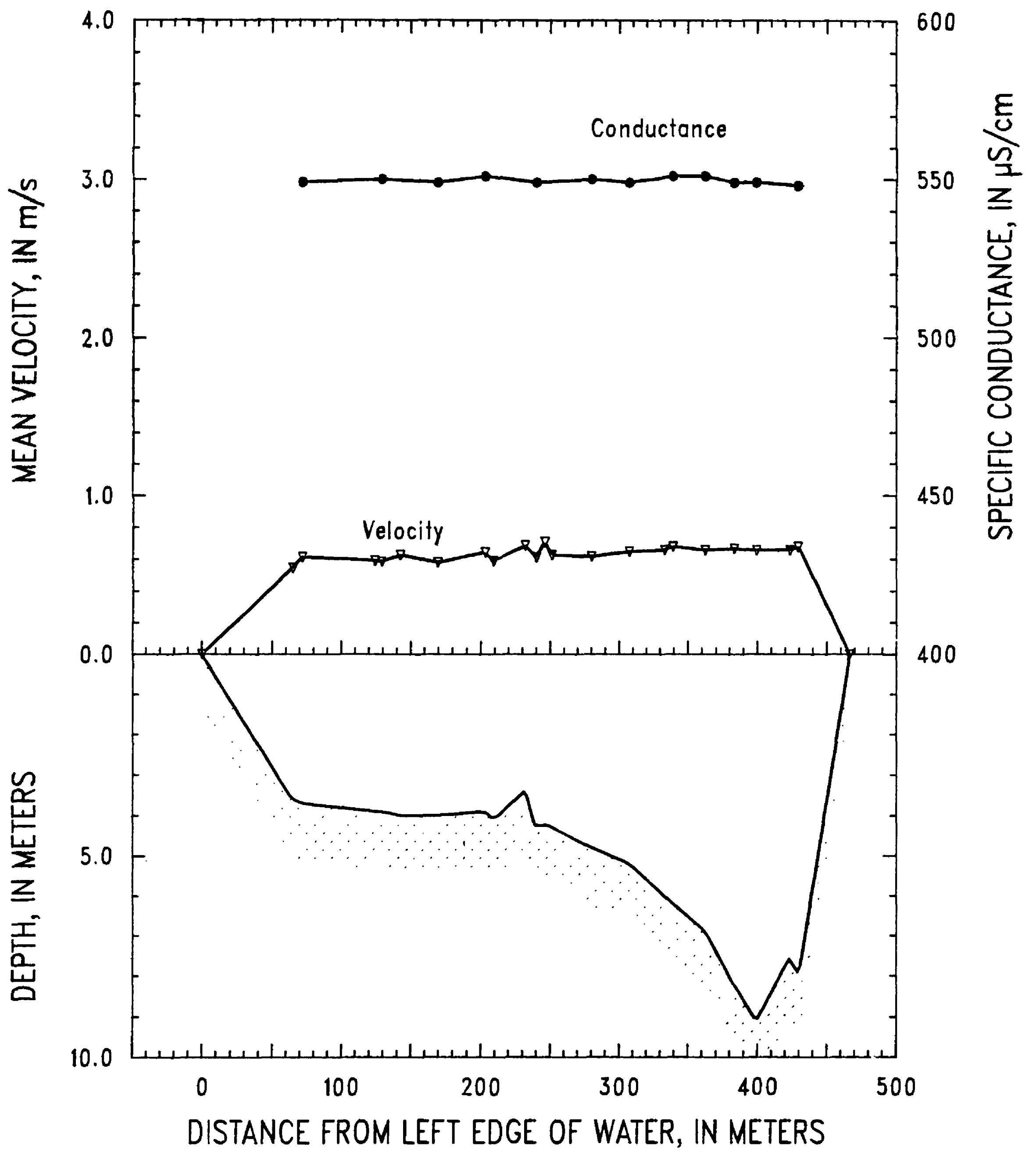

Figure 10. Mississippi River near Pepin, Wisconsin, on July 10, 1991. 
SITE: Mississippi River at Trempealeau, Wis.-Mile 713.8

$07-12-91$

PARTY: Moody, Roth, and Simoneaux

GAGE HEIGHT @ Pool 6 TW: 642.33 ft GAGE HEIGHT @ Pool 7: 639.03 ft

RIVER SLOPE: $57 \times 10^{-6}$

SUSP. Bag sampler and 150-lb weight

PRICE AA CURRENT METER No: W-223906 DATE RATED: 06-91

CURRENT METER EQUATION: $V(\mathrm{~m} / \mathrm{s})=\operatorname{Rev} / \mathrm{s}^{*} 0.670+0.010$

REMARKS:

Unmeasured flow through a shallow side channel was estimated at $50 \mathrm{~m}^{3} / \mathrm{s}$. Sampled eight verticals (equalwidth increment) using a 5/16-inch nozzle and transit rate of $7 \mathrm{~cm} / \mathrm{s}$. Discharge at Dam 6 was reported to be $1,625 \mathrm{~m}^{3} / \mathrm{s}$. Verticals sampled from 8 to 1 .

\begin{tabular}{|c|c|c|c|c|c|c|c|}
\hline Verticai & $\begin{array}{c}\text { Distance } \\
\text { from } \\
\text { LEW } \\
\text { (m) }\end{array}$ & $\begin{array}{l}\text { Depth } \\
\text { (m) }\end{array}$ & $\begin{array}{l}\text { Mean } \\
\text { velocity } \\
(\mathrm{m} / \mathrm{s})\end{array}$ & $\begin{array}{c}\text { Discharge } \\
\left(\mathrm{m}^{3} / \mathrm{s}\right)\end{array}$ & $\begin{array}{c}\text { Temperature } \\
\left({ }^{\circ} \mathrm{C}\right)\end{array}$ & pH & $\begin{array}{c}\text { Specific } \\
\text { conductance } \\
(\mu \mathrm{S} / \mathrm{cm})\end{array}$ \\
\hline LEW & 0 & 0.0 & 0.00 & & & & \\
\hline $01 \mathrm{~A}, \mathrm{~B}$ & 61 & 2.5 & 0.69 & 87 & 25.8 & 7.8 & 492 \\
\hline $02 \mathrm{~A}, \mathrm{~B}$ & 101 & 4.8 & 0.65 & 129 & 25.5 & 7.7 & 491 \\
\hline 03A,B & 144 & 5.1 & 0.68 & 159 & 25.8 & 7.8 & 497 \\
\hline $04 \mathrm{~A}, \mathrm{~B}$ & 193 & 4.1 & 0.75 & 113 & 25.0 & 7.8 & 501 \\
\hline X03 & 218 & 4.1 & 0.87 & 105 & -- & - & -- \\
\hline $05 \mathrm{~A}, \mathrm{~B}$ & 252 & 4.2 & 0.82 & 125 & 25.0 & 7.6 & 503 \\
\hline $06 \mathrm{~A}, \mathrm{~B}$ & 291 & 4.8 & 0.77 & 145 & 25.0 & 7.6 & 506 \\
\hline X02 & 331 & 6.3 & 0.79 & 112 & - & - & - \\
\hline 07B & 336 & 6.4 & 0.77 & 34 & - & - & - \\
\hline 07A & 345 & 6.6 & 0.77 & 140 & 25.0 & 7.8 & 512 \\
\hline 08A,B & 391 & 7.5 & 0.73 & 167 & 25.0 & 7.9 & 512 \\
\hline $\mathrm{X} 01$ & 406 & 7.8 & 0.70 & 119 & -- & -- & -- \\
\hline REW & 435 & 0.0 & 0.00 & & & & \\
\hline MEANS & & 4.5 & 0.74 & & & & \\
\hline TOTAL & 435 & & & 1,436 & & & \\
\hline
\end{tabular}

${ }^{1}$ Specific conductance is questionable since readings of the standard ( 718 microsiemens $/ \mathrm{cm}$ ) drifted from 729 (at the start of the section) to 691 (at the end of the section). 


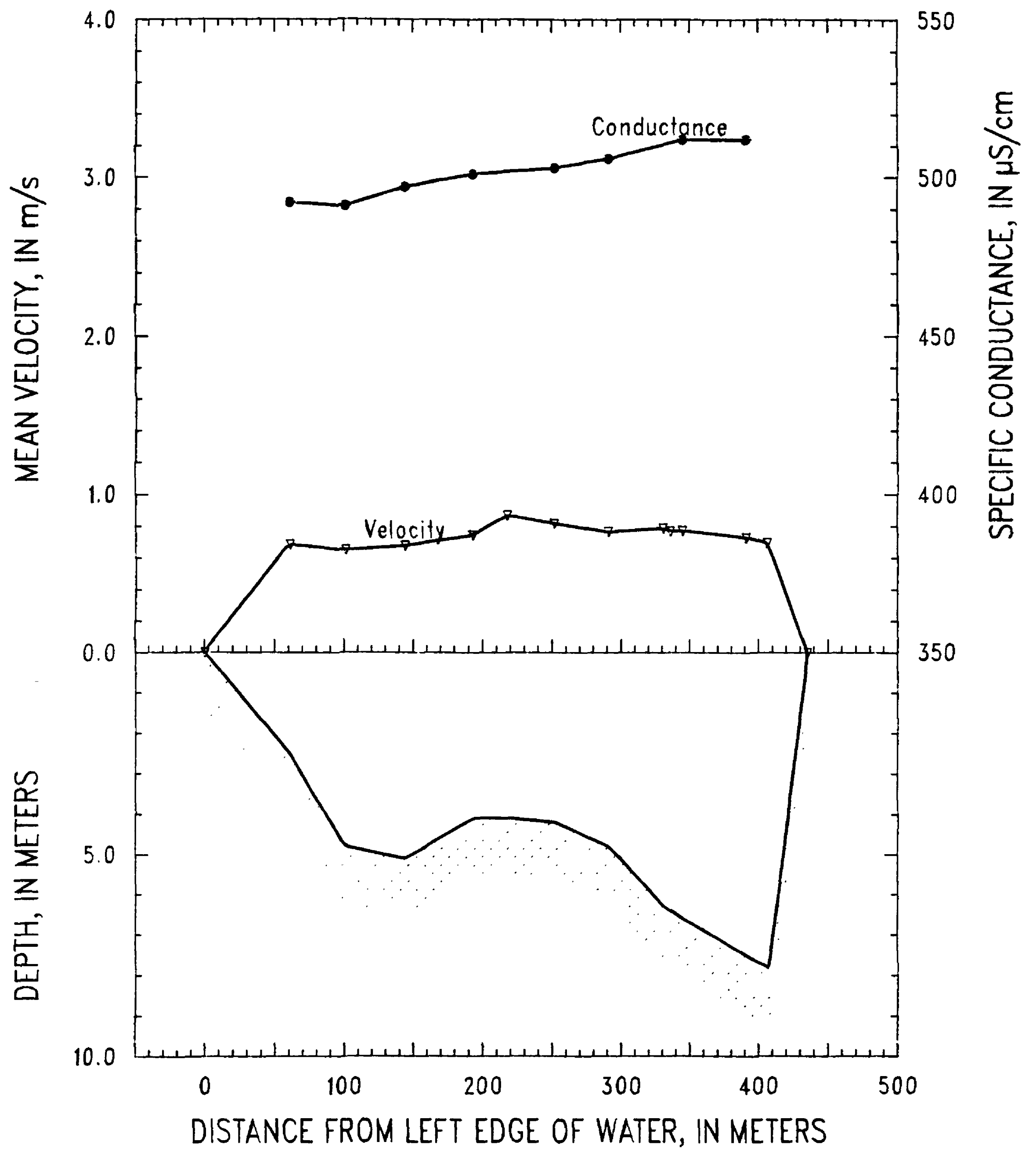

Figure 11. Mississippi River at Trempealeau, Wisconsin, on July 12, 1991. 
SITE: Mississippi River below Lock and Dam 9,Wis.--Mile 639.7

07-15-91

PARTY: Moody, Roth, and Simoneaux

GAGE HEIGHT @ Pool 9 TW: 617.59 ft GAGE HEIGHT @ Pool 10: $610.42 \mathrm{ft}$

RIVER SLOPE: $41 \times 10^{-6}$

SUSP. Bag sampler and 150 -lb weight

PRICE AA CURRENT METER No: W-223906 DATE RATED: 06-91

CURRENT METER EQUATION: $V(\mathrm{~m} / \mathrm{s})=\operatorname{Rev} / \mathrm{s}^{*} 0.670+0.010$

REMARKS:

Unmeasured flow through a shallow side channel was estimated at $30 \mathrm{~m}^{3} / \mathrm{s}$. Sampled nine verticals (equalwidth increment) using a 5/16-inch nozzle and transit rate of $7 \mathrm{~cm} / \mathrm{s}$. Discharge at Dam 9 was reported to be $1,595 \mathrm{~m}^{3} / \mathrm{s}$. Verticals sampled from 9 to 1 .

\begin{tabular}{|c|c|c|c|c|c|c|c|}
\hline Vertical & $\begin{array}{c}\text { Distance } \\
\text { from } \\
\text { LEW } \\
\text { (m) }\end{array}$ & $\begin{array}{l}\text { Depth } \\
\text { (m) }\end{array}$ & $\begin{array}{l}\text { Mean } \\
\text { velocity } \\
(\mathbf{m} / \mathbf{s})\end{array}$ & $\begin{array}{c}\text { Discharge } \\
\left(\mathrm{m}^{3} / \mathrm{s}\right)\end{array}$ & $\begin{array}{c}\text { Temperature } \\
\left({ }^{\circ} \mathrm{C}\right)\end{array}$ & pH & $\begin{array}{c}\text { Specific } \\
\text { conductance } \\
(\mu \mathrm{S} / \mathrm{cm})\end{array}$ \\
\hline LEW & 0 & 0.0 & 0.00 & & & & \\
\hline $01 \mathrm{~B}$ & 49 & 2.8 & 0.44 & 34 & - & -- & - \\
\hline $01 \mathrm{~A}$ & 55 & 3.0 & 0.44 & 16 & 26.3 & 8.2 & 487 \\
\hline $02 \mathrm{~A}, \mathrm{~B}$ & 73 & 3.4 & 0.47 & 58 & 26.2 & 8.2 & 487 \\
\hline 03A,B & 127 & 4.8 & 0.66 & 108 & 26.4 & 8.2 & 488 \\
\hline X05 & 141 & 4.9 & 0.76 & 91 & -- & - & - \\
\hline 04A,B & 176 & 6.0 & 0.72 & 176 & 25.9 & 8.1 & 504 \\
\hline $05 \mathrm{~A}, \mathrm{~B}$ & 222 & 6.2 & 0.83 & 240 & 25.7 & 8.0 & 493 \\
\hline $06 \mathrm{~A}, \mathrm{~B}$ & 270 & 6.7 & 0.83 & 211 & 25.6 & 7.9 & 496 \\
\hline $\mathrm{X03}$ & 298 & 6.4 & 0.88 & 150 & -- & - & - \\
\hline 07A,B & 323 & 6.3 & 0.86 & 181 & 25.1 & 7.9 & 498 \\
\hline$X 01$ & 365 & 5.9 & 0.79 & 121 & - & - & -- \\
\hline 08A,B & 375 & 5.7 & 0.72 & 88 & 25.2 & 7.9 & 500 \\
\hline $\mathrm{X02}$ & 408 & 4.2 & 0.70 & 72 & - & - & -- \\
\hline 09A,B & 424 & 3.8 & 0.55 & 44 & 24.9 & 7.9 & 504 \\
\hline REW & 450 & 0.0 & 0.00 & & & & \\
\hline MEANS & & 4.8 & 0.74 & & & & \\
\hline TOTAL & 450 & & & 1,589 & & & \\
\hline
\end{tabular}




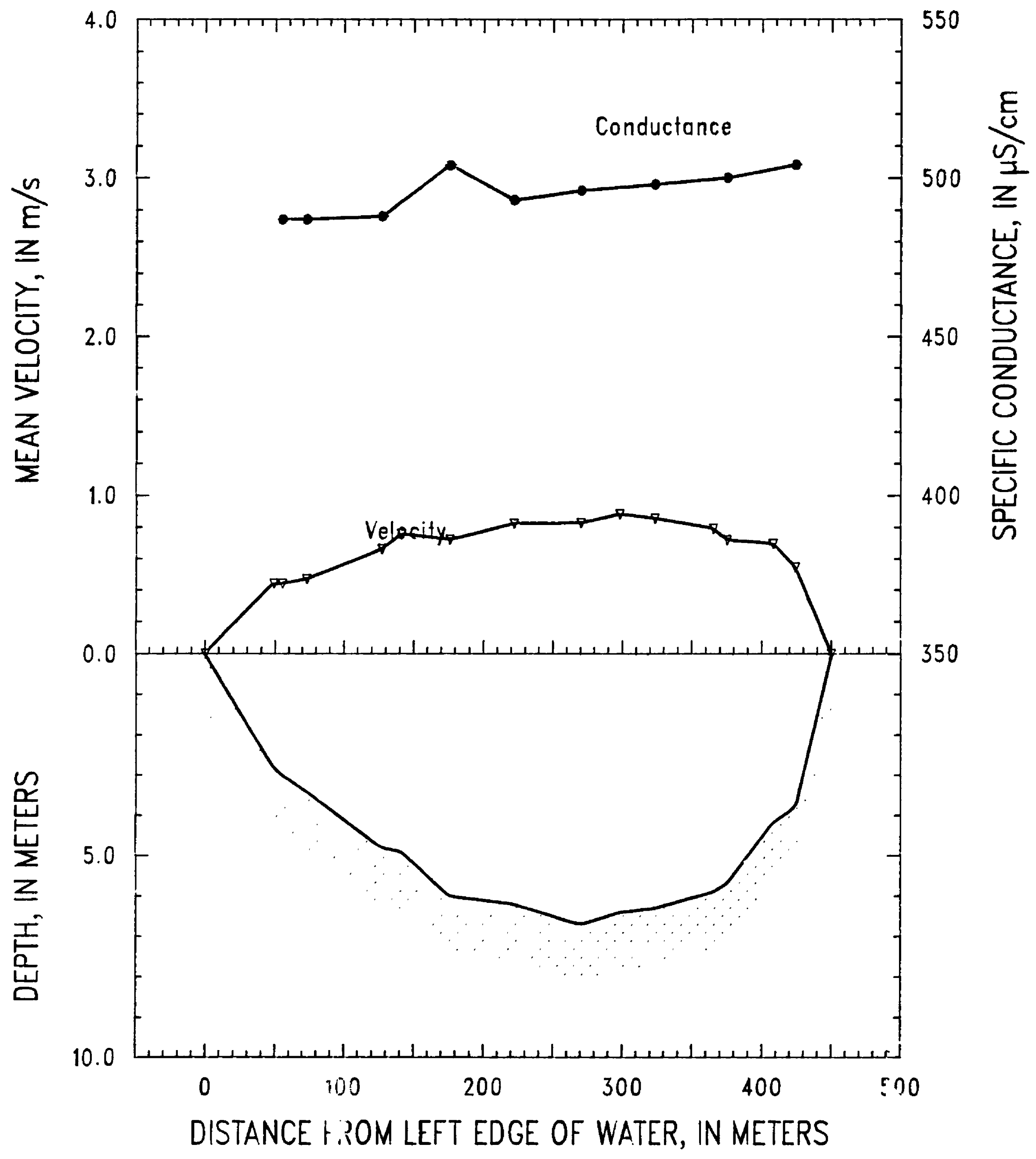

Figure 12. Mississippi River below Lock and Dam 9, Wisconsin, on July 15, 1991. 
SITE: Mississippi River at Clinton, Iowa-Mile 520.3

PARTY: Moody, Roth, and Simoneaux

GAGE HEIGHT @ Dam 13 TW: $576.66 \mathrm{ft}$ GAGE HEIGHT @ Pool 14: $571.93 \mathrm{ft}$

RIVER SLOPE: $30 \times 10^{-6}$

SUSP. Bag sampler and 150-lb weight

PRICE AA CURRENT METER No: W-223906 DATE RATED: 06-91

CURRENT METER EQUATION: $V(\mathrm{~m} / \mathrm{s})=\operatorname{Rev} / \mathrm{s} * 0.670+0.010$

REMARKS:

An additional discharge of $12 \mathrm{~m}^{3} / \mathrm{s}$ adjacent to the left bank (between an island and the bank) which could not be measured from the R/V ACADIANA because of shallow water, was measured from a small boat. Discharge from Dam 13 was about $1,810 \mathrm{~m}^{3} / \mathrm{s}$. Transit rate was $8.0 \mathrm{~cm} / \mathrm{s}$ and a $5 / 16-\mathrm{inch}$ nozzle was used. Order of verticals: 7-10 then, 6-2. Vertical 1 was not sampled because it was in shallow water.

\begin{tabular}{lccccccc}
\hline Vertical & $\begin{array}{c}\text { Distance } \\
\text { from } \\
\text { LEW } \\
(\mathbf{m})\end{array}$ & $\begin{array}{c}\text { Depth } \\
(\mathbf{m})\end{array}$ & $\begin{array}{c}\text { Mean } \\
\text { velocity } \\
(\mathbf{m} / \mathbf{s})\end{array}$ & $\begin{array}{c}\text { Discharge } \\
\left(\mathbf{m}^{3} / \mathbf{s}\right)\end{array}$ & $\begin{array}{c}\text { Temperature } \\
\left({ }^{\circ} \mathbf{C}\right)\end{array}$ & pH & $\begin{array}{c}\text { Specffic } \\
\text { conductance } \\
(\mu \mathbf{S} / \mathbf{c m})\end{array}$ \\
\hline LEW & 0 & 0.0 & 0.00 & & & & \\
02A,B & 87 & 3.0 & 0.33 & 49 & 26.9 & 8.1 & 481 \\
X06 & 101 & 3.4 & 0.36 & 22 & - & - & - \\
03A,B & 124 & 4.3 & 0.35 & 53 & 26.9 & 8.2 & 483 \\
04A,B & 171 & 7.0 & 0.59 & 188 & 26.6 & 8.0 & 484 \\
05A,B & 216 & 7.6 & 0.61 & 169 & 26.7 & 8.0 & 484 \\
X04 & 244 & 7.9 & 0.66 & 118 & -- & - & - \\
06A,B & 261 & 8.3 & 0.70 & 199 & 26.7 & 7.9 & 485 \\
07A,B & 313 & 9.2 & 0.74 & 351 & 26.1 & 7.8 & 483 \\
08A,B & 364 & 8.8 & 0.79 & 248 & 26.1 & 8.1 & 481 \\
X02 & 384 & 9.4 & 0.71 & 148 & - & -- & - \\
09A,B & 408 & 8.9 & 0.68 & 168 & 26.2 & 8.1 & 486 \\
X01 & 440 & 7.0 & 0.66 & 115 & - & - & - \\
10A,B & 458 & 4.7 & 0.22 & 21 & 27.0 & 8.2 & 485 \\
REW & 480 & 0.0 & 0.00 & & & & \\
MEANS & & 6.1 & 0.63 & & & & \\
TOTAL & 480 & 6.1 & 0.63 & 1,850 & & & - \\
\hline
\end{tabular}

64 HYDROLOGIC AND SEDIMENTOLOGIC DATA COLLECTED DURING THREE CRUISES ON THE MISSISSIPPI RIVER AND SOME OF ITS TRIBUTARIES FROM MINNEAPOLIS, MINNESOTA, TO NEW ORLEANS, LOUISIANA, JULY 1991-MAY 1992 


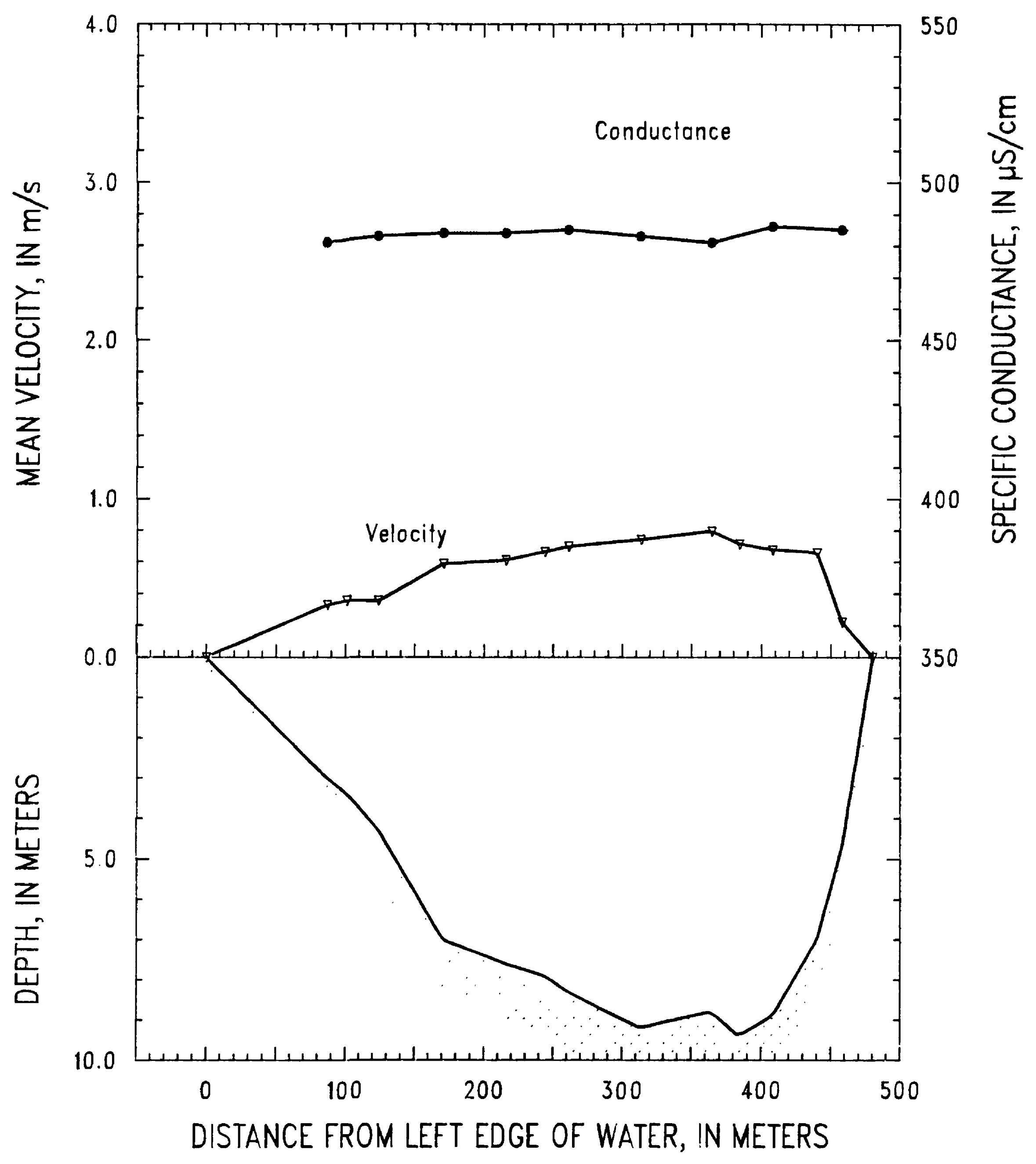

Figure 13. Mississippi River at Clinton, lowa, on July 18, 1991. 
SITE: Mississippi River at Keokuk, Iowa-Mile 363.1

$07-21-91$

PARTY: Moody, Roth, and Simoneaux

GAGE HEIGHT @ Dam 19 TW: $484.06 \mathrm{ft}$ GAGE HEIGHT @ Pool 20: 478.54

RIVER SLOPE: $50 \times 10^{-6}$

SUSP. Bag sampler and 150-lb weight

PRICE AA CURRENT METER No: W-223906 DATE RATED: 06-91

CURRENT METER EQUATION: $V(\mathrm{~m} / \mathrm{s})=\operatorname{Rev} / \mathrm{s}^{*} 0.670+0.010$

REMARKS:

Dam 19 discharge was about $1,915 \mathrm{~m}^{3} / \mathrm{s}$. Discharge increased during the sampling. Nine verticals were sampled from vertical number 10 to 2 . Vertical 1 was not sampled because it was in shallow water. The trathsit rate was $5.0 \mathrm{~cm} / \mathrm{s}$ and the nozzle $w$ as $5 / 16 \mathrm{inch}$. Temperature, $\mathrm{pH}$, and specific conductance are the average of 3-6 individual samples.

\begin{tabular}{|c|c|c|c|c|c|c|c|}
\hline Vertical & $\begin{array}{l}\text { Distance } \\
\text { from } \\
\text { LEW } \\
\text { (m) }\end{array}$ & $\begin{array}{l}\text { Depth } \\
\text { (m) }\end{array}$ & $\begin{array}{c}\text { Mean } \\
\text { veiocity } \\
(\mathrm{m} / \mathrm{s})\end{array}$ & $\begin{array}{c}\text { Discharge } \\
\left(\mathrm{m}^{3} / \mathrm{s}\right)\end{array}$ & $\begin{array}{c}\text { Temperature } \\
\left({ }^{\circ} \mathrm{C}\right)\end{array}$ & pH & $\begin{array}{c}\text { Specific } \\
\text { conductance } \\
(\mu \mathrm{S} / \mathrm{cm})\end{array}$ \\
\hline LEW & 0 & 0.0 & 0.00 & & & & \\
\hline$X 05$ & 69 & 3.2 & 0.38 & 70 & - & - & - \\
\hline $02 \mathrm{~A}, \mathrm{~B}$ & 114 & 3.5 & 0.51 & 115 & 28.5 & 8.1 & 480 \\
\hline 03A,B & 198 & 4.1 & 0.53 & 113 & 29.6 & 8.0 & 480 \\
\hline X04 & 219 & 3.9 & 0.53 & 81 & - & - & - \\
\hline $04 \mathrm{~A}, \mathrm{~B}$ & 277 & 3.9 & 0.72 & 187 & 29.6 & 8.1 & 480 \\
\hline 05A,B & 352 & 4.3 & 0.84 & 181 & 29.3 & 8.0 & 481 \\
\hline $\mathrm{X} 03$ & 378 & 4.2 & 0.76 & 120 & - & - & - \\
\hline 06A,B & 427 & 4.3 & 0.80 & 221 & 28.8 & 8.0 & 481 \\
\hline 07A,B & 507 & 5.1 & 0.91 & 247 & 29.1 & 7.9 & 481 \\
\hline $\mathrm{X} 02$ & 534 & 4.5 & 0.84 & 138 & - & - & - \\
\hline OBA,B & 580 & 4.7 & 0.95 & 273 & 29.3 & 8.2 & 484 \\
\hline $09 A, B$ & 656 & 3.9 & 0.79 & 178 & 28.4 & 8.4 & 485 \\
\hline$X_{01}$ & 696 & 3.9 & 0.63 & 84 & - & - & -- \\
\hline $10 \mathrm{~A}, \mathrm{~B}$ & 724 & 2.7 & 0.37 & 39 & 28.4 & 8.4 & 483 \\
\hline REW & 773 & 0.0 & 0.00 & & & & \\
\hline MEANS & & 3.7 & 0.71 & & & & \\
\hline TOTAL & 773 & 3.7 & 0.71 & 2,047 & & & \\
\hline
\end{tabular}




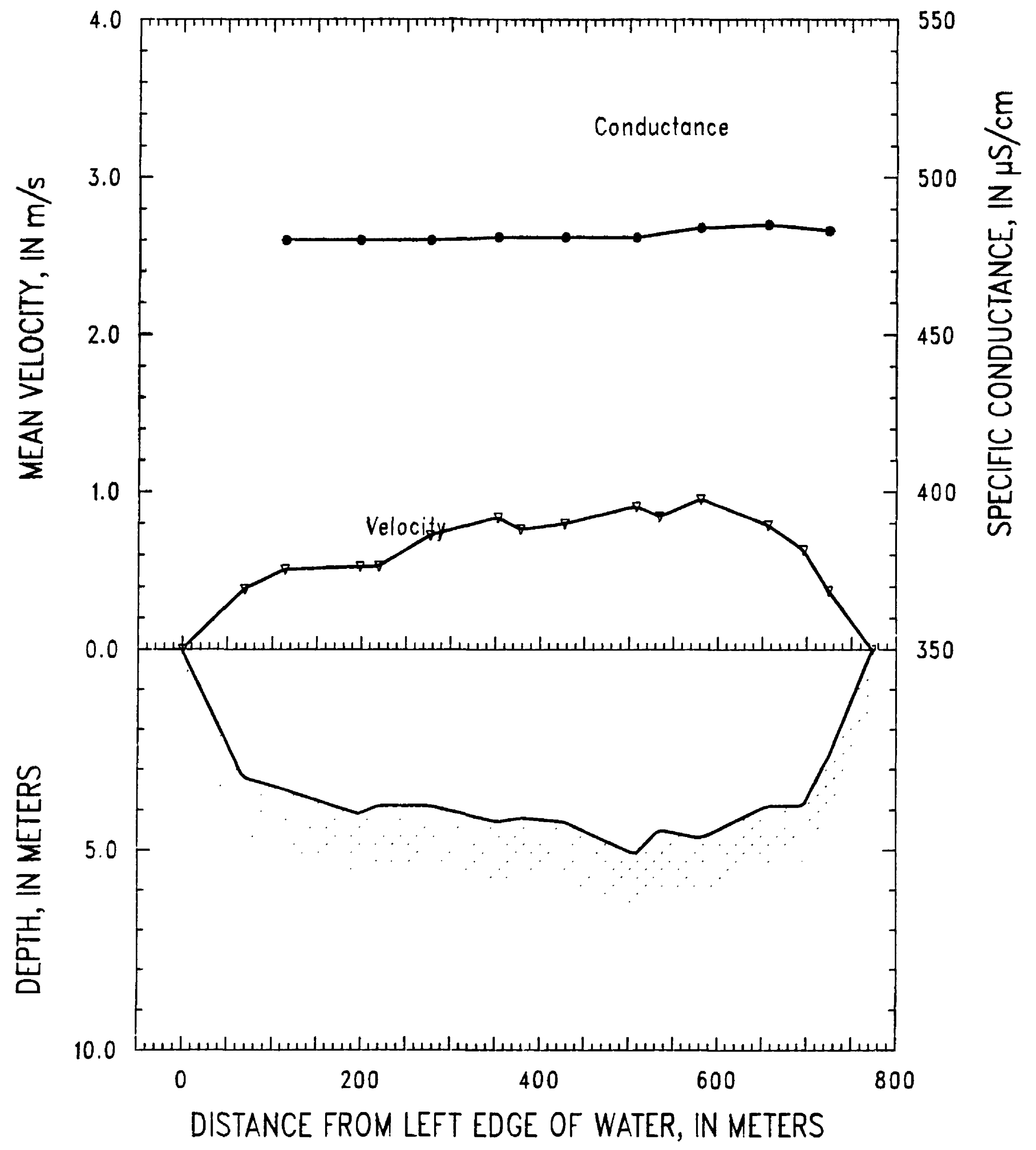

Figure 14. Mississippi River at Keokuk, lowa, on July 21, 1991. 
SITE: Mississippi River near Winfield, Mo.-Mile 239.2

$07-24-91$

PARTY: Moody, Roth, and Simoneaux

GAGE HEIGHT @ Dam 25 TW: $425.78 \mathrm{ft}$ GAGE HEIGHT @ Pool 26: $418.40 \mathrm{ft}$

RIVER SLOPE: $35 \times 10^{-6}$

SUSP. Bag sampler and 150 -lb weight

PRICE AA CURRENT METER No: W-223906 DATE RATED: 06-91

CURRENT METER EQUATION: $\mathrm{V}(\mathrm{m} / \mathrm{s})=\operatorname{Rev} / \mathrm{s}^{*} 0.670+0.010$

REMARKS:

Transit rate was $7.5 \mathrm{~cm} / \mathrm{s}$ and nozzle was $1 / 4 \mathrm{inch}$. Temperature, $\mathrm{pH}$, and specific conductance are the average of three separate samples.

\begin{tabular}{|c|c|c|c|c|c|c|c|}
\hline Vertical & $\begin{array}{l}\text { Distance } \\
\text { from } \\
\text { LEW } \\
\text { (m) }\end{array}$ & $\begin{array}{l}\text { Depth } \\
\text { (m) }\end{array}$ & $\begin{array}{c}\text { Mean } \\
\text { velocity } \\
(\mathbf{m} / \mathbf{s})\end{array}$ & $\begin{array}{c}\text { Discharge } \\
\left(\mathrm{m}^{3} / \mathrm{s}\right)\end{array}$ & $\begin{array}{c}\text { Temperature } \\
\left({ }^{\circ} \mathrm{C}\right)\end{array}$ & pH & $\begin{array}{c}\text { Specific } \\
\text { conductance } \\
(\mu \mathrm{S} / \mathrm{cm})\end{array}$ \\
\hline LEW & 0 & 0.0 & 0.00 & & & & \\
\hline $01 \mathrm{~A}, \mathrm{~B}$ & 29 & 5.9 & 0.51 & 105 & 27.7 & 7.9 & 478 \\
\hline$X_{01}$ & 70 & 8.5 & 0.95 & 234 & - & - & - \\
\hline $02 \mathrm{~A}, \mathrm{~B}$ & 87 & 8.2 & 1.00 & 243 & 27.4 & 8.0 & 474 \\
\hline $\mathrm{X} 02$ & 129 & 7.7 & 1.03 & 247 & - & - & - \\
\hline $03 A, B$ & 149 & 8.1 & 0.96 & 244 & 27.7 & 8.0 & 473 \\
\hline $\mathrm{X} 03$ & 192 & 8.1 & 0.89 & 187 & - & -- & - \\
\hline 04A,B & 201 & 8.0 & 0.85 & 228 & 27.5 & 8.1 & 473 \\
\hline 05A,B & 259 & 6.8 & 0.79 & 251 & 27.5 & 8.1 & 468 \\
\hline X04 & 295 & 6.0 & 0.81 & 147 & - & - & - \\
\hline $06 \mathrm{~A}, \mathrm{~B}$ & 319 & 7.4 & 0.75 & 209 & 27.7 & 8.0 & 467 \\
\hline $07 \mathrm{~A}, \mathrm{~B}$ & 370 & 5.7 & 0.68 & 133 & 27.6 & 8.1 & 462 \\
\hline X05 & 388 & 5.4 & 0.85 & 133 & - & -- & -- \\
\hline $08 \mathrm{~A}, \mathrm{~B}$ & 428 & 5.1 & 0.63 & 134 & 27.2 & 8.1 & 462 \\
\hline$X 06$ & 471 & 4.0 & 0.56 & 64 & -- & - & - \\
\hline $09 \mathrm{~A}, \mathrm{~B}$ & 486 & 4.2 & 0.64 & 86 & 27.5 & 8.1 & 468 \\
\hline $10 A, B$ & 535 & 3.9 & 0.51 & 83 & 27.4 & 8.1 & 469 \\
\hline REW & 570 & 0.0 & 0.00 & & & & \\
\hline MEANS & & 6.0 & 0.80 & & & & \\
\hline TOTAL & 570 & & & 2,729 & & & \\
\hline
\end{tabular}




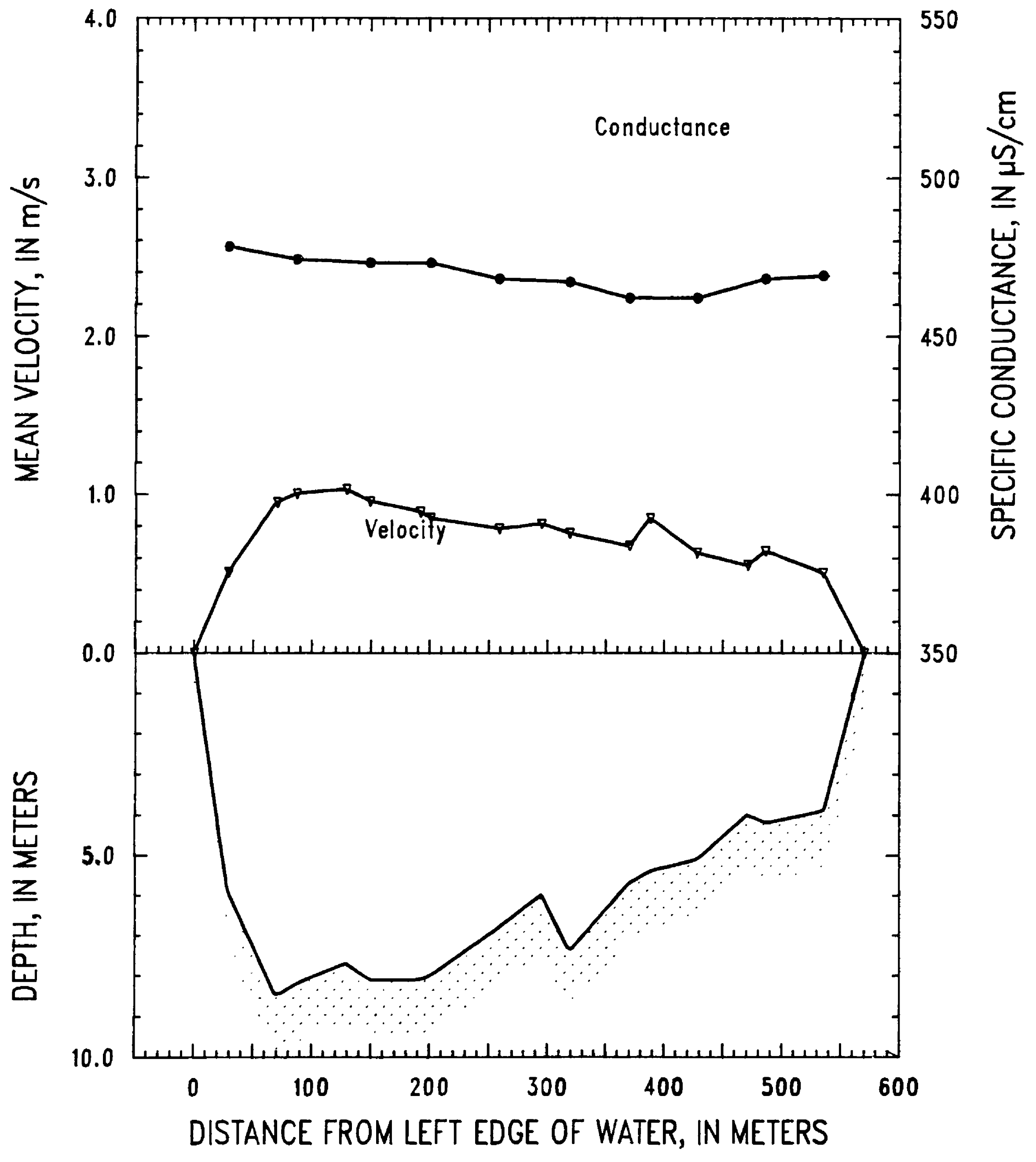

Figure 15. Mississippi River near Winfield, Missouri, on July 24, 1991. 
SITE: Illinois River at Hardin, Ill.-Mile 21.8

07-25-91

PARTY: Moody and Simoneaux

GAGE HEIGHT @ Meredosia: 439.4 ft GAGE HEIGHT @ Grafton: $419.7 \mathrm{ft}$

RIVER SLOPE: $52 \times 10^{-6}$

SUSP. 30-lb weight

PRICE AA CURRENT METER No: 90JM1 DATE RATED: 06-91

CURRENT METER EQUATION: $\mathrm{V}(\mathrm{m} / \mathrm{s})=\operatorname{Rev} / \mathrm{s}^{*} 0.667+0.006$

REMARKS:

Velocities were measured from the small boat at 0.6 depth. ACADIANA was anchored near midriver. A depth-integrated sample was collected without a nozzle. The sample for trace metal analysis was collected by pumping $104 \mathrm{~L}$ after the pump sample $(854 \mathrm{~L})$ was collected. Temperature and specific conductance were measured with LabComp instrument, instead of the Beckman and Amber Science meters.

\begin{tabular}{lccccccc}
\hline Vertical & $\begin{array}{c}\text { Distance } \\
\text { from } \\
\text { LEW } \\
(\mathbf{m})\end{array}$ & $\begin{array}{c}\text { Depth } \\
(\mathbf{m})\end{array}$ & $\begin{array}{c}\text { Mean } \\
\text { velocity } \\
(\mathbf{m} / \mathbf{s})\end{array}$ & $\begin{array}{c}\text { Discharge } \\
\left(\mathbf{m}^{3} / \mathbf{s}\right)\end{array}$ & $\begin{array}{c}\text { Temperature } \\
\left({ }^{\circ} \mathbf{C}\right)\end{array}$ & pH & $\begin{array}{c}\text { Spocific } \\
\text { conductance } \\
(\mu \mathbf{S} / \mathbf{c m})\end{array}$ \\
\hline LEW & 0 & 0.0 & 0.00 & & & & \\
01 & 23 & 1.0 & 0.12 & 3 & 28.4 & - & 712 \\
02 & 49 & 3.3 & 0.14 & 11 & 28.5 & - & 714 \\
03 & 71 & 4.2 & 0.13 & 14 & 28.7 & - & 716 \\
04 & 100 & 4.1 & 0.21 & 23 & 28.9 & - & 716 \\
05 & 123 & 4.0 & 0.27 & 22 & 29.0 & - & 713 \\
06 & 141 & 4.5 & 0.29 & 27 & 28.7 & -- & 716 \\
07 & 164 & 5.7 & 0.29 & 40 & 28.8 & - & 717 \\
08 & 189 & 6.0 & 0.22 & 29 & 28.7 & - & 716 \\
09 & 207 & 6.0 & 0.27 & 35 & 28.9 & - & 718 \\
10 & 232 & 5.0 & 0.22 & 20 & 29.0 & - & 718 \\
11 & 243 & 3.9 & 0.30 & 17 & 29.0 & - & 716 \\
12 & 261 & 2.3 & 0.44 & 22 & 29.0 & - & 718 \\
REW & 286 & 0.0 & 0.00 & & & & \\
MEANS & & 3.8 & 0.24 & & & & \\
TOTAL & 286 & & & 262 & & & \\
\hline & & & & & & & \\
\hline
\end{tabular}




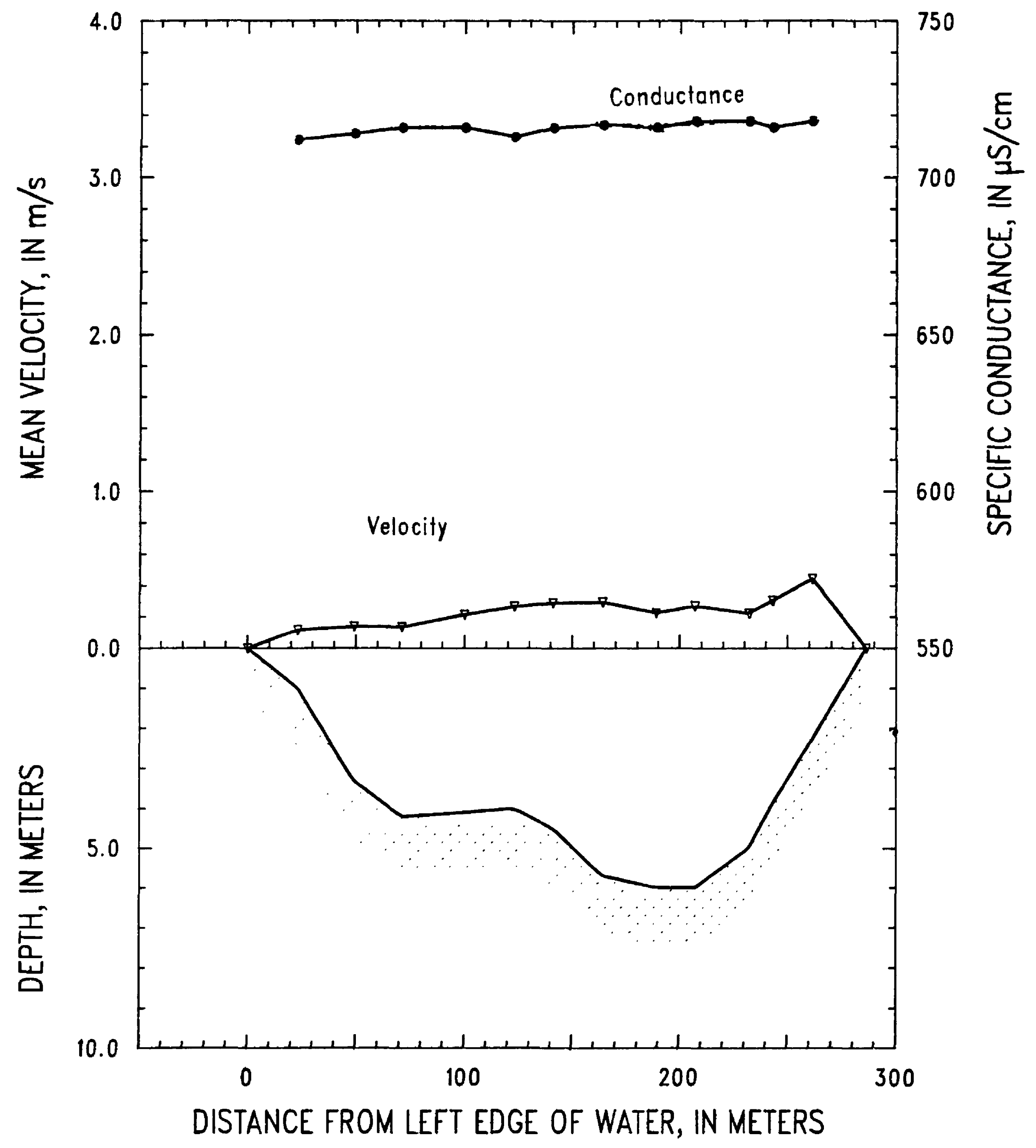

Figure 16. Illinois River at Hardin, Illinois, on July 25, 1991. 
SITE: Missouri River at St. Charles, Mo.-Mile 24.8

07-27-91

PARTY: Moody, Antweiler, and Simoneaux

GAGE HEIGHT @ Hermann: 487.46 ft GAGE HEIGHT @ St. Charles: $431.7 \mathrm{ft}$

RIVER SLOPE: $151 \times 10^{-6}$

SUSP. Bag sampler and 150-lb weight

PRICE AA CURRENT METER No: 90JM1 DATE RATED: 06-91

CURRENT METER EQUATION: $\mathrm{V}(\mathrm{m} / \mathrm{s})=\operatorname{Rev} / \mathrm{s}^{*} 0.667+0.006$

REMARKS:

Transit rate was $5 \mathrm{~cm} / \mathrm{s}$ and the nozzle was $1 / 4 \mathrm{inch}$. Towboat reported sunk at about river mile 90 on July 26, 1991. Temperature, $\mathrm{pH}$, and specific conductance are the average of three separate samp'es.

\begin{tabular}{|c|c|c|c|c|c|c|c|}
\hline Vertical & $\begin{array}{l}\text { Distance } \\
\text { from } \\
\text { LEW } \\
\text { (m) }\end{array}$ & $\begin{array}{l}\text { Depth } \\
\text { (m) }\end{array}$ & $\begin{array}{l}\text { Mean } \\
\text { velocity } \\
(\mathrm{m} / \mathrm{s})\end{array}$ & $\begin{array}{c}\text { Discharge } \\
\left(\mathrm{m}^{3} / \mathrm{s}\right)\end{array}$ & $\begin{array}{c}\text { Temperature } \\
\left({ }^{\circ} \mathrm{C}\right)\end{array}$ & pH & $\begin{array}{c}\text { Specific } \\
\text { conductance } \\
(\mu \mathrm{S} / \mathrm{cm})\end{array}$ \\
\hline LEW & 0 & 0.0 & 0.00 & & & & \\
\hline 01A,B & 33 & 5.4 & 1.01 & 111 & 26.9 & 7.4 & 671 \\
\hline $02 \mathrm{~A}, \mathrm{~B}$ & 41 & 5.4 & 0.99 & 37 & 26.5 & 7.6 & 668 \\
\hline $\mathrm{X} 01$ & 47 & 4.7 & 1.03 & 34 & -- & -- & -- \\
\hline 03A,B & 55 & 4.4 & 1.06 & 56 & 27.1 & 7.5 & 665 \\
\hline $04 \mathrm{~A}, \mathrm{~B}$ & 71 & 4.1 & 1.09 & 72 & 27.2 & 7.6 & 681 \\
\hline 05A,B & 87 & 3.8 & 1.06 & 70 & 27.1 & 7.6 & 681 \\
\hline 06A,B & 106 & 4.1 & 0.96 & 63 & 27.5 & 7.8 & 683 \\
\hline 07A,B & 119 & 3.6 & 1.13 & 69 & 27.2 & 7.6 & 684 \\
\hline 08A,B & 140 & 3.7 & 1.03 & 66 & 26.8 & 7.9 & 681 \\
\hline 09A,B & 154 & 3.7 & 1.12 & 67 & 27.1 & 7.7 & 681 \\
\hline $10 \mathrm{~A}, \mathrm{~B}$ & 172 & 3.7 & 1.01 & 67 & 27.3 & 7.7 & 680 \\
\hline $11 \mathrm{~A}, \mathrm{~B}$ & 190 & 3.8 & 1.10 & 78 & 27.4 & 7.8 & 681 \\
\hline $12 A, B$ & 209 & 3.9 & 1.04 & 71 & 27.5 & 7.7 & 682 \\
\hline $13 \mathrm{~A}, \mathrm{~B}$ & 225 & 3.9 & 1.16 & 57 & 27.4 & 7.8 & 680 \\
\hline $\mathrm{X} 05$ & 234 & 4.0 & 0.99 & 30 & -- & - & -- \\
\hline $14 A, B$ & 240 & 4.4 & 1.05 & 58 & 27.3 & 8.0 & 681 \\
\hline $15 \mathrm{~A}, \mathrm{~B}$ & 259 & 4.1 & 1.15 & 92 & 27.5 & 7.8 & 684 \\
\hline REW & 279 & 0.0 & 0.00 & & & & \\
\hline MEANS & & 3.7 & 1.06 & & & & \\
\hline TOTAL & 279 & & & 1,096 & & & \\
\hline
\end{tabular}




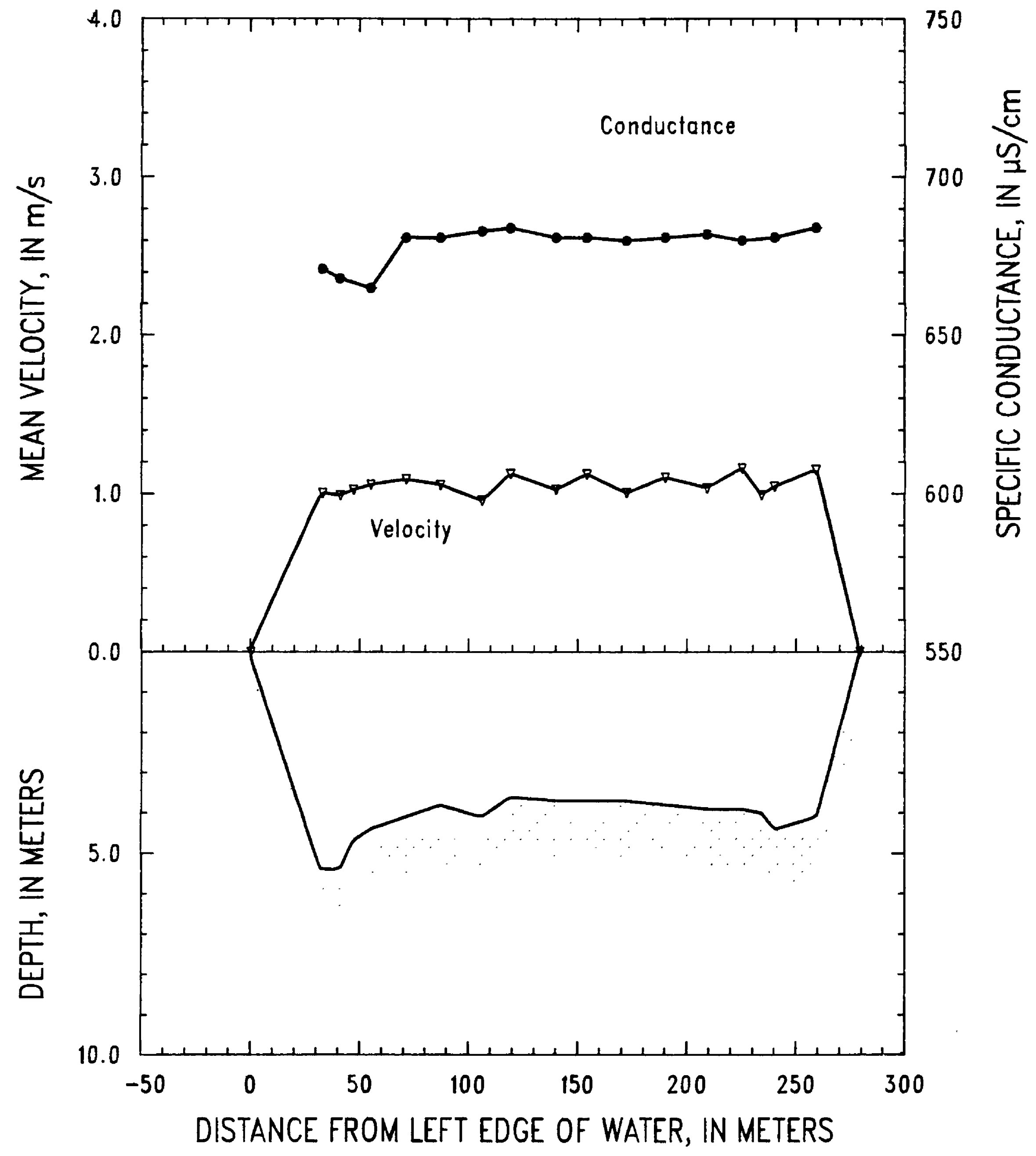

Figure 17. Missouri River at St. Charles, Missouri, on July 27, 1991. 
SITE: Mississippi River at Thebes, Ill.-Mile 44.0

07-29-91

PARTY: Moody, Ellis, and Simoneaux

GAGE HEIGHT @ Chester: $351.7 \mathrm{ft}$ GAGE HEIGHT @ Cape Girardeau: $320.4 \mathrm{ft}$

RIVER SLOPE: $102 \times 10^{-6}$

SUSP. Bag sampler and 150-lb weight

PRICE AA CURRENT METER No: W-223906 DATE RATED: 06-91

CURRENT METER EQUATION: $\mathrm{V}(\mathrm{m} / \mathrm{s})=\operatorname{Rev} / \mathrm{s}^{*} 0.670+0.010$

REMARKS:

Transit rate was $10 \mathrm{~cm} / \mathrm{s}$ and the nozzle was $1 / 4 \mathrm{inch}$. Order of verticals was $1-7$, then 15-8. Temperature, $\mathrm{pH}$, and specific conductance are the average of three separate samples.

\begin{tabular}{|c|c|c|c|c|c|c|c|}
\hline Vertical & $\begin{array}{l}\text { Distance } \\
\text { from } \\
\text { LEW } \\
\text { (m) }\end{array}$ & $\begin{array}{c}\text { Depth } \\
\text { (m) }\end{array}$ & $\begin{array}{l}\text { Mean } \\
\text { velocity } \\
(\mathbf{m} / \mathbf{s})\end{array}$ & $\begin{array}{c}\text { Discharge } \\
\left(\mathrm{m}^{3} / \mathrm{s}\right)\end{array}$ & $\begin{array}{c}\text { Temperature } \\
\left({ }^{\circ} \mathrm{C}\right)\end{array}$ & pH & $\begin{array}{c}\text { Spocific } \\
\text { conductance } \\
(\mu \mathrm{S} / \mathrm{cm})\end{array}$ \\
\hline LEW & 0 & 0.0 & 0.00 & & & & \\
\hline 01A,B & 39 & 6.6 & 0.40 & 95 & 27.5 & 8.0 & 538 \\
\hline 02A,B & 72 & 8.2 & 0.73 & 158 & 27.3 & 8.1 & 539 \\
\hline $\mathrm{X} 05$ & 92 & 7.8 & 1.02 & 139 & -- & -- & -- \\
\hline 03A,B & 107 & 8.1 & 1.12 & 291 & 27.0 & 8.0 & 539 \\
\hline 04A,B & 156 & 8.4 & 1.45 & 495 & 27.4 & 8.2 & 539 \\
\hline 05A,B & 188 & 8.4 & 1.49 & 262 & 27.3 & 8.4 & 544 \\
\hline$X 06$ & 198 & 8.5 & 1.47 & 188 & - & - & -- \\
\hline 06A,B & 218 & 8.6 & 1.43 & 339 & 27.2 & 8.2 & 544 \\
\hline 07A,B & 253 & 9.1 & 1.28 & 443 & 27.6 & 8.1 & 544 \\
\hline 08A,B & 294 & 8.6 & 1.28 & 419 & 27.6 & 8.2 & 544 \\
\hline 09A,B & 329 & 8.3 & 1.20 & 337 & 27.6 & 8.2 & 543 \\
\hline $10 \mathrm{~A}, \mathrm{~B}$ & 362 & 7.3 & 1.12 & 278 & 27.5 & 8.2 & 544 \\
\hline $11 \mathrm{~A}, \mathrm{~B}$ & 397 & 6.7 & 1.16 & 291 & 27.7 & 8.2 & 545 \\
\hline $12 \mathrm{~A}, \mathrm{~B}$ & 437 & 6.8 & 1.08 & 294 & 27.7 & 8.2 & 546 \\
\hline $13 \mathrm{~A}, \mathrm{~B}$ & 477 & 5.3 & 0.97 & 196 & 28.3 & 8.2 & 546 \\
\hline $14 \mathrm{~A}, \mathrm{~B}$ & 513 & 4.8 & 0.72 & 76 & 28.3 & 8.2 & 546 \\
\hline$X 01$ & 521 & 4.7 & 0.71 & 52 & -- & -- & - \\
\hline $15 A, B$ & 544 & 3.9 & 0.34 & 42 & 28.7 & 8.1 & 545 \\
\hline REW & 584 & 0.0 & 0.00 & & & & \\
\hline MEANS & & 6.8 & 1.11 & & & & \\
\hline TOTAL & 584 & & & 4,394 & & & \\
\hline
\end{tabular}




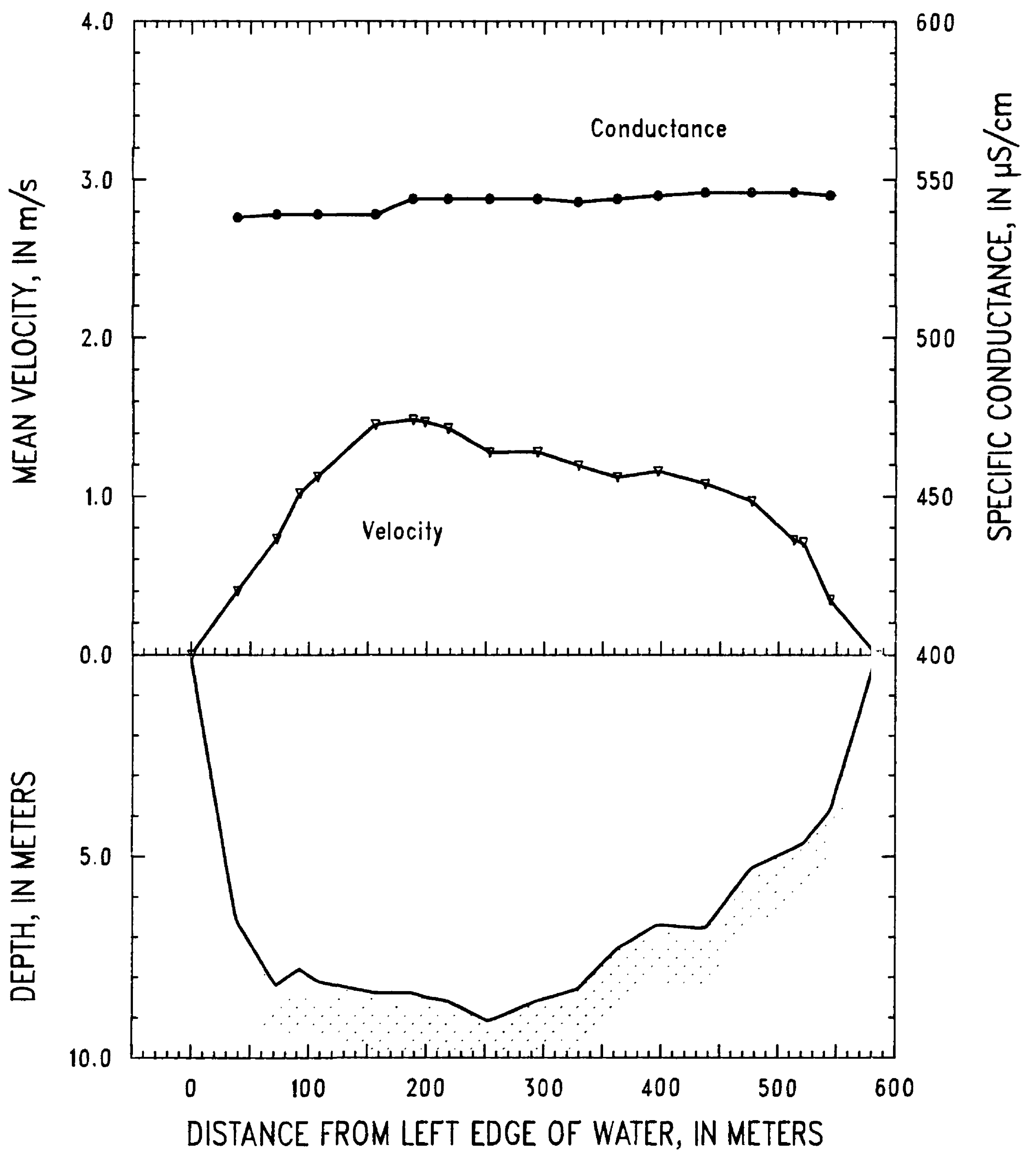

Figure 18. Mississippi River at Thebes, Illinois, on July 29, 1991. 
SITE: Ohio River at Olmsted, Ill.-Mile 965.5

07-30-91

PARTY: Moody, Ellis, and LeBoeuf

GAGE HEIGHT @ Dam 53: $287.7 \mathrm{ft}$ GAGE HEIGHT @ Cairo: $286.3 \mathrm{ft}$

RIVER SLOPE: $14 \times 10^{-6}$

SUSP. Bag sampler and $150-1 \mathrm{~b}$ weight

PRICE AA CURRENT METER No: W-223906 DATE RATED: 06-91

CURRENT METER EQUATION: $\mathrm{V}(\mathrm{m} / \mathrm{s})=\operatorname{Rev} / \mathrm{s}^{*} 0.670+0.010$

REMARKS:

Water velocities were too slow $(\sim 30 \mathrm{~cm} / \mathrm{sec})$ to collect depth-integrated samples. Order of verticals was 11 , then 1-15. Anchored at each vertical to measure velocity and collect discharge-weighted pump sample.

\begin{tabular}{|c|c|c|c|c|c|c|c|}
\hline Vertical & $\begin{array}{l}\text { Distance } \\
\text { from } \\
\text { LEW } \\
\text { (m) }\end{array}$ & $\begin{array}{l}\text { Depth } \\
\text { (m) }\end{array}$ & $\begin{array}{l}\text { Mean } \\
\text { velocity } \\
(\mathbf{m} / \mathbf{s})\end{array}$ & $\begin{array}{c}\text { Discharge } \\
\left(\mathrm{m}^{3} / \mathrm{s}\right)\end{array}$ & $\begin{array}{c}\text { Temperature } \\
\left({ }^{\circ} \mathrm{C}\right)\end{array}$ & pH & $\begin{array}{c}\text { Speciflc } \\
\text { conductance } \\
(\mu \mathrm{S} / \mathrm{cm})\end{array}$ \\
\hline LEW & 0 & 0.0 & & & & & \\
\hline 01 & 49 & 5.7 & 0.21 & 42 & 28.6 & 8.0 & 210 \\
\hline$X 06$ & 69 & 6.3 & 0.24 & 45 & -- & -- & -- \\
\hline 02 & 107 & 7.4 & 0.28 & 87 & 28.8 & 8.2 & 212 \\
\hline 03 & 152 & 8.5 & 0.39 & 100 & 28.7 & 8.1 & 221 \\
\hline X05 & 167 & 8.7 & 0.34 & 72 & -- & -- & -- \\
\hline 04 & 200 & 8.5 & 0.39 & 134 & 28.9 & 8.2 & 222 \\
\hline 05 & 249 & 8.0 & 0.45 & 171 & 29.0 & 8.1 & 228 \\
\hline 06 & 295 & 8.7 & 0.40 & 126 & 28.9 & 8.1 & 225 \\
\hline X04 & 321 & 7.9 & 0.46 & 85 & -- & - & -- \\
\hline 07 & 342 & 7.6 & 0.44 & 115 & 29.1 & 8.1 & 226 \\
\hline 08 & 389 & 8.9 & 0.42 & 178 & 29.0 & 8.1 & 219 \\
\hline 09 & 438 & 8.2 & 0.42 & 97 & 29.0 & 8.1 & 224 \\
\hline $\mathrm{X} 01$ & 445 & 7.7 & 0.45 & 59 & - & -- & -- \\
\hline 10 & 472 & 8.0 & 0.44 & 180 & 29.1 & 8.1 & 229 \\
\hline 11 & 547 & 10.7 & 0.39 & 204 & 28.8 & 8.1 & 267 \\
\hline 12 & 571 & 10.3 & 0.42 & 129 & 29.1 & 8.1 & 260 \\
\hline$X 03$ & 606 & 9.6 & 0.36 & 84 & -- & - & -- \\
\hline 13 & 620 & 9.7 & 0.42 & 160 & 29.1 & 8.1 & 264 \\
\hline 14 & 684 & 10.4 & 0.38 & 216 & 29.1 & 8.1 & 277 \\
\hline X02 & 730 & 9.9 & 0.28 & 80 & -- & - & -- \\
\hline 15 & 742 & 10.4 & 0.19 & 49 & 29.1 & 8.1 & 284 \\
\hline REW & 780 & 0.0 & 0.00 & & & & \\
\hline MEANS & & 8.2 & 0.38 & & & & \\
\hline TOTAL & 780 & & & 2,410 & & & \\
\hline
\end{tabular}




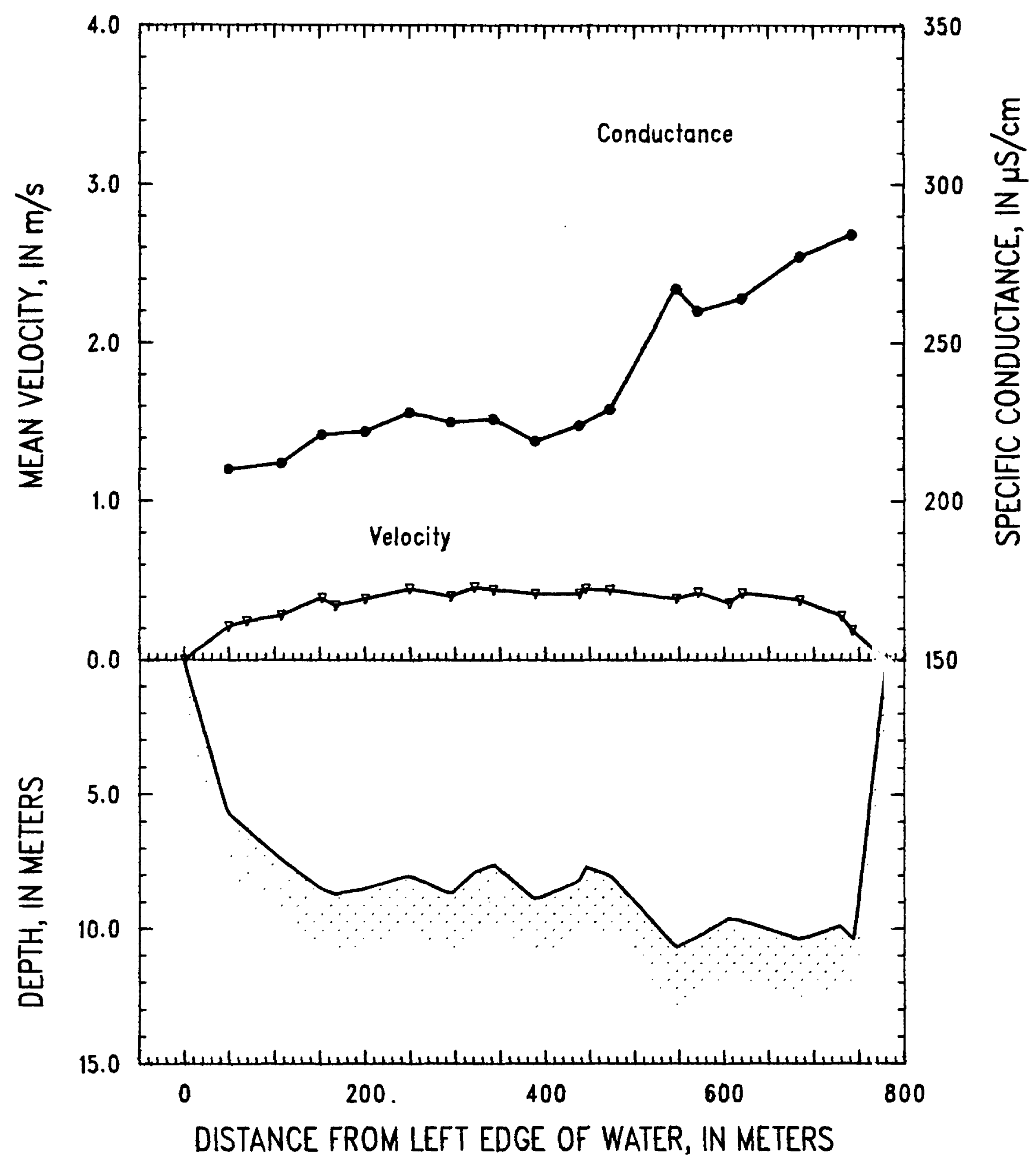

Figure 19. Ohio River at Olmsted, Illinois, on July 30, 1991. 
SITE: Mississippi River below Vicksburg, Miss.-Mile 433.4

08-03-91

PARTY: Moody, Ellis, and LeBoeuf

GAGE HEIGHT @ Greenville: $91.6 \mathrm{ft}$ GAGE HEIGHT @ Natchez: $32.9 \mathrm{ft}$

RIVER SLOPE: $66 \times 10^{-6}$

SUSP. Bag sampler and 150 -lb weight

PRICE AA CURRENT METER No: W-223906 DATE RATED: 06-91

CURRENT METER EQUATION: $V(\mathrm{~m} / \mathrm{s})=\operatorname{Rev} / \mathrm{s}^{*} 0.670+0.010$

REMARKS:

Twenty equally spaced verticals were planned, but only 17 were sampled owing to shallow water along the right edge of water. Transit rate was $7.0 \mathrm{~cm} / \mathrm{s}$ and the nozzle was $3 / 16 \mathrm{inch}$. Temperature, $\mathrm{pH}$, and specific conductance are the average of three separate samples.

\begin{tabular}{|c|c|c|c|c|c|c|c|}
\hline Vertical & $\begin{array}{l}\text { Distance } \\
\text { from } \\
\text { LEW } \\
\text { (m) }\end{array}$ & $\begin{array}{l}\text { Depth } \\
\text { (m) }\end{array}$ & $\begin{array}{l}\text { Mean } \\
\text { velocity } \\
(\mathbf{m} / \mathbf{s})\end{array}$ & $\begin{array}{c}\text { Discharge } \\
\left(\mathrm{m}^{3} / \mathrm{s}\right)\end{array}$ & $\begin{array}{c}\text { Temperature } \\
\left({ }^{\circ} \mathrm{C}\right)\end{array}$ & pH & $\begin{array}{c}\text { Speclfic } \\
\text { conductance } \\
(\mu \mathrm{S} / \mathrm{cm})\end{array}$ \\
\hline LEW & 0 & 0.0 & 0.00 & & & & \\
\hline $01 \mathrm{~A}, \mathrm{~B}$ & 54 & 6.0 & 0.47 & 102 & 28.9 & 8.0 & 392 \\
\hline X01 & 72 & 7.0 & 0.69 & 156 & - & -- & - \\
\hline $02 \mathrm{~A}, \mathrm{~B}$ & 118 & 14.6 & 1.01 & 621 & 28.9 & 8.0 & 394 \\
\hline X02 & 156 & 13.9 & 1.12 & 335 & - & -- & - \\
\hline X03 & 161 & 13.8 & 1.14 & 87 & -- & -- & -- \\
\hline 03A,B & 167 & 13.7 & 1.12 & 415 & 29.0 & 8.0 & 404 \\
\hline $04 \mathrm{~A}, \mathrm{~B}$ & 215 & 12.8 & 1.14 & 776 & 29.0 & 8.0 & 406 \\
\hline 05A,B & 273 & 12.4 & 1.15 & 832 & 29.2 & 8.1 & 413 \\
\hline 06A,B & 332 & 11.8 & 1.07 & 745 & 29.2 & 8.1 & 420 \\
\hline 07A,B & 391 & 11.1 & 1.06 & 589 & 29.2 & 8.1 & 423 \\
\hline X04 & 432 & 11.4 & 0.97 & 342 & -. & - & -- \\
\hline 08A,B & 453 & 10.8 & 1.03 & 406 & 29.2 & 8.1 & 426 \\
\hline $09 \mathrm{~A}, \mathrm{~B}$ & 505 & 10.3 & 1.01 & 570 & 29.4 & 8.1 & 430 \\
\hline $10 A, B$ & 563 & 9.8 & 1.04 & 618 & 29.2 & 8.1 & 435 \\
\hline $11 \mathrm{~A}, \mathrm{~B}$ & 626 & 10.3 & 0.89 & 518 & 29.4 & 8.1 & 437 \\
\hline $12 \mathrm{~A}, \mathrm{~B}$ & 676 & 9.7 & 0.87 & 361 & 29.3 & 8.1 & 438 \\
\hline X05 & 712 & 8.9 & 0.84 & 216 & -- & -- & -- \\
\hline $13 A, B$ & 734 & 8.6 & 0.78 & 243 & 29.3 & 8.1 & 440 \\
\hline $14 \mathrm{~A}, \mathrm{~B}$ & 784 & 7.2 & 0.71 & 291 & 29.5 & 8.1 & 440 \\
\hline $15 \mathrm{~A}, \mathrm{~B}$ & 848 & 6.0 & 0.57 & 196 & 29.0 & 8.2 & 440 \\
\hline $16 \mathrm{~A}, \mathrm{~B}$ & 899 & 5.3 & 0.53 & 144 & 29.1 & 8.2 & 439 \\
\hline $17 \mathrm{~A}, \mathrm{~B}$ & 950 & 3.4 & 0.63 & 92 & 29.5 & 8.2 & 441 \\
\hline X06 & 985 & 2.5 & 0.33 & 92 & & & \\
\hline REW & 1,176 & 0.0 & 0.00 & & & & \\
\hline MEANS & & 8.0 & 0.93 & & & & \\
\hline TOTAL & 1,176 & & & 8,746 & & & \\
\hline
\end{tabular}




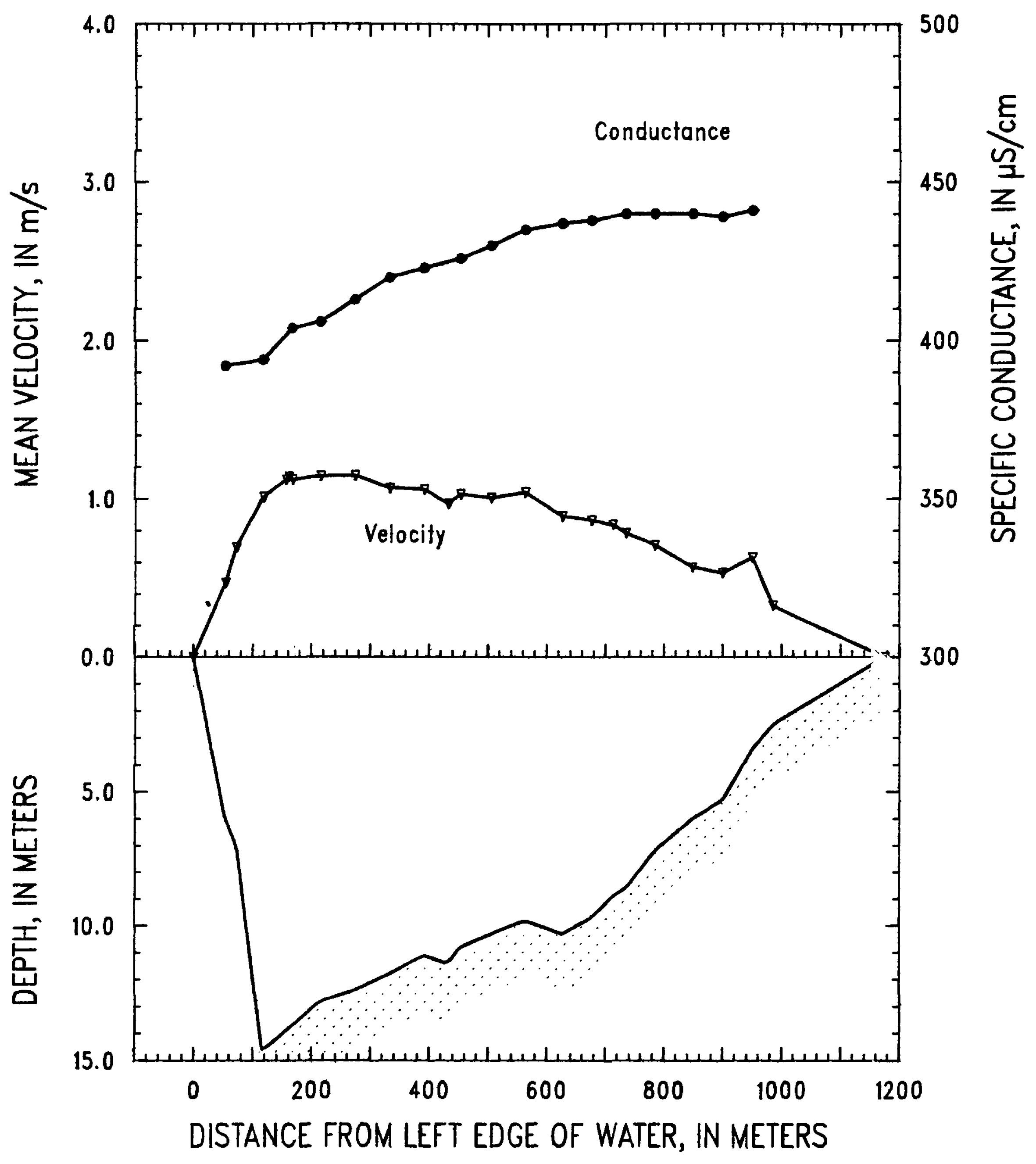

Figure 20. Mississippi River below Vicksburg, Mississippi, on August 3, 1991. 
SITE: Mississippi River near St. Francisville, La.-Mile 266.4

08-05-91

PARTY: Moody, Ellis, and LeBoeuf

GAGE HEIGHT @ Red River Landing: 19.6 ft GAGE HEIGHT @ Baton Rouge: $7.8 \mathrm{ft}$

RIVER SLOPE: $30 \times 10^{-6}$

SUSP. Bag sampler and 150 -lb weight

PRICE AA CURRENT METER No: W-223906 DATE RATED: 06-91

CURRENT METER EQUATION: $\mathrm{V}(\mathrm{m} / \mathrm{s})=\mathrm{Rev} / \mathrm{s}^{*} 0.670+0.010$

REMARKS:

Transit rate was $6.5 \mathrm{~cm} / \mathrm{s}$ and the nozzle was $3 / 16$ inch. Temperature, $\mathrm{pH}$, and specific cond rctance are the average of three separate samples.

\begin{tabular}{|c|c|c|c|c|c|c|c|}
\hline Vertical & $\begin{array}{l}\text { Distance } \\
\text { from } \\
\text { LEW } \\
\text { (m) }\end{array}$ & $\begin{array}{c}\text { Depth } \\
\text { (m) }\end{array}$ & $\begin{array}{l}\text { Mean } \\
\text { velocity } \\
(\mathrm{m} / \mathrm{s})\end{array}$ & $\begin{array}{c}\text { Discharge } \\
\left(\mathrm{m}^{3} / \mathrm{s}\right)\end{array}$ & $\begin{array}{c}\text { Temperature } \\
\left({ }^{\circ} \mathrm{C}\right)\end{array}$ & pH & $\begin{array}{c}\text { Speciflc } \\
\text { conductance } \\
(\mu \mathrm{S} / \mathrm{cm})\end{array}$ \\
\hline LEW & 0 & 0.0 & & & & & \\
\hline $01 \mathrm{~A}, \mathrm{~B}$ & 59 & 3.0 & 0.39 & 69 & 30.4 & 8.0 & 417 \\
\hline 02A,B & 118 & 5.5 & 0.56 & 159 & 30.5 & 8.0 & 417 \\
\hline $\mathrm{X} 05$ & 162 & 5.7 & 0.55 & 116 & -- & - & -- \\
\hline 03A,B & 192 & 5.6 & 0.59 & 125 & 30.2 & 8.0 & 417 \\
\hline 04A,B & 238 & 5.0 & 0.61 & 167 & 30.2 & 8.0 & 418 \\
\hline 05A,B & 301 & 5.9 & 0.74 & 272 & 30.2 & 8.0 & 418 \\
\hline 06A,B & 362 & 6.9 & 0.77 & 201 & 30.3 & 8.0 & 418 \\
\hline X04 & 377 & 7.0 & 0.76 & 161 & -- & - & -- \\
\hline 07A,B & 423 & 8.6 & 0.78 & 357 & 30.1 & 8.0 & 417 \\
\hline 08A,B & 484 & 8.5 & 0.80 & 421 & 30.1 & 8.0 & 417 \\
\hline 09A,B & 547 & 9.0 & 0.86 & 365 & 30.0 & 8.0 & 417 \\
\hline $\mathrm{X} 03$ & 578 & 9.9 & 0.92 & 199 & -- & -- & -- \\
\hline $10 \mathrm{~A}, \mathrm{~B}$ & 591 & 9.7 & 0.89 & 318 & 29.7 & 8.0 & 416 \\
\hline $11 \mathrm{~A}, \mathrm{~B}$ & 652 & 12.1 & 0.81 & 623 & 29.8 & 8.0 & 416 \\
\hline $12 A, B$ & 718 & 11.7 & 0.82 & 594 & 29.9 & 8.0 & 416 \\
\hline $13 \mathrm{~A}, \mathrm{~B}$ & 776 & 12.2 & 0.87 & 652 & 29.6 & 8.0 & 416 \\
\hline $14 A, B$ & 841 & 13.1 & 0.89 & 731 & 29.8 & 8.0 & 416 \\
\hline $15 \mathrm{~A}, \mathrm{~B}$ & 901 & 10.5 & 0.93 & 412 & 29.8 & 8.0 & 416 \\
\hline$X 01$ & 925 & 10.0 & 0.83 & 244 & -. & -. & - \\
\hline REW & 960 & 0.0 & & & & & \\
\hline MEANS & & 8.1 & 0.79 & & & & \\
\hline TOTAL & 960 & & & 6,186 & & & \\
\hline
\end{tabular}




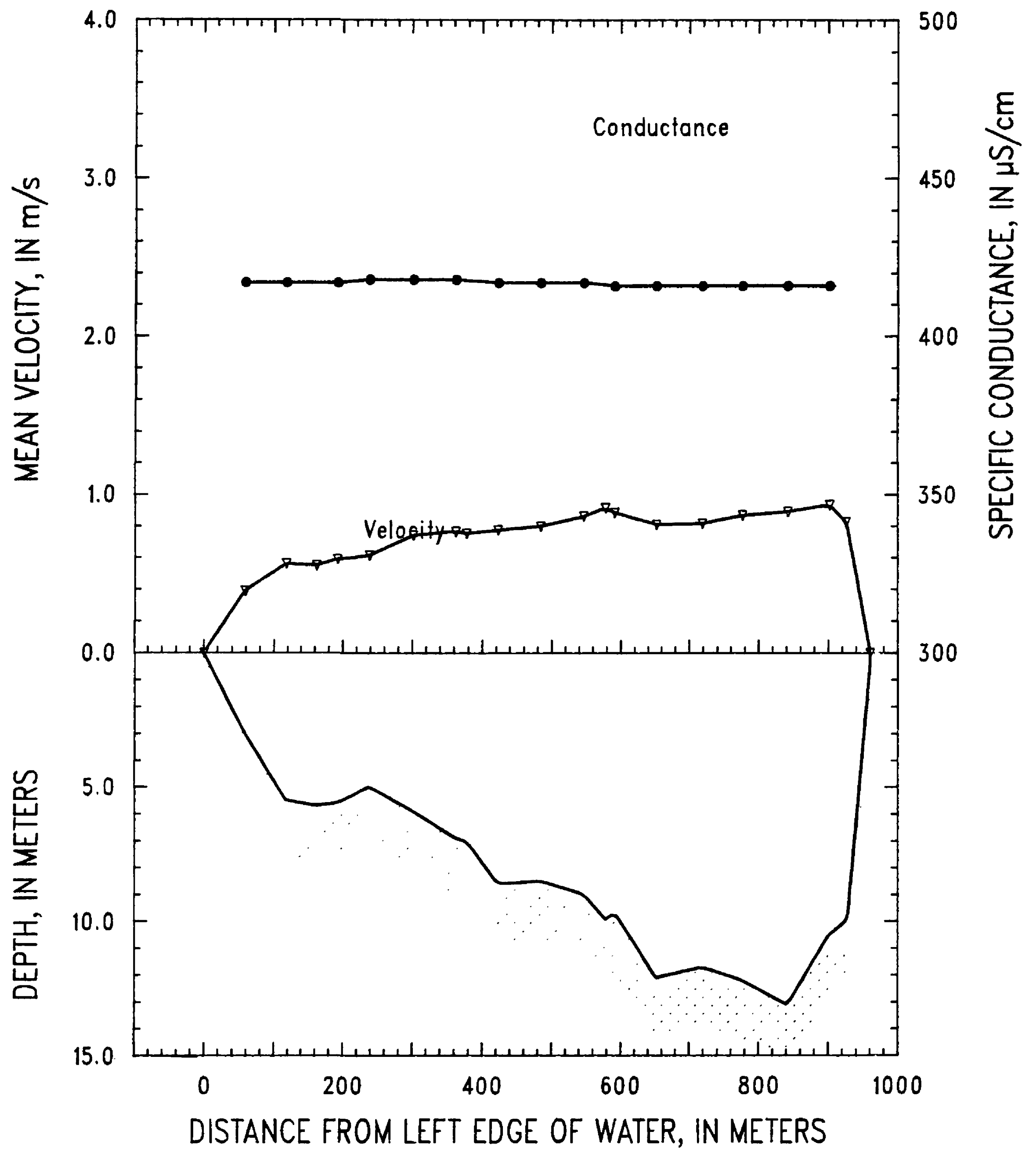

Figure 21. Mississippi River near St. Francisville, Louisiana, on August 5, 1991. 
SITE: Mississippi River below Belle Chasse, La.-Mile 72.8

08-07-91

PARTY: Moody, Ellis, and Simoneaux

GAGE HEIGHT @ Baton Rouge: 7.8 ft GAGE HEIGHT @ New Orleans: $\sim 2.9 \mathrm{ft}$

RIVER SLOPE: $7 \times 10^{-6}$

SUSP. Bag sampler and 150-lb weight

PRICE AA CURRENT METER No: W-223906 DATE RATED: 06-91

CURRENT METER EQUATION: $\mathrm{V}(\mathrm{m} / \mathrm{s})=\mathrm{Rev} / \mathrm{s}^{*} 0.670+0.010$

REMARKS:

Transit rate for velocity measurements was $3.0 \mathrm{~cm} / \mathrm{s}$. No depth-integrated sample was collected because of the slow water velocity. Since the water was well mixed, water was pumped from five equally sfaced verticals (2-6) for both the pump and depth-integrated sample. Temperature, $\mathrm{pH}$, and specific conductance are the average of three separate samples. Vertical 5 was measured after verticals 1-6.

\begin{tabular}{|c|c|c|c|c|c|c|c|}
\hline Vertical & $\begin{array}{l}\text { Distance } \\
\text { from } \\
\text { LEW } \\
\text { (m) }\end{array}$ & $\begin{array}{l}\text { Depth } \\
\text { (m) }\end{array}$ & $\begin{array}{c}\text { Moan } \\
\text { velocity } \\
(\mathrm{m} / \mathrm{s})\end{array}$ & $\begin{array}{c}\text { Discharge } \\
\left(\mathrm{m}^{3} / \mathrm{s}\right)\end{array}$ & $\begin{array}{l}\text { Temperature } \\
\left({ }^{\circ} \mathrm{C}\right)\end{array}$ & pH & $\begin{array}{c}\text { Spocific } \\
\text { conductance } \\
(\mu \mathrm{S} / \mathrm{cm})\end{array}$ \\
\hline LEW & 0 & 0.0 & 0.00 & & & & \\
\hline 06 & 84 & 10.2 & 0.21 & 233 & 30.2 & 7.9 & 465 \\
\hline 05 & 223 & 15.8 & 0.26 & 505 & 31.1 & 7.9 & 476 \\
\hline 04 & 326 & 20.2 & 0.31 & 748 & 30.3 & 7.9 & 468 \\
\hline 03 & 465 & 32.0 & 0.33 & 1,330 & 30.0 & 7.9 & 467 \\
\hline 02 & 581 & 35.1 & 0.33 & 1,110 & 30.2 & 7.9 & 465 \\
\hline 01 & 655 & 22.1 & 0.27 & 415 & 30.0 & 7.8 & 465 \\
\hline REW & 720 & 0.0 & 0.00 & & & & \\
\hline MEANS & & 20.1 & 0.30 & & & & \\
\hline TOTAL & 720 & & & 4,341 & & & \\
\hline
\end{tabular}




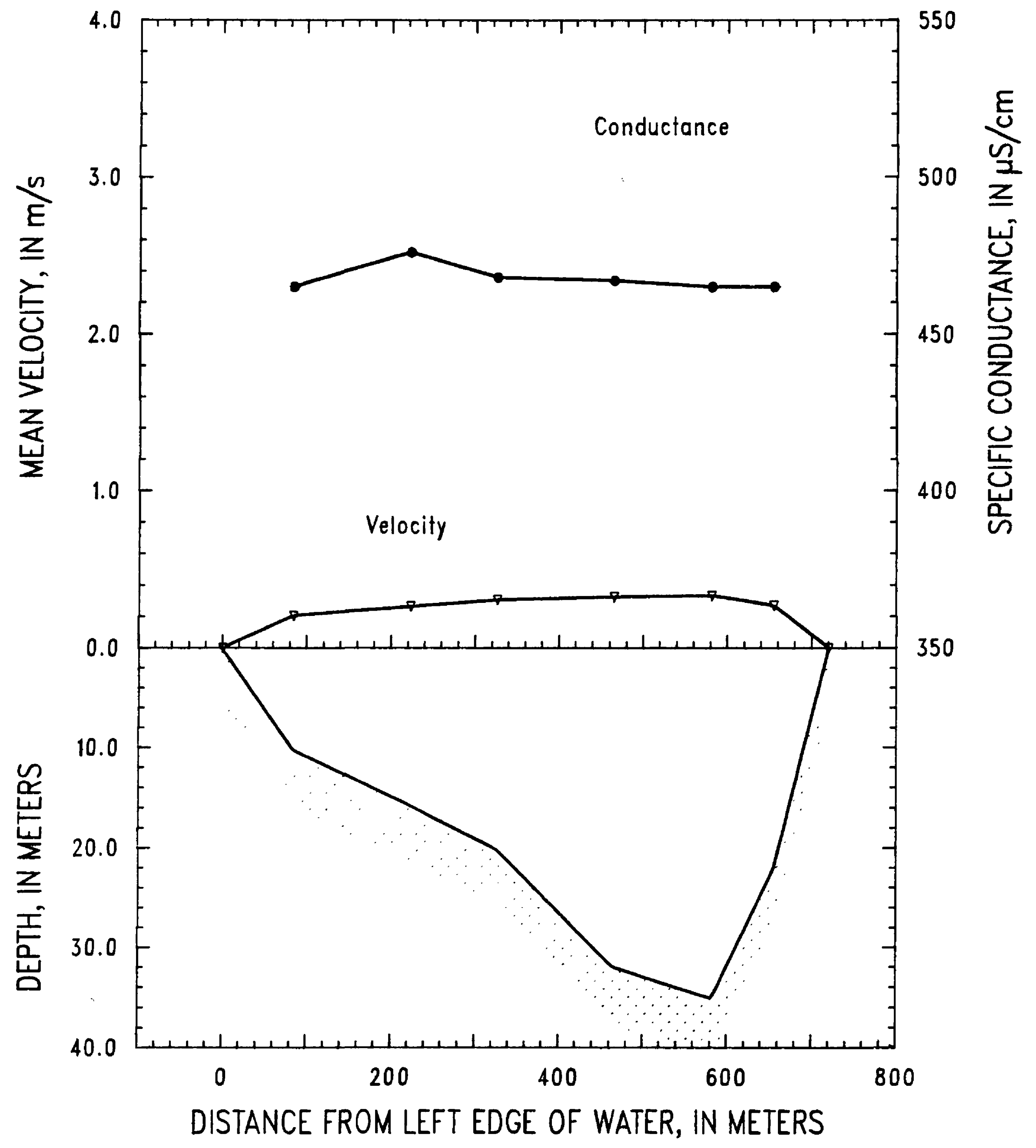

Figure 22. Mississippi River below Belle Chasse, Louisiana, on August 7, 1991. 
Bunter

84 


\section{DATA LISTINGS}

FOR

OCTOBER-NOVEMBER 1991 CRUISE 
SITE: Mississippi River above St. Anthony Falls, Minn.-Mile 857.7

$10-07-91$

PARTY: Moody, Noyes, Antweiler, and Simoneaux

GAGE HEIGHT @ Anoka: $808.57 \mathrm{ft}$ GAGE HEIGHT @ SAFU Pool: $799.70 \mathrm{ft}$

RIVER SLOPE: $156 \times 10^{-6}$

SUSP. Bag sampler and 150-lb weight

PRICE AA CURRENT METER No: W-223906 DATE RATED: 06-91

CURRENT METER EQUATION: $\mathrm{V}(\mathrm{m} / \mathrm{s})=\operatorname{Rev} / \mathrm{s}^{*} 0.670+0.010$

REMARKS:

Anchored at five verticals, used $5 / 16$-inch nozzle and $2.0 \mathrm{~cm} / \mathrm{s}$ transit rate. Temperature, $\mathrm{pH}$, and specific conductance were measured in order from left to right bank and are the average of three separate samples.

\begin{tabular}{|c|c|c|c|c|c|c|c|}
\hline Vertical & $\begin{array}{c}\text { Distance } \\
\text { from } \\
\text { LEW } \\
\text { (m) }\end{array}$ & $\begin{array}{l}\text { Depth } \\
\text { (m) }\end{array}$ & $\begin{array}{c}\text { Mean } \\
\text { velocity } \\
(\mathrm{m} / \mathrm{s})\end{array}$ & $\begin{array}{c}\text { Discharge } \\
\left(\mathrm{m}^{3} / \mathrm{s}\right)\end{array}$ & $\begin{array}{c}\text { Temperature } \\
\left({ }^{\circ} \mathrm{C}\right)\end{array}$ & pH & $\begin{array}{c}\text { Spechic } \\
\text { conductance } \\
(\mu \mathrm{S} / \mathrm{cm})\end{array}$ \\
\hline LEW & 0 & 0.0 & 0.00 & & & & \\
\hline $01 \mathrm{~A}, \mathrm{~B}$ & 27 & 2.4 & 0.59 & 30 & 10.0 & 8.4 & 443 \\
\hline$X 01$ & 42 & 3.3 & 0.46 & 14 & 10.3 & 8.4 & 446 \\
\hline $02 A, B$ & 46 & 3.2 & 0.51 & 24 & 10.2 & 8.5 & 451 \\
\hline$\times 02$ & 72 & 2.9 & 0.66 & 34 & 10.2 & 8.6 & 457 \\
\hline 03A, B & 81 & 3.5 & 0.55 & 20 & 10.2 & 8.6 & 461 \\
\hline$\times 03$ & 93 & 3.7 & 0.43 & 23 & 10.5 & 8.5 & 467 \\
\hline $04 A, B$ & 110 & 3.9 & 0.48 & 22 & 10.4 & 8.6 & 472 \\
\hline $05 \mathrm{~A}$ & 116 & 4.1 & 0.51 & 12 & -- & -- & -- \\
\hline 05B & 121 & 3.6 & 0.50 & 43 & 10.3 & 8.6 & 476 \\
\hline REW & 163 & 0.0 & 0.00 & & & & \\
\hline MEANS & & 2.6 & 0.52 & & & & \\
\hline TOTAL & 163 & & & 221 & & & \\
\hline
\end{tabular}




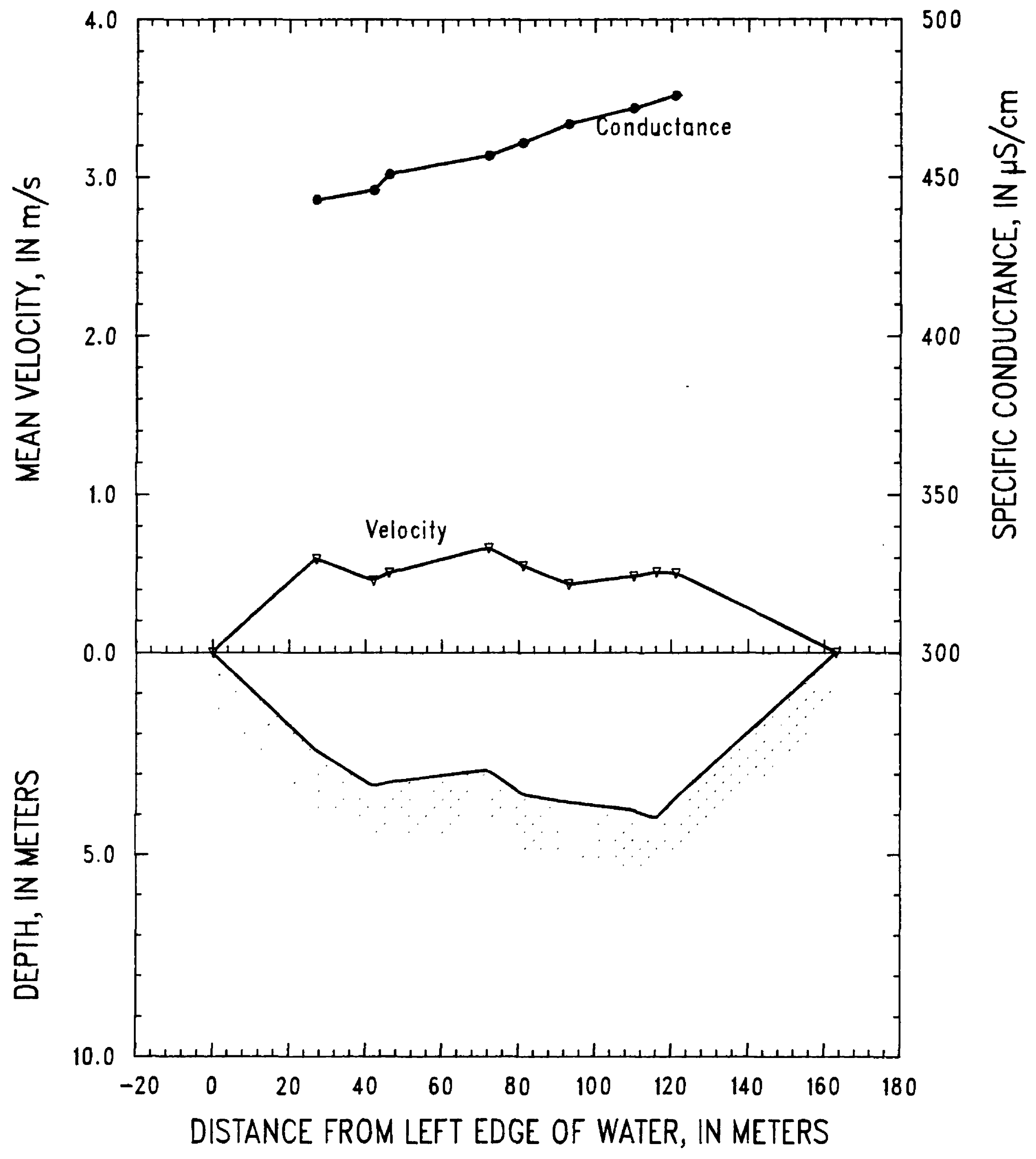

Figure 23. Mississippi River above St. Anthony Falls, Minnesota, cn October 7, 1991. 
SITE: Minnesota River at Mile 3.5, Minn.

10-08-91

PARTY: Moody, Antweiler, and Simoneaux

GAGE HEIGHT @ Savage: 688.7 ft GAGE HEIGHT @ Pool 2: $686.84 \mathrm{ft}$

RIVER SLOPE: $8 \times 10^{-6}$

SUSP. Bag sampler and 150-lb weight

PRICE AA CURRENT METER No: W-223906 DATE RATED: 06-91

CURRENT METER EQUATION: $V(\mathrm{~m} / \mathrm{s})=\operatorname{Rev} / \mathrm{s}^{*} 0.670+0.010$

REMARKS:

Anchored at five verticals, used $5 / 16$-inch nozzle and $2.0 \mathrm{~cm} / \mathrm{s}$ transit rate. Temperature, $\mathrm{pH}$, and specific conductance were collected from right to left bank.

\begin{tabular}{|c|c|c|c|c|c|c|c|}
\hline Vertical & $\begin{array}{c}\text { Distance } \\
\text { from } \\
\text { LEW } \\
\text { (m) }\end{array}$ & $\begin{array}{l}\text { Depth } \\
\text { (m) }\end{array}$ & $\begin{array}{l}\text { Mean } \\
\text { velocity } \\
(\mathrm{m} / \mathrm{s})\end{array}$ & $\begin{array}{c}\text { Discharge } \\
\left(\mathrm{m}^{3} / \mathrm{s}\right)\end{array}$ & $\begin{array}{c}\text { Temperature } \\
\left({ }^{\circ} \mathrm{C}\right)\end{array}$ & pH & $\begin{array}{c}\text { Specific } \\
\text { conductance } \\
(\mu \mathrm{S} / \mathrm{cm})\end{array}$ \\
\hline LEW & 0 & 0.0 & 0.00 & & & & \\
\hline $01 \mathrm{~A}$ & 17 & 3.7 & 0.28 & 16 & 11.3 & 8.5 & 927 \\
\hline $02 \mathrm{~A}$ & 31 & 4.7 & 0.34 & 31 & 11.5 & 8.5 & 928 \\
\hline 03A & 57 & 4.8 & 0.35 & 37 & 11.4 & 8.4 & 930 \\
\hline $04 \mathrm{~A}$ & 74 & 4.8 & 0.35 & 26 & 11.3 & 8.3 & 930 \\
\hline $05 \mathrm{~A}$ & 88 & 3.8 & 0.31 & 17 & 11.4 & 8.1 & 930 \\
\hline REW & 103 & 0.0 & 0.00 & & & & \\
\hline MEANS & & 3.7 & 0.33 & & & & \\
\hline TOTAL & 103 & & & 127 & & & \\
\hline
\end{tabular}




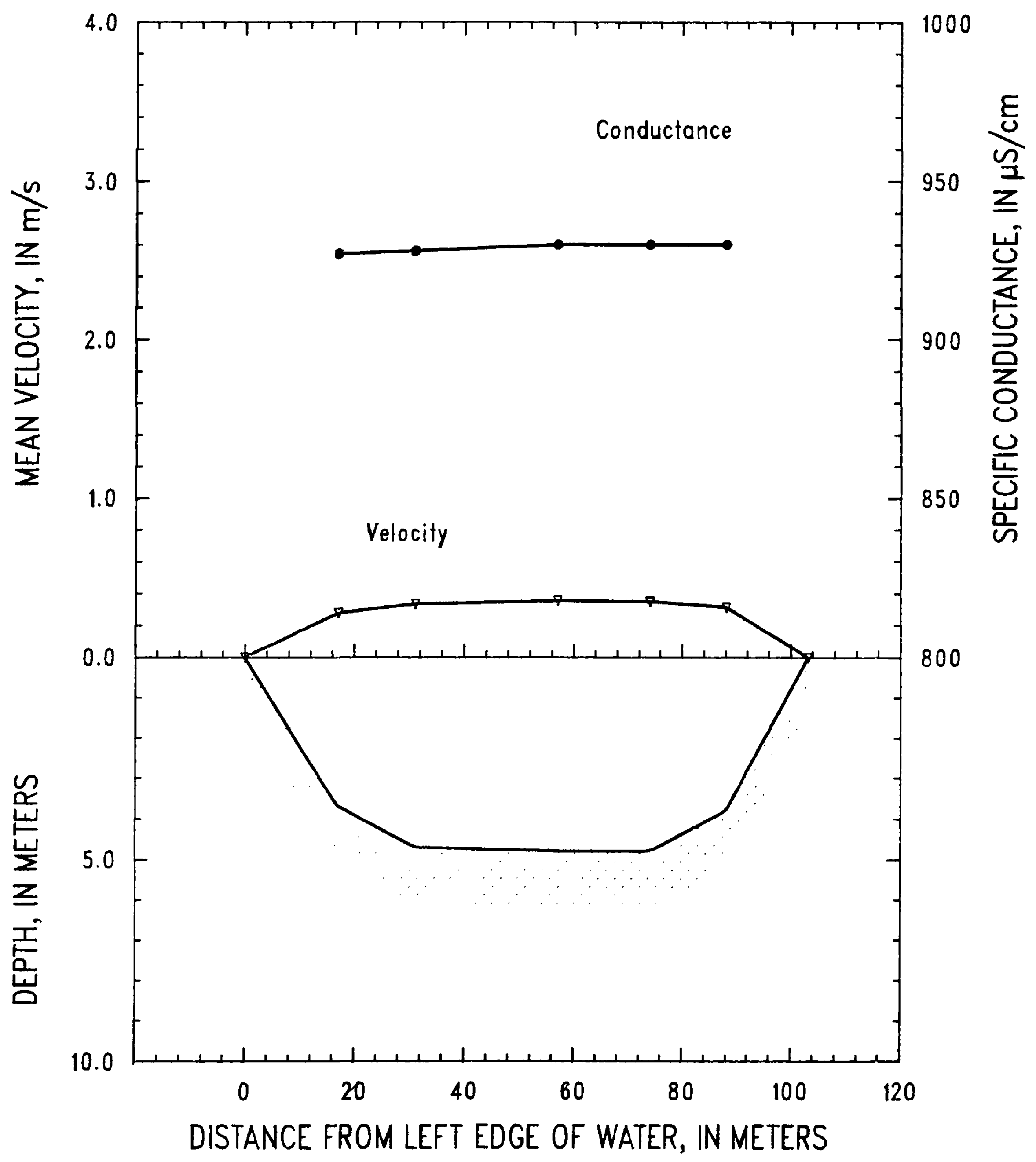

Figure 24. Minnesota River at Mile 3.5, Minnesota, on October 8, 1991. 
SITE: Mississippi River at Hastings, Minn.-Mile 812.2

PARTY: Moody and Simoneaux

GAGE HEIGHT @ TW Pool 2: $675.89 \mathrm{ft} \quad$ GAGE HEIGHT @ Pool 3: $674.10 \mathrm{ft}$

RIVER SLOPE: $19 \times 10^{-6}$

SUSP. Bag sampler and 150-lb weight

PRICE AA CURRENT METER No: W-223906 DATE RATED: 06-91

CURRENT METER EQUATION: $V(\mathrm{~m} / \mathrm{s})=\operatorname{Rev} / \mathrm{s}^{*} 0.670+0.010$

REMARKS:

Anchored at verticals 4 and 5. Did not collect a depth-integrated sample.

\begin{tabular}{|c|c|c|c|c|c|c|c|}
\hline Verticai & $\begin{array}{l}\text { Distance } \\
\text { from } \\
\text { LEW } \\
\text { (m) }\end{array}$ & $\begin{array}{l}\text { Depth } \\
\text { (m) }\end{array}$ & $\begin{array}{l}\text { Mean } \\
\text { velocity } \\
(\mathbf{m} / \mathbf{s})\end{array}$ & $\begin{array}{c}\text { Discharge } \\
\left(\mathrm{m}^{3} / \mathrm{s}\right)\end{array}$ & $\begin{array}{c}\text { Temperature } \\
\left({ }^{\circ} \mathrm{C}\right)\end{array}$ & pH & $\begin{array}{c}\text { Specific } \\
\text { conductance } \\
(\mu \mathrm{S} / \mathrm{cm})\end{array}$ \\
\hline LEW & 0 & 0.0 & 0.00 & & & & \\
\hline 01 & 36 & 7.5 & 0.50 & 145 & 11.0 & 8.5 & 653 \\
\hline 02 & 77 & 6.0 & 0.46 & 98 & 11.0 & 8.4 & 653 \\
\hline 03 & 107 & 5.1 & 0.38 & 69 & 11.1 & 8.4 & 653 \\
\hline 04 & 149 & 4.4 & 0.19 & 23 & 11.1 & 8.4 & 653 \\
\hline 05 & 162 & 4.0 & 0.11 & 15 & 11.1 & 8.3 & 654 \\
\hline REW & 215 & 0.0 & 0.00 & & & & \\
\hline MEANS & & 4.4 & 0.37 & & & & \\
\hline TOTAL & 215 & & & 350 & & & \\
\hline
\end{tabular}




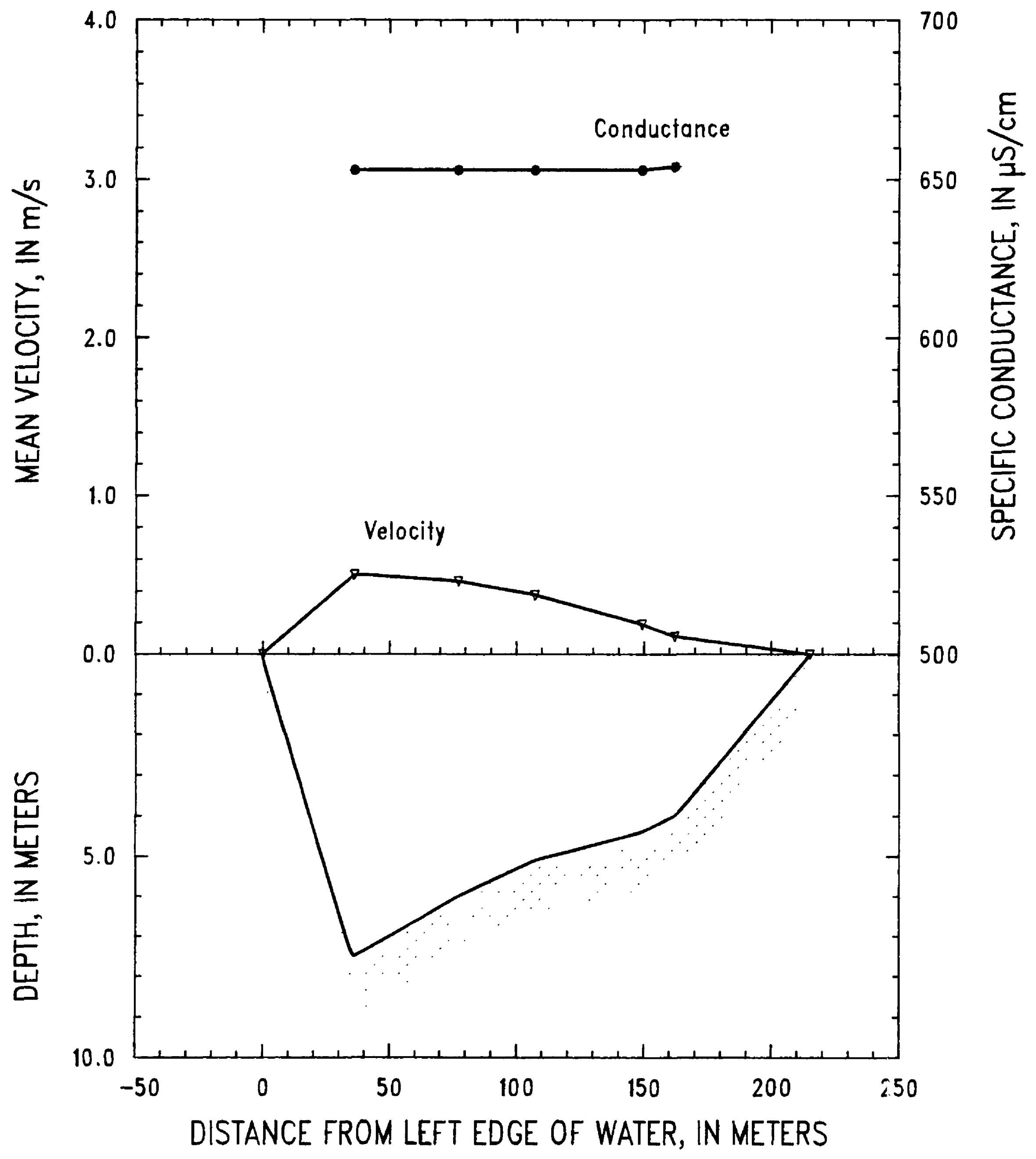

Figure 25. Mississippi River at Hastings, Minnesota, on October 10, 1991. 
SITE: Mississippi River near Pepin, Wis.-Mile 764.5

10-13-91

PARTY: Moody, Noyes, and Simoneaux

GAGE HEIGHT @ Lake City: 667.66 ft GAGE HEIGHT @ Wabasha: 667.11 ft

RIVER SLOPE: $8 \times 10^{-6}$

SUSP. Bag sampler and $150-$ lb weight

PRICE AA CURRENT METER No: W-223906 DATE RATED: 06-91

CURRENT METER EQUATION: $\mathrm{V}(\mathrm{m} / \mathrm{s})=\operatorname{Rev} / \mathrm{s}^{*} 0.670+0.010$

REMARKS:

Very strong wind (20-30 mph) upriver. No depth-integrated sample was collected. Vertical 1 at $55 \mathrm{~m}$ from LEW was not sampled because of the shallow water depths.

\begin{tabular}{|c|c|c|c|c|c|c|c|}
\hline Vertical & $\begin{array}{c}\text { Distance } \\
\text { from } \\
\text { LEW } \\
\text { (m) }\end{array}$ & $\begin{array}{l}\text { Depth } \\
\text { (m) }\end{array}$ & $\begin{array}{c}\text { Mean } \\
\text { veiocity } \\
(\mathbf{m} / \mathbf{s})\end{array}$ & $\begin{array}{c}\text { Dlscharge } \\
\left(\mathrm{m}^{3} / \mathrm{s}\right)\end{array}$ & $\begin{array}{l}\text { Temperature } \\
\left({ }^{\circ} \mathrm{C}\right)\end{array}$ & $\mathrm{pH}$ & $\begin{array}{c}\text { Specific } \\
\text { conductance } \\
(\mu \mathrm{S} / \mathrm{cm})\end{array}$ \\
\hline LEW & 0 & 0.0 & 0.00 & & & & \\
\hline 02 & 111 & 2.8 & 0.22 & 48 & 11.7 & 8.3 & 517 \\
\hline 03 & 157 & 2.9 & 0.23 & 36 & 11.9 & 8.3 & 515 \\
\hline 04 & 218 & 4.0 & 0.32 & 77 & 11.9 & 8.3 & 514 \\
\hline 05 & 278 & 4.0 & 0.35 & 65 & 11.9 & 8.3 & 514 \\
\hline $\mathrm{X} 01$ & 311 & 4.7 & 0.37 & 54 & -- & -- & - \\
\hline 06 & 340 & 5.6 & 0.38 & 54 & 12.0 & 8.3 & 514 \\
\hline X02 & 362 & 6.7 & 0.33 & 50 & -- & - & - \\
\hline 07 & 385 & 7.9 & 0.35 & 73 & 12.0 & 8.3 & 516 \\
\hline$X 03$ & 414 & 5.9 & 0.32 & 51 & -- & -- & -- \\
\hline REW & 440 & 0.0 & 0.00 & & & & \\
\hline MEANS & & 3.7 & 0.32 & & & & \\
\hline TOTAL & 440 & & & 508 & & & \\
\hline
\end{tabular}




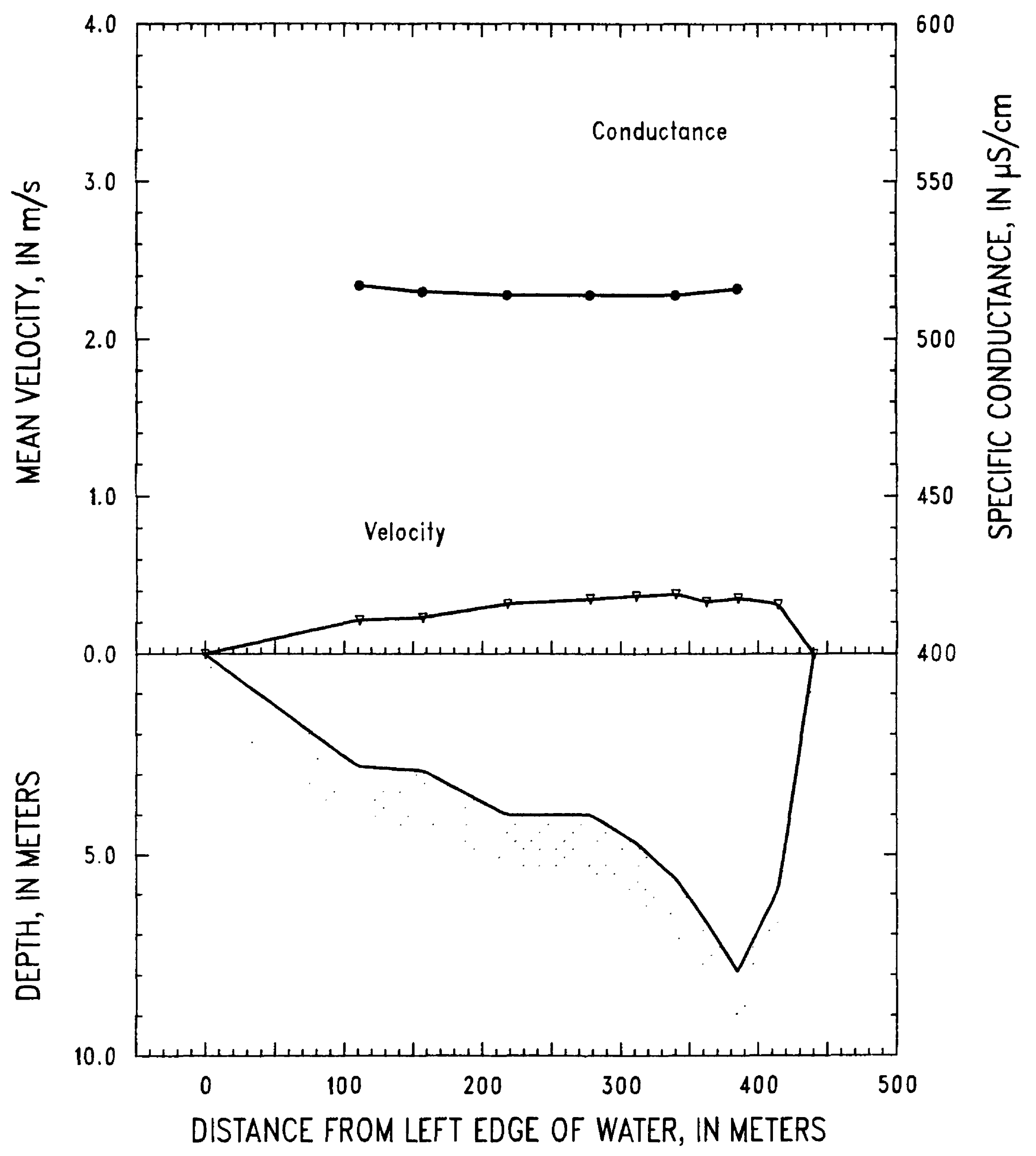

Figure 26. Mississippi River near Pepin, Wisconsin, on October 13, 1991. 
SITE: Mississippi River at Trempealeau, Wis.-713.8

10-15-91

PARTY: Moody, Bishop, and Simoneaux

GAGE HEIGHT @ Pool 6 TW: 639.98ft GAGE HEIGHT @ Pool 7: 639.15 ft

RIVER SLOPE: $14 \times 10^{-6}$

SUSP. Bag sampler and 150-lb weight

PRICE AA CURRENT METER No: W-223906 DATE RATED: 06-91

CURRENT METER EQUATION: $V(\mathrm{~m} / \mathrm{s})=\operatorname{Rev} / \mathrm{s}^{*} 0.670+0.010$

REMARKS:

No depth-integrated sample was collected. Vertical 1 at $48 \mathrm{~m}$ from LEW was not sampled because of the shallow water depths. Discharge at Dam 6 was reported to be $671 \mathrm{~m}^{3} / \mathrm{s}$.

\begin{tabular}{|c|c|c|c|c|c|c|c|}
\hline Vertical & $\begin{array}{l}\text { Dlstance } \\
\text { from } \\
\text { LEW } \\
\text { (m) }\end{array}$ & $\begin{array}{l}\text { Depth } \\
\text { (m) }\end{array}$ & $\begin{array}{c}\text { Mean } \\
\text { velocity } \\
(\mathrm{m} / \mathrm{s})\end{array}$ & $\begin{array}{c}\text { Discharge } \\
\left(\mathrm{m}^{3} / \mathrm{s}\right)\end{array}$ & $\begin{array}{c}\text { Temperature } \\
\left({ }^{\circ} \mathrm{C}\right)\end{array}$ & pH & $\begin{array}{c}\text { Speciflc } \\
\text { conductance } \\
(\mu \mathrm{S} / \mathrm{cm})\end{array}$ \\
\hline LEW & 0 & 0.0 & 0.00 & & & & \\
\hline 02 & 103 & 4.2 & 0.29 & 86 & 9.7 & 8.6 & 426 \\
\hline 03 & 143 & 4.8 & 0.24 & 49 & 9.8 & 8.5 & 427 \\
\hline 04 & 187 & 3.9 & 0.36 & 61 & 9.8 & 8.5 & 431 \\
\hline 05 & 229 & 4.0 & 0.42 & 80 & 9.7 & 8.5 & 431 \\
\hline 06 & 282 & 4.2 & 0.45 & 88 & 9.8 & 8.5 & 431 \\
\hline 07 & 322 & 5.5 & 0.45 & 119 & 9.8 & 8.4 & 435 \\
\hline 08 & 378 & 7.7 & 0.43 & 180 & 9.7 & 8.3 & 436 \\
\hline REW & 430 & 0.0 & 0.00 & & & & \\
\hline MEANS & & 4.0 & 0.38 & & & & \\
\hline TOTAL & 430 & & & 662 & & & \\
\hline
\end{tabular}




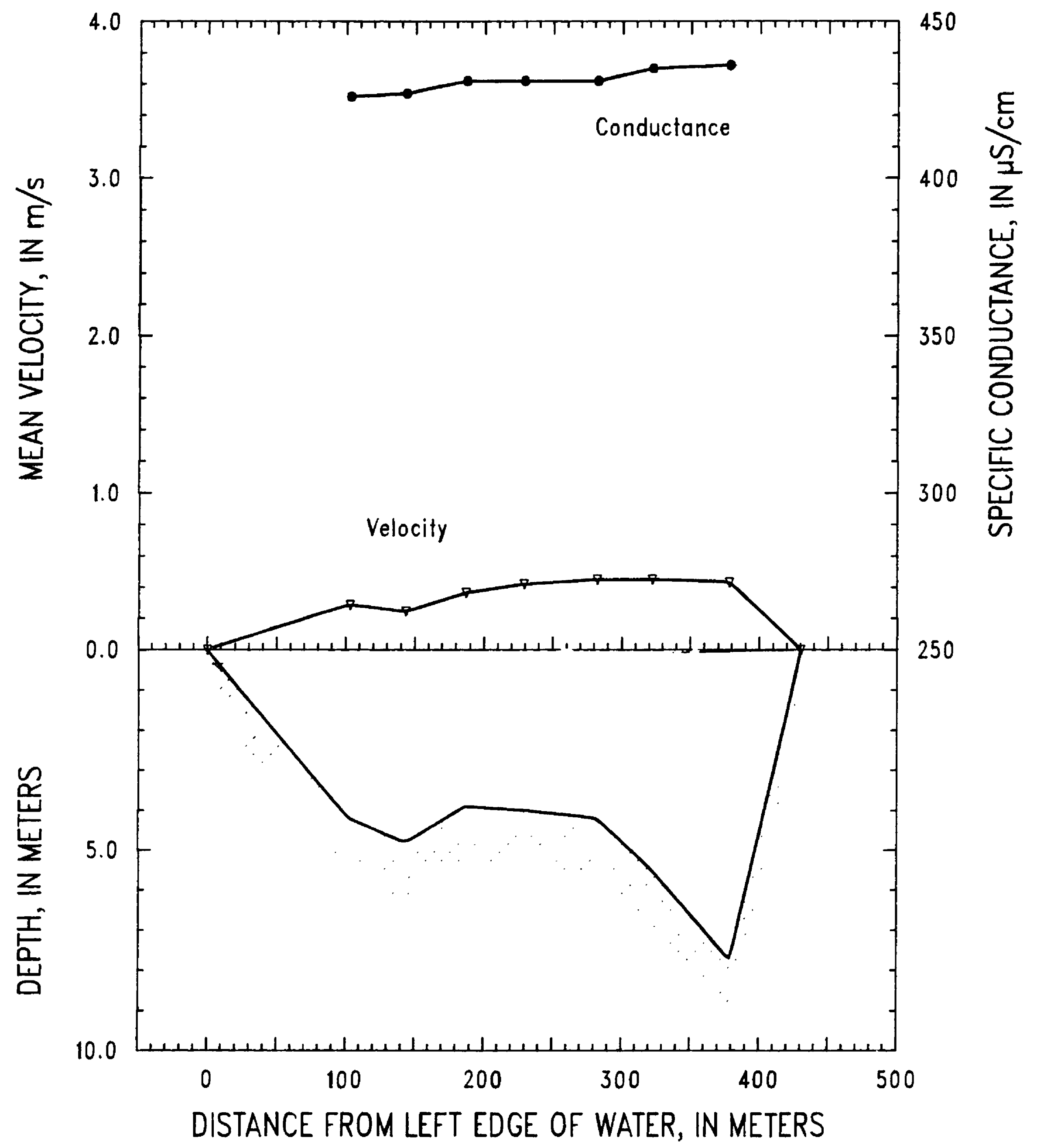

Figure 27. Mississippi River at Trempealeau, Wisconsin, on October 15, 1991. 
SITE: Mississippi River below Lock and Dam 9, Wis.--Mile 639.7

10-18-91

PARTY: Moody, Brinton, and Simoneaux

GAGE HEIGHT @ Pool 9 TW: 613.39 ft GAGE HEIGHT @ Pool 10: $611.18 \mathrm{ft}$

RIVER SLOPE: $12.7 \times 10^{-6}$

SUSP. Bag sampler and 150-lb weight

PRICE AA CURRENT METER No: W-223906 DATE RATED: 06-91

CURRENT METER EQUATTON: $V(\mathrm{~m} / \mathrm{s})=\operatorname{Rev} / \mathrm{s}^{*} 0.670+0.010$

REMARKS:

No depth-integrated sample was collected. Discharge from Dam 9 was reported to be $663 \mathrm{~m}^{3} / \mathrm{s}$. No sample was collected at vertical 1 because of the shallow water.

\begin{tabular}{|c|c|c|c|c|c|c|c|}
\hline Vertical & $\begin{array}{c}\text { Distance } \\
\text { from } \\
\text { LEW } \\
\text { (m) }\end{array}$ & $\begin{array}{l}\text { Depth } \\
\text { (m) }\end{array}$ & $\begin{array}{c}\text { Mean } \\
\text { velocity } \\
(\mathrm{m} / \mathrm{s})\end{array}$ & $\begin{array}{c}\text { Discharge } \\
\left(\mathrm{m}^{3} / \mathrm{s}\right)\end{array}$ & $\begin{array}{c}\text { Temperature } \\
\left({ }^{\circ} \mathrm{C}\right)\end{array}$ & pH & $\begin{array}{c}\text { Specific } \\
\text { conductance } \\
(\mu \mathrm{S} / \mathrm{cm})\end{array}$ \\
\hline LEW & 0 & 0.0 & 0.00 & & & & \\
\hline 02 & 107 & 3.6 & 0.32 & 97 & 10.7 & 8.5 & 436 \\
\hline 03 & 168 & 5.0 & 0.42 & 125 & 10.7 & 8.5 & 438 \\
\hline 04 & 226 & 5.9 & 0.42 & 126 & 11.0 & 8.5 & 439 \\
\hline 05 & 270 & 5.6 & 0.45 & 133 & 10.8 & 8.5 & 437 \\
\hline 06 & 332 & 5.2 & 0.43 & 119 & 10.6 & 8.5 & 439 \\
\hline 07 & 376 & 4.3 & 0.39 & 94 & 10.7 & 8.5 & 442 \\
\hline REW & 444 & 0.0 & 0.00 & & & & \\
\hline MEANS & & 3.9 & 0.40 & & & & \\
\hline TOTAL & 444 & & & 694 & & & \\
\hline
\end{tabular}




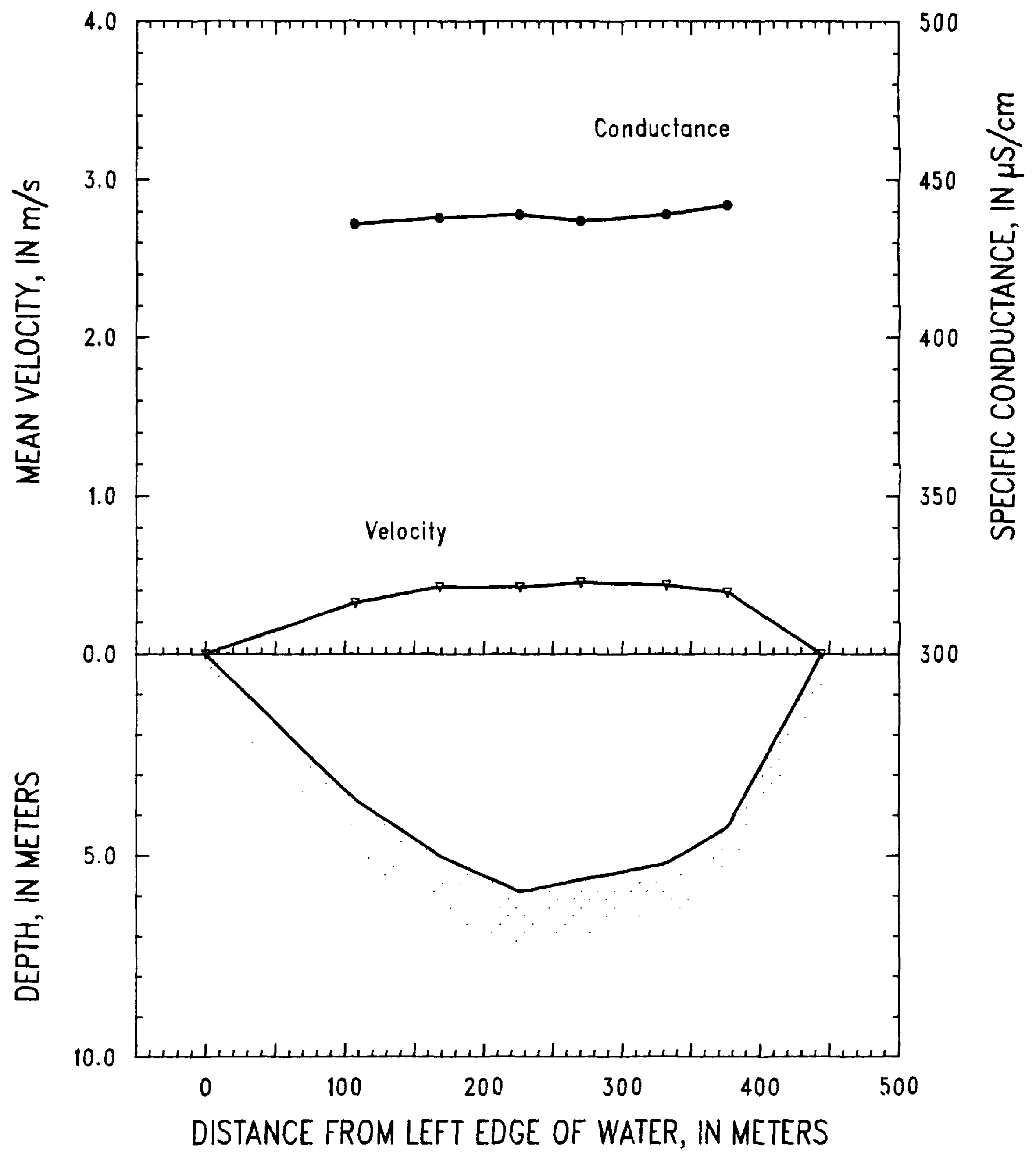

Figure 28. Mississippi River below Lock and Dam 9, Wisconsin, on October 18, 1991. 
SITE: Mississippi River at Clinton, Iowa-Mile 520.3

PARTY: Moody, Brinton, and Simoneaux

GAGE HEIGHT @ Dam 13 TW: $573.75 \mathrm{ft}$ GAGE HEIGHT @ Pool 14: $572.02 \mathrm{ft}$

RIVER SLOPE: $10.9 \times 10^{-6}$

SUSP. Bag sampler and 150-lb weight

PRICE AA CURRENT METER No: W-223906 DATE RATED: 06-91

CURRENT METER EQUATION: $V(\mathrm{~m} / \mathrm{s})=\operatorname{Rev} / \mathrm{s} * 0.670+0.010$

REMARKS:

No depth-integrated sample was collected. Vertical 1 at $56 \mathrm{~m}$ from LEW was not sampled because of the shallow water depths. Doug Goodrich (USGS, Iowa City) measured a discharge of $880 \mathrm{~m}^{3} / \mathrm{s}$ from the Highway 30 bridge.

\begin{tabular}{|c|c|c|c|c|c|c|c|}
\hline Vertical & $\begin{array}{l}\text { Distance } \\
\text { from } \\
\text { LEW } \\
\text { (m) }\end{array}$ & $\begin{array}{l}\text { Depth } \\
\text { (m) }\end{array}$ & $\begin{array}{c}\text { Mean } \\
\text { velocity } \\
(\mathrm{m} / \mathrm{s})\end{array}$ & $\begin{array}{c}\text { Discharge } \\
\left(\mathrm{m}^{3} / \mathrm{s}\right)\end{array}$ & $\begin{array}{c}\text { Temperature } \\
\left({ }^{\circ} \mathrm{C}\right)\end{array}$ & pH & $\begin{array}{c}\text { Speclfic } \\
\text { conductance } \\
(\mu \mathrm{S} / \mathrm{cm})\end{array}$ \\
\hline LEW & 0 & 0.0 & 0.00 & & & & \\
\hline 02 & 115 & 4.0 & 0.18 & 58 & 11.9 & 8.5 & 425 \\
\hline 03 & 165 & 6.6 & 0.31 & 107 & 12.0 & 8.5 & 423 \\
\hline 04 & 221 & 7.1 & 0.39 & 150 & 11.7 & 8.5 & 425 \\
\hline 05 & 274 & 8.2 & 0.43 & 211 & 12.2 & 8.5 & 427 \\
\hline 06 & 340 & 8.9 & 0.44 & 231 & 12.1 & 8.4 & 428 \\
\hline 07 & 392 & 8.3 & 0.40 & 182 & 12.2 & 8.4 & 426 \\
\hline REW & 450 & 0.0 & 0.00 & & & & \\
\hline MEANS & & 5.6 & 0.37 & & & & \\
\hline TOTAL & 450 & & & 939 & & & \\
\hline
\end{tabular}




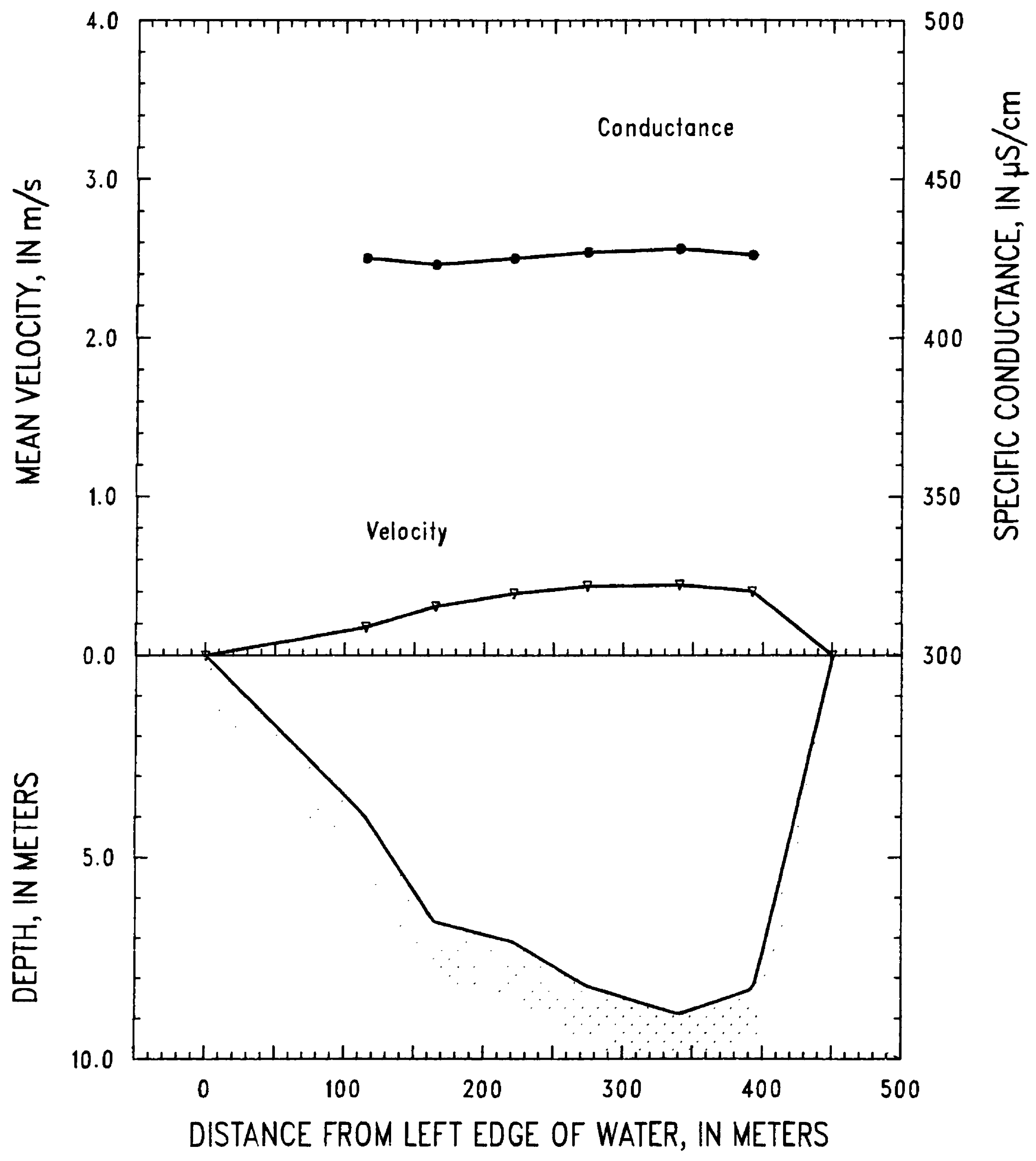

Figure 29. Mississippi River at Clinton, lowa, on October 22, 1991. 
SITE: Mississippi River at Keokuk, Iowa-Mile 363.1

$10-27-91$

PARTY: Moody, Garbarino, and LeBoeuf

GAGE HEIGHT @ Dam 19 TW: $481.65 \mathrm{ft}$ GAGE HEIGHT @ Pool 20: $479.38 \mathrm{ft}$

RIVER SLOPE: $20.5 \times 10^{-6}$

SUSP. Bag sampler and 150-lb weight

PRICE AA CURRENT METER No: W-223906 DATE RATED: 06-91

CURRENT METER EQUATION: $V(\mathrm{~m} / \mathrm{s})=\operatorname{Rev} / \mathrm{s}^{*} 0.670+0.010$

REMARKS:

No depth-in tegrated sample was collected. Water level rose about $0.3 \mathrm{~m}$ after starting section. Mean discharge from Dam 19 was about $1,300 \mathrm{~m}^{3} / \mathrm{s}$.

\begin{tabular}{lccccccc}
\hline Vertical & $\begin{array}{c}\text { Distance } \\
\text { from } \\
\text { LEW } \\
(\mathrm{m})\end{array}$ & $\begin{array}{c}\text { Depth } \\
(\mathrm{m})\end{array}$ & $\begin{array}{c}\text { Mean } \\
\text { velocity } \\
(\mathrm{m} / \mathrm{s})\end{array}$ & $\begin{array}{c}\text { Dlscharge } \\
\left(\mathrm{m}^{3} / \mathrm{s}\right)\end{array}$ & $\begin{array}{c}\text { Temperature } \\
\left({ }^{\circ} \mathrm{C}\right)\end{array}$ & pH & $\begin{array}{c}\text { Specific } \\
\text { conductance } \\
(\mu \mathrm{S} / \mathrm{cm})\end{array}$ \\
\hline LEW & 0 & 0.0 & 0.00 & & & & \\
01 & 96 & 2.6 & 0.38 & 95 & 14.5 & 8.3 & 458 \\
02 & 193 & 3.4 & 0.39 & 126 & 14.0 & 8.3 & 459 \\
03 & 287 & 3.0 & 0.64 & 173 & 14.0 & 8.4 & 460 \\
04 & 374 & 3.2 & 0.70 & 204 & 13.9 & 8.4 & 462 \\
05 & 470 & 4.3 & 0.83 & 345 & 13.9 & 8.4 & 462 \\
06 & 567 & 4.1 & 0.79 & 287 & 14.0 & 8.4 & 464 \\
07 & 647 & 3.2 & 0.63 & 178 & 13.9 & 8.4 & 463 \\
REW & 745 & 0.0 & 0.00 & & & & \\
MEANS & & 3.0 & 0.64 & & & & \\
TOTAL & 745 & & & 1,408 & & & \\
\hline
\end{tabular}




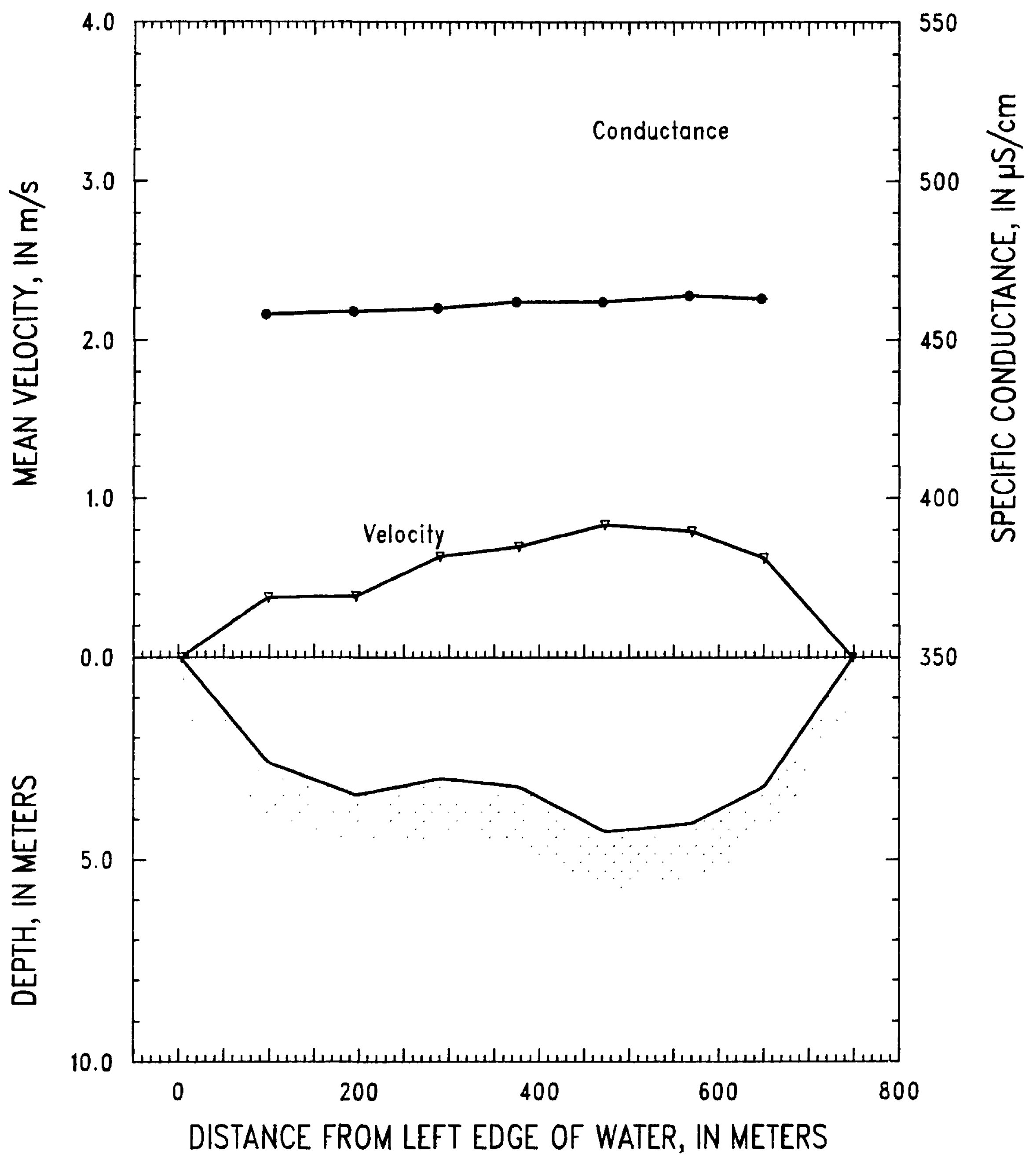

Figure 30. Mississippi River at Keokuk, lowa, on October 27, 1991. 
SITE: Mississippi River near Winfield, Mo.-Mile 239.2

PARTY: Moody, Garbarino, and Simoneaux

GAGE HEIGHT @ Dam 25 TW: 421.28 ft GAGE HEIGHT @ Pool 26: $419.10 \mathrm{ft}$

RIVER SLOPE: $10.3 \times 10^{-6}$

SUSP. Bag sampler and 150-lb weight

PRICE AA CURRENT METER No: W-223906 DATE RATED: 06-91

CURRENT METER EQUATION: $V(\mathrm{~m} / \mathrm{s})=\operatorname{Rev} / \mathrm{s}^{*} 0.670+0.010$

REMARKS:

No depth-integrated sample was collected.

\begin{tabular}{lccccccc}
\hline Vertical & $\begin{array}{c}\text { Distance } \\
\text { from } \\
\text { LEW } \\
(\mathbf{m})\end{array}$ & $\begin{array}{c}\text { Depth } \\
(\mathbf{m})\end{array}$ & $\begin{array}{c}\text { Mean } \\
\text { velocity } \\
(\mathbf{m} / \mathbf{s})\end{array}$ & $\begin{array}{c}\text { Discharge } \\
\left(\mathbf{m}^{3} / \mathbf{s}\right)\end{array}$ & $\begin{array}{c}\text { Temperature } \\
\left({ }^{\circ} \mathbf{C}\right)\end{array}$ & pH & $\begin{array}{c}\text { Specific } \\
\text { conductance } \\
(\mu \mathbf{S} / \mathbf{c m})\end{array}$ \\
\hline LEW & 0 & 0.0 & 0.00 & & & & \\
01 & 93 & 7.7 & 0.55 & 283 & 13.3 & 8.4 & 453 \\
02 & 135 & 7.6 & 0.55 & 261 & 13.4 & 8.4 & 454 \\
03 & 218 & 6.9 & 0.52 & 269 & 13.5 & 8.4 & 454 \\
04 & 284 & 5.2 & 0.48 & 167 & 13.6 & 8.4 & 454 \\
05 & 352 & 4.7 & 0.35 & 111 & 13.6 & 8.4 & 454 \\
06 & 419 & 4.1 & 0.35 & 78 & 13.5 & 8.4 & 453 \\
07 & 461 & 3.2 & 0.28 & 64 & 13.5 & 8.4 & 453 \\
REW & 563 & 0.0 & 0.00 & & & & \\
MEANS & & 4.7 & 0.47 & & & & \\
TOTAL & 563 & & & 1,233 & & & \\
\hline
\end{tabular}




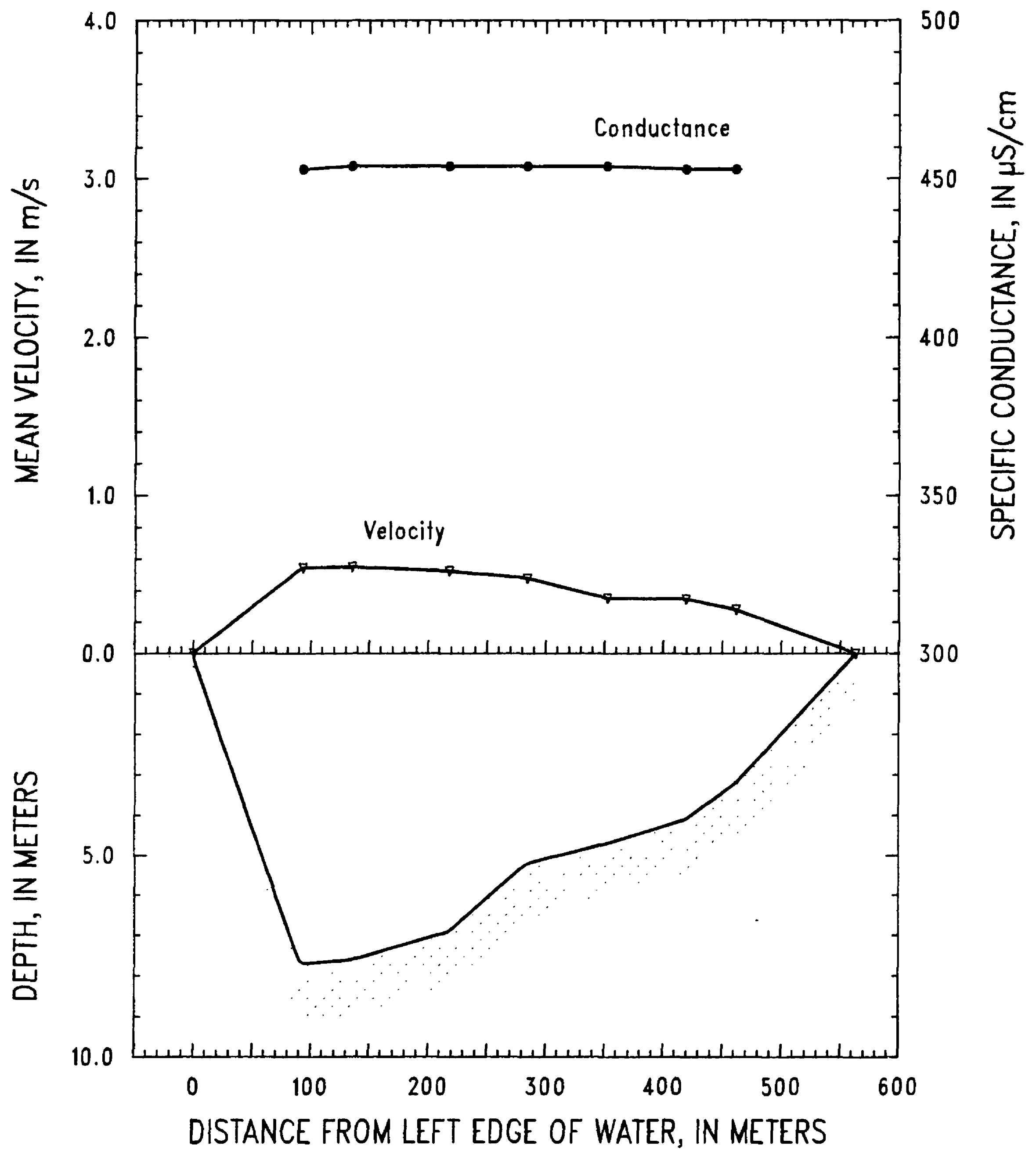

Figure 31. Mississippi River near Winfield, Missouri, on October 30, 1991. 
SITE: Illinois River at Hardin, Ill.-Mile 21.8

10-31-91

PARTY: Moody, Garbarino, Krest, and Simoneaux

GAGE HEIGHT @ Meredosia: $442.1 \mathrm{ft}$ GAGE HEIGHT @ Grafton: $419.3 \mathrm{ft}$

RIVER SLOPE: $60.3 \times 10^{-6}$

SUSP. 15-lb weight

PRICE AA CURRENT METER No: W-223906 DATE RATED: 06-91

CURRENT METER EQUATION: $V(\mathrm{~m} / \mathrm{s})=\operatorname{Rev} / \mathrm{s}^{*} 0.670+0.010$

REMARKS:

Velocities were measured from the small boat at 0.6 depth and by depth integration from the small boat. One trisponder was at LEW and one was in the small boat. A discharge of $525 \mathrm{~m}^{3} / \mathrm{s}$ was calculared using the 0.6 depth velocity measurements.

\begin{tabular}{|c|c|c|c|c|c|c|c|}
\hline Verticai & $\begin{array}{l}\text { Distance } \\
\text { from } \\
\text { LEW } \\
\text { (m) }\end{array}$ & $\begin{array}{l}\text { Depth } \\
\text { (m) }\end{array}$ & $\begin{array}{l}\text { Mean } \\
\text { velocity } \\
(\mathrm{m} / \mathrm{s})\end{array}$ & $\begin{array}{c}\text { Discharge } \\
\left(\mathrm{m}^{3} / \mathrm{s}\right)\end{array}$ & $\begin{array}{c}\text { Temperature } \\
\left.\text { ( }{ }^{\circ} \mathrm{C}\right)\end{array}$ & pH & $\begin{array}{c}\text { Speclific } \\
\text { conductance } \\
(\mu \mathrm{S} / \mathrm{cm})\end{array}$ \\
\hline LEW & 0 & 0.0 & 0.00 & & & & \\
\hline 01 & 23 & 1.3 & 0.19 & 7 & 13.8 & 8.0 & 671 \\
\hline 02 & 53 & 3.4 & 0.33 & 42 & 13.9 & 8.0 & 673 \\
\hline 03 & 97 & 4.1 & 0.47 & 81 & 13.7 & 8.0 & 673 \\
\hline 09 & 138 & 4.8 & 0.56 & 68 & 13.6 & 8.0 & 676 \\
\hline 04 & 148 & 5.2 & 0.59 & 52 & 13.8 & 8.0 & 676 \\
\hline 05 & 172 & 6.1 & 0.53 & 87 & 14.0 & 8.0 & 676 \\
\hline 06 & 202 & 6.1 & 0.49 & 99 & 13.9 & 8.0 & 678 \\
\hline 07 & 238 & 5.8 & 0.44 & 63 & 13.8 & 8.0 & 678 \\
\hline 08 & 251 & 4.0 & 0.33 & 21 & 13.7 & 8.0 & 675 \\
\hline REW & 270 & 0.0 & 0.00 & & & & \\
\hline MEANS & & 4.1 & 0.47 & & & & \\
\hline TOTAL & 270 & & & 520 & & & \\
\hline
\end{tabular}




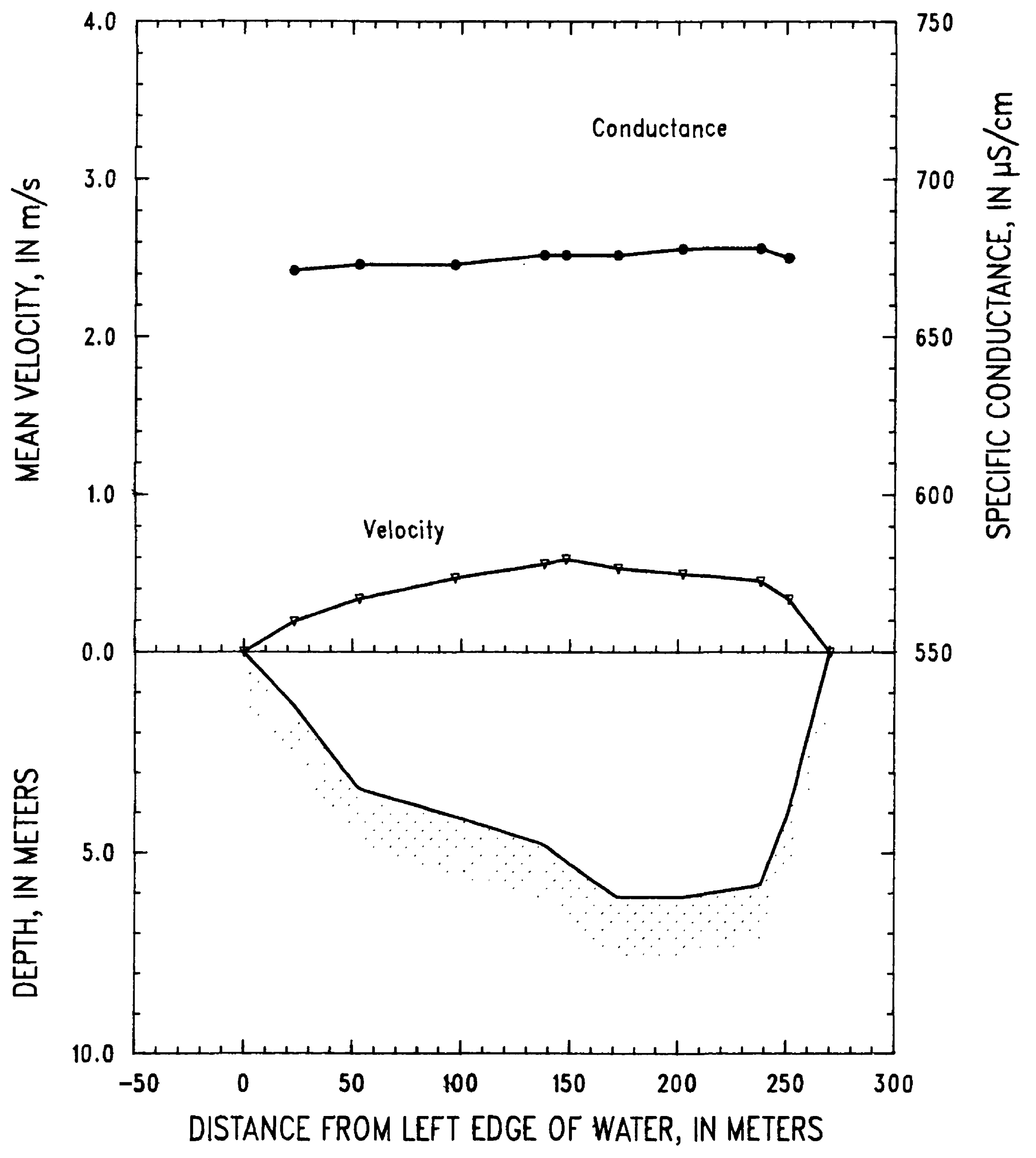

Figure 32. Illinois River at Hardin, Illinois, on October 31, 1991. 
SITE: Missouri River at St. Charles, Mo.-Mile 24.8

PARTY: Moody, Roth, and Simoneaux

GAGE HEIGHT @ Hermann: 488.56 ft GAGE HEIGHT @ St. Charles: $432.0 \mathrm{ft}$

RIVER SLOPE: $151 \times 10^{-6}$

SUSP. Bag sampler and 150-lb weight

PRICE AA CURRENT METER No: W-223906 DATE RATED: 06-91

CURRENT METER EQUATION: $\mathrm{V}(\mathrm{m} / \mathrm{s})=\operatorname{Rev} / \mathrm{s}^{*} 0.670+0.010$

REMARKS:

Several problems were encountered because of the very cold weather $\left(-1\right.$ to $\left.2^{\circ} \mathrm{C}\right)$. Transit rate was $5 \mathrm{~cm} / \mathrm{s}$ and nozzle size was $1 / 4$ inch. Unmeasured zone was $0.67 \mathrm{~m}$. Gage heights above are for November 2, 1991.

\begin{tabular}{|c|c|c|c|c|c|c|c|}
\hline Vertical & $\begin{array}{c}\text { Distance } \\
\text { from } \\
\text { LEW } \\
\text { (m) }\end{array}$ & $\begin{array}{l}\text { Depth } \\
\text { (m) }\end{array}$ & $\begin{array}{c}\text { Mean } \\
\text { velocity } \\
(\mathrm{m} / \mathrm{s})\end{array}$ & $\begin{array}{c}\text { Discharge } \\
\left(\mathrm{m}^{3} / \mathrm{s}\right)\end{array}$ & $\begin{array}{c}\text { Temperature } \\
\left({ }^{\circ} \mathrm{C}\right)\end{array}$ & pH & $\begin{array}{c}\text { Specific } \\
\text { conductance } \\
(\mu \mathrm{S} / \mathrm{cm})\end{array}$ \\
\hline LEW & 0 & 0.0 & 0.00 & & & & \\
\hline 01A,B & 39 & 6.0 & 1.07 & 187 & 7.8 & 8.0 & 655 \\
\hline 02A,B & 58 & 4.9 & 1.15 & 105 & 7.9 & 8.0 & 654 \\
\hline 03A,B & 76 & 4.7 & 1.21 & 123 & 7.9 & 8.0 & 654 \\
\hline 04A,B & 101 & 4.6 & 1.22 & 146 & 8.0 & 8.0 & 652 \\
\hline 05A,B & 128 & 4.5 & 1.10 & 137 & 8.1 & 8.0 & 652 \\
\hline 06A,B & 156 & 4.2 & 1.20 & 123 & 8.1 & 8.0 & 654 \\
\hline 07A,B & 177 & 4.3 & 1.16 & 113 & 8.1 & 8.0 & 656 \\
\hline 08A,B & 201 & 4.7 & 1.12 & 131 & 8.3 & 8.0 & 658 \\
\hline 09A,B & 227 & 4.6 & 1.16 & 141 & 8.2 & 7.9 & 658 \\
\hline $10 \mathrm{~A}, \mathrm{~B}$ & 254 & 4.7 & 1.19 & 148 & 8.2 & 7.8 & 659 \\
\hline REW & 280 & 0.0 & 0.00 & & & & \\
\hline MEANS & & 4.2 & 1.15 & & & & \\
\hline TOTAL & 280 & & & 1,352 & & & \\
\hline
\end{tabular}




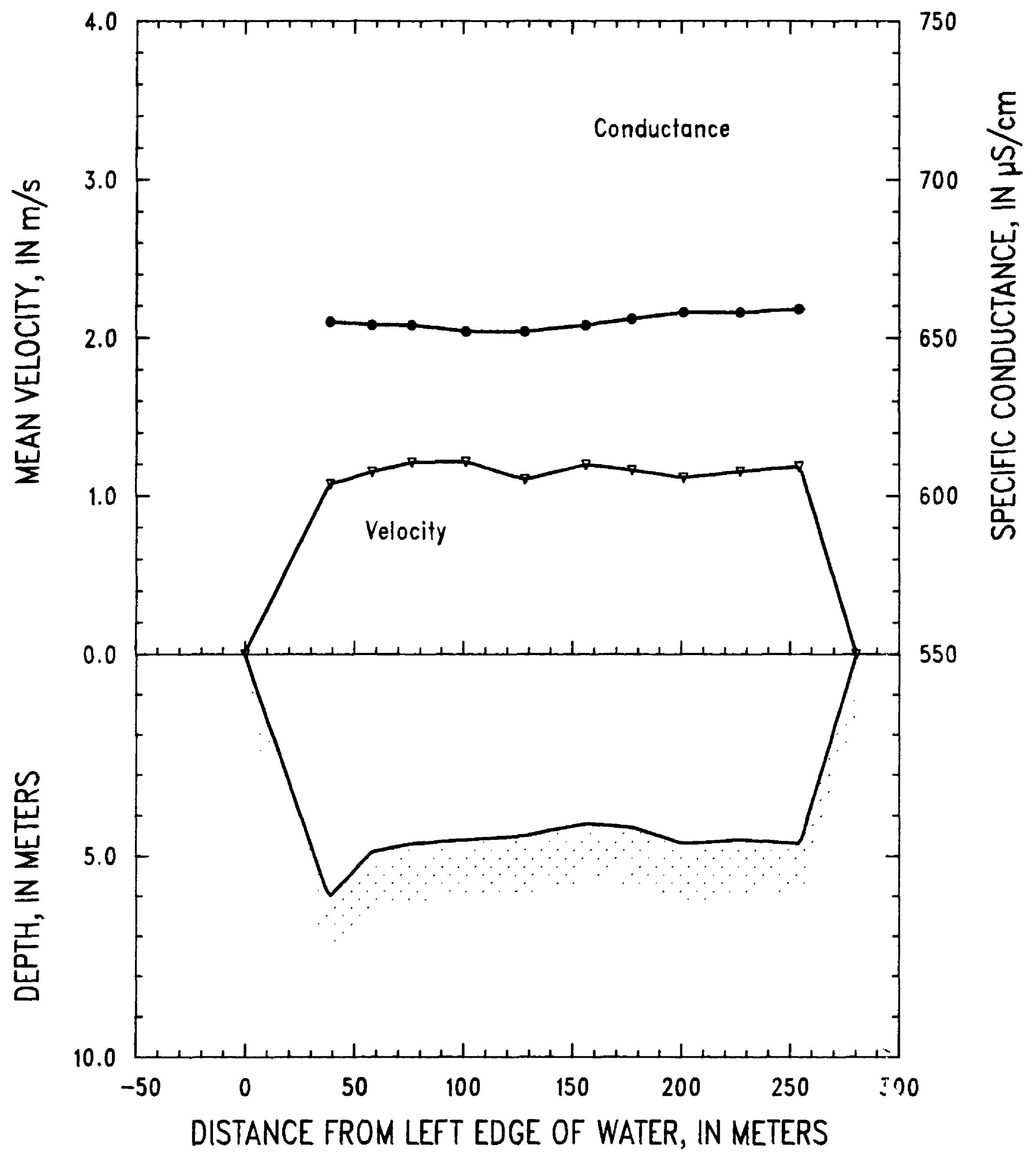

Figure 33. Missouri River at St. Charles, Missouri, on November 3, 1991. 
SITE: Mississippi River at Thebes, Ill--Mile 44.0

$11-05-91$

PARTY: Moody, Roth, and Simoneaux

GAGE HEIGHT @ St. Lou is: $387.3 \mathrm{ft}$ GAGE HEIGHT @ Cape Girardeau: $319.2 \mathrm{ft}$

RIVER SLOPE: $101 \times 10^{-6}$

SUSP. Bag sampler and 150-lb weight

PRICE AA CURRENT METER No: W-223906 DATE RATED: 06-91

CURRENT METER EQUATION: $V(\mathrm{~m} / \mathrm{s})=\operatorname{Rev} / \mathrm{s}^{*} 0.670+0.010$

REMARKS:

Transit rate was $10 \mathrm{~cm} / \mathrm{s}$ and nozzle size was $1 / 4$ inch. Unmeasured zone was $0.67 \mathrm{~m}$. Order of verticals was $1-4,9-12$, and $8-5$.

\begin{tabular}{|c|c|c|c|c|c|c|c|}
\hline Vertical & $\begin{array}{l}\text { Distance } \\
\text { from } \\
\text { LEW } \\
\text { (m) }\end{array}$ & $\begin{array}{l}\text { Depth } \\
\text { (m) }\end{array}$ & $\begin{array}{c}\text { Mean } \\
\text { velocity } \\
(\mathrm{m} / \mathrm{s})\end{array}$ & $\begin{array}{c}\text { Discharge } \\
\left(\mathrm{m}^{3} / \mathrm{s}\right)\end{array}$ & $\begin{array}{l}\text { Temperature } \\
\left({ }^{\circ} \mathrm{C}\right)\end{array}$ & pH & $\begin{array}{c}\text { Speclfic } \\
\text { conductance } \\
(\mu \mathrm{S} / \mathrm{cm})\end{array}$ \\
\hline LEW & 0 & 0.0 & 0.00 & & & & \\
\hline $01 \mathrm{~A}, \mathrm{~B}$ & 51 & 6.5 & 0.62 & 177 & 8.7 & uncertain & 568 \\
\hline $02 \mathrm{~A}, \mathrm{~B}$ & 88 & 7.3 & 1.09 & 365 & 8.7 & uncertain & 569 \\
\hline $03 \mathrm{~A}, \mathrm{~B}$ & 143 & 8.1 & 1.38 & 492 & 8.6 & 8.2 & 569 \\
\hline 04A,B & 176 & 7.8 & 1.49 & 481 & 8.6 & 8.3 & 570 \\
\hline $05 A, B$ & 226 & 8.1 & 1.37 & 538 & 8.7 & 8.4 & 571 \\
\hline $06 \mathrm{~A}, \mathrm{~B}$ & 273 & 7.1 & 1.20 & 380 & 8.6 & 8.4 & 572 \\
\hline 07A,B & 315 & 5.8 & 1.20 & 307 & 8.7 & 8.4 & 572 \\
\hline 08A,B & 361 & 5.1 & 1.16 & 259 & 8.6 & 8.3 & 571 \\
\hline $09 \mathrm{~A}, \mathrm{~B}$ & 403 & 5.3 & 1.14 & 260 & 8.6 & 8.3 & 570 \\
\hline $10 \mathrm{~A}, \mathrm{~B}$ & 447 & 5.3 & 1.07 & 258 & 8.6 & 8.4 & 568 \\
\hline $11 \mathrm{~A}, \mathrm{~B}$ & 494 & 4.5 & 1.12 & 219 & 8.6 & 8.3 & 567 \\
\hline $12 A, B$ & 542 & 3.8 & 0.75 & 137 & 8.6 & 8.4 & 567 \\
\hline REW & 590 & 0.0 & 0.00 & & & & \\
\hline MEANS & & 5.7 & 1.15 & & & & \\
\hline TOTAL & 590 & & & 3,872 & & & \\
\hline
\end{tabular}




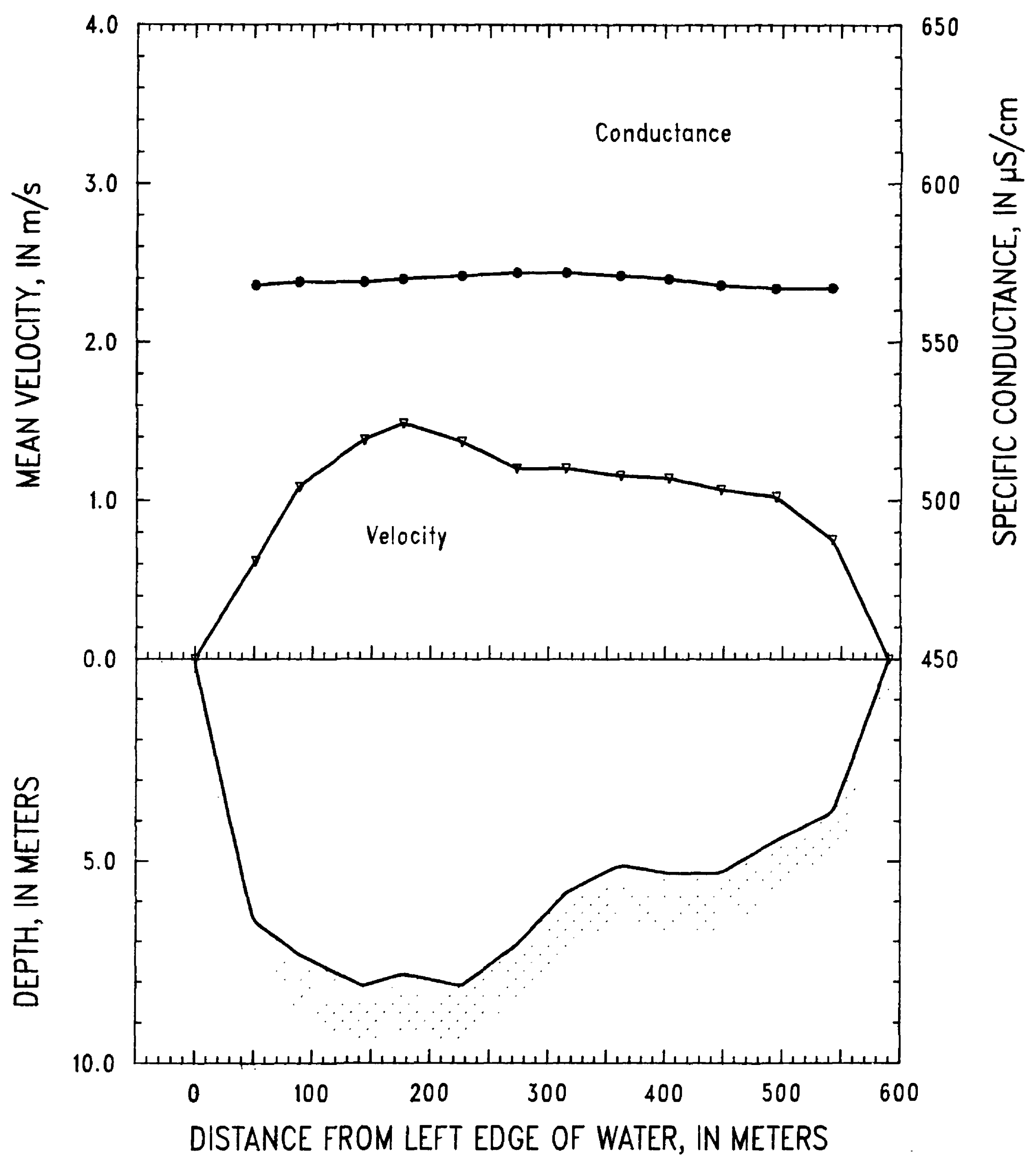

Figure 34. Mississippi River at Thebes, lllinois, on November 5, 1991. 
SITE: Ohio River at Olmsted, Ill.-Mile 965.5

$11-06-91$

PARTY: Moody, Ellis, and Simoneaux

GAGE HEIGHT @ Dam 53: $287.9 \mathrm{ft}$ GAGE HEIGHT @ Cairo: $286.3 \mathrm{ft}$

RIVER SLOPE: $16.5 \times 10^{-6}$

SUSP. Bag sampler and 150-lb weight

PRICE AA CURRENT METER No: W-223906 DATE RATED: 06-91

CURRENT METER EQUATION: $V(\mathrm{~m} / \mathrm{s})=\operatorname{Rev} / \mathrm{s}^{*} 0.670+0.010$

REMARKS:

Did not collect a depth-integrated composite sample. Maximum transit rate was $1.2 \mathrm{~cm} / \mathrm{s}$ and the unmeasured zone was $0.20 \mathrm{~m}$. Specific conductance was measured twice: verticals $11-1$ in the mrning and verticals $1-11$ in the afternoon, shown in parenthesis.

\begin{tabular}{|c|c|c|c|c|c|c|c|}
\hline Vertical & $\begin{array}{l}\text { Distance } \\
\text { from } \\
\text { LEW } \\
\text { (m) }\end{array}$ & $\begin{array}{l}\text { Depth } \\
\text { (m) }\end{array}$ & $\begin{array}{c}\text { Mean } \\
\text { velocity } \\
(\mathrm{m} / \mathrm{s})\end{array}$ & $\begin{array}{c}\text { Discharge } \\
\left(\mathrm{m}^{3} / \mathrm{s}\right)\end{array}$ & $\begin{array}{c}\text { Temperature } \\
\left({ }^{\circ} \mathrm{C}\right)\end{array}$ & pH & $\begin{array}{c}\text { Speclific } \\
\text { conductance } \\
(\mu \mathrm{S} / \mathrm{cm})\end{array}$ \\
\hline LEW & 0 & 0.0 & 0.00 & & & & \\
\hline 01 & 127 & 2.8 & 0.26 & 70 & 13.4 & 7.8 & $287(286)$ \\
\hline 02 & 194 & 3.6 & 0.27 & 71 & 13.4 & 7.8 & $286(285)$ \\
\hline 03 & 274 & 4.8 & 0.33 & 125 & 13.4 & 7.7 & $300(286)$ \\
\hline 04 & 353 & 6.1 & 0.40 & 193 & 13.2 & 7.6 & $289(306)$ \\
\hline 05 & 430 & 7.4 & 0.43 & 236 & 13.3 & 7.6 & $309(302)$ \\
\hline 06 & 502 & 7.6 & 0.54 & 345 & 13.2 & 7.6 & $310(310)$ \\
\hline 07 & 598 & 7.5 & 0.52 & 320 & 13.2 & 7.6 & $297(324)$ \\
\hline 08 & 665 & 7.0 & 0.57 & 295 & 13.2 & 7.6 & 341 (333) \\
\hline 09 & 746 & 7.9 & 0.52 & 331 & 13.2 & 7.6 & $330(373)$ \\
\hline 10 & 825 & 10.0 & 0.38 & 335 & 13.2 & 7.5 & $333(376)$ \\
\hline 11 & 921 & 8.6 & 0.27 & 159 & 13.1 & 7.5 & $353(377)$ \\
\hline REW & 962 & 0.0 & 0.00 & & & & \\
\hline MEANS & & 6.0 & 0.43 & & & & \\
\hline TOTAL & 962 & & & 2,479 & & & \\
\hline
\end{tabular}




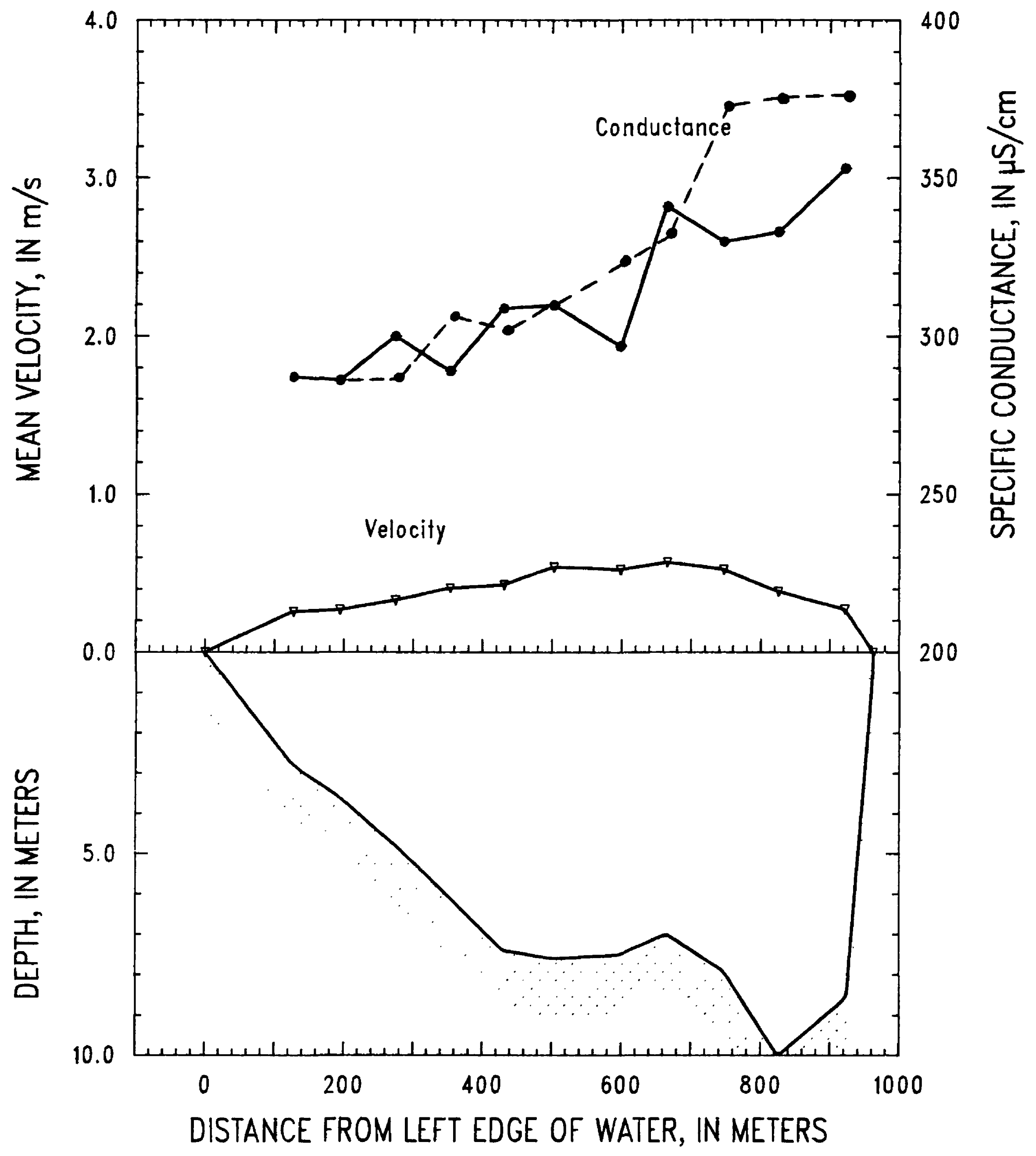

Figure 35. Ohio River at Olmsted, Illinois, on November 6, 1991. 
STTE: Mississippi River below Vicksburg, Miss.-Mile 433.4

11-09-91

PARTY: Moody, Roth, and Simoneaux

GAGE HEIGHT @ Greenville: $94.3 \mathrm{ft}$ GAGE HEIGHT @ Natchez: $36.2 \mathrm{ft}$

RIVER SLOPE: $65.4 \times 10^{-6}$

SUSP. Bag sampler and $150-\mathrm{lb}$ weight

PRICE AA CURRENT METER No: W-223906 DATE RATED: 06-91

CURRENT METER EQUATION: $\mathrm{V}(\mathrm{m} / \mathrm{s})=\operatorname{Rev} / \mathrm{s}^{*} 0.670+0.010$

REMARKS:

Fifteen equally spaced verticals were planned, but only 13 were sampled because of shallow water near the right bank. Transit rate was $9.5 \mathrm{~cm} / \mathrm{s}$ and the nozzle was $3 / 16$ inch. The section was about 12 degrees off orthogonality. Arkansas River water was about $\mathbf{4 0}$ percent of the total discharge. Order of verticals was: $\mathrm{X} 01-\mathrm{X} 04,1-3,9-4$, and 10-13.

\begin{tabular}{|c|c|c|c|c|c|c|c|}
\hline Vertlcal & $\begin{array}{l}\text { Distance } \\
\text { from } \\
\text { LEW } \\
\text { (m) }\end{array}$ & $\begin{array}{l}\text { Depth } \\
\text { (m) }\end{array}$ & $\begin{array}{l}\text { Mean } \\
\text { velocity } \\
(\mathrm{m} / \mathrm{s})\end{array}$ & $\begin{array}{c}\text { Dlscharge } \\
\left(\mathrm{m}^{3} / \mathrm{s}\right)\end{array}$ & $\begin{array}{c}\text { Temperature } \\
\left({ }^{\circ} \mathbf{C}\right)\end{array}$ & pH & $\begin{array}{c}\text { Speclfic } \\
\text { conductance } \\
(\mu \mathrm{S} / \mathrm{cm})\end{array}$ \\
\hline LEW & 0 & 0.0 & & & & & \\
\hline 01B & 78 & 9.0 & 0.85 & 317 & 11.4 & 7.3 & 381 \\
\hline $01 \mathrm{~A}$ & 83 & 13.1 & 0.96 & 214 & 11.4 & 7.1 & 381 \\
\hline$X 04$ & 112 & 15.3 & 1.07 & 644 & - & - & -- \\
\hline 02A,B & 162 & 14.5 & 1.24 & 878 & 11.4 & 7.6 & 399 \\
\hline $\mathrm{X} 03$ & 210 & 13.7 & 1.25 & 523 & -. & -- & - \\
\hline $03 A, B$ & 223 & 13.8 & 1.21 & 777 & 11.4 & 7.7 & 403 \\
\hline $04 A, B$ & 303 & 13.4 & 1.25 & 1,279 & 11.5 & 7.9 & 424 \\
\hline 05A,B & 376 & 13.7 & 1.10 & 1,149 & 11.4 & 7.9 & 431 \\
\hline 06A,B & 456 & 12.4 & 1.06 & 881 & 11.5 & 7.9 & 432 \\
\hline $\mathrm{X} 02$ & 510 & 12.0 & 1.04 & 398 & - & -- & -- \\
\hline 07A,B & 520 & 12.3 & 1.05 & 562 & 11.5 & 7.9 & 435 \\
\hline 08A,B & 597 & 12.5 & 0.97 & 907 & 11.5 & 7.8 & 433 \\
\hline $09 \mathrm{~A}, \mathrm{~B}$ & 670 & 11.5 & 0.88 & 739 & 11.5 & 7.8 & 433 \\
\hline $10 \mathrm{~A}, \mathrm{~B}$ & 743 & 9.3 & 0.84 & 536 & 11.5 & 7.9 & 433 \\
\hline $11 \mathrm{~A}, \mathrm{~B}$ & 807 & 7.2 & 0.79 & 400 & 12.3 & 7.9 & 438 \\
\hline $12 A, B$ & 883 & 5.1 & 0.58 & 183 & 11.8 & 7.9 & 435 \\
\hline$x 01$ & 931 & 4.0 & 0.49 & 69 & -- & - & -- \\
\hline $13 A, B$ & 954 & 3.7 & 0.51 & 235 & 11.5 & 8.0 & 435 \\
\hline REW & 1,180 & 0.0 & & & & & \\
\hline MEANS & & 9.0 & 1.01 & & & & \\
\hline TOTAL & 1,180 & & & 10,689 & & & \\
\hline
\end{tabular}




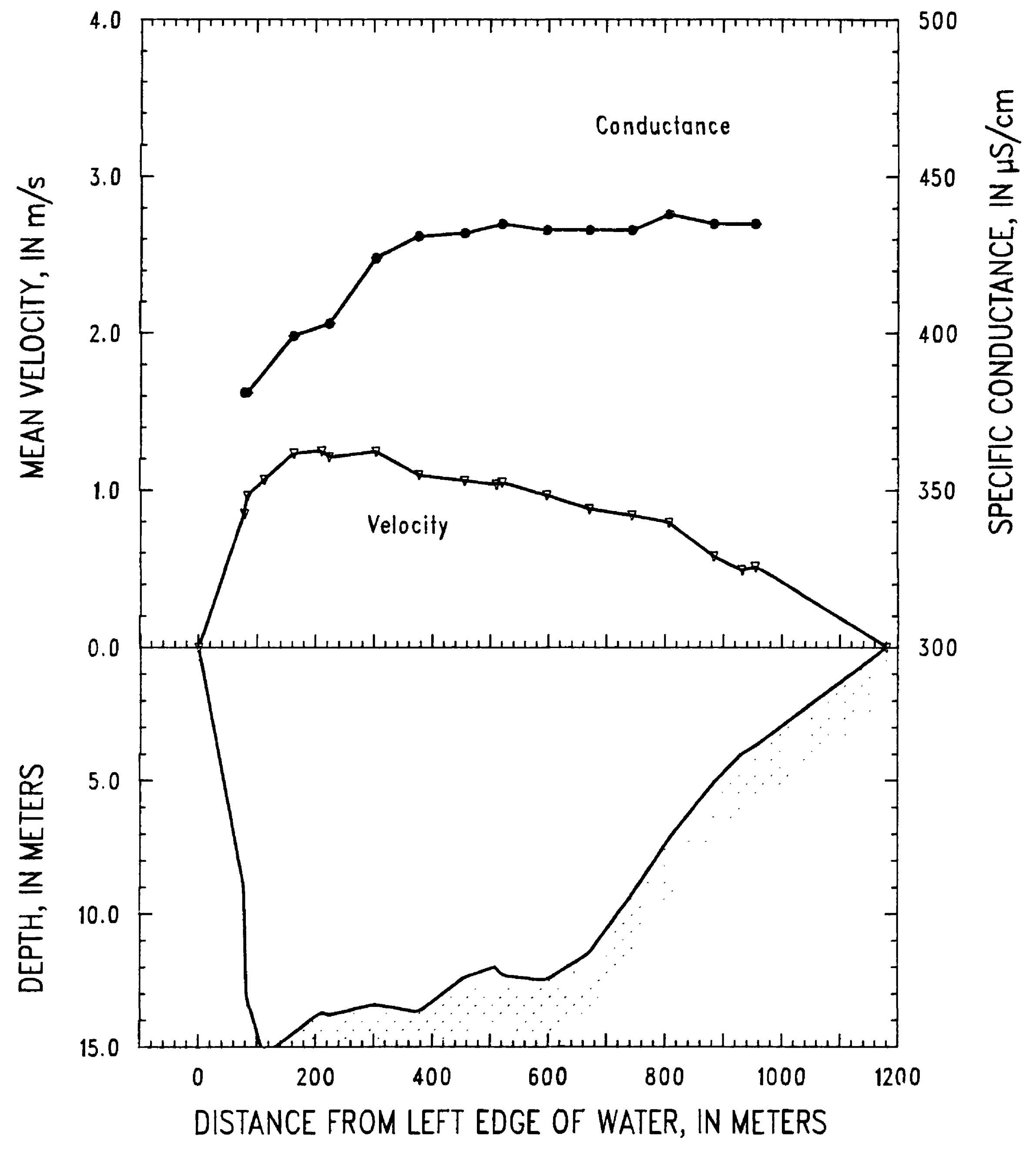

Figure 36. Mississippi River below Vicksburg, Mississippi, on November 9, 1991. 
SITE: Mississippi River near St. Francisville, La.-Mile 266.4

$11-11-91$

PARTY: Moody, Roth, Simoneaux, and LeBoeuf

GAGE HEIGHT @ Red River Landing: $25.5 \mathrm{ft} \quad$ GAGE HEIGHT @ Baton Rouge: $12.4 \mathrm{ft}$

RIVER SLOPE: $33.5 \times 10^{-6}$

SUSP. Bag sampler and 150-lb weight

PRICE AA CURRENT METER No: W-223906 DATE RATED: 06-91

CURRENT METER EQUATION: $V(\mathrm{~m} / \mathrm{s})=\operatorname{Rev} / \mathrm{s}^{*} 0.670+0.010$

REMARKS:

Transit rate was $7.5 \mathrm{~cm} / \mathrm{s}$ and the nozzle was 3/16 inch. Gage heights and river slope are for November 9,1991 . Order of verticals $w$ as $12-9,5,4,8,3,2,1,6$, and 7 .

\begin{tabular}{|c|c|c|c|c|c|c|c|}
\hline Vertical & $\begin{array}{l}\text { Distance } \\
\text { from } \\
\text { LEW } \\
\text { (m) }\end{array}$ & $\begin{array}{l}\text { Depth } \\
\text { (m) }\end{array}$ & $\begin{array}{l}\text { Mean } \\
\text { velocity } \\
(\mathbf{m} / \mathbf{s})\end{array}$ & $\begin{array}{c}\text { Discharge } \\
\left(\mathrm{m}^{3} / \mathrm{s}\right)\end{array}$ & $\begin{array}{l}\text { Temperature } \\
\left({ }^{\circ} \mathrm{C}\right)\end{array}$ & pH & $\begin{array}{c}\text { Specific } \\
\text { conductance } \\
(\mu \mathrm{S} / \mathrm{cm})\end{array}$ \\
\hline LEW & 0 & 0.0 & 0.00 & & & & \\
\hline $01 \mathrm{~A}, \mathrm{~B}$ & 80 & 5.5 & 0.73 & 311 & 11.7 & 7.8 & 430 \\
\hline $02 \mathrm{~A}, \mathrm{~B}$ & 154 & 7.5 & 0.77 & 439 & 11.8 & 7.8 & 430 \\
\hline 03A,B & 232 & 7.1 & 0.74 & 391 & 11.6 & 7.8 & 431 \\
\hline $04 \mathrm{~A}, \mathrm{~B}$ & 303 & 7.2 & 0.78 & 229 & 11.6 & 7.8 & 432 \\
\hline $\mathrm{X03}$ & 314 & 7.9 & 0.83 & 248 & - & -- & -- \\
\hline $05 A, B$ & 379 & 8.1 & 0.79 & 430 & 11.7 & 7.8 & 430 \\
\hline $06 \mathrm{~A}, \mathrm{~B}$ & 448 & 9.7 & 0.83 & 607 & 11.7 & 7.8 & 430 \\
\hline $07 A, B$ & 529 & 10.6 & 1.05 & 906 & 11.9 & 7.8 & 430 \\
\hline $\mathrm{X} 02$ & 611 & 12.5 & 1.08 & 610 & -- & -- & - \\
\hline $08 \mathrm{~A}, \mathrm{~B}$ & 619 & 12.1 & 1.08 & 420 & 11.6 & 7.8 & 430 \\
\hline $09 \mathrm{~A}, \mathrm{~B}$ & 675 & 11.9 & 1.03 & 847 & 11.5 & 7.8 & 430 \\
\hline $10 \mathrm{~A}, \mathrm{~B}$ & 757 & 14.2 & 1.07 & 936 & 11.6 & 7.7 & 430 \\
\hline X01 & 798 & 16.3 & 1.04 & 605 & -- & -- & - \\
\hline $11 \mathrm{~A}, \mathrm{~B}$ & 828 & 14.2 & 1.13 & 828 & 11.4 & 7.6 & 430 \\
\hline $12 \mathrm{~A}, \mathrm{~B}$ & 901 & 13.3 & 1.18 & 1,145 & 11.5 & 7.3 & 431 \\
\hline REW & 974 & 0.0 & 0.00 & & & & \\
\hline MEANS & & 9.4 & 0.98 & & & & \\
\hline TOTAL & 974 & & & 8,950 & & & \\
\hline
\end{tabular}




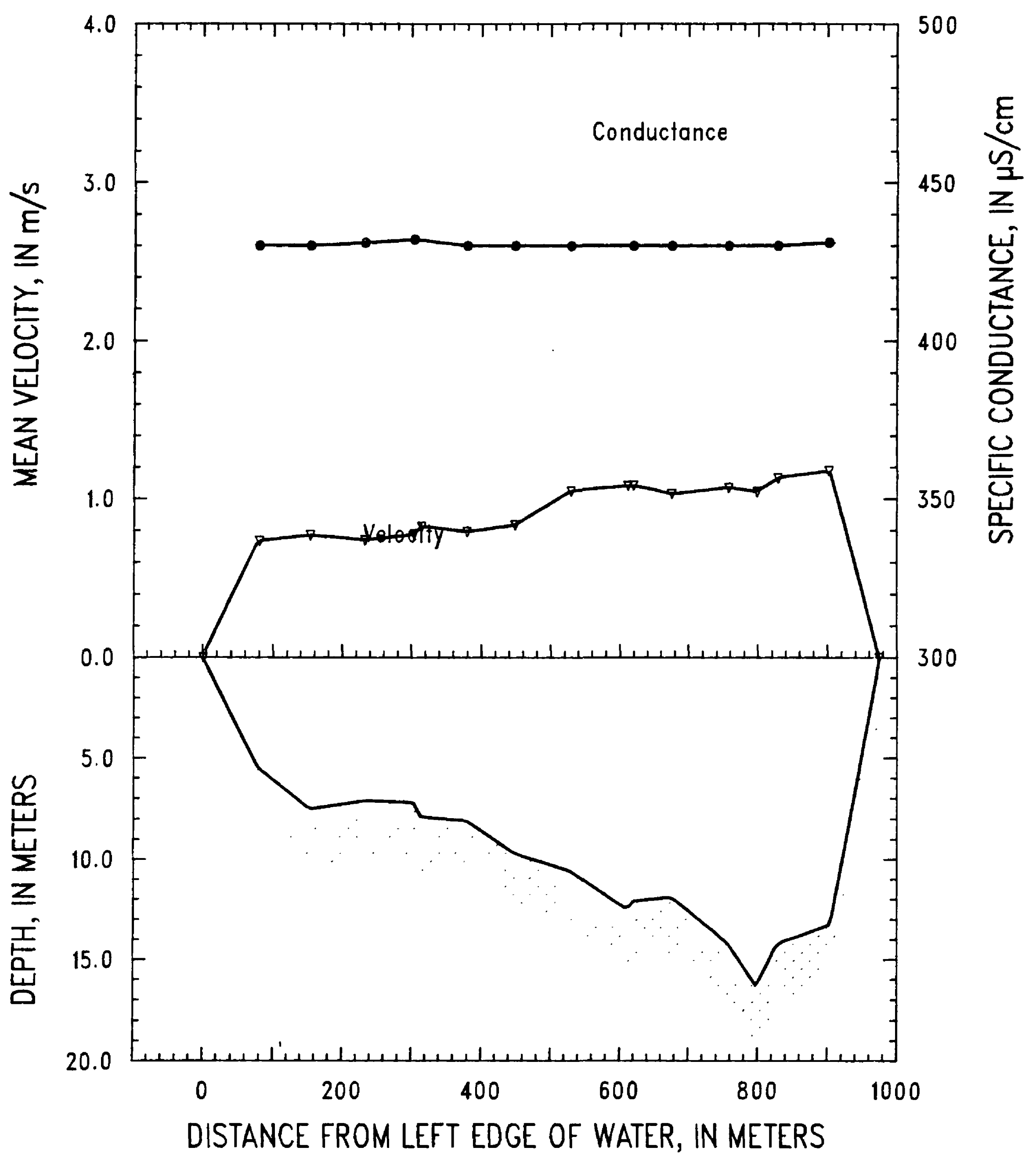

Figure 37. Mississippi River near St. Francisville, Louisiana, on November 11, 1991. 
SITE: Mississippi River below Belle Chasse, La.-Mile 73.1

PARTY: Moody, Roth, Ellis, and Simoneaux

GAGE HEIGHT @ Baton Rouge: $12.7 \mathrm{ft}$ GAGE HEIGHT @ New Orleans: $3.3 \mathrm{ft}$

RIVER SLOPE: $14.2 \times 10^{-6}$

SUSP. Bag sampler and 150-lb weight

PRICE AA CURRENT METER No: W-223906 DATE RATED: 06-91

CURRENT METER EQUATION: $V(\mathrm{~m} / \mathrm{s})=\operatorname{Rev} / \mathrm{s}^{*} 0.670+0.010$

REMARKS:

Transit rate was $6.0 \mathrm{~cm} / \mathrm{s}$ and the nozzle was 1/4 inch. Gage heights and slope are for Novermber $11,1991$.

Order of verticals was $5-1,6$, and 7 .

\begin{tabular}{lccccccc}
\hline Verticai & $\begin{array}{c}\text { Distance } \\
\text { from } \\
\text { LEW } \\
(\mathbf{m})\end{array}$ & $\begin{array}{c}\text { Depth } \\
(\mathrm{m})\end{array}$ & $\begin{array}{c}\text { Mean } \\
\text { velocity } \\
(\mathbf{m} / \mathbf{s})\end{array}$ & $\begin{array}{c}\text { Discharge } \\
\left(\mathbf{m}^{3} / \mathbf{s}\right)\end{array}$ & $\begin{array}{c}\text { Temperature } \\
\left({ }^{\circ} \mathbf{C}\right)\end{array}$ & pH & $\begin{array}{c}\text { Speclfic } \\
\text { conductance } \\
(\mu \mathrm{S} / \mathbf{c m})\end{array}$ \\
\hline LEW & 0 & 0.0 & 0.00 & & & & \\
06A & 66 & 14.0 & 0.41 & 400 & 14.5 & 7.4 & 489 \\
01A & 140 & 21.0 & 0.57 & 1,396 & 12.8 & 7.8 & 490 \\
02A & 299 & 23.0 & 0.69 & 2,334 & 13.9 & 7.8 & 488 \\
03A & 433 & 24.6 & 0.63 & 2,011 & 14.1 & 7.8 & 488 \\
04A & 560 & 24.2 & 0.49 & 1.319 & 14.0 & 7.8 & 488 \\
05A & 655 & 22.8 & 0.46 & 794 & 14.1 & 7.6 & 492 \\
07A & 712 & 21.9 & 0.42 & 585 & 14.2 & 7.4 & 488 \\
REW & 781 & 0.0 & 0.00 & & & & \\
MEANS & & 20.2 & 0.56 & & & & \\
TOTAL & 781 & & & 8,838 & & & \\
\hline
\end{tabular}




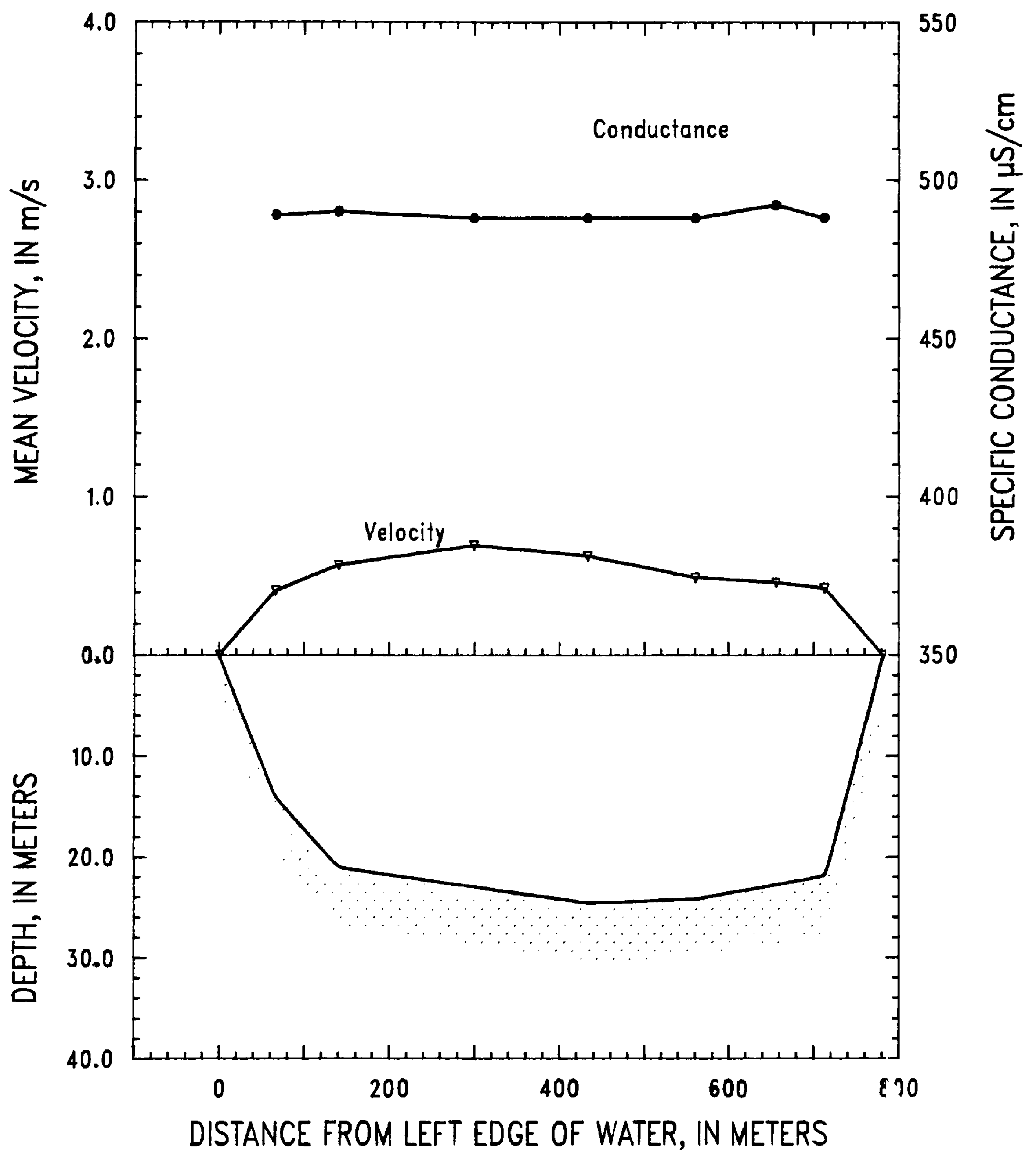

Figure 38. Mississippi River below Belle Chasse, Louisiana, on November 13, 1991. 


$$
\text { wo }
$$




\title{
DATA LISTINGS
}

\author{
FOR
}

APRIL-MAY 1992 CRUISE 
SITE: Mississippi River above St. Anthony Falls, Minn.-Mile 857.7

PARTY: Moody, Roth, and LeBoeuf

GAGE HEIGHT @ Anoka: 809.13 ft GAGE HEIGHT @ SAFU Pool: $799.56 \mathrm{ft}$

RIVER SLOPE: $168 \times 10^{-6}$

SUSP. Bag sampler and 150 -lb weight

PRICE AA CURRENT METER No: W-297222 DATE RATED: 06-91

CURRENT METER EQUATION: $\mathrm{V}(\mathrm{m} / \mathrm{s})=\operatorname{Rev} / \mathrm{s}^{*} 0.670+0.010$

REMARKS:

Anchored at four verticals and free-boated at vertical 5 . Variable transit rate $(1-4 \mathrm{~cm} / \mathrm{s})$ and $5 / 16-\mathrm{inch}$ nozzle. Unmeasured zone was 0.7 and $0.3 \mathrm{~m}$ (at $X$-labeled verticals). River was unmixed at mile 859.6. O-der of verticals was $3,1,2,4$, and 5 . Temperature, $\mathrm{pH}$, and specific conductance are the mean of three measurements.

\begin{tabular}{|c|c|c|c|c|c|c|c|}
\hline Vertical & $\begin{array}{l}\text { Dlstance } \\
\text { from } \\
\text { LEW } \\
\text { (m) }\end{array}$ & $\begin{array}{l}\text { Depth } \\
\text { (m) }\end{array}$ & $\begin{array}{c}\text { Mean } \\
\text { velocity } \\
(\mathrm{m} / \mathrm{s})\end{array}$ & $\begin{array}{c}\text { Discharge } \\
\left(\mathrm{m}^{3} / \mathrm{s}\right)\end{array}$ & $\begin{array}{c}\text { Temperature } \\
\left({ }^{\circ} \mathrm{C}\right)\end{array}$ & pH & $\begin{array}{c}\text { Speciflc } \\
\text { conductance } \\
(\mu \mathrm{S} / \mathrm{cm})\end{array}$ \\
\hline LEW & 0 & 0.0 & 0.00 & & & & \\
\hline$x 01$ & 26 & 2.7 & 0.75 & 40 & - & -- & -- \\
\hline $01 \mathrm{~A}, \mathrm{~B}$ & 39 & 3.7 & 0.72 & 44 & 10.6 & 8.7 & 365 \\
\hline $\mathrm{X} 02$ & 59 & 3.8 & 0.64 & 29 & -- & -- & - \\
\hline $02 \mathrm{~A}, \mathrm{~B}$ & 63 & 3.7 & 0.61 & 25 & 10.6 & 8.7 & 381 \\
\hline 03A,B & 81 & 2.9 & 0.63 & 24 & 10.3 & 8.7 & 392 \\
\hline$\times 03$ & 89 & 3.6 & 0.76 & 56 & -- & -- & -- \\
\hline$X 04$ & 122 & 4.8 & 0.57 & 52 & -- & -- & -- \\
\hline $04 \mathrm{~A}, \mathrm{~B}$ & 127 & 4.7 & 0.53 & 28 & 10.6 & 8.7 & 446 \\
\hline $05 \mathrm{~A}, \mathrm{~B}$ & 144 & 3.0 & 0.28 & 8 & 11.1 & 8.7 & 482 \\
\hline$\times 05$ & 146 & 2.7 & 0.19 & 6 & & & \\
\hline REW & 168 & 0.0 & 0.00 & & & & \\
\hline MEANS & & 3.1 & 0.60 & & & & \\
\hline TOTAL & 168 & & & 310 & & & \\
\hline
\end{tabular}




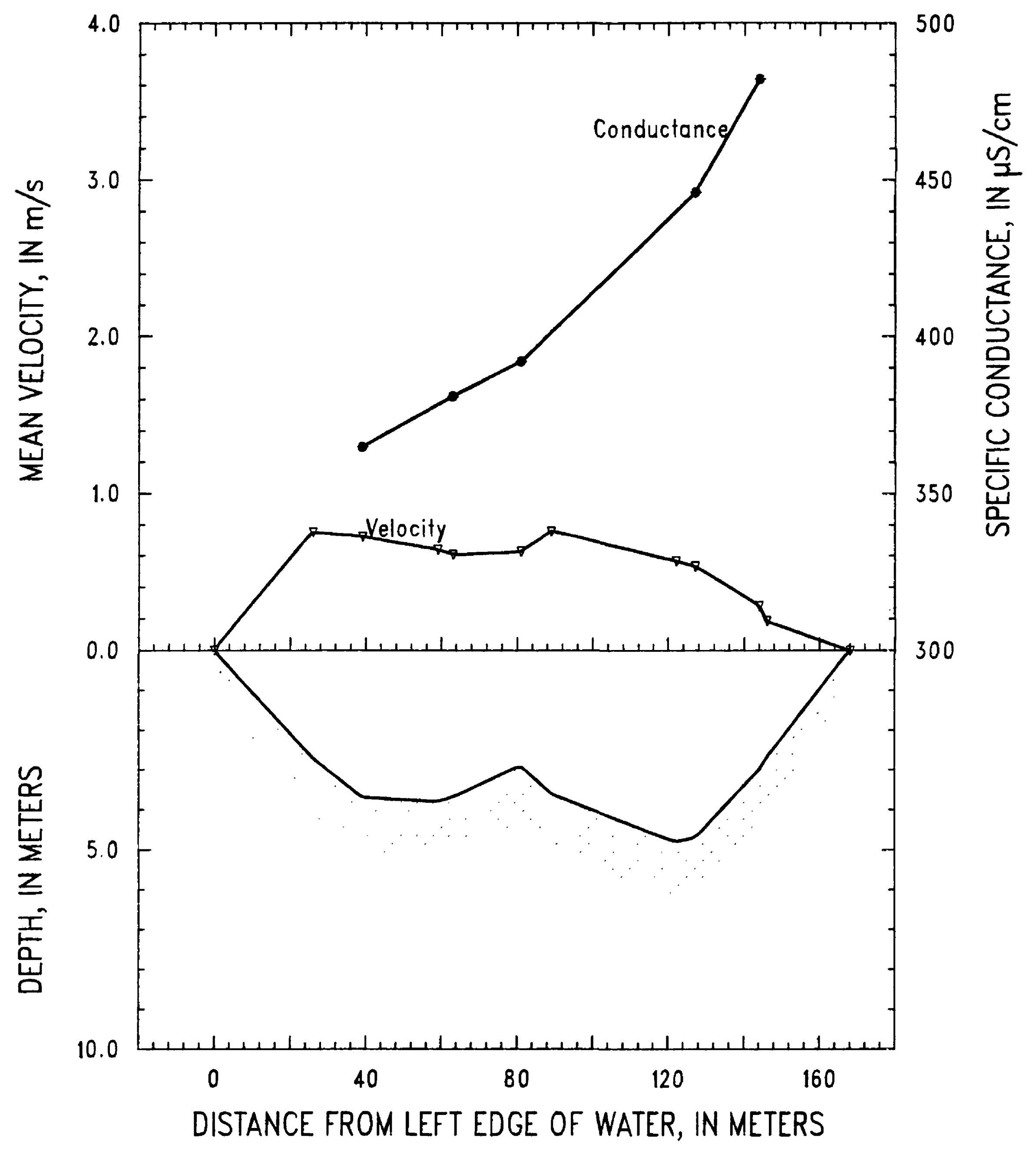

Figure 39. Mississippi River above St. Anthony Falls, Minnesota, on April 6, 1992. 
SITE: Mississippi River at Mile 844.0, Minn.

04-07-92

PARTY: Moody and Garbarino

GAGE HEIGHT @ Savage: 692.77 ft GAGE HEIGHT @ Pool 2: $686.50 \mathrm{ft}$

RIVER SLOPE: $27 \times 10^{-6}$

SUSP. 15-lb weight

PRICE AA CURRENT METER No: 90JM-1 DATE RATED: 06-91

CURRENT METER EQUATION: $V(\mathrm{~m} / \mathrm{s})=\mathrm{Rev} / \mathrm{s}^{*} 0.667+0.006$

REMARKS:

Anchored at five verticals using the small boat. Depth integrated measurement by hand. Specific conductance and temperature were measured by a Sea Bird, SeaCat profiler.

\begin{tabular}{|c|c|c|c|c|c|c|c|}
\hline Verticai & $\begin{array}{l}\text { Distance } \\
\text { from } \\
\text { LEW } \\
\text { (m) }\end{array}$ & $\begin{array}{l}\text { Depth } \\
\text { (m) }\end{array}$ & $\begin{array}{l}\text { Mean } \\
\text { velocity } \\
(\mathbf{m} / \mathbf{s})\end{array}$ & $\begin{array}{c}\text { Discharge } \\
\left(\mathrm{m}^{3} / \mathrm{s}\right)\end{array}$ & $\begin{array}{c}\text { Temperature } \\
\left({ }^{\circ} \mathrm{C}\right)\end{array}$ & pH & $\begin{array}{c}\text { Specific } \\
\text { conductance } \\
(\mu \mathrm{S} / \mathrm{cm})\end{array}$ \\
\hline LEW & 0 & 0.0 & 0.00 & & & & \\
\hline 01 & 22 & 5.8 & 0.55 & 71 & 9.6 & -- & 421 \\
\hline 02 & 44 & 6.4 & 0.61 & 74 & 9.6 & -- & 424 \\
\hline 03 & 60 & 6.7 & 0.62 & 73 & 9.6 & -- & 431 \\
\hline 04 & 79 & 6.3 & 0.56 & 84 & 9.6 & -- & 440 \\
\hline 05 & 108 & 5.7 & 0.29 & 55 & 9.6 & -- & 466 \\
\hline REW & 145 & 0.0 & 0.00 & & & & \\
\hline MEANS & & 4.9 & 0.51 & & & & \\
\hline TOTAL & 145 & & & 357 & & & \\
\hline
\end{tabular}




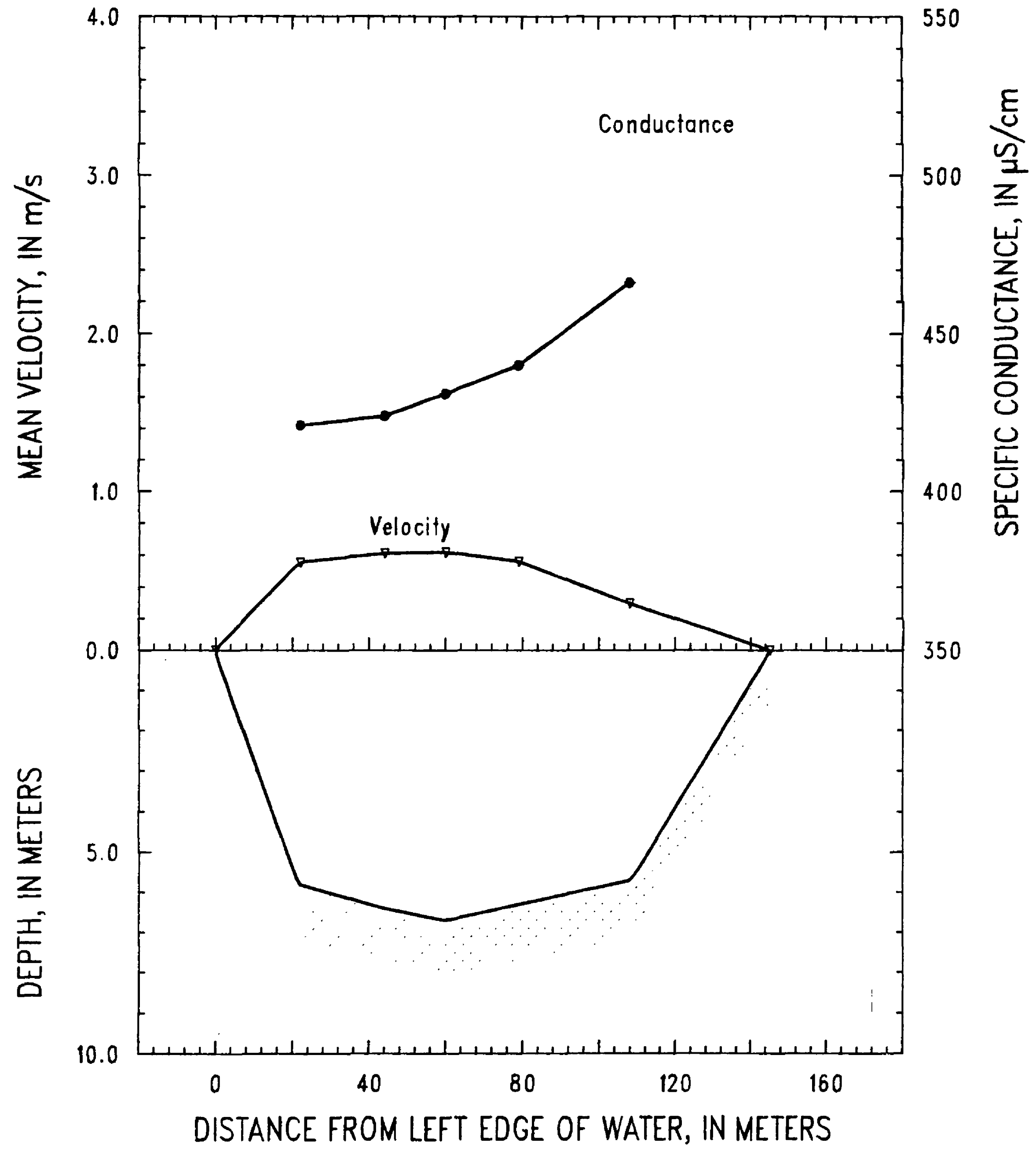

Figure 40. Mississippi River at Mile 844.0, Minnesota, on April 7, 1992. 
SITE: Minnesota River at Mile 3.5, Minn.

04-08-92

PARTY: Moody, Roth, and LeBoeuf

GAGE HEIGHT @ Savage: 692.50 ft GAGE HEIGHT @ Pool 2: $686.58 \mathrm{ft}$

RIVER SLOPE: $25 \times 10^{-6}$

SUSP. Bag sampler and 150 -lb weight

PRICE AA CURRENT METER No: W-223906 DATE RATED: 06-91

CURRENT METER EQUATION: $V(\mathrm{~m} / \mathrm{s})=\operatorname{Rev} / \mathrm{s}^{*} 0.670+0.010$

REMARKS:

Discharge based on velocity and depth measurements from an anchored boat at four verticals. Used

5/16-inch nozzle and a variable transit rate. Microwave remote units were $9 \mathrm{~m}$ from the LEW and the REW.

Unmeasured zone was $0.66 \mathrm{~m}$. Order of verticals was $2,1,3$, and 4 . Temperature, pH, and specific conductance are the mean of three measurements.

\begin{tabular}{lccccccc}
\hline Verticai & $\begin{array}{c}\text { Distance } \\
\text { from } \\
\text { LEW } \\
(\mathrm{m})\end{array}$ & $\begin{array}{c}\text { Depth } \\
(\mathrm{m})\end{array}$ & $\begin{array}{c}\text { Mean } \\
\text { veiocity } \\
(\mathrm{m} / \mathrm{s})\end{array}$ & $\begin{array}{c}\text { Dlscharge } \\
\left(\mathrm{m}^{3} / \mathrm{s}\right)\end{array}$ & $\begin{array}{c}\text { Temperature } \\
\left({ }^{\circ} \mathrm{C}\right)\end{array}$ & pH & $\begin{array}{c}\text { Specific } \\
\text { conductance } \\
(\mu \mathrm{S} / \mathrm{cm})\end{array}$ \\
\hline LEW & 0 & 0.0 & 0.00 & & & & \\
01 & 24 & 4.9 & 0.60 & 70 & 10.1 & 8.4 & 863 \\
02 & 48 & 6.1 & 0.64 & 76 & 9.7 & 8.4 & 862 \\
03 & 63 & 6.0 & 0.67 & 78 & 10.5 & 8.4 & 866 \\
04 & 87 & 4.5 & 0.51 & 35 & 11.0 & 8.4 & 868 \\
REW & 93 & 0.0 & 0.00 & & & & \\
MEANS & & 4.5 & 0.62 & & & & \\
TOTAL & 93 & & & 259 & & & \\
\hline
\end{tabular}




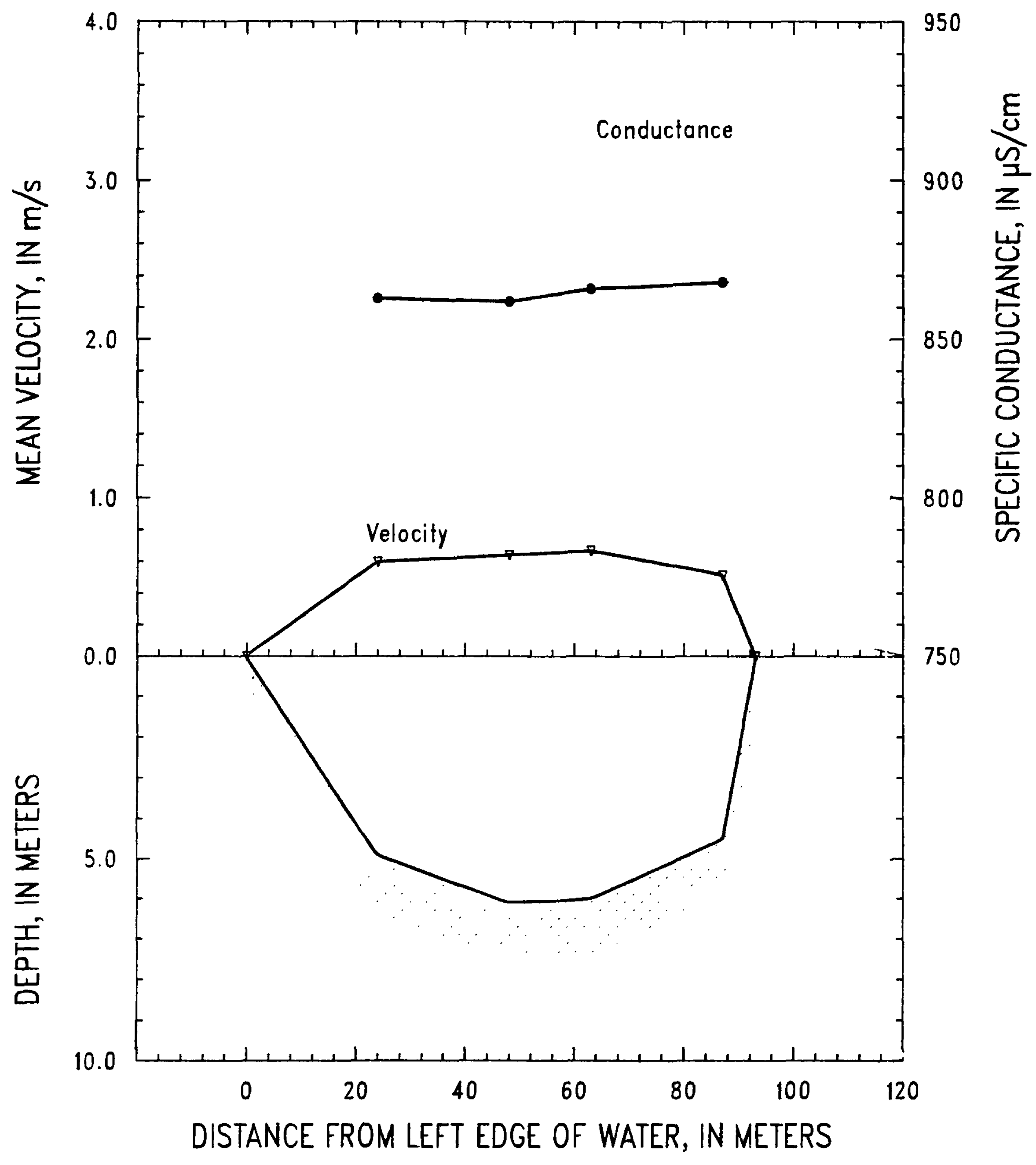

Figure 41. Minnesota River at Mile 3.5, Minnesota, on April 8, 1992. 
SITE: Minnesota River at Mile 0.0, Minn.

PARTY: Moody and Garbarino

GAGE HEIGHT @ Savage: 692.77 ft GAGE HEIGHT @ Pool 2: $686.50 \mathrm{ft}$

RIVER SLOPE: $27 \times 10^{-6}$

SUSP. 15-lb weight

PRICE AA CURRENT METER No: 90JM-1 DATE RATED: 06-91

CURRENT METER EQUATION: $V(\mathrm{~m} / \mathrm{s})=\operatorname{Rev} / \mathrm{s}^{*} 0.667+0.006$

REMARKS:

Anchored at four verticals using the small boat. Depth integrated measurement by hand. Verticals 1-5 were in the Mississippi River. Unmeasured zone was $0.17 \mathrm{~m}$. Temperature and specific conductance were measured by a Sea Bird, SeaCat profiler.

\begin{tabular}{lccccccc}
\hline Vertical & $\begin{array}{c}\text { Distance } \\
\text { from } \\
\text { LEW } \\
(\mathbf{m})\end{array}$ & $\begin{array}{c}\text { Depth } \\
(\mathbf{m})\end{array}$ & $\begin{array}{c}\text { Mean } \\
\text { veiocity } \\
(\mathbf{m} / \mathbf{s})\end{array}$ & $\begin{array}{c}\text { Discharge } \\
\left(\mathrm{m}^{3} / \mathbf{s}\right)\end{array}$ & $\begin{array}{c}\text { Temperature } \\
\left({ }^{\circ} \mathrm{C}\right)\end{array}$ & pH & $\begin{array}{c}\text { Spocific } \\
\text { conductance } \\
(\mu \mathrm{S} / \mathrm{cm})\end{array}$ \\
\hline LEW & 0 & 0.0 & & & & & \\
09 & 41 & 4.0 & 0.71 & 83 & 9.0 & - & 896 \\
08 & 59 & 4.8 & 0.88 & 78 & 8.9 & -- & 896 \\
07 & 78 & 5.0 & 0.80 & 88 & 8.9 & -- & 897 \\
06 & 103 & 4.7 & 0.67 & 63 & 8.8 & -- & 898 \\
REW & 141 & 0.0 & & & & & \\
MEANS & & 2.9 & 0.75 & & & & \\
TOTAL & 141 & & & 312 & & & \\
\hline
\end{tabular}




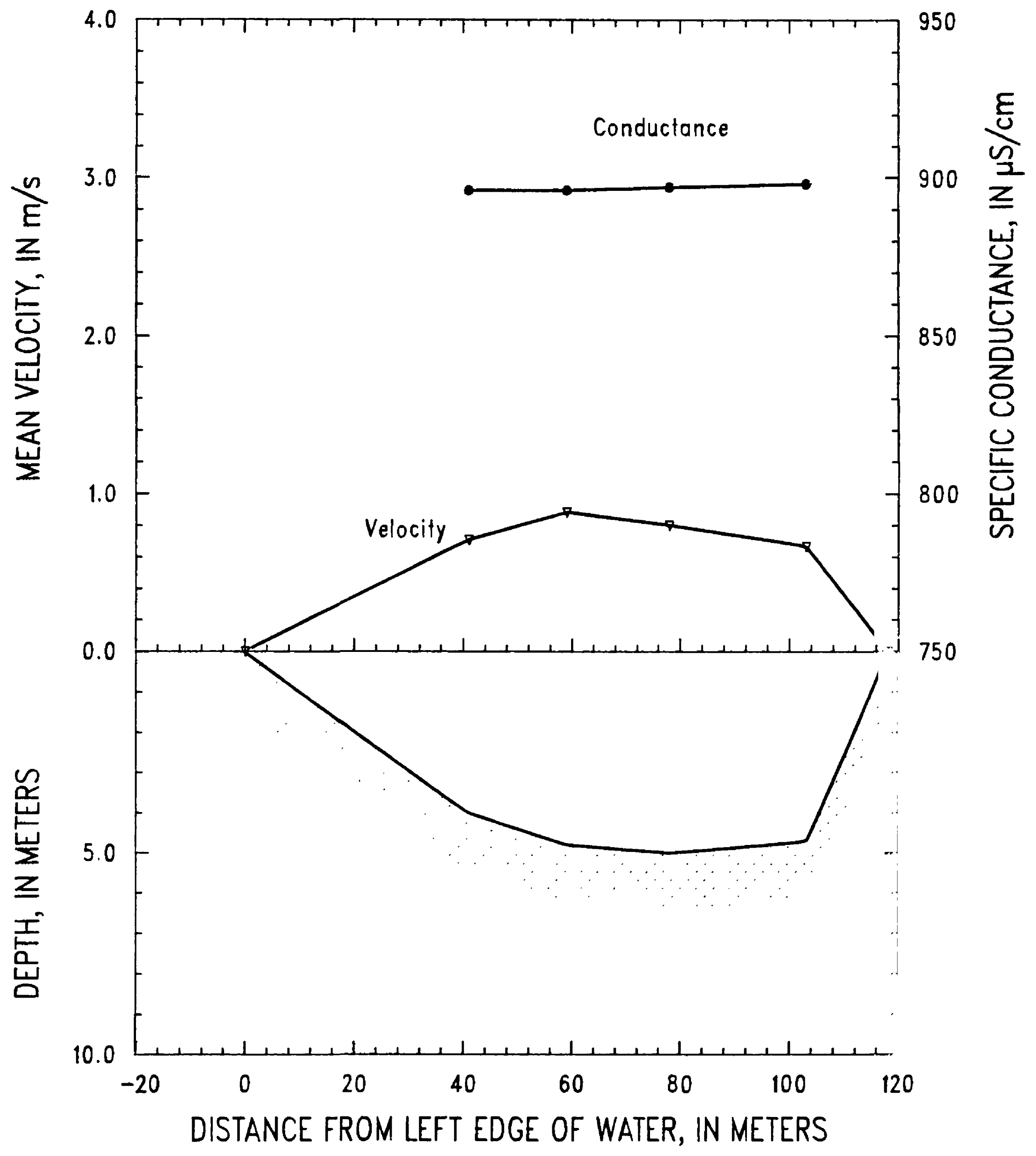

Figure 42. Minnesota River at Mile 0.0, Minnesota, on April 7, $19 \subseteq 2$. 
SITE: Mississippi River at Hastings, Minn.-Mile 812.2

PARTY: Moody, Roth, and LeBoeuf

GAGE HEIGHT @ TW Pool 2: 678.05 ft GAGE HEIGHT @ Pool 3: 674.24 ft

RIVER SLOPE: $40 \times 10^{-6}$

SUSP. Bag sampler and 150 -lb weight

PRICE AA CURRENT METER No: W-223906 DATE RATED: 06-91

CURRENT METER EQUATION: $\mathrm{V}(\mathrm{m} / \mathrm{s})=\mathrm{Rev} / \mathrm{s} * 0.670+0.010$

REMARKS:

Gusty, cold wind with rain, sleet, and snow that blew upriver. Lost the 300-pound pump we'ght. Used the 5/16-inch nozzle and various transit rates. Temperature, $\mathrm{pH}$, and specific conductance are the $\mathrm{m}$ ?an of three measurements.

\begin{tabular}{|c|c|c|c|c|c|c|c|}
\hline Vertical & $\begin{array}{l}\text { Distence } \\
\text { from } \\
\text { LEW } \\
\text { (m) }\end{array}$ & $\begin{array}{l}\text { Depth } \\
\text { (m) }\end{array}$ & $\begin{array}{l}\text { Moen } \\
\text { velocity } \\
(\mathrm{m} / \mathrm{s})\end{array}$ & $\begin{array}{c}\text { Dlscherge } \\
\left(\mathrm{m}^{3} / \mathrm{s}\right)\end{array}$ & $\begin{array}{c}\text { Temperature } \\
\left({ }^{\circ} \mathrm{C}\right)\end{array}$ & pH & $\begin{array}{c}\text { Specific } \\
\text { conductance } \\
(\mu \mathrm{S} / \mathrm{cm})\end{array}$ \\
\hline LEW & 0 & 0.0 & 0.00 & & & & \\
\hline $01 \mathrm{~A}$ & 26 & 8.3 & 0.53 & 102 & 9.8 & 8.6 & 634 \\
\hline $02 \mathrm{~A}$ & 46 & 7.5 & 0.59 & 114 & 9.5 & 8.6 & 633 \\
\hline 03A & 77 & 6.7 & 0.52 & 67 & 9.2 & 8.6 & 633 \\
\hline$X 03$ & 85 & 6.5 & 0.47 & 45 & - & - & - \\
\hline $04 \mathrm{~A}$ & 107 & 5.7 & 0.57 & 73 & 9.0 & 8.6 & 633 \\
\hline 05A & 130 & 5.4 & 0.51 & 69 & 8.8 & 8.6 & 632 \\
\hline $06 \mathrm{~A}$ & 157 & 4.7 & 0.42 & 51 & 8.7 & 8.6 & 631 \\
\hline 07A & 182 & 3.8 & 0.32 & 19 & 8.8 & 8.6 & 632 \\
\hline$\times 07$ & 189 & 3.7 & 0.33 & 26 & - & -. & - \\
\hline REW & 225 & 0.0 & 0.00 & & & & \\
\hline MEANS & & 5.0 & 0.50 & & & & \\
\hline TOTAL & 225 & & & 567 & & & \\
\hline
\end{tabular}




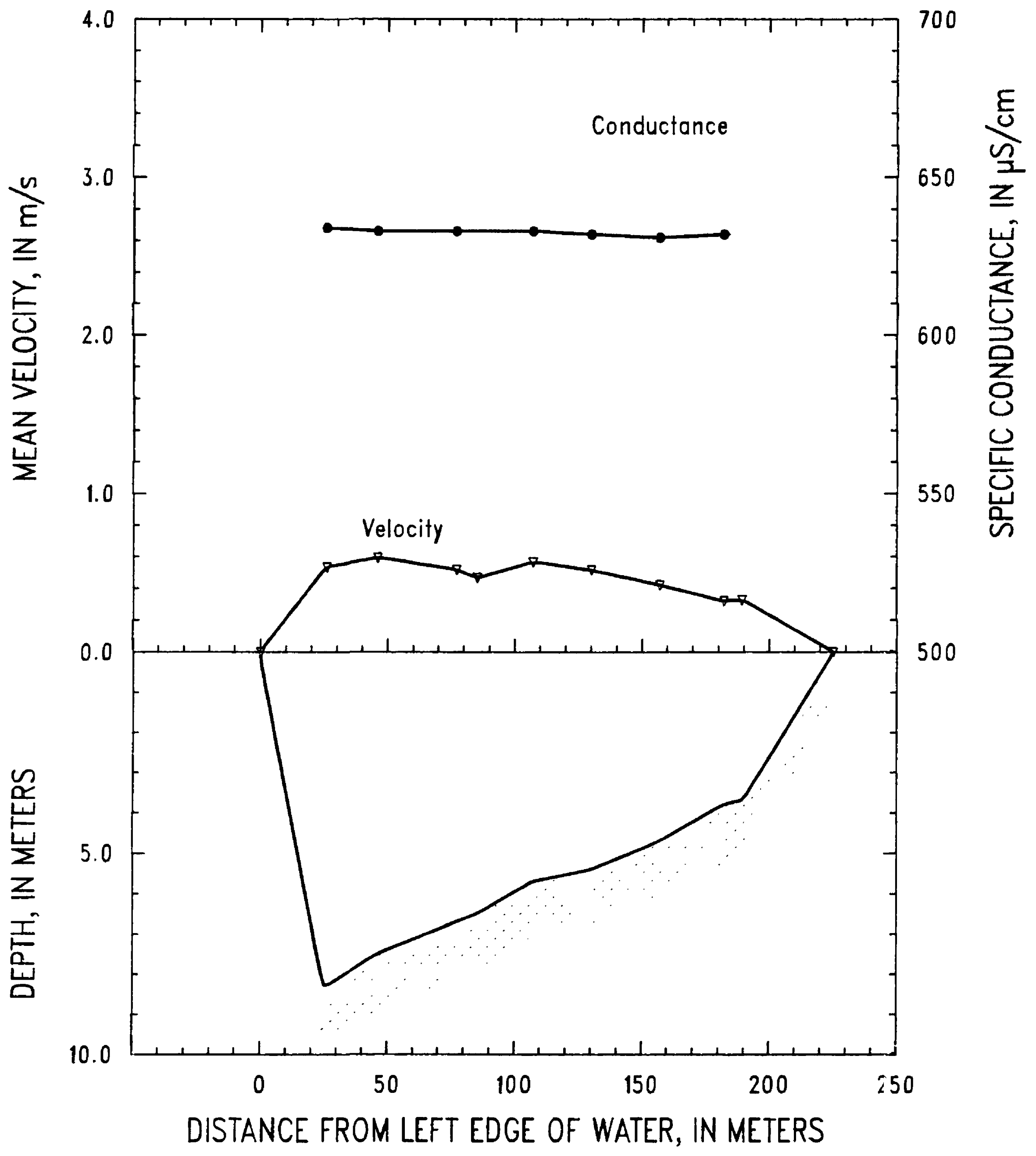

Figure 43. Mississippi River at Hastings, Minnesota, on April 10, 1992. 
SITE: St. Croix River at Mile 0.0, Minn.-Wis.

PARTY: Moody and Garbarino

GAGE HEIGHT @ Dam 2 TW: 678.09ft GAGE HEIGHT @ Pool 3: $674.26 \mathrm{ft}$

SUSP. 15-lb weight

PRICE AA CURRENT METER No: 90JM-1 DATE RATED: 06-91

CURRENT METER EQUATION: $\mathrm{V}(\mathrm{m} / \mathrm{s})=\mathrm{Rev} / \mathrm{s}^{*} 0.667+0.006$

REMARKS:

Anchored at five verticals using the small boat. Depth integrated measurement by hand. Temperature and specific conductance measured by the Sea Bird, SeaCat profiler. Maximum transit rate was $8 \mathrm{~cm} / \mathrm{s}$ and the unmeasured zone was $0.17 \mathrm{~m}$.

\begin{tabular}{lccccccc}
\hline Vertical & $\begin{array}{c}\text { Distance } \\
\text { from } \\
\text { LEW } \\
(\mathbf{m})\end{array}$ & $\begin{array}{c}\text { Depth } \\
(\mathrm{m})\end{array}$ & $\begin{array}{c}\text { Mean } \\
\text { velocity } \\
(\mathbf{m} / \mathbf{s})\end{array}$ & $\begin{array}{c}\text { Discharge } \\
\left(\mathrm{m}^{3} / \mathrm{s}\right)\end{array}$ & $\begin{array}{c}\text { Temperature } \\
\left({ }^{\circ} \mathrm{C}\right)\end{array}$ & pH & $\begin{array}{c}\text { Specific } \\
\text { conductance } \\
(\mu \mathbf{S} / \mathbf{c m})\end{array}$ \\
\hline LEW & 0 & 0.0 & 0.00 & & & & \\
01 & 16 & 5.1 & 0.29 & 28 & 4.5 & -- & 150 \\
02 & 38 & 9.5 & 0.35 & 116 & 4.5 & -- & 148 \\
03 & 85 & 9.1 & 0.40 & 128 & 4.5 & -- & 149 \\
04 & 108 & 7.6 & 0.23 & 44 & 4.5 & -- & 149 \\
05 & 136 & 2.6 & 0.13 & 7 & 4.4 & - & 148 \\
REW & 152 & 0.0 & 0.00 & & & & \\
MEANS & & 6.5 & 0.32 & & & & \\
TOTAL & 152 & & & 324 & & & \\
\hline
\end{tabular}




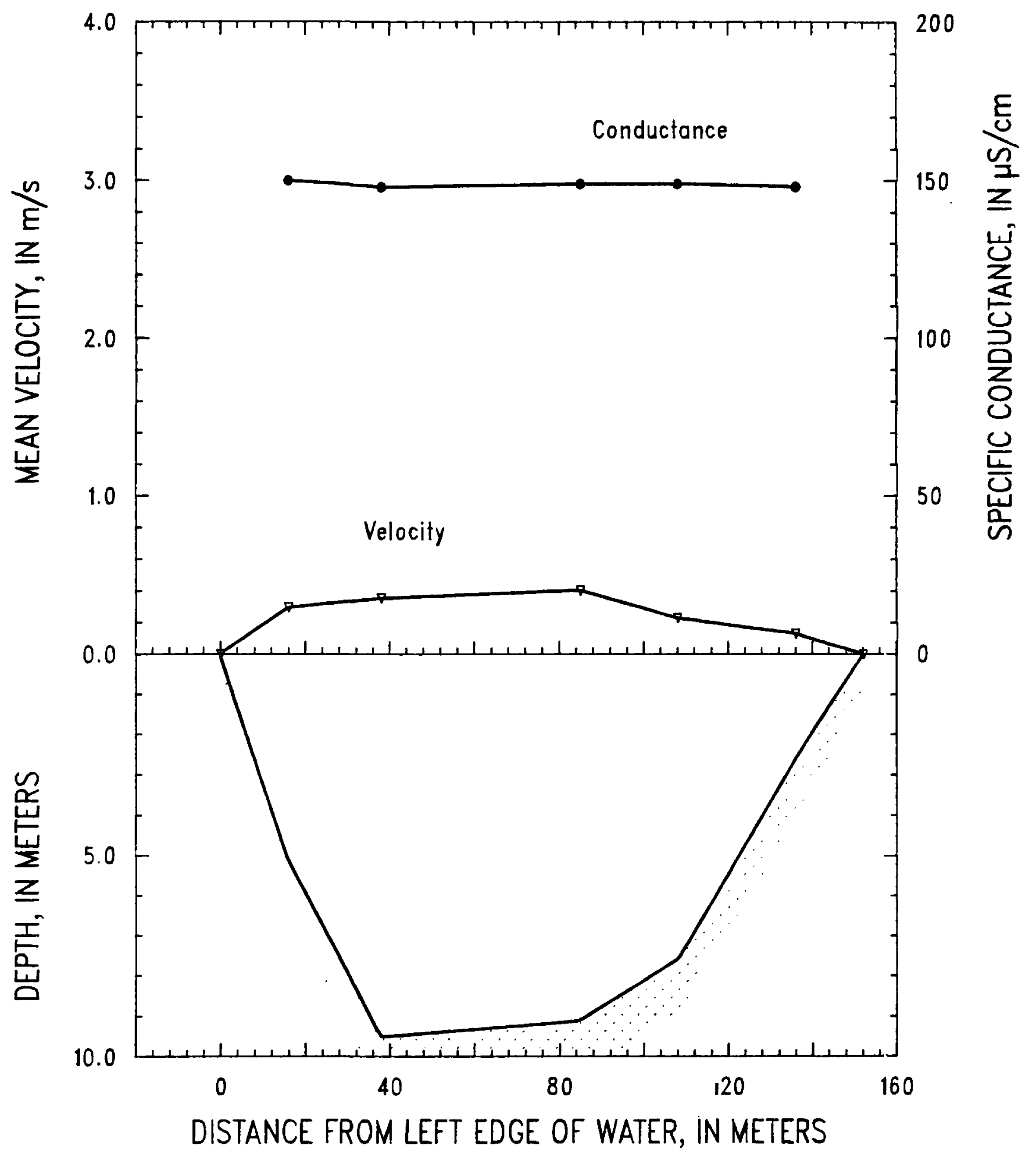

Figure 44. St. Croix River at Mile 0.0, Minnesota-Wisconsin, on April 11, 1992. 
SITE: Mississippi River near Pepin, Wis.-Mile 764.5

PARTY: Moody, Roth, and LeBoeuf

GAGE HEIGHT @ Lake City: $670.27 \mathrm{ft}$ GAGE HEIGHT @ Wabasha: $668.76 \mathrm{ft}$

RIVER SLOPE: $23 \times 10^{-6}$

SUSP. Bag sampler and $150-$ lb weight

PRICE AA CURRENT METER No: W-223906 DATE RATED: 06-91

CURRENT METER EQUATTON: $\mathrm{V}(\mathrm{m} / \mathrm{s})=\mathrm{Rev} / \mathrm{s} * 0.670+0.010$

REMARKS:

No depth-integrated sample was collected. The unmeasured zone was $0.66 \mathrm{~m}$. The discharge through

Dam 4 was 1,370 $\mathrm{m}^{3} / \mathrm{s}$ (Chippewa River joins Mississippi River between gaging site and Dam 4). Velocities were measured during 1 hour in the morning and the temperature, $\mathrm{pH}$, and specific conductance were maasured during 7 hours of collecting water samples by pumping at the same verticals-in the following order:

$7,5,6,4,3,2$, and 1 .

\begin{tabular}{|c|c|c|c|c|c|c|c|}
\hline Vertical & $\begin{array}{l}\text { Distance } \\
\text { from } \\
\text { LEW } \\
\text { (m) }\end{array}$ & $\begin{array}{l}\text { Depth } \\
\text { (m) }\end{array}$ & $\begin{array}{l}\text { Mean } \\
\text { velocity } \\
(\mathbf{m} / \mathbf{s})\end{array}$ & $\begin{array}{c}\text { Discharge } \\
\left(\mathrm{m}^{3} / \mathrm{s}\right)\end{array}$ & $\begin{array}{l}\text { Temperature } \\
\left({ }^{\circ} \mathrm{C}\right)\end{array}$ & pH & $\begin{array}{c}\text { Spectfic } \\
\text { conductance } \\
(\mu \mathrm{S} / \mathrm{cm})\end{array}$ \\
\hline LEW & 0 & 0.0 & 0.00 & & & & \\
\hline 01 & 61 & 2.6 & 0.37 & 57 & 7.3 & 8.6 & 513 \\
\hline 02 & 117 & 3.6 & 0.43 & 94 & 7.6 & 8.6 & 511 \\
\hline 03 & 183 & 3.9 & 0.43 & 95 & 8.2 & 8.5 & 513 \\
\hline 04 & 231 & 4.3 & 0.46 & 97 & 8.5 & 8.5 & 513 \\
\hline 05 & 281 & 4.5 & 0.52 & 136 & 8.0 & 8.6 & 513 \\
\hline 06 & 348 & 6.4 & 0.52 & 205 & 8.5 & 8.6 & 517 \\
\hline 07 & 403 & 8.9 & 0.53 & 222 & 7.3 & 8.6 & 513 \\
\hline$X 01$ & 443 & 3.2 & 0.45 & 41 & -- & - & - \\
\hline REW & 460 & 0.0 & 0.00 & & & & \\
\hline MEANS & & 4.3 & 0.48 & & & & \\
\hline TOTAL & 460 & & & 946 & & & \\
\hline
\end{tabular}




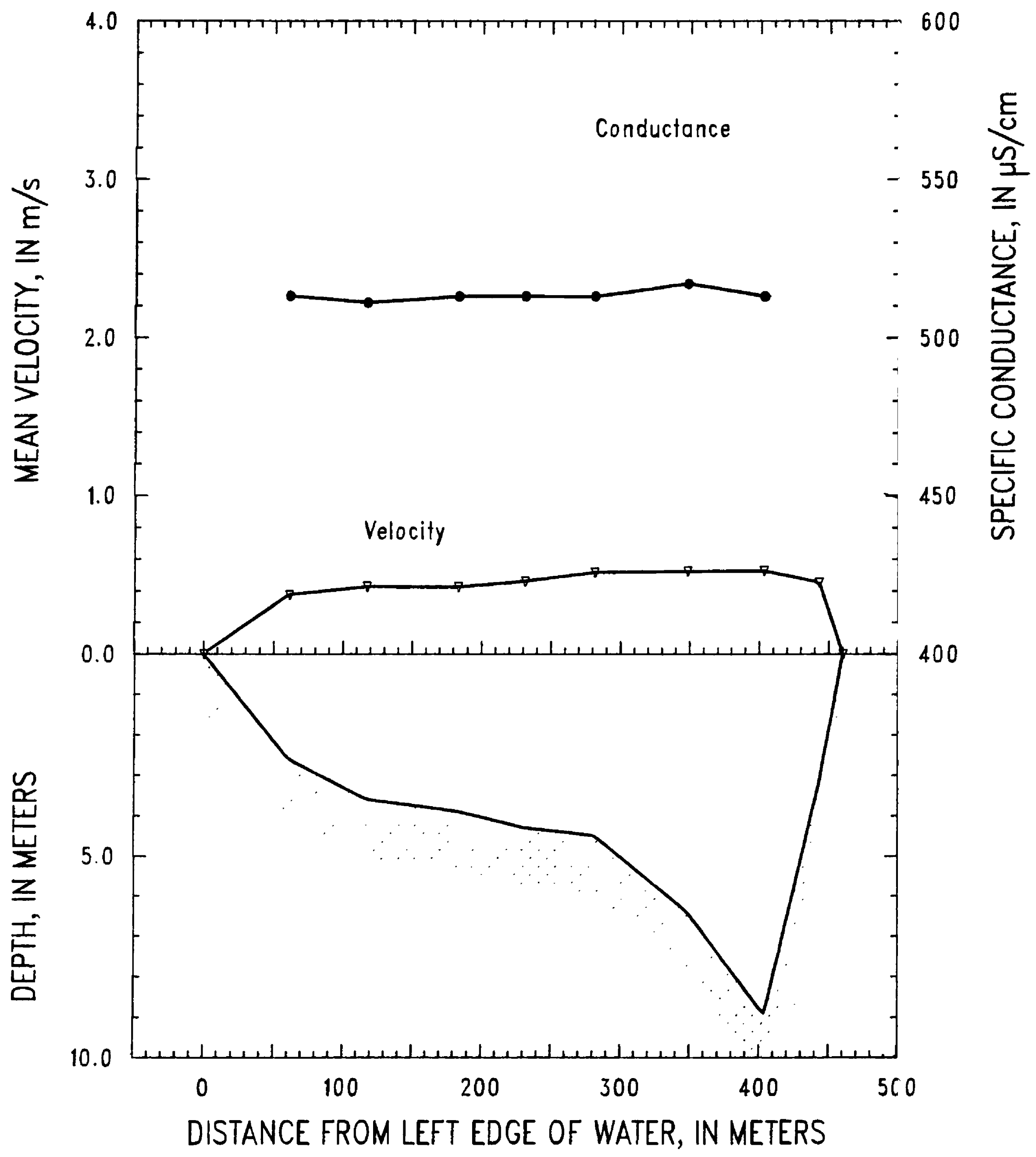

Figure 45. Mississippi River near Pepin, Wisconsin, on April 12, 1992. 
SITE: Chippewa River at Mile 2.0, Wis.

PARTY: Moody

GAGE HEIGHT @ Pool 4: $666.65 \mathrm{ft}$

SUSP. 15-lb weight

PRICE AA CURRENT METER No: 90-JM-1 DATE RATED: 06-91

CURRENT METER EQUATION: $V(\mathrm{~m} / \mathrm{s})=\operatorname{Rev} / \mathrm{s}^{*} 0.667+0.006$

REMARKS:

Discharge was measured from the small boat using the microwave system to measure distan ses from LEW to the anchored boat. Temperature and specific conductance were measured with LabComp instrument.

\begin{tabular}{lccccccc}
\hline Vertical & $\begin{array}{c}\text { Distance } \\
\text { from } \\
\text { LEW } \\
(\mathrm{m})\end{array}$ & $\begin{array}{c}\text { Depth } \\
(\mathrm{m})\end{array}$ & $\begin{array}{c}\text { Mean } \\
\text { velocity } \\
(\mathrm{m} / \mathrm{s})\end{array}$ & $\begin{array}{c}\text { Discharge } \\
\left(\mathrm{m}^{3} / \mathrm{s}\right)\end{array}$ & $\begin{array}{c}\text { Temperature } \\
\left({ }^{\circ} \mathrm{C}\right)\end{array}$ & $\begin{array}{c}\text { pH } \\
\begin{array}{c}\text { Specific } \\
\text { conductance } \\
(\mu \mathrm{S} / \mathrm{cm})\end{array}\end{array}$ \\
\hline LEW & 0 & 0.0 & 0.00 & & 4.6 & - & 115 \\
09 & 18 & 2.2 & 0.99 & 34 & 4.9 & -- & 114 \\
01 & 31 & 2.5 & 1.29 & 43 & 4.4 & -- & 117 \\
08 & 45 & 2.1 & 1.11 & 34 & 5.0 & -- & 117 \\
02 & 60 & 1.9 & 0.92 & 28 & 4.3 & -- & 117 \\
03 & 77 & 1.9 & 0.88 & 31 & 4.4 & -- & 120 \\
04 & 97 & 1.4 & 0.79 & 21 & 4.6 & -- & 120 \\
05 & 115 & 1.2 & 0.82 & 21 & 4.9 & -- & 122 \\
06 & 140 & 1.2 & 0.89 & 39 & 5.0 & -- & 127 \\
07 & 187 & 1.6 & 0.78 & 45 & 5.4 & -- & 131 \\
REW & 212 & 0.0 & 0.00 & & 4.8 & -- & 135 \\
MEANS & & 1.5 & 0.95 & & & & \\
TOTAL & 212 & & & 295 & & & \\
\hline
\end{tabular}




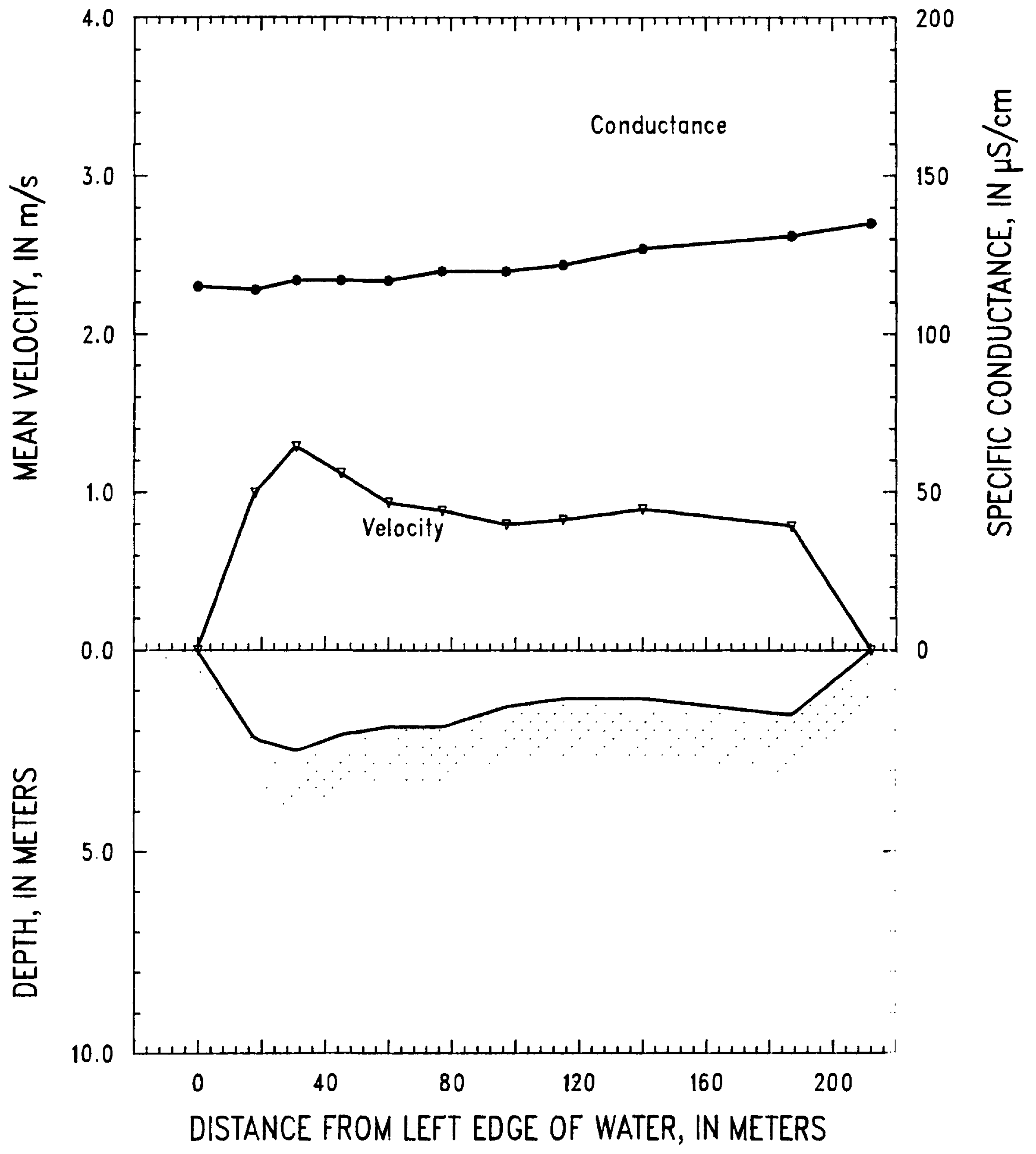

Figure 46. Chippewa River at Mile 2.0, Wisconsin, on April 12, 1992. 
SITE: Mississippi River at Trempealeau, Wis.-Mile 713.8

PARTY: Moody, Roth, and LeBoeuf

GAGE HEIGHT @ Dam 6 TW: 641.64ft GAGE HEIGHT @ Pool 7: $638.85 \mathrm{ft}$

RIVER SLOPE: $48.0 \times 10^{-6}$

SUSP. Bag sampler and 150-lb weight

PRICE AA CURRENT METER No: W-223906 DATE RATED: 06-91

CURRENT METER EQUATION: $\mathrm{V}(\mathrm{m} / \mathrm{s})=\operatorname{Rev} / \mathrm{s} * 0.670+0.010$

REMARKS:

Transit rate was $5.5 \mathrm{~cm} / \mathrm{s}$ and the nozzle was $5 / 16$ inch. Unmeasured zone was $0.66 \mathrm{~m}$.

\begin{tabular}{|c|c|c|c|c|c|c|c|}
\hline Vertical & $\begin{array}{c}\text { Distance } \\
\text { from } \\
\text { LEW } \\
\text { (m) }\end{array}$ & $\begin{array}{l}\text { Depth } \\
\text { (m) }\end{array}$ & $\begin{array}{c}\text { Mean } \\
\text { veloclty } \\
(\mathrm{m} / \mathrm{s})\end{array}$ & $\begin{array}{c}\text { Discharge } \\
\left(\mathrm{m}^{3} / \mathrm{s}\right)\end{array}$ & $\begin{array}{c}\text { Temperature } \\
\left({ }^{\circ} \mathrm{C}\right)\end{array}$ & pH & $\begin{array}{c}\text { Specific } \\
\text { conductance } \\
(\mu \mathrm{S} / \mathrm{cm})\end{array}$ \\
\hline LEW & 0 & 0.0 & 0.00 & & & & \\
\hline$X 06$ & 45 & 2.5 & 0.31 & 20 & - & - & -- \\
\hline $01 \mathrm{~A}, \mathrm{~B}$ & 50 & 2.7 & 0.41 & 28 & 6.0 & 8.4 & 392 \\
\hline 02A,B & 96 & 4.8 & 0.57 & 124 & 6.1 & 8.4 & 395 \\
\hline X05 & 141 & 4.7 & 0.57 & 72 & -- & -- & - \\
\hline $\mathrm{X} 11$ & 150 & 4.6 & 0.65 & 36 & - & -- &.- \\
\hline $03 A, B$ & 165 & 4.7 & 0.65 & 93 & 5.8 & 8.5 & 401 \\
\hline 04A,B & 211 & 4.6 & 0.67 & 105 & 5.8 & 8.5 & 411 \\
\hline$\times 09$ & 233 & 4.8 & 0.76 & 127 & -. & - & -- \\
\hline 05A,B & 281 & 5.2 & 0.69 & 131 & 5.7 & 8.5 & 419 \\
\hline $\mathrm{X08}$ & 306 & 5.7 & 0.72 & 116 & -- & - & -- \\
\hline 06A,B & 338 & 6.1 & 0.72 & 136 & 5.8 & 8.5 & 430 \\
\hline$x 07$ & 368 & 8.1 & 0.81 & 151 & - & - & -- \\
\hline $\mathrm{X} 10$ & 384 & 8.0 & 0.66 & 73 & - & -- & - \\
\hline 07A,B & 396 & 7.8 & 0.67 & 115 & 5.7 & 8.5 & 440 \\
\hline REW & 428 & 0.0 & 0.00 & & & & \\
\hline MEANS & & 4.7 & 0.66 & & & & \\
\hline TOTAL & 428 & & & 1,328 & & & \\
\hline
\end{tabular}




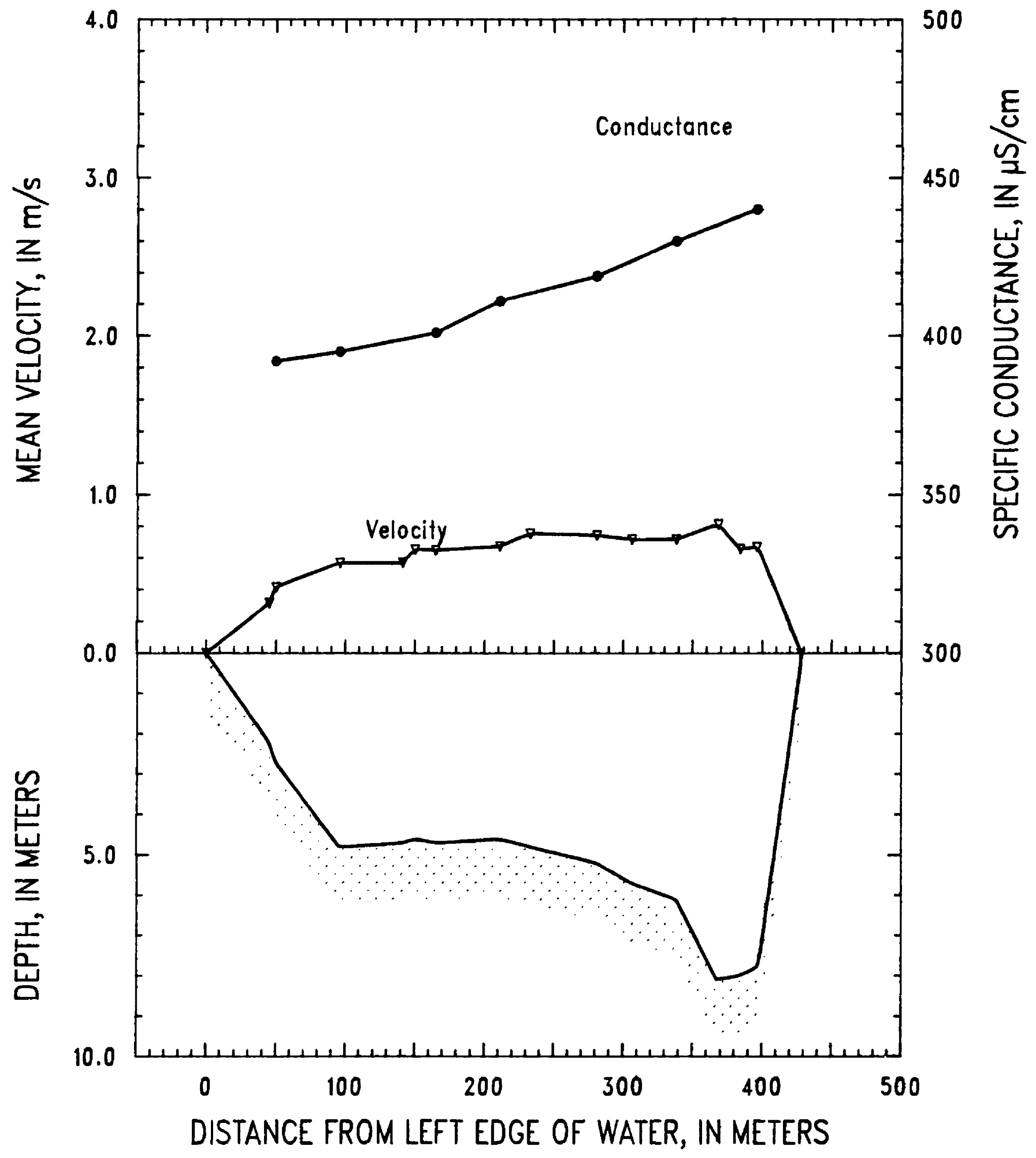

Figure 47. Mississippi River at Trempealeau, Wisconsin, on April 14, 1992. 
SITE: Mississippi River below Lock and Dam 9, Wis.-Mile 639.7

04-17-92

PARTY: Moody, Antweiler, and LeBoeuf

GAGE HEIGHT @ Dam 9 TW: 617.75 ft GAGE HEIGHT @ Pool 10: 610.15 ft

RIVER SLOPE: $43.6 \times 10^{-6}$

SUSP. Bag sampler and 150-lb weight

PRICE AA CURRENT METER No: W-223906 DATE RATED: 06-91

CURRENT METER EQUATION: $V(\mathrm{~m} / \mathrm{s})=\operatorname{Rev} / \mathrm{s}^{*} 0.670+0.010$

REMARKS:

Transit rate was $6.0 \mathrm{~cm} / \mathrm{s}$ and the nozzle was $5 / 16$ inch. Discharge from Dam 9 was $1,490 \mathrm{~m}^{3} / \mathrm{s}$.

\begin{tabular}{|c|c|c|c|c|c|c|c|}
\hline Vertical & $\begin{array}{l}\text { Distance } \\
\text { from } \\
\text { LEW } \\
\text { (m) }\end{array}$ & $\begin{array}{l}\text { Depth } \\
\text { (m) }\end{array}$ & $\begin{array}{c}\text { Mean } \\
\text { veiocity } \\
(\mathrm{m} / \mathrm{s})\end{array}$ & $\begin{array}{c}\text { Discharge } \\
\left(\mathrm{m}^{3} / \mathrm{s}\right)\end{array}$ & $\begin{array}{c}\text { Temperature } \\
\left({ }^{\circ} \mathrm{C}\right)\end{array}$ & pH & $\begin{array}{c}\text { Spechific } \\
\text { conductance } \\
(\mu \mathrm{S} / \mathrm{cm})\end{array}$ \\
\hline LEW & 0 & 0.0 & 0.00 & & & & \\
\hline$X 01$ & 40 & 2.7 & 0.41 & 29 & $\because$ & -- & -. \\
\hline $01 \mathrm{~A}, \mathrm{~B}$ & 52 & 3.1 & 0.41 & 45 & 6.6 & 8.9 & 389 \\
\hline 02A,B & 111 & 4.6 & 0.58 & 122 & 6.6 & 8.9 & 391 \\
\hline$X_{02}$ & 144 & 5.4 & 0.62 & 85 & - & -- & -- \\
\hline 03A,B & 162 & 5.9 & 0.77 & 174 & 6.6 & 8.9 & 392 \\
\hline $04 \mathrm{~A}, \mathrm{~B}$ & 221 & 6.8 & 0.78 & 193 & 6.6 & 8.9 & 397 \\
\hline $\mathrm{X} 03$ & 235 & 7.0 & 0.79 & 161 & - & -- & -- \\
\hline 05A,B & 279 & 6.9 & 0.81 & 286 & 6.7 & 8.7 & 401 \\
\hline 06A,B & 337 & 6.6 & 0.79 & 162 & 6.5 & 8.3 & 412 \\
\hline $\mathrm{X04}$ & 341 & 6.5 & 0.72 & 134 & - & -- & - \\
\hline 07A,B & 394 & 4.9 & 0.67 & 129 & 6.8 & 8.2 & 417 \\
\hline X05 & 419 & 4.1 & 0.61 & 71 & & & \\
\hline REW & 450 & 0.0 & 0.00 & & & & \\
\hline MEANS & & 5.0 & 0.70 & & & & \\
\hline TOTAL & 450 & & & 1,589 & & & \\
\hline
\end{tabular}




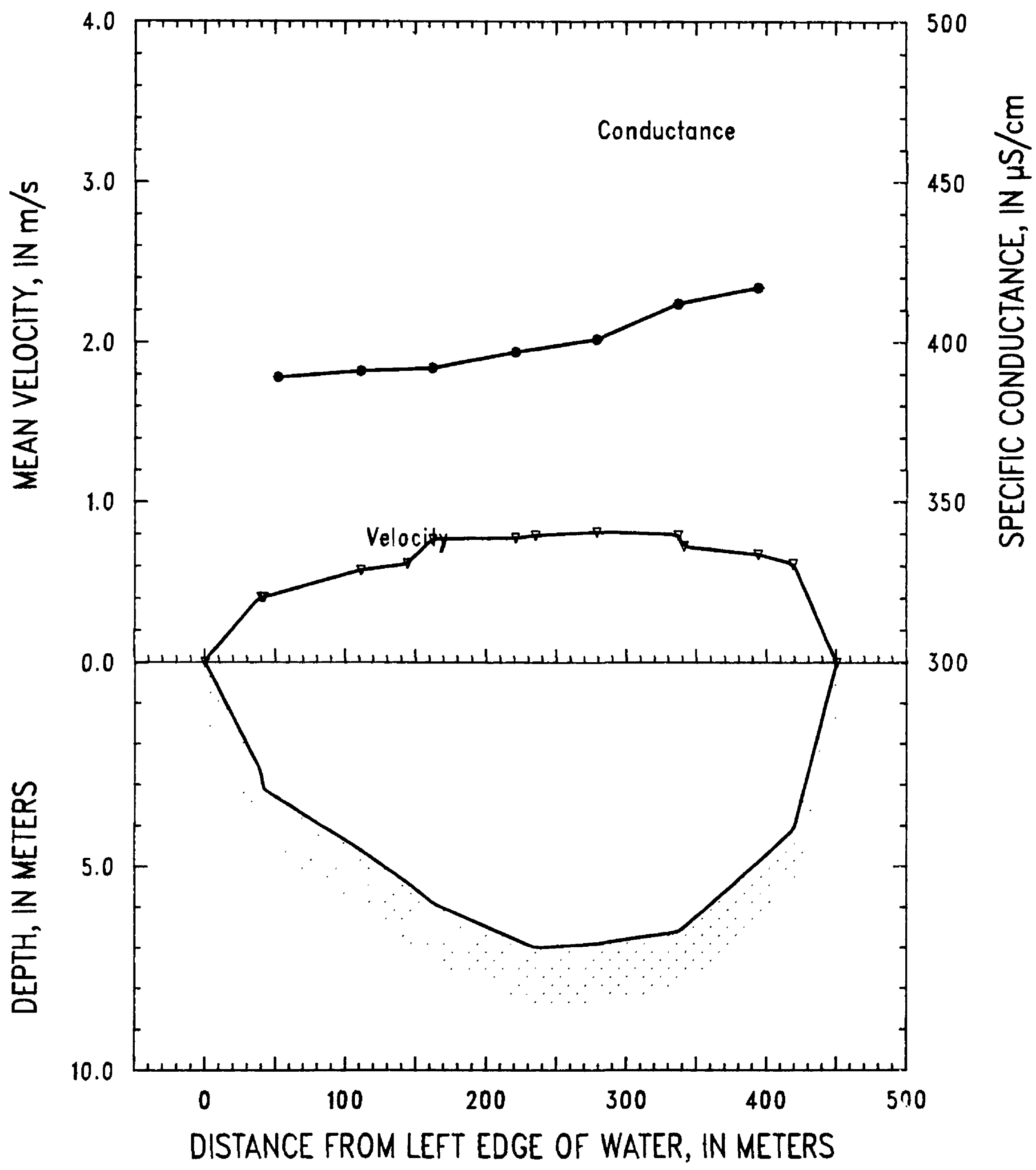

Figure 48. Mississippi River below Lock and Dam 9, Wisconsin, on April 17, 1992. 
SITE: Mississippi River at Clinton, Iowa-Mile 520.3

04-19-92

PARTY: Moody, Antweiler, and LeBoeuf

GAGE HEIGHT @ Dam 13 TW: $578.42 \mathrm{ft} \quad$ GAGE HEIGHT @ Pool 14: $571.83 \mathrm{ft}$

RIVER SLOPE: $41.6 \times 10^{-6}$

SUSP. Bag sampler and $150-\mathrm{lb}$ weight

PRICE AA CURRENT METER No: W-223906 DATE RATED: 06-91

CURRENT METER EQUATION: $\mathrm{V}(\mathrm{m} / \mathrm{s})=\operatorname{Rev} / \mathrm{s}^{*} 0.670+0.010$

REMARKS:

Transit rate was $6.0 \mathrm{~cm} / \mathrm{sec}$ and the nozzle was $1 / 4$ inch. The unmeasured zone was $0.66 \mathrm{~m}$. Verticals 1 and 2 were combined and collected at a location about halfway between the two verticals. The orde- of the verticals was $8,7,3,1+2,4,5$, and 6 .

\begin{tabular}{|c|c|c|c|c|c|c|c|}
\hline Verticai & $\begin{array}{l}\text { Distence } \\
\text { from } \\
\text { LEW } \\
\text { (m) }\end{array}$ & $\begin{array}{l}\text { Depth } \\
\text { (m) }\end{array}$ & $\begin{array}{c}\text { Mean } \\
\text { velocity } \\
(\mathrm{m} / \mathrm{s})\end{array}$ & $\begin{array}{c}\text { Discharge } \\
\left(\mathrm{m}^{3} / \mathrm{s}\right)\end{array}$ & $\begin{array}{c}\text { Temperature } \\
\left({ }^{\circ} \mathrm{C}\right)\end{array}$ & $\mathrm{pH}$ & $\begin{array}{c}\text { Specific } \\
\text { conductance } \\
(\mu \mathrm{S} / \mathrm{cm})\end{array}$ \\
\hline LEW & 0 & 0.0 & 0.00 & & & & \\
\hline $1+2 \mathrm{~A}$ & 66 & 2.7 & 0.26 & 25 & 11.1 & 8.7 & 395 \\
\hline $1+2 B$ & 72 & 3.2 & 0.39 & 15 & - & -- & - \\
\hline$X 01$ & 90 & 3.9 & 0.47 & 68 & -- & -- & - \\
\hline $\mathrm{X02}$ & 146 & 6.9 & 0.59 & 149 & - & -- & - \\
\hline 03A,B & 163 & 7.8 & 0.62 & 128 & 11.4 & 8.8 & 378 \\
\hline $\mathrm{X} 03$ & 199 & 8.2 & 0.66 & 128 & -- & -- & -. \\
\hline 04A,B & 210 & 8.3 & 0.69 & 167 & 11.4 & 8.8 & 376 \\
\hline 05A,B & 257 & 9.0 & 0.82 & 329 & 11.0 & 8.8 & 377 \\
\hline X04 & 299 & 9.8 & 0.90 & 261 & - & -- & -- \\
\hline 06A,B & 316 & 10.0 & 0.89 & 267 & 10.7 & 8.9 & 376 \\
\hline X05 & 359 & 10.1 & 0.92 & 278 & -. & - & - \\
\hline 07A,B & 376 & 10.1 & 0.87 & 249 & 10.5 & 8.9 & 386 \\
\hline $\mathrm{X} 07$ & 416 & 8.8 & 0.66 & 152 & -- & - & -- \\
\hline 08A,B & 428 & 8.2 & 0.53 & 71 & 10.1 & 9.0 & 403 \\
\hline $\mathrm{X} 06$ & 449 & 5.6 & 0.26 & 34 & & & \\
\hline REW & 475 & 0.0 & 0.00 & & & & \\
\hline MEANS & & 6.8 & 0.71 & & & & \\
\hline TOTAL & 475 & & & 2,320 & & & \\
\hline
\end{tabular}




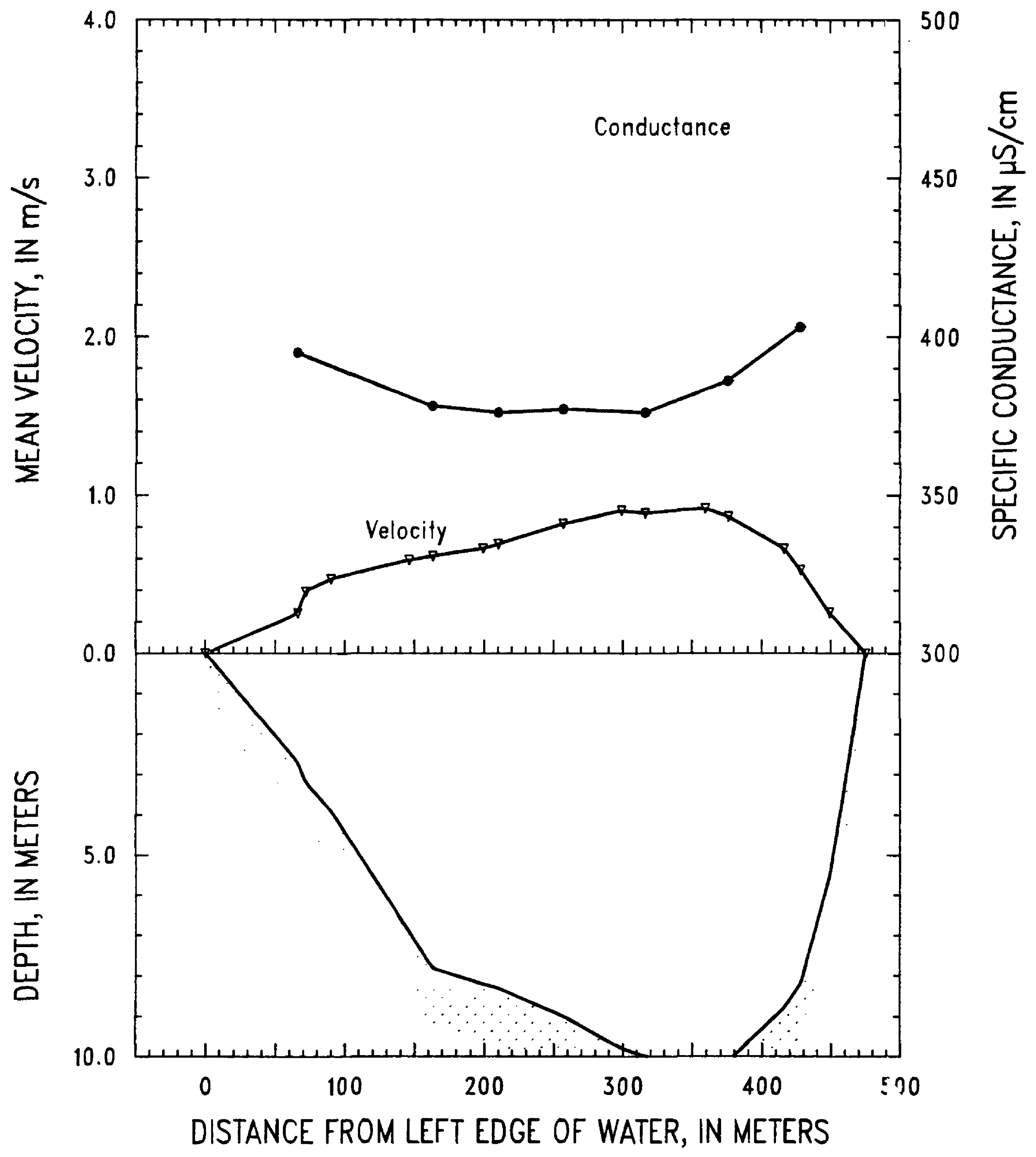

Figure 49. Mississippi River at Clinton, lowa, on April 19, 1992. 
SITE: Mississippi River at Keokuk, Iowa-Mile 363.1

PARTY: Moody, Brown, Krest, and LeBoeuf

GAGE HEIGHT @ Dam 19 TW: 489.06 ft GAGE HEIGHT @ Pool 20: $481.12 \mathrm{ft}$

RIVER SLOPE: $71.6 \times 10^{-6}$

SUSP. Bag sampler and 150-lb weight

PRICE AA CURRENT METER No: W-223906 DATE RATED: 06-91

CURRENT METER EQUATION: $V(\mathrm{~m} / \mathrm{s})=\operatorname{Rev} / \mathrm{s}^{*} 0.670+0.010$

REMARKS:

Transit rate was $8.5 \mathrm{~cm} / \mathrm{s}$ and the nozzle was $5 / 16$ inch. Unmeasured zone was $0.66 \mathrm{~m}$. The $\mathrm{pH}$ meter was calibrated at the start of the day but the readings at the last vertical (vertical 1) were much more variable $(7.68,7.04$, and 7.12$)$ so that the meter was recalibrated and the readings increased to 8.51 at vertical 1 . Anchored at vertical 1 to prevent prop wash from stirring up fine sediment in shallow water and being col'acted in the pumped sample. Order of the verticals was $7,5,6,4,3,2$, and 1 .

\begin{tabular}{|c|c|c|c|c|c|c|c|}
\hline Vertical & $\begin{array}{l}\text { Distance } \\
\text { from } \\
\text { LEW } \\
(\mathbf{m})\end{array}$ & $\begin{array}{l}\text { Depth } \\
\text { (m) }\end{array}$ & $\begin{array}{l}\text { Mean } \\
\text { veloclty } \\
(\mathrm{m} / \mathrm{s})\end{array}$ & $\begin{array}{c}\text { Discharge } \\
\left(\mathrm{m}^{3} / \mathrm{s}\right)\end{array}$ & $\begin{array}{c}\text { Temperature } \\
\left({ }^{\circ} \mathrm{C}\right)\end{array}$ & pH & $\begin{array}{c}\text { Speclfic } \\
\text { conductance } \\
(\mu \mathrm{S} / \mathrm{cm})\end{array}$ \\
\hline LEW & 0 & 0.0 & 0.00 & & & & \\
\hline$X 07$ & 64 & 4.2 & 0.67 & 103 & -- & -- & -- \\
\hline $01 \mathrm{~A}, \mathrm{~B}$ & 73 & 4.4 & 0.72 & 172 & 11.2 & 8.5 & 439 \\
\hline 02A,B & 173 & 4.9 & 0.96 & 311 & 11.0 & 7.6 & 436 \\
\hline $\mathrm{X06}$ & 205 & 5.6 & 1.00 & 274 & - & -- & - \\
\hline 03A,B & 271 & 5.4 & 0.96 & 247 & 10.9 & 7.6 & 430 \\
\hline $\mathrm{X08}$ & 300 & 5.6 & 1.10 & 268 & -- & -- & -- \\
\hline X05 & 358 & 5.8 & 1.22 & 371 & -- & -- & -- \\
\hline $04 A, B$ & 405 & 5.8 & 1.21 & 316 & 11.0 & 7.5 & 428 \\
\hline X04 & 448 & 6.1 & 1.19 & 337 & - & -- & - \\
\hline 05A,B & 498 & 6.6 & 1.16 & 282 & 10.7 & 7.8 & 423 \\
\hline$\times 03$ & 522 & 6.8 & 1.15 & 471 & - & - & -- \\
\hline 06A,B & 619 & 6.4 & 1.18 & 558 & 11.1 & 7.7 & 421 \\
\hline $\mathrm{X} 02$ & 670 & 5.5 & 1.08 & 280 & -- & -- & -- \\
\hline$X 01$ & 713 & 5.0 & 0.84 & 109 & -- & -- & -- \\
\hline 07A,B & 722 & 4.6 & 0.70 & 124 & 11.1 & 7.8 & 412 \\
\hline REW & 790 & 0.0 & 0.00 & & & & \\
\hline MEANS & & 5.1 & 1.05 & & & & \\
\hline TOTAL & 790 & & & 4,222 & & & \\
\hline
\end{tabular}




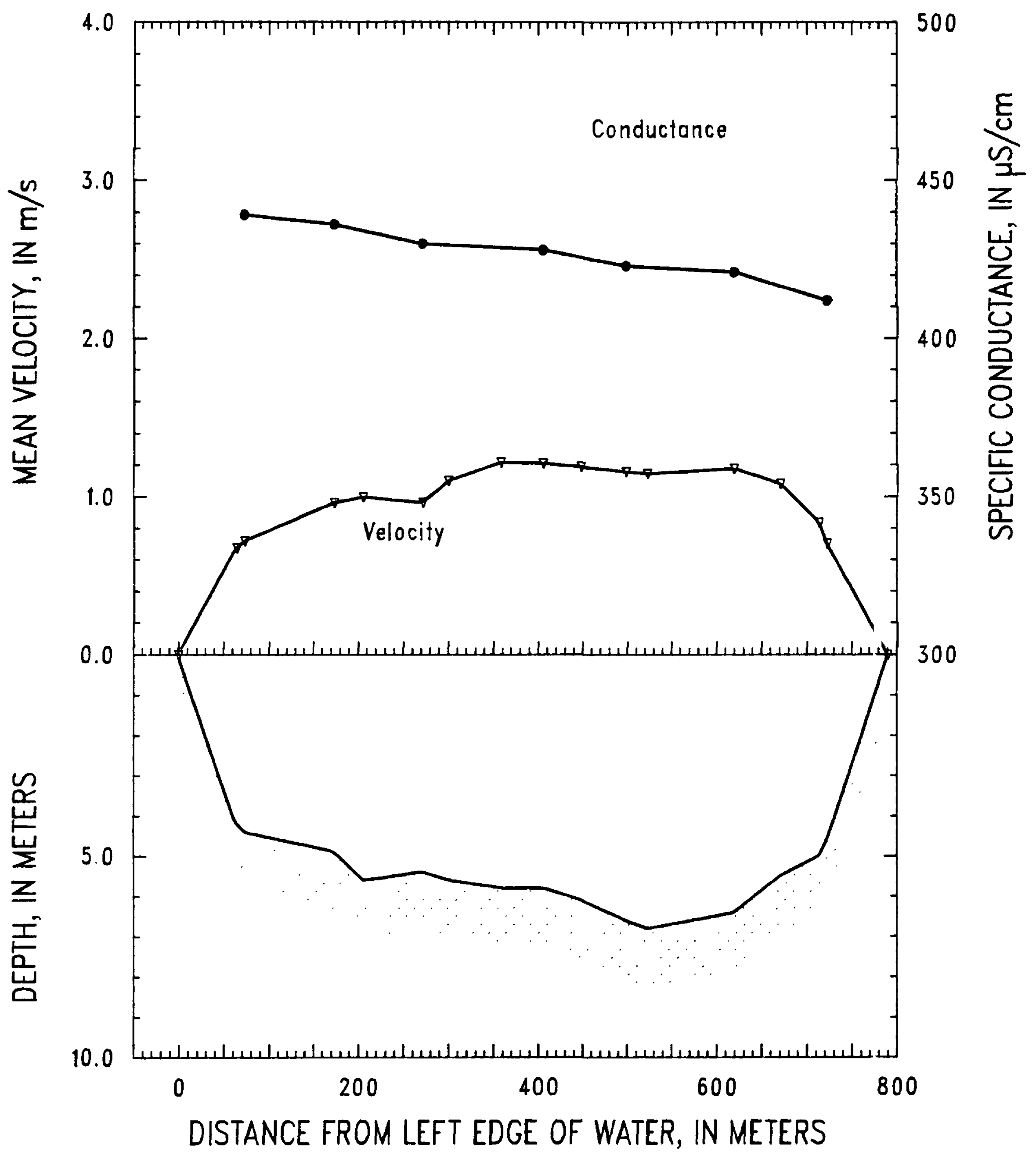

Figure 50. Mississippi River at Keokuk, lowa, on April 23, 1992. 
SITE: Mississippi River near Winfield, Mo.-Mile 239.2

04-26-92

PARTY: Moody, Krest, and Simoneaux

GAGE HEIGHT @ Dam 25 TW: $431.78 \mathrm{ft}$ GAGE HEIGHT @ Grafton: $420.99 \mathrm{ft}$

RIVER SLOPE: $88.9 \times 10^{-6}$

SUSP. Bag sampler and 150 -lb weight

PRICE AA CURRENT METER No: W-223906 DATE RATED: 06-91

CURRENT METER EQUATION: $V(\mathrm{~m} / \mathrm{s})=\operatorname{Rev} / \mathrm{s}^{*} 0.670+0.010$

REMARKS:

Transit rate was $11.0 \mathrm{~cm} / \mathrm{s}$ and nozzle was $1 / 4 \mathrm{inch}$. Unmeasured zone was $0.66 \mathrm{~m}$. Order of verticals was $1,2,3,7,6,5,4,8,9$, and 10 .

\begin{tabular}{|c|c|c|c|c|c|c|c|}
\hline Vertical & $\begin{array}{l}\text { Distance } \\
\text { from } \\
\text { LEW } \\
\text { (m) }\end{array}$ & $\begin{array}{l}\text { Depth } \\
\text { (m) }\end{array}$ & $\begin{array}{c}\text { Mean } \\
\text { velocity } \\
(\mathrm{m} / \mathrm{s})\end{array}$ & $\begin{array}{c}\text { Discharge } \\
\left(\mathrm{m}^{3} / \mathrm{s}\right)\end{array}$ & $\begin{array}{c}\text { Temperature } \\
\left({ }^{\circ} \mathrm{C}\right)\end{array}$ & pH & $\begin{array}{c}\text { Specific } \\
\text { conductance } \\
(\mu \mathrm{S} / \mathrm{cm})\end{array}$ \\
\hline LEW & 0 & 0.0 & 0.00 & & & & \\
\hline $01 \mathrm{~A}$ & 49 & 8.4 & 0.88 & 221 & 10.4 & 8.1 & 441 \\
\hline 01B & 60 & 10.4 & 1.08 & 259 & -- & - & -- \\
\hline $02 \mathrm{~A}, \mathrm{~B}$ & 95 & 11.3 & 1.32 & 342 & 10.3 & 7.9 & 441 \\
\hline $\mathrm{X} 02$ & 106 & 11.2 & 1.35 & 417 & -- & -- & - \\
\hline 03A,B & 150 & 10.6 & 1.20 & 489 & 10.2 & 7.8 & 444 \\
\hline X03 & 183 & 10.8 & 1.32 & 450 & - & - & -- \\
\hline $04 \mathrm{~A}, \mathrm{~B}$ & 213 & 10.1 & 1.25 & 390 & 10.3 & 8.3 & 451 \\
\hline $\mathrm{X04}$ & 245 & 10.0 & 1.15 & 294 & -- & - & - \\
\hline 05A,B & 264 & 9.8 & 1.12 & 455 & 10.3 & 8.2 & 450 \\
\hline $06 \mathrm{~A}, \mathrm{~B}$ & 328 & 8.5 & 1.06 & 366 & 10.5 & 8.2 & 449 \\
\hline X05 & 345 & 8.3 & 1.13 & 225 & -- & - & -- \\
\hline $07 \mathrm{~A}, \mathrm{~B}$ & 376 & 7.7 & 1.00 & 358 & 10.4 & 8.0 & 445 \\
\hline 08A, B & 438 & 6.3 & 0.96 & 358 & 10.5 & 8.3 & 448 \\
\hline 09A,B & 495 & 5.7 & 0.91 & 213 & 10.6 & 8.3 & 448 \\
\hline X06 & 520 & 5.9 & 0.78 & 76 & - & -- & - \\
\hline $10 \mathrm{~A}, \mathrm{~B}$ & 528 & 5.8 & 0.90 & 157 & 10.7 & 8.3 & 450 \\
\hline REW & 580 & 0.0 & 0.00 & & & & \\
\hline MEANS & & 7.9 & 1.11 & & & & \\
\hline TOTAL & 580 & & & 5,070 & & & \\
\hline
\end{tabular}




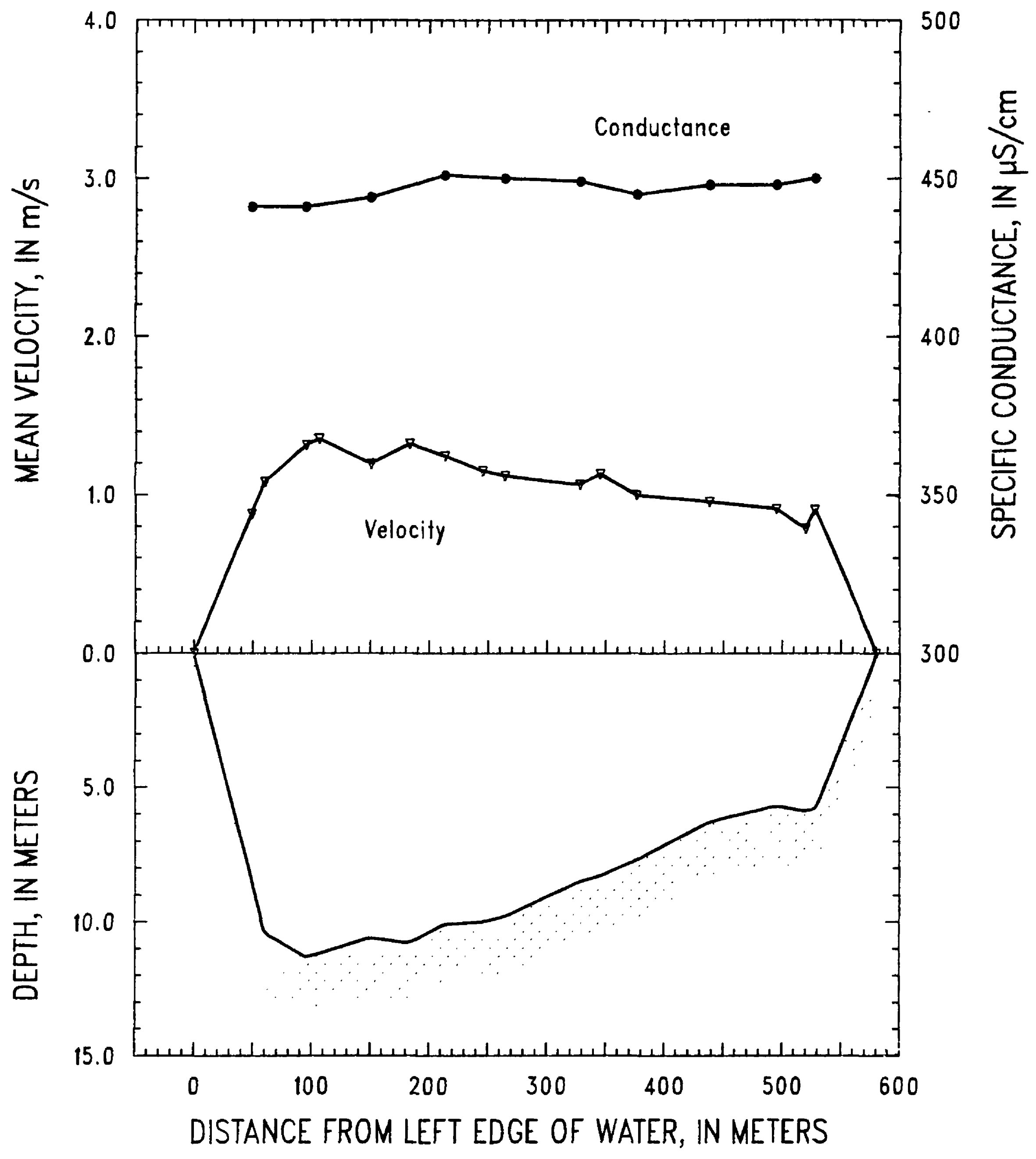

Figure 51. Mississippi River near Winfield, Missouri, on April 26, 1992. 
SITE: Illinois River at Hardin, Ill.-Mile 21.8

PARTY: Moody, Krest, and Simoneaux

GAGE HEIGHT @ Meredosia: $446.5 \mathrm{ft}$ GAGE HEIGHT @ Grafton: $421.0 \mathrm{ft}$

RIVER SLOPE: $97.0 \times 10^{-6}$

SUSP. Bag sampler and 150-lb weight

PRICE AA CURRENT METER No: W-223906 DATE RATED: 06-91

CURRENT METER EQUATION: $V(\mathrm{~m} / \mathrm{s})=\mathrm{Rev} / \mathrm{s}^{*} 0.670+0.010$

REMARKS:

Transit rate was $5.0 \mathrm{~cm} / \mathrm{s}$ and nozzle was $5 / 16 \mathrm{inch}$. Unmeasured zone was $0.66 \mathrm{~m}$. Order of verticals was $5,1,3,4$, and 2 .

\begin{tabular}{|c|c|c|c|c|c|c|c|}
\hline Vertical & $\begin{array}{l}\text { Distance } \\
\text { from } \\
\text { LEW } \\
\text { (m) }\end{array}$ & $\begin{array}{l}\text { Depth } \\
\text { (m) }\end{array}$ & $\begin{array}{l}\text { Mean } \\
\text { velocity } \\
(\mathrm{m} / \mathrm{s})\end{array}$ & $\begin{array}{c}\text { Discharge } \\
\left(\mathrm{m}^{3} / \mathrm{s}\right)\end{array}$ & $\begin{array}{c}\text { Temperature } \\
\left({ }^{\circ} \mathrm{C}\right)\end{array}$ & pH & $\begin{array}{c}\text { Specific } \\
\text { conductance } \\
(\mu \mathrm{S} / \mathrm{cm})\end{array}$ \\
\hline LEW & 0 & 0.0 & & & & & \\
\hline 01A,B & 38 & 3.2 & 0.51 & 51 & 11.9 & 8.2 & 767 \\
\hline X05 & 62 & 4.5 & 0.58 & 82 & - & - & -.. \\
\hline $02 \mathrm{~A}, \mathrm{~B}$ & 101 & 4.8 & 0.65 & 83 & 12.7 & 8.2 & 777 \\
\hline $3 \mathrm{~B} 2$ & 115 & 5.0 & 0.63 & 49 & $\ldots$ & $\ldots$ & - \\
\hline $03 \mathrm{~A}, \mathrm{~B}$ & 132 & 5.1 & 0.69 & 62 & 11.9 & 8.2 & 779 \\
\hline $\mathrm{X} 03$ & 150 & 5.8 & 0.71 & 85 & - & - & $\cdots$ \\
\hline X06 & 173 & 6.8 & 0.73 & 74 & $\ldots$ & -. & - \\
\hline 04A,B & 180 & 7.0 & 0.71 & 57 & 12.0 & 8.2 & 780 \\
\hline $\mathrm{X} 02$ & 196 & 6.9 & 0.70 & 99 & - & - & --- \\
\hline$\times 07$ & 221 & 6.8 & 0.72 & 80 & - & - & -- \\
\hline $05 \mathrm{~A}, \mathrm{~B}$ & 229 & 6.5 & 0.71 & 53 & 11.8 & 7.8 & 778 \\
\hline$X_{01}$ & 244 & 5.4 & 0.66 & 85 &.- & $\ldots$ & -- \\
\hline REW & 277 & 0.0 & & & & & \\
\hline MEANS & & 4.7 & 0.66 & & & & \\
\hline TOTAL & 277 & & & 859 & & & \\
\hline
\end{tabular}




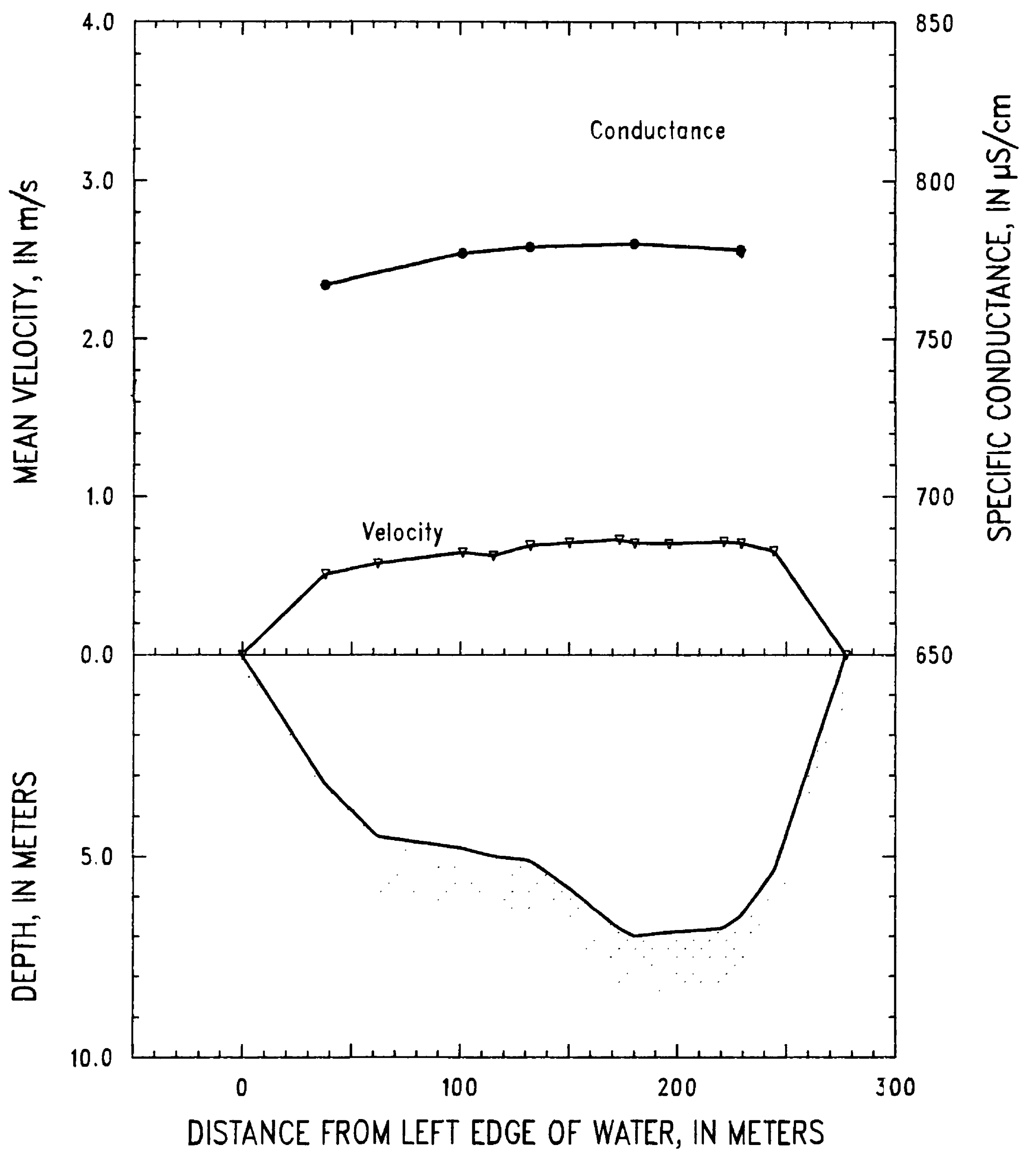

Figure 52. Illinois River at Hardin, Illinois, on April 27, 1992. 
SITE: Missouri River at St. Charles, Mo.--Mile 29.4

PARTY: Moody, Krest, and Simoneaux

GAGE HEIGHT @ Hermann: 497.86 ft GAGE HEIGHT @ St. Charles: $442.10 \mathrm{ft}$

RIVER SLOPE: $149 \times 10^{-6}$

SUSP. Bag sampler and 150-lb weight

PRICE AA CURRENT METER No: W-223906 DATE RATED: 06-91

CURRENT METER EQUATION: $V(\mathrm{~m} / \mathrm{s})=\operatorname{Rev} / \mathrm{s}^{*} 0.670+0.010$

REMARKS:

Transit rate was $8.0 \mathrm{~cm} / \mathrm{s}$ and the nozzle was $3 / 16$ inch for verticals $8,7,6$, and 5 , but was changed to $14.0 \mathrm{~cm} / \mathrm{s}$ and 1/4-inch nozzle when numerous nozzle blocks were encountered at vertical 5 and vertical 4 . The unmeasured zone was $0.66 \mathrm{~m}$.

\begin{tabular}{|c|c|c|c|c|c|c|c|}
\hline Vertical & $\begin{array}{l}\text { Distance } \\
\text { from } \\
\text { LEW } \\
\text { (m) }\end{array}$ & $\begin{array}{l}\text { Depth } \\
\text { (m) }\end{array}$ & $\begin{array}{l}\text { Mean } \\
\text { velocity } \\
(\mathrm{m} / \mathrm{s})\end{array}$ & $\begin{array}{c}\text { Discharge } \\
\left(\mathrm{m}^{3} / \mathrm{s}\right)\end{array}$ & $\begin{array}{c}\text { Temperature } \\
\left({ }^{\circ} \mathrm{C}\right)\end{array}$ & pH & $\begin{array}{c}\text { Spectilc } \\
\text { conductance } \\
(\mu \mathrm{S} / \mathrm{cm})\end{array}$ \\
\hline LEW & 0 & 0.0 & 0.00 & & & & \\
\hline $01 \mathrm{~A}, \mathrm{~B}$ & 45 & 3.8 & 0.65 & 117 & 13.5 & 7.9 & 399 \\
\hline $02 \mathrm{~A}, \mathrm{~B}$ & 95 & 4.4 & 1.14 & 230 & 14.1 & 7.9 & 402 \\
\hline 03A,B & 137 & 5.5 & 1.09 & 177 & 13.4 & 7.9 & 401 \\
\hline $\mathrm{X} 02$ & 154 & 5.7 & 1.35 & 161 & .- & -- & -- \\
\hline $04 \mathrm{~A}, \mathrm{~B}$ & 179 & 6.6 & 1.69 & 190 & 13.3 & 7.9 & 399 \\
\hline $04 R$ & 188 & 7.0 & 1.78 & 124 & - & -- & -- \\
\hline$X 03$ & 199 & 7.7 & 1.83 & 204 & - & -- & -. \\
\hline $5 \mathrm{AR}$ & 217 & 7.7 & 1.86 & 172 & -- & - & - \\
\hline 05B & 223 & 7.7 & 1.89 & 80 & 13.1 & 7.9 & 398 \\
\hline $05 \mathrm{~A}$ & 228 & 7.5 & 1.87 & 358 & -- & -- & -- \\
\hline 06A,B & 274 & 7.4 & 1.62 & 360 & 12.8 & 7.9 & 395 \\
\hline X04 & 288 & 6.5 & 1.76 & 280 & -- & -- & - \\
\hline 07A,B & 323 & 6.8 & 1.78 & 454 & 12.9 & 7.9 & 397 \\
\hline $08 \mathrm{~A}, \mathrm{~B}$ & 363 & 8.2 & 1.57 & 283 & 12.4 & 8.0 & 395 \\
\hline$X 56$ & 367 & 8.3 & 1.70 & 368 & -- & - & -- \\
\hline REW & 415 & 0.0 & 0.00 & & & & \\
\hline MEANS & & 5.6 & 1.54 & & & & \\
\hline TOTAL & 415 & & & 3,557 & & & \\
\hline
\end{tabular}




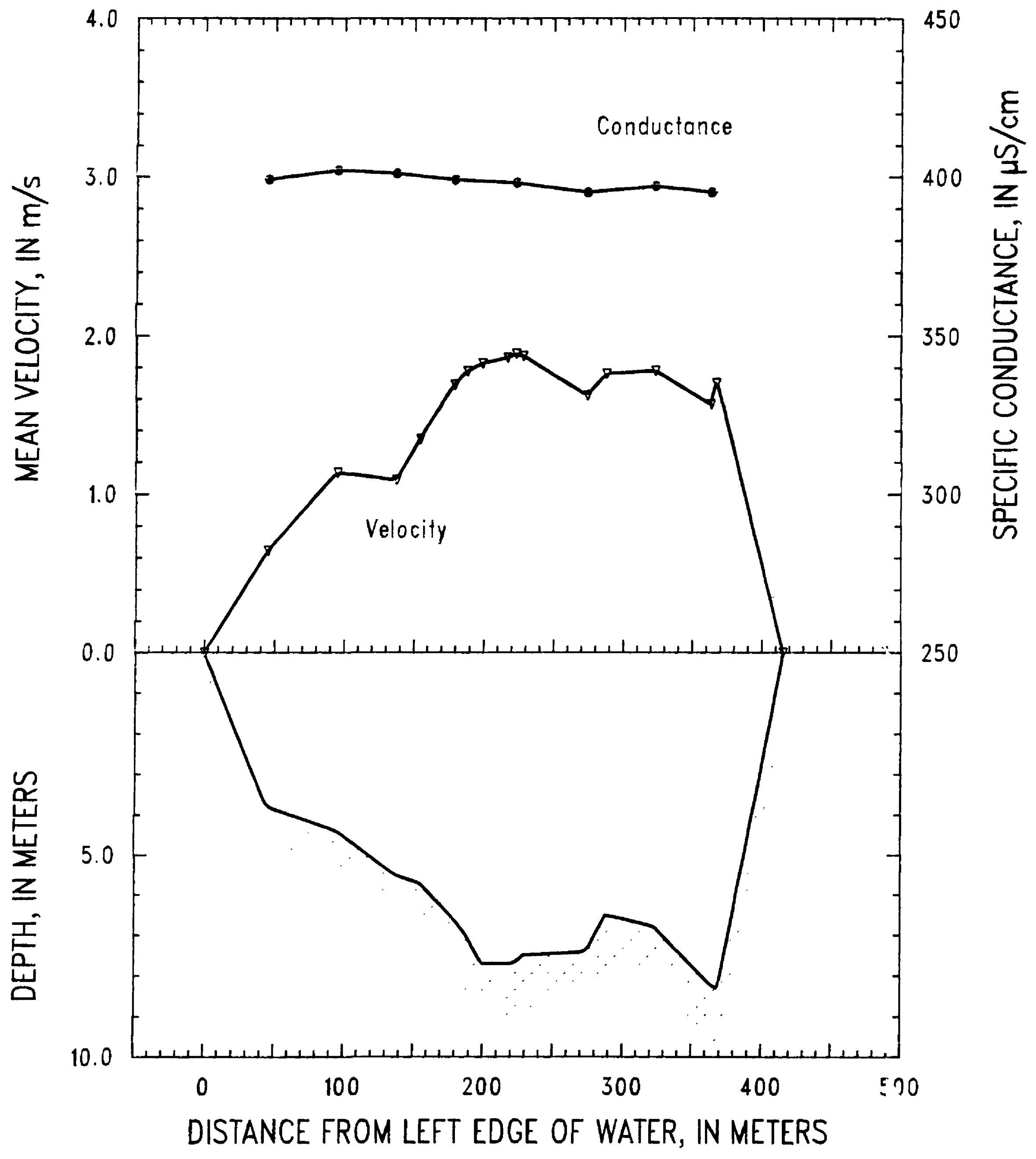

Figure 53. Missouri River at St. Charles, Missouri, on April 29, 1992. 
SITE: Mississippi River at Thebes, Ill.-Mile $\mathbf{4 4 . 0}$

05-01-92

PARTY: Moody, Krest, and Simoneaux

GAGE HEIGHT @ Chester: $364.95 \mathrm{ft}$ GAGE HEIGHT @ Cairo: $305.37 \mathrm{ft}$

RIVER SLOPE: $102.6 \times 10^{-6}$

SUSP. Bag sampler and 200 -lb weight

PRICE AA CURRENT METER No: W-223906 DATE RATED: 06-91

CURRENT METER EQUATION: $V(\mathrm{~m} / \mathrm{s})=\operatorname{Rev} / \mathrm{s}^{*} 0.670+0.010$

REMARKS:

Transit rate was $15 \mathrm{~cm} / \mathrm{s}$ and the nozzle was $3 / 16$ inch. The unmeasured zone was $0.66 \mathrm{~m}$. C rder of verticals was $1,2,3,9,8,7,6,5$, and 4 . Specific conductance of vertical 9 was measured last.

\begin{tabular}{|c|c|c|c|c|c|c|c|}
\hline Vertical & $\begin{array}{l}\text { Distance } \\
\text { from } \\
\text { LEW } \\
\text { (m) }\end{array}$ & $\begin{array}{l}\text { Depth } \\
\text { (m) }\end{array}$ & $\begin{array}{l}\text { Mean } \\
\text { velocity } \\
(\mathrm{m} / \mathrm{s})\end{array}$ & $\begin{array}{c}\text { Discharge } \\
\left(\mathrm{m}^{3} / \mathrm{s}\right)\end{array}$ & $\begin{array}{c}\text { Temperature } \\
\left({ }^{\circ} \mathrm{C}\right)\end{array}$ & pH & $\begin{array}{c}\text { Specific } \\
\text { conductance } \\
(\mu \mathrm{S} / \mathrm{cm})\end{array}$ \\
\hline LEW & 0 & 0.0 & 0.00 & & & & \\
\hline$X_{07}$ & 59 & 10.2 & 0.93 & 333 & $\therefore$ & -- & -- \\
\hline 01A,B & 70 & 10.9 & 0.92 & 292 & 13.4 & 8.3 & 464 \\
\hline 02A,B & 117 & 12.1 & 1.58 & 611 & 14.3 & 8.1 & 467 \\
\hline$X 06$ & 134 & 13.3 & 1.67 & 811 & -. & -- & -- \\
\hline 03A,B & 190 & 12.6 & 1.75 & 1,171 & 13.7 & 8.1 & 465 \\
\hline 04A,B & 240 & 12.1 & 2.02 & 1,492 & 14.4 & 8.1 & 462 \\
\hline $05 \mathrm{~A}, \mathrm{~B}$ & 312 & 12.7 & 2.25 & 1,285 & 14.5 & 8.0 & 462 \\
\hline $\mathrm{X04}$ & 330 & 13.4 & 2.29 & 751 & -- & -- & - \\
\hline $06 \mathrm{~B}$ & 361 & 13.2 & 2.23 & 515 & 14.2 & 8.0 & 460 \\
\hline $\mathrm{X} 10$ & 365 & 12.7 & 2.11 & 134 & .. & - & -- \\
\hline $06 \mathrm{~A}$ & 371 & 12.7 & 2.22 & 1,001 & - & - & -- \\
\hline 07A,B & 436 & 10.5 & 1.70 & 1,019 & 14.0 & 8.0 & 455 \\
\hline $08 \mathrm{~A}$ & 485 & 9.7 & 1.06 & 278 & 14.7 & 8.0 & 455 \\
\hline 08B & 490 & 9.4 & 1.24 & 326 & - & - & -- \\
\hline X01 & 541 & 8.7 & 0.83 & 213 & - & -. & -- \\
\hline $\mathrm{X} 02$ & 549 & 8.3 & 0.77 & 54 & -- & - & - \\
\hline 09A,B & 558 & 8.1 & 0.68 & 187 & 14.7 & 8.0 & 455 \\
\hline REW & 617 & 0.0 & 0.00 & & & & \\
\hline MEANS & & 10.2 & 1.66 & & & & \\
\hline TOTAL & 617 & & & 10,477 & & & \\
\hline
\end{tabular}




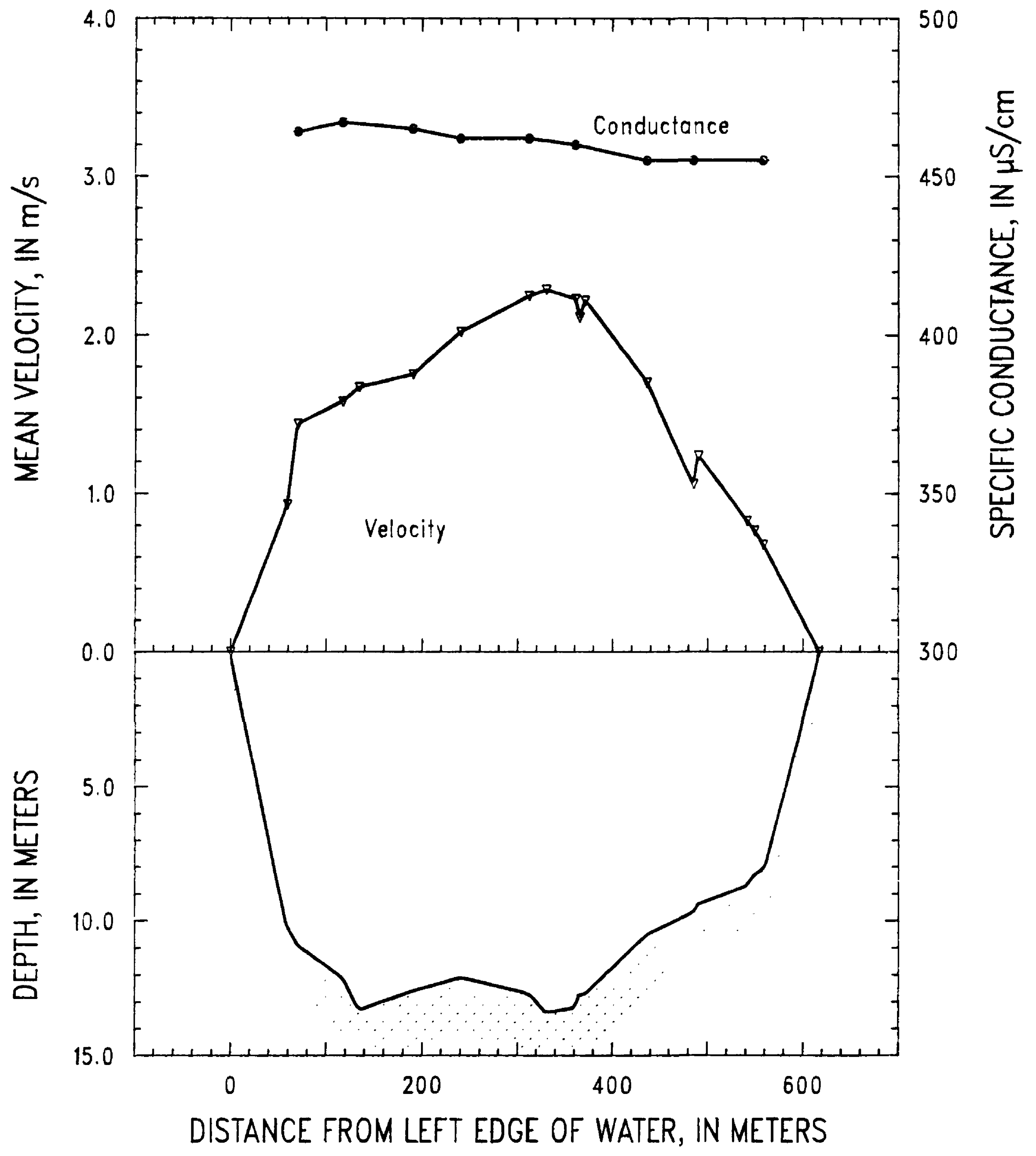

Figure 54. Mississippi River at Thebes, Illinois, on May 1, 1992. 
SITE: Ohio River at Olmsted, Ill.-Mile 965.0

05-03-92

PARTY: Moody, Krest, and Simoneaux

GAGE HEIGHT @ Dam 53: $302.60 \mathrm{ft}$ GAGE HEIGHT @ Cairo: $302.27 \mathrm{ft}$

RIVER SLOPE: $4.3 \times 10^{-6}$

SUSP. Bag sampler and 150-lb weight

PRICE AA CURRENT METER No: W-223906 DATE RATED: 06-91

CURRENT METER EQUATION: V(m/s)=Rev $/ \mathrm{s}^{*} 0.670+0.010$

REMARKS:

Did not collected a depth-integrated composite sample. Transit rate was $5 \mathrm{~cm} / \mathrm{s}$. Unmeasured zone was $0.66 \mathrm{~m}$. Order of verticals was 11-1.

\begin{tabular}{|c|c|c|c|c|c|c|c|}
\hline Vertical & $\begin{array}{c}\text { Distance } \\
\text { from } \\
\text { LEW } \\
\text { (m) }\end{array}$ & $\begin{array}{l}\text { Depth } \\
\text { (m) }\end{array}$ & $\begin{array}{c}\text { Mean } \\
\text { velocity } \\
(\mathrm{m} / \mathrm{s})\end{array}$ & $\begin{array}{c}\text { Discharge } \\
\left(\mathrm{m}^{3} / \mathrm{s}\right)\end{array}$ & $\begin{array}{c}\text { Temperature } \\
\left({ }^{\circ} \mathrm{C}\right)\end{array}$ & pH & $\begin{array}{c}\text { Specific } \\
\text { conductance } \\
(\mu \mathrm{S} / \mathrm{cm})\end{array}$ \\
\hline LEW & 0 & 0.0 & 0.00 & & & & \\
\hline 01 & 91 & 6.9 & 0.52 & 173 & 17.8 & 8.0 & 344 \\
\hline$X 01$ & 97 & 7.2 & 0.47 & 128 & -- & - & - \\
\hline 02 & 167 & 7.4 & 0.49 & 297 & 17.6 & 8.0 & 346 \\
\hline 03 & 261 & 8.8 & 0.55 & 395 & 17.5 & 8.0 & 353 \\
\hline 04 & 331 & 9.9 & 0.56 & 375 & 17.4 & 8.0 & 356 \\
\hline$\times 02$ & 397 & 11.3 & 0.60 & 277 & -- & -- & - \\
\hline 05 & 413 & 10.8 & 0.61 & 351 & 17.4 & 8.0 & 358 \\
\hline 06 & 504 & 12.4 & 0.62 & 636 & 17.5 & 8.0 & 366 \\
\hline 07 & 578 & 12.7 & 0.68 & 372 & 17.4 & 8.0 & 374 \\
\hline$X 03$ & 590 & 12.7 & 0.65 & 362 & -- & -- & -- \\
\hline 08 & 666 & 12.5 & 0.71 & 675 & 17.1 & 8.0 & 389 \\
\hline X04 & 743 & 11.3 & 0.79 & 405 & -. & -- & -- \\
\hline 09 & 757 & 11.7 & 0.79 & 446 & 16.9 & 8.0 & 404 \\
\hline 10 & 840 & 12.3 & 0.71 & 705 & 17.0 & 7.9 & 412 \\
\hline 11 & 919 & 13.2 & 0.49 & 270 & 16.9 & 7.8 & 412 \\
\hline $\mathrm{X} 05$ & 923 & 13.3 & 0.46 & 285 & - & -- & -- \\
\hline REW & 1,012 & 0.0 & 0.00 & & & & \\
\hline MEANS & & 9.9 & 0.62 & & & & \\
\hline TOTAL & 1,012 & & & 6,151 & & & \\
\hline
\end{tabular}




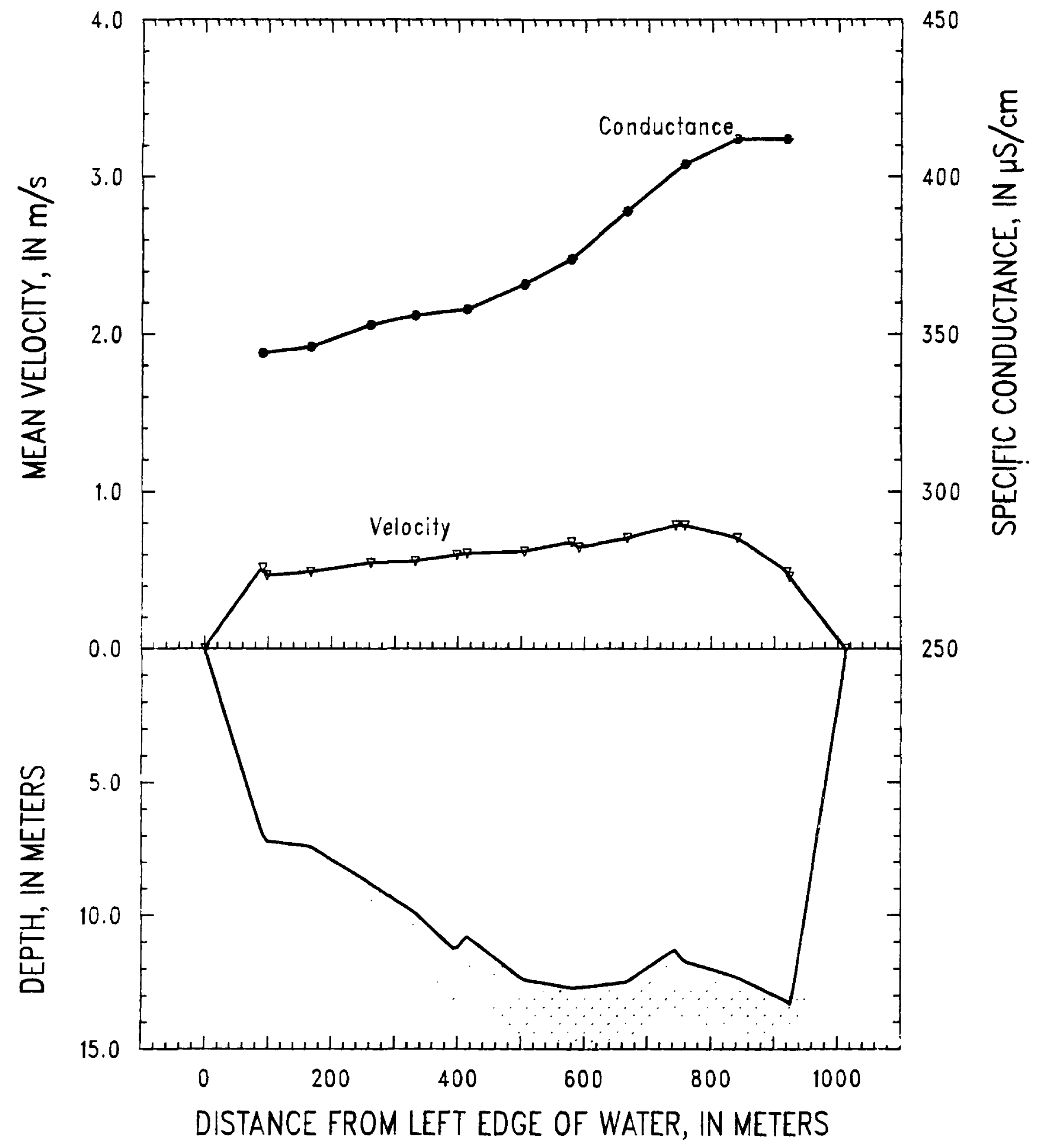

Figure 55. Ohio River at Olmsted, Illinois, on May 3, 1992. 
SITE: Mississippi River below Vicksburg, Miss.-Mile 433.4

$05-06-92$

PARTY: Moody, Krest, and LeBoeuf

GAGE HEIGHT @ Greenville: $109.02 \mathrm{ft}$ GAGE HEIGHT @ Natchez: $52.48 \mathrm{ft}$

RIVER SLOPE: $63.7 \times 10^{-6}$

SUSP. Bag sampler and 200-lb weight

PRICE AA CURRENT METER No: W-223906 DATE RATED: 06-91

CURRENT METER EQUATION: V(m/s)=Rev $/ \mathrm{s}^{*} 0.670+0.010$

REMARKS:

Transit rate was $9.0 \mathrm{~cm} / \mathrm{s}$ and the nozzle was $1 / 8$ inch. Unmeasured zone was $0.66 \mathrm{~m}$. Gage heights and slopes are for May 5, 1992.

\begin{tabular}{|c|c|c|c|c|c|c|c|}
\hline Vertical & $\begin{array}{c}\text { Dlstance } \\
\text { from } \\
\text { LEW } \\
\text { (m) }\end{array}$ & $\begin{array}{l}\text { Depth } \\
\text { (m) }\end{array}$ & $\begin{array}{c}\text { Mean } \\
\text { veiocity } \\
(\mathrm{m} / \mathrm{a})\end{array}$ & $\begin{array}{c}\text { Discharge } \\
\left(\mathrm{m}^{3} / \mathrm{s}\right)\end{array}$ & $\begin{array}{c}\text { Temperature } \\
\left({ }^{\circ} \mathrm{C}\right)\end{array}$ & pH & $\begin{array}{c}\text { Specific } \\
\text { conductance } \\
(\mu \mathrm{S} / \mathrm{cm})\end{array}$ \\
\hline LEW & 0 & 0.0 & 0.00 & & & & \\
\hline$X 06$ & 47 & 8.5 & 0.52 & 116 & -- & -- & -. \\
\hline $01 \mathrm{~A}, \mathrm{~B}$ & 53 & 9.2 & 0.72 & 234 & 17.3 & 8.0 & 385 \\
\hline$X 07$ & 118 & 18.8 & 1.14 & 855 & $\therefore$ & -- & - \\
\hline$\times 05$ & 133 & 19.5 & 1.41 & 288 & - & -- & - \\
\hline $02 \mathrm{~A}, \mathrm{~B}$ & 139 & 19.3 & 1.33 & 720 & 17.1 & 8.0 & 387 \\
\hline 03A,B & 189 & 18.3 & 1.50 & 1,390 & 17.3 & 8.0 & 388 \\
\hline 04A,B & 240 & 18.0 & 1.52 & 1,721 & 17.2 & 8.0 & 389 \\
\hline 05A,B & 315 & 18.1 & 1.50 & 1,388 & 17.1 & 8.0 & 392 \\
\hline X04 & 342 & 18.0 & 1.69 & 865 & - & -- & -- \\
\hline O6A,B & 372 & 18.0 & 1.55 & 1,477 & 17.3 & 8.0 & 393 \\
\hline 07A,B & 448 & 16.7 & 1.55 & 1,818 & 17.5 & 8.0 & 393 \\
\hline 08A,B & 512 & 16.6 & 1.62 & 1,602 & 17.3 & 8.0 & 395 \\
\hline 09A,B & 567 & 16.3 & 1.58 & 903 & 17.3 & 8.0 & 395 \\
\hline$\times 09$ & 582 & 16.8 & 1.57 & 621 & -- & -- & -- \\
\hline$x_{03}$ & 614 & 15.2 & 1.64 & 784 & - & - & -- \\
\hline $10 A, B$ & 645 & 15.3 & 1.53 & 1,100 & 17.5 & 8.0 & 396 \\
\hline $11 \mathrm{~A}, \mathrm{~B}$ & 708 & 14.1 & 1.54 & 1,215 & 17.4 & 8.0 & 396 \\
\hline$\times 10$ & 757 & 13.3 & 1.37 & 510 & -- & - & -. \\
\hline $12 \mathrm{~A}, \mathrm{~B}$ & 764 & 13.5 & 1.22 & 585 & 17.3 & 8.0 & 395 \\
\hline $13 \mathrm{~A}, \mathrm{~B}$ & 828 & 12.0 & 1.20 & 847 & 17.5 & 8.0 & 396 \\
\hline $14 \mathrm{~A}, \mathrm{~B}$ & 882 & 10.9 & 1.13 & 393 & 17.3 & 8.0 & 396 \\
\hline $\mathrm{X02}$ & 892 & 10.5 & 1.19 & 350 & -- & - & -- \\
\hline $15 \mathrm{~A}, \mathrm{~B}$ & 938 & 9.7 & 1.03 & 577 & 17.2 & 8.0 & 396 \\
\hline $16 \mathrm{~A}, \mathrm{~B}$ & 1,008 & 7.8 & 1.11 & 591 & 17.4 & 8.0 & 396 \\
\hline $17 \mathrm{~A}, \mathrm{~B}$ & 1,075 & 6.5 & 0.99 & 436 & 17.1 & 8.0 & 396 \\
\hline X01 & 1,144 & 6.6 & 0.77 & 189 & -- & -- & -- \\
\hline $18 \mathrm{~A}, \mathrm{~B}$ & 1,149 & 6.5 & 0.76 & 170 & 17.3 & 8.0 & 396 \\
\hline REW & 1,213 & 0.0 & 0.00 & & & & \\
\hline MEANS & & 13.0 & 1.37 & & & & \\
\hline TOTAL & 1,213 & & & 21,745 & & & \\
\hline
\end{tabular}




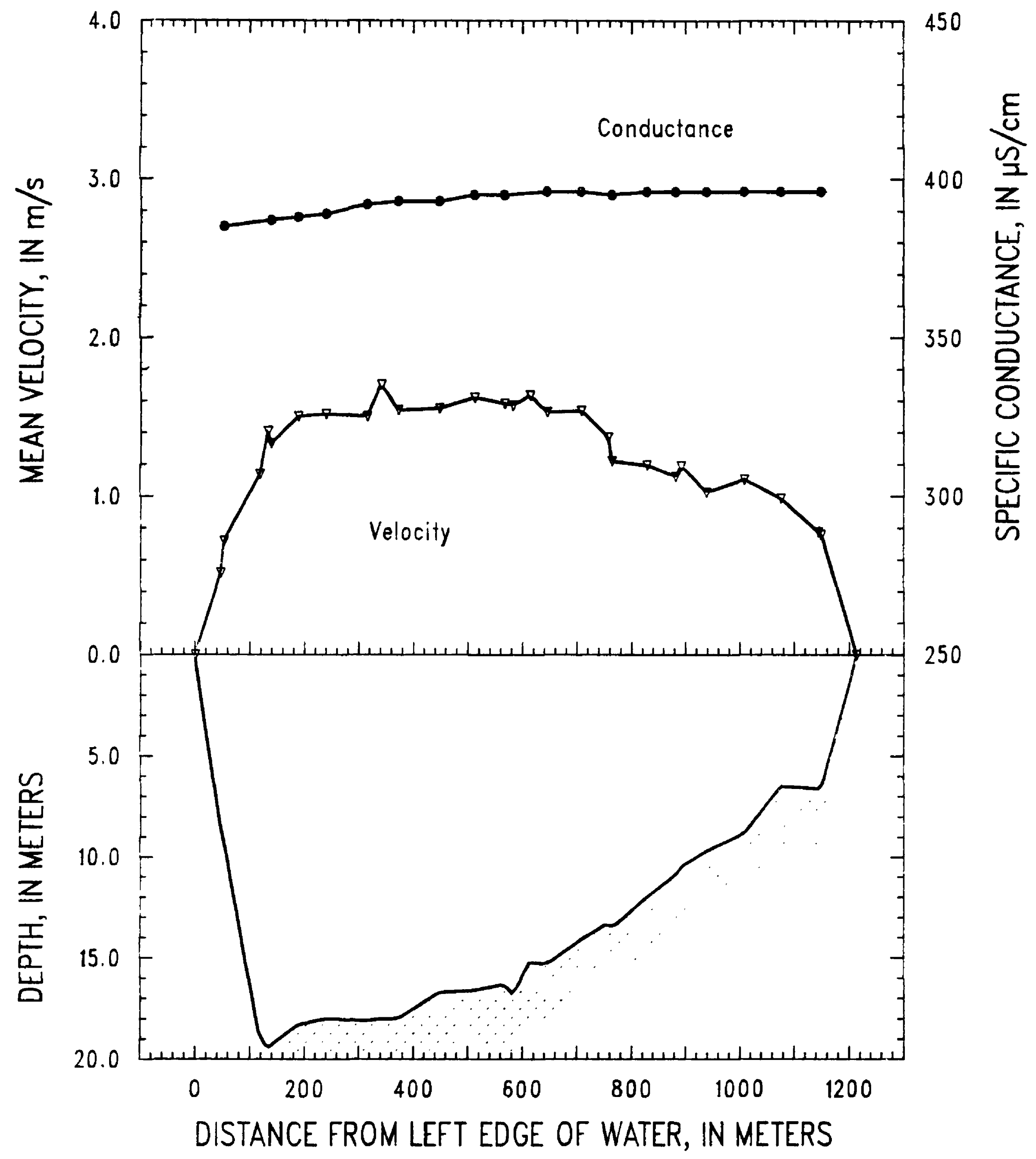

Figure 56. Mississippi River below Vicksburg, Mississippi, on May 6, 1992. 
SITE: Mississippi River near St. Francisville, La.-Mile 266.4

05-08-92

PARTY: Moody, Krest, and LeBoeuf

GAGE HEIGHT @ Red River Landing: $37.3 \mathrm{ft}$ GAGE HEIGHT @ Baton Rouge: $23.8 \mathrm{ft}$

RIVER SLOPE: $34.6 \times 10^{-6}$

SUSP. Bag sampler and 200-lb weight

PRICE AA CURRENT METER No: 90JM1 DATE RATED: 06-91

CURRENT METER EQUATION: $V(\mathrm{~m} / \mathrm{s})=\operatorname{Rev} / \mathrm{s}^{*} 0.667+0.006$

REMARKS:

Transit rate was $13.0 \mathrm{~cm} / \mathrm{s}$ and the nozzle was $3 / 16 \mathrm{inch}$. Unmeasured zone was $0.66 \mathrm{~m}$. No clouds, no wind, mild temperatures-the best day so far of Cruise 92-1.

\begin{tabular}{|c|c|c|c|c|c|c|c|}
\hline Verticai & $\begin{array}{l}\text { Distance } \\
\text { from } \\
\text { LEW } \\
\text { (m) }\end{array}$ & $\begin{array}{l}\text { Depth } \\
\text { (m) }\end{array}$ & $\begin{array}{c}\text { Mean } \\
\text { velocity } \\
(\mathbf{m} / \mathbf{s})\end{array}$ & $\begin{array}{c}\text { Discharge } \\
\left(\mathrm{m}^{3} / \mathrm{s}\right)\end{array}$ & $\begin{array}{c}\text { Temperature } \\
\left({ }^{\circ} \mathrm{C}\right)\end{array}$ & pH & $\begin{array}{c}\text { Specific } \\
\text { conductance } \\
(\mu \mathrm{S} / \mathrm{cm})\end{array}$ \\
\hline LEW & 0 & 0.0 & & & & & \\
\hline $01 \mathrm{~A}, \mathrm{~B}$ & 65 & 8.2 & 0.83 & 246 & 17.6 & 8.0 & 390 \\
\hline X06 & 72 & 8.7 & 0.80 & 205 & - & -.. & - \\
\hline $02 \mathrm{~A}, \mathrm{~B}$ & 124 & 11.2 & 0.78 & 470 & 17.6 & 8.0 & 391 \\
\hline 03A,B & 180 & 11.5 & 1.01 & 484 & 17.7 & 7.9 & 389 \\
\hline X05 & 207 & 10.9 & 1.05 & 400 & -.. & -.. & -- \\
\hline 04A,B & 250 & 10.7 & 1.21 & 577 & 17.9 & 7.8 & 391 \\
\hline 05A,B & 296 & 11.4 & 1.27 & 794 & 17.8 & 7.9 & 390 \\
\hline 06A,B & 360 & 11.3 & 1.34 & 1019 & 17.8 & 7.9 & 390 \\
\hline 07A,B & 431 & 11.7 & 1.42 & 963 & 18.1 & 7.9 & 390 \\
\hline 08A,B & 476 & 11.4 & 1.35 & 874 & 17.8 & 7.9 & 390 \\
\hline $09 \mathrm{~A}, \mathrm{~B}$ & 545 & 12.8 & 1.43 & 1125 & 17.9 & 7.9 & 389 \\
\hline X03 & 599 & 13.3 & 1.26 & 493 & -- & -- & -- \\
\hline $10 \mathrm{~A}, \mathrm{~B}$ & 604 & 13.3 & 1.20 & 568 & 17.7 & 7.9 & 392 \\
\hline $11 \mathrm{~A}, \mathrm{~B}$ & 670 & 13.9 & 1.26 & 1013 & 17.7 & 7.9 & 389 \\
\hline $12 \mathrm{~A}, \mathrm{~B}$ & 720 & 16.3 & 1.14 & 1062 & 18.0 & 7.9 & 389 \\
\hline $13 \mathrm{~A}, \mathrm{~B}$ & 784 & 18.3 & 1.15 & 1325 & 18.5 & 7.9 & 389 \\
\hline $14 \mathrm{~A}, \mathrm{~B}$ & 846 & 18.1 & 1.23 & 823 & 18.2 & 7.9 & 390 \\
\hline X02 & 858 & 18.0 & 1.29 & 606 & --- & -- & -- \\
\hline $15 \mathrm{~A}, \mathrm{~B}$ & 898 & 19.3 & 1.14 & 1089 & 17.7 & 7.9 & 390 \\
\hline $16 \mathrm{~A}, \mathrm{~B}$ & 957 & 15.5 & 1.10 & 993 & 17.5 & 7.9 & 392 \\
\hline REW & 1,015 & 0.0 & & & & & \\
\hline MEANS & & 12.6 & 1.18 & & & & \\
\hline TOTAL & 1,015 & & & 15,128 & & & \\
\hline
\end{tabular}




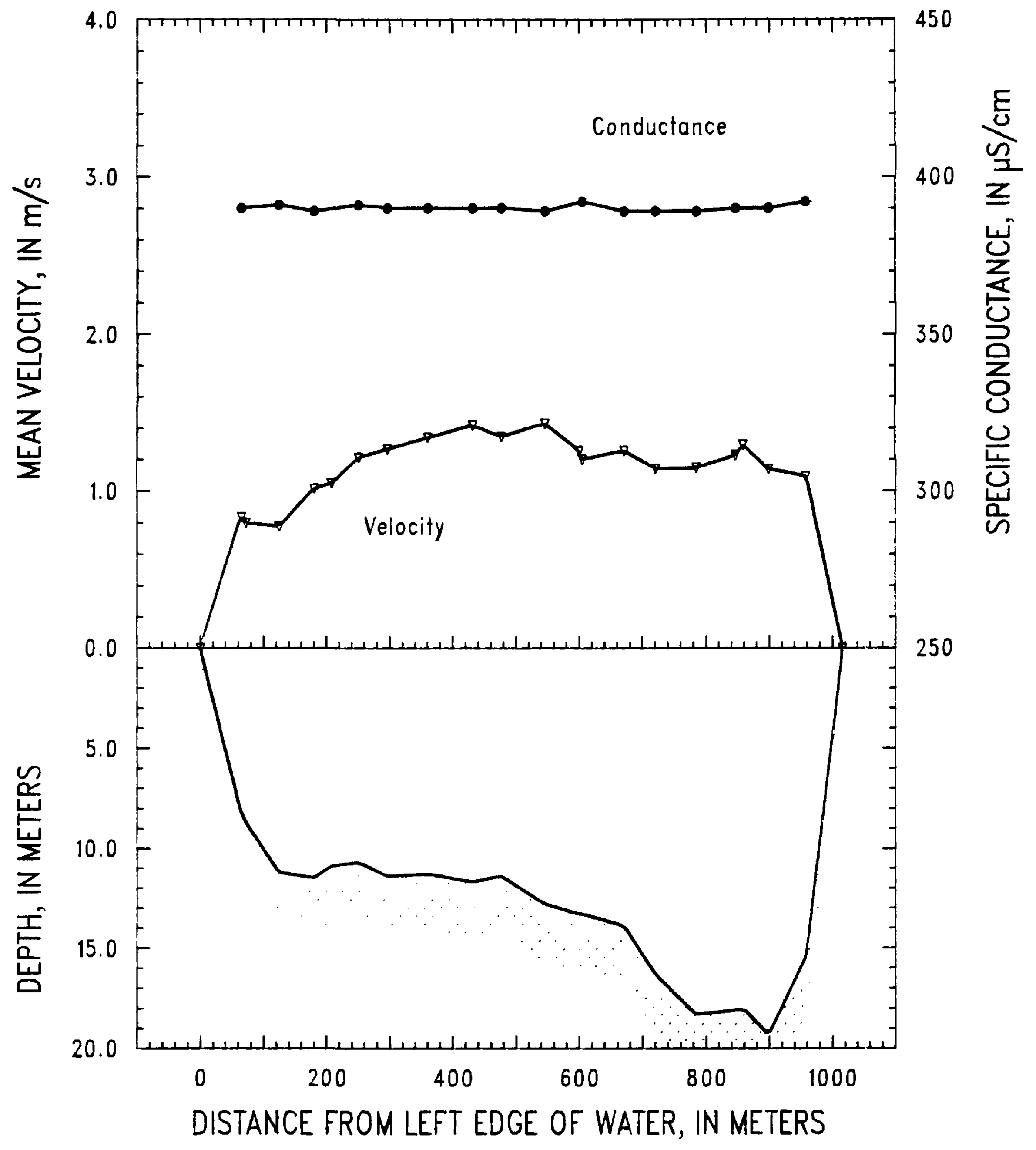

Figure 57. Mississippi River near St. Francisville, Louisiana, on May 8, 1992. 
SITE: Mississippi River below Belle Chasse, La.-Mile 73.1

PARTY: Moody, Krest, and LeBoeuf

GAGE HEIGHT @ Baton Rouge: $23.8 \mathrm{ft} \quad$ GAGE HEIGHT @ New Orleans: $7.8 \mathrm{ft}$

RIVER SLOPE: $24.1 \times 10^{-6}$

SUSP. Bag sampler and 200-lb weight

PRICE AA CURRENT METER No: 90JM1 DATE RATED: 06-91

CURRENT METER EQUATION: $V(\mathrm{~m} / \mathrm{s})=\operatorname{Rev} / \mathrm{s}^{*} 0.667+0.006$

REMARKS:

Transit rate was $13.0 \mathrm{~cm} / \mathrm{s}$ and the nozzle was $3 / 16 \mathrm{inch}$. Unmeasured zone was $0.66 \mathrm{~m}$. Gage heights and slope are for May 8, 1992. Order of verticals was 7-1.

\begin{tabular}{|c|c|c|c|c|c|c|c|}
\hline Vertical & $\begin{array}{l}\text { Distance } \\
\text { from } \\
\text { LEW } \\
\text { (m) }\end{array}$ & $\begin{array}{l}\text { Depth } \\
\text { (m) }\end{array}$ & $\begin{array}{l}\text { Mean } \\
\text { veloclty } \\
(\mathrm{m} / \mathrm{s})\end{array}$ & $\begin{array}{c}\text { Discharge } \\
\left(\mathrm{m}^{3} / \mathrm{s}\right)\end{array}$ & $\begin{array}{c}\text { Temperature } \\
\left({ }^{\circ} \mathrm{C}\right)\end{array}$ & pH & $\begin{array}{c}\text { Specific } \\
\text { conductance } \\
(\mu \mathrm{S} / \mathrm{cm})\end{array}$ \\
\hline LEW & 0 & 0.0 & 0.00 & & & & \\
\hline X05 & 48 & 11.2 & 0.65 & 358 & - & - & - \\
\hline 01B & 98 & 18.0 & 0.80 & 380 & - & - & - \\
\hline $01 \mathrm{~A}$ & 101 & 19.3 & 0.84 & 878 & 18.7 & 7.8 & 402 \\
\hline $02 \mathrm{~A}, \mathrm{~B}$ & 207 & 21.3 & 1.06 & 2,299 & 18.6 & 7.7 & 402 \\
\hline 03A,B & 305 & 24.3 & 1.04 & 2,379 & 18.8 & 7.8 & 403 \\
\hline $\mathrm{X} 03$ & 396 & 25.3 & 0.95 & 1,228 & -- & - & - \\
\hline 04A,B & 407 & 25.4 & 0.93 & 1,287 & 18.7 & 7.8 & 402 \\
\hline $05 \mathrm{~A}, \mathrm{~B}$ & 505 & 25.5 & 0.89 & 2,171 & 18.5 & 7.8 & 400 \\
\hline X02 & 599 & 24.7 & 0.88 & 1,092 & - & - & -- \\
\hline 06A,B & 606 & 24.2 & 0.81 & 1,027 & 18.5 & 7.8 & 400 \\
\hline 07A,B & 704 & 22.4 & 0.71 & 1,053 & 18.4 & 7.8 & 399 \\
\hline X01 & 738 & 15.0 & 0.57 & 391 & - & - & -- \\
\hline REW & 795 & 0.0 & 0.00 & & & & \\
\hline MEANS & & 20.6 & 0.89 & & & & \\
\hline TOTAL & 795 & & & 14,542 & & & \\
\hline
\end{tabular}




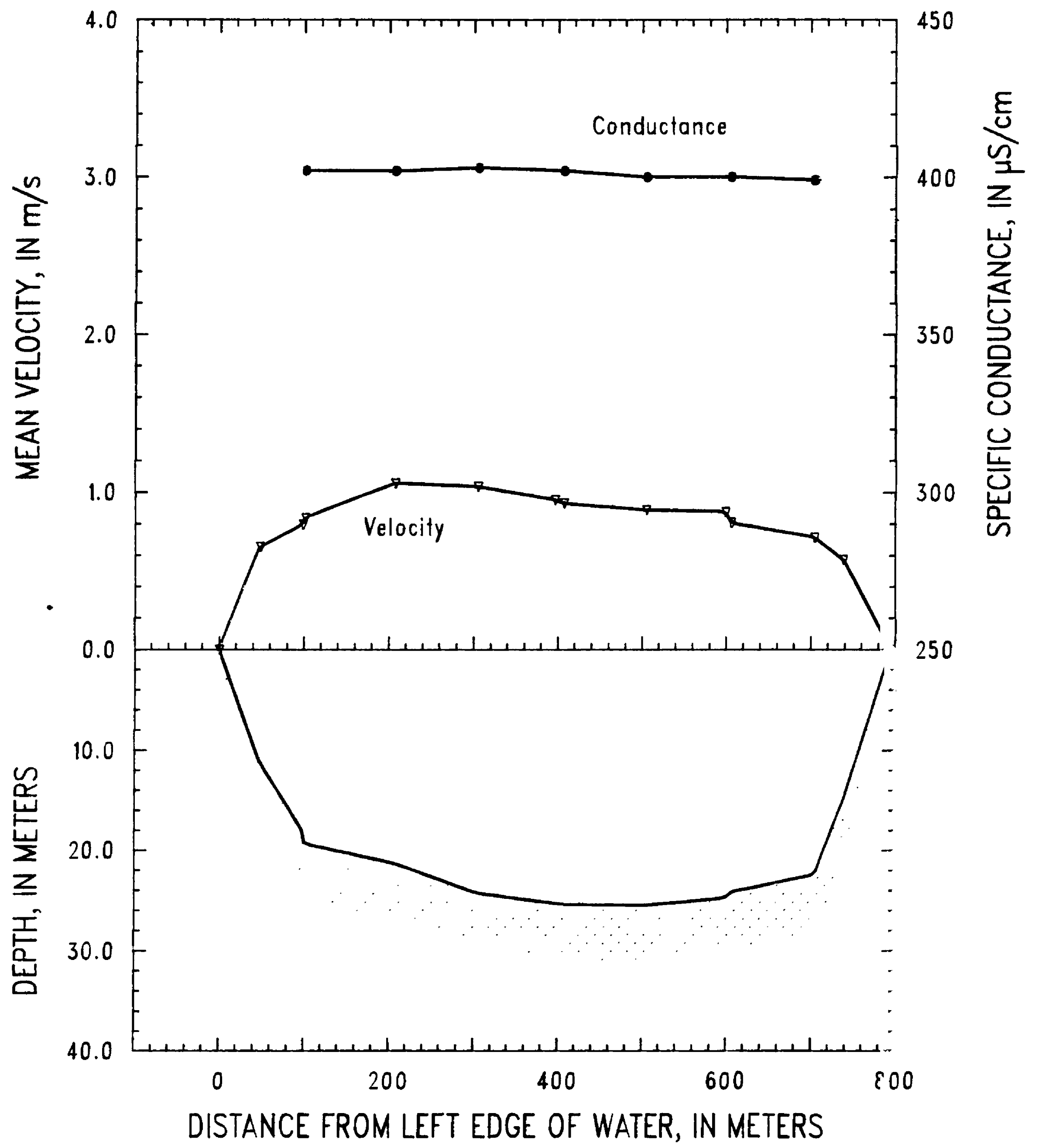

Figure 58. Mississippi River below Belle Chasse, Louisiana, on May 10, 1992. 